\title{
Generalizações do teorema de representação de Riesz
}

\author{
DISSERTAÇÃO APRESENTADA \\ $\mathrm{AO}$ \\ INSTITUTO DE MATEMÁTICA E ESTATÍSTICA \\ DA \\ UNIVERSIDADE DE SÃO PAULO \\ PARA \\ OBTENÇÃO DO TÍTULO \\ $\mathrm{DE}$ \\ MESTRE EM CIÊNCIAS \\ Programa: Matemática \\ Orientador: Prof. Dr. Daniel Victor Tausk
}

Durante o desenvolvimento deste trabalho o autor recebeu auxílio financeiro do $\mathrm{CNPq}$

São Paulo, junho de 2009 



\section{Generalizações do teorema de representação de Riesz}

Este exemplar corresponde à redação final da dissertação devidamente corrigida e defendida por Cesar Adriano Batista e aprovada pela Comissão Julgadora.

Banca examinadora:

- Prof. Dr. Daniel Victor Tausk

IME - USP

- Prof. Dr. Luiz Fichmann

IME - USP

- Prof. Dr. Pedro Luis Aparecido Malagutti

UFSCar 

À minha mãe, Amélia, e ao meu pai, Jõ̃o. 



\section{Agradecimentos}

Agradeço a todos que de algum modo contribuíram para a concretização deste trabalho, em especial:

Aos meus amigos Bárbara, Carol, Gustavo, Natália, Rodrigo, Sílvia e Zé, entre tantas coisas, pelo apoio, amizade, inspiração e presença em momentos felizes e também em outros não tão felizes.

Aos meus colegas do IME Armando, Danilo, Dilene, Flausino, Héctor, Josnei, Lorena, Mariana, Nazar, Núbia, Oscar, Pedro, Priscila, Renato, Rodrigo Ferraz, Rodrigo Freire, Samir, Sandra, Sávio e Tatiane, entre outros, pelo convívio, pelas interações acadêmicas, pelas batalhas compartilhadas e pelo respeito constante.

Aos professores Alfredo Aragona, Cesar Polcino, Daniela Mariz, Elói Medina, Iole Druck, Juan Carlos Fernandez, Leila Figueiredo, Lúcia Junqueira, Luiz Peresi, Marcos Alexandrino, Cristina Barufi, Rosa Maria, Roseli Fernandez, Vera Carrara e Zara Abud, pela cooperação direta ou indireta na realização deste projeto.

Pela forma sempre atenciosa com que fui atendido, e por seu fundamental trabalho de "bastidores", aos funcionários Alessandra, Emerson, Marilucia e Pinho, da secretaria de pós-graduação, Francisca e Rose, da secretária de graduação, Carlos, Cláudio, Creusa, Max e Rafael, da gráfica, dona Jovita e dona Dalvina, da Copa, Francisca e Paulo, dos serviços gerais, Gislaine, dona Jeane e Xavier, do CEC, Feijão e Sérgio, da seção de informática, Beth e Célia, da biblioteca.

Ao $\mathrm{CNPq}$ pelo financiamento parcial deste trabalho.

Aos professores Luiz Fichmann e Pedro Malagutti por gentilmente aceitarem compor a banca e pelas valiosas sugestões que ajudaram a melhorar esta dissertação.

Ao meu orientador, professor Daniel Victor Tausk, por sua disponibilidade e paciência infinitas, pelas inúmeras sugestões sem as quais este trabalho certamente perderia em qualidade, e principalmente por ter aceitado novamente a tarefa nada fácil de orientar este "filho pródigo".

À minha família, pela torcida e pelo amor e ajuda incondicionais, sobretudo aos meus pais, sem os quais nada disso seria possível. 
À minha namorada, Raíssa, por seu amor, carinho, amizade, dedicação, compreensão e por estar ao meu lado em todos os momentos, inclusive naqueles mais difíceis e quase insuportáveis.

Por fim, agradeço novamente a meu pai, que durante toda sua vida e até o fim dela, trabalhou duro e incansavelmente por sua família. A ele, onde quer que esteja, dedico de modo especial o meu trabalho, e agradeço por todas as coisas que fez por mim nessa vida e por todos os ensinamentos que sobrevivem silenciosamente em mim. 


\section{Resumo}

Dados um espaço de medida $(X, \mathcal{A}, \mu)$ e números reais $p, q \in] 1,+\infty[$ com $\frac{1}{p}+\frac{1}{q}=1$, o Teorema de Representação de Riesz afirma que $L^{q}(X, \mathcal{A}, \mu)$ é o dual topológico de $L^{p}(X, \mathcal{A}, \mu)$ e que $L^{\infty}(X, \mathcal{A}, \mu)$ é o dual topológico de $L^{1}(X, \mathcal{A}, \mu)$ se o espaço $(X, \mathcal{A}, \mu)$ for $\sigma$-finito. Observamos que a $\sigma$-finitude de $(X, \mathcal{A}, \mu)$ é condição suficiente mas não necessária para que $L^{\infty}(X, \mathcal{A}, \mu)$ seja o dual de $L^{1}(X, \mathcal{A}, \mu)$. Os contra-exemplos tipicamente apresentados para $L^{\infty}(X, \mathcal{A}, \mu) \cong L^{1}(X, \mathcal{A}, \mu)^{*}$ são "triviais", no sentido de que desaparecem se "consertarmos" a medida $\mu$, transformando-a numa medida perfeita. Neste trabalho apresentamos condições suficientes mais fracas que $\sigma$-finitude a fim de que $L^{\infty}(X, \mathcal{A}, \mu)$ e $L^{1}(X, \mathcal{A}, \mu)^{*}$ possam ser isometricamente identificados. Além disso, introduzimos um invariante cardinal para espaços de medida que chamaremos a dimensão do espaço e mostramos que se o espaço $(X, \mathcal{A}, \mu)$ for de medida perfeita e tiver dimensão menor ou igual à cardinalidade do continuum então uma condição necessária e suficiente para $L^{\infty}(X, \mathcal{A}, \mu) \cong L^{1}(X, \mathcal{A}, \mu)^{*}$ é que $X$ admita uma decomposição.

\section{Abstract}

Given a measure space $(X, \mathcal{A}, \mu)$ and real numbers $p, q \in] 1,+\infty[$ with

$\frac{1}{p}+\frac{1}{q}=1$, the Riesz Representation Theorem states that $L^{q}(X, \mathcal{A}, \mu)$ is the topological dual space of $L^{p}(X, \mathcal{A}, \mu)$ and that $L^{\infty}(X, \mathcal{A}, \mu)$ is the topological dual space of $L^{1}(X, \mathcal{A}, \mu)$ if $(X, \mathcal{A}, \mu)$ is $\sigma$-finite. We observe that the $\sigma$-finiteness of $(X, \mathcal{A}, \mu)$ is a sufficient but not necessary condition for $L^{\infty}(X, \mathcal{A}, \mu)$ to be the dual of $L^{1}(X, \mathcal{A}, \mu)$. The counter-examples that are typically presented for $L^{\infty}(X, \mathcal{A}, \mu) \cong L^{1}(X, \mathcal{A}, \mu)^{*}$ are "trivial", in the sense that they vanish if we fix the measure $\mu$, making it into a perfect measure. In this work we present sufficient conditions weaker than $\sigma$-finiteness in order that $L^{\infty}(X, \mathcal{A}, \mu)$ and $L^{1}(X, \mathcal{A}, \mu)^{*}$ can be isometrically identified. Moreover, we introduce a cardinal invariant for measure spaces which we call the dimension of the space and we show that if the space $(X, \mathcal{A}, \mu)$ has perfect measure and dimension less than or equal to the cardinal of the continuum then a necessary and sufficient condition for $L^{\infty}(X, \mathcal{A}, \mu) \cong L^{1}(X, \mathcal{A}, \mu)^{*}$ is that $X$ admits a decomposition. 



\section{Sumário}

Introdução $\quad$ xiii

Capítulo 1. Preliminares 1

1.1. Tópicos de Teoria dos Conjuntos 1

1.2. Um Mínimo de Álgebra Linear e Espaços Métricos 8

1.3. Tópicos de Análise Funcional 11

$\begin{array}{ll}\text { 1.4. Tópicos de Teoria da Medida } & 19\end{array}$

Capítulo 2. Espaços de medida perfeita $\quad 55$

2.1. Medidas livres de blocos $\quad 55$

2.2. Medidas cheias $\quad 68$

2.3. Medidas perfeitas $\quad 76$

2.4. Um contra-exemplo não trivial para a bijetividade da Aplicação $\begin{array}{ll}\text { de Riesz } & 81\end{array}$

$\begin{array}{lll}\text { Capítulo 3. Somas } & 87\end{array}$

$\begin{array}{ll}\text { 3.1. Soma externa } & 87\end{array}$

3.2. Norma e Soma direta de tipo $\mathbf{L}^{\mathbf{p}} \quad 90$

Capítulo 4. Decomposição de um espaço de medida e o Teorema de $\begin{array}{ll}\text { Riesz } & 101\end{array}$

4.1. Decomposições essenciais e decomposições 101

4.2. Generalizando o Teorema de Representação de Riesz 112

$\begin{array}{ll}\text { Referências Bibliográficas } & 125\end{array}$ 



\section{Introdução}

Os principais resultados e exemplos deste trabalho estão baseados em notas não publicadas do Prof. Daniel Victor Tausk, do Instituto de Matemática e Estatística da USP. Em [10], [19], [20], [22], [23] e [25] podemos encontrar, em linguagem completamente diferente, resultados similares àqueles obtidos (de modo independente) pelo professor Tausk.

Seja $(X, \mathcal{A}, \mu)$ um espaço de medida, i.e., $X$ é um conjunto, $\mathcal{A}$ é uma $\sigma$-álgebra de subconjuntos de $X$ e $\mu: \mathcal{A} \rightarrow[0,+\infty]$ é uma medida. Dada uma função mensurável $f: X \rightarrow \mathbb{R}$ e $p \in[1,+\infty$ [ definimos:

$$
\begin{gathered}
\|f\|_{p}=\left(\int_{X}|f|^{p} \mathrm{~d} \mu\right)^{\frac{1}{p}} \in[0,+\infty], \text { e } \\
\|f\|_{\infty}=\inf \{c \in[0,+\infty]:|f| \leq c, \mu \text {-quase sempre }\} \in[0,+\infty] .
\end{gathered}
$$

Denotando por $\mathcal{M}(X, \mathcal{A})$ o espaço vetorial real das funções mensuráveis a valores reais sobre $X$ e por $\overline{\mathcal{M}}(X, \mathcal{A}, \mu)$ o quociente de $\mathcal{M}(X, \mathcal{A})$ pelo subespaço das aplicações que se anulam $\mu$-quase sempre, $L^{p}(X, \mathcal{A}, \mu)$ denotará, como é usual, o subespaço de $\overline{\mathcal{M}}(X, \mathcal{A}, \mu)$ consistindo das classes de aplicações $f$ com $\|f\|_{p}<+\infty$. Por abuso de linguagem (muito comum), diremos que $f$ está em $L^{p}(X, \mathcal{A}, \mu)$ significando que a classe de aplicações iguais a $f \mu$-qs está em $L^{p}(X, \mathcal{A}, \mu)$. O espaço vetorial $L^{p}(X, \mathcal{A}, \mu)$ torna-se uma espaço de Banach quando munido com a norma $\|\cdot\|_{p}$. Dados $\left.p, q \in\right] 1,+\infty[$ com $\frac{1}{p}+\frac{1}{q}=1$ então o bem conhecido Teorema de Representação de Riesz afirma que a $(q, p)$-aplicação de Riesz,

$$
\begin{gathered}
L^{q}(X, \mathcal{A}, \mu) \ni g \longmapsto \alpha_{g} \in L^{p}(X, \mathcal{A}, \mu)^{*}, \\
\alpha_{g}(f)=\int_{X} f g \mathrm{~d} \mu, \quad f \in L^{p}(X, \mathcal{A}, \mu),
\end{gathered}
$$

é uma isometria linear, em que $L^{p}(X, \mathcal{A}, \mu)^{*}$ denota o espaço dual topológico de $L^{p}(X, \mathcal{A}, \mu)$. Se $q=1$ e $p=+\infty$ então a $(q, p)$-aplicação de Riesz é uma imersão isométrica mas não é sobrejetora até mesmo para espaços 
de medida $(X, \mathcal{A}, \mu)$ razoavelmente simples. Se, por outro lado, $q=+\infty$ e $p=1$ então sabemos que a $(q, p)$-aplicação de Riesz é uma isometria linear se $(X, \mathcal{A}, \mu)$ é $\sigma$-finito, i.e., se $X$ pode ser coberto por uma quantidade enumerável de subconjuntos mensuráveis de medida finita. Neste trabalho estamos preocupados em estudar condições gerais sobre o espaço de medida $(X, \mathcal{A}, \mu)$ mediante as quais a aplicação de Riesz,

$$
L^{\infty}(X, \mathcal{A}, \mu) \ni g \longmapsto \alpha_{g} \in L^{1}(X, \mathcal{A}, \mu)^{*}
$$

com $\alpha_{g}$ definido como acima, é uma isometria linear. Observe que se $X$ é um conjunto arbitrário, $\mathcal{A}=\mathcal{P}(X)$ é a $\sigma$-álgebra de todos os subconjuntos de $X$ e $\mu$ é a medida de contagem $(\mu(A)=$ número de elementos de $A)$, então a aplicação de Riesz (1) é uma isometria; entretanto, se $X$ é não enumerável então $(X, \mathcal{A}, \mu)$ não é $\sigma$-finito. Portanto, $\sigma$-finitude do espaço de medida não é condição necessária para a aplicação de Riesz ser uma isometria. Em [21] é apresentada uma condição suficiente mais fraca que $\sigma$-finitude (e satisfeita por medidas de contagem) para a aplicação de Riesz ser uma isometria. A condição é que o espaço $X$ admita uma decomposição; em [21] um decomposição para $X$ é uma partição $X=\bigcup_{i \in I} X_{i}$ de $X$ em subconjuntos mensuráveis $X_{i}$, dois a dois disjuntos, com $\mu\left(X_{i}\right)<+\infty$ para todo $i \in I$, satisfazendo a seguinte propriedade: se $A$ é um subconjunto de $X$ com $A \cap X_{i} \in \mathcal{A}$ e $\mu\left(A \cap X_{i}\right)=0$ para todo $i \in I$ então $A$ é mensurável e $\mu(A)=0$. Por exemplo, se $\mathcal{A}=\mathcal{P}(X)$ e $\mu$ é a medida de contagem então $X=\bigcup_{x \in X}\{x\}$ é uma decomposição para $X$.

É essa condição suficiente mais fraca que $\sigma$-finitude apresentada em [21] o ponto de partida para as investigações apresentadas nesta dissertação. No intuito de compreender melhor os obstáculos para a bijetividade da aplicação de Riesz (1) e oferecer condições suficientes mais fracas (que $\sigma$-finitude) para ela, efetuamos a princípio modificações no espaço de medida $(X, \mathcal{A}, \mu)$ no sentido de obter, a partir de $(X, \mathcal{A}, \mu)$, o que chamaremos de espaço de medida perfeita. É o que fazemos no Capítulo 2. Nesse capítulo definimos as noções de bloco infinito e de medida livre de blocos, e mostramos que a aplicação de Riesz (1) de um espaço de medida $(X, \mathcal{A}, \mu)$ é injetora se somente se a medida $\mu$ é livre de blocos. Afim de tornar a medida do espaço mais "adequada" para a bijetividade de (1), poderíamos pensar que a saída seria livrarmos-nos dos blocos infinitos, "isolando-os"; com o Exemplo 2.1.6 
mostramos que tentar isolar os blocos infinitos não é a melhor estratégia para nos livrarmos deles. Procuramos então consertar a medida $\mu$ do espaço $(X, \mathcal{A}, \mu)$ criando uma versão livre de blocos para ela. Num segundo momento vemos, através do Exemplo 2.2.2, que a $\sigma$-álgebra $\mathcal{A}$ do espaço de medida $(X, \mathcal{A}, \mu)$ pode não ser suficientemente "grande" para tornar mensuráveis as aplicações necessárias para que a aplicação de Riesz (1) seja sobrejetora; construímos o conceito de medida cheia na tentativa de corrigir essa deficiência. Uma medida perfeita será definida como uma medida que é simultaneamente completa, cheia e livre de blocos. Para espaços de medida perfeita são "melhores" as chances da aplicação de Riesz (1) ser uma isometria mas, infelizmente, não necessariamente é esse o caso. Um contraexemplo não trivial (ou seja, que está livre dos obstáculos "ingênuos" para a bijetividade da aplicação de Riesz) é oferecido para evidenciar esse fato. Diante disso, dado um espaço de medida $(X, \mathcal{A}, \mu)$, nossa atenção e esforço volta-se para oferecer uma noção de decomposição (ligeiramente diferente daquele apresentada em [21]), de tal modo que para espaços de medida perfeita que admitam uma decomposição tenhamos que a aplicação de Riesz (1) seja uma isometria.

Veremos no Capítulo 4 que uma família essencialmente disjunta de $(X, \mathcal{A}, \mu)$ é uma família $\left(X_{i}\right)_{i \in I}$ de subconjuntos mensuráveis de $X$ tal que $\mu\left(X_{i} \cap X_{j}\right)=0$ para todo $i, j \in I$ com $i \neq j$. Definimos uma decomposição essencial para $(X, \mathcal{A}, \mu)$ como uma família essencialmente disjunta $\left(X_{i}\right)_{i \in I}$ tal que cada $X_{i}$ tem medida positiva finita, e se $A \subset X$ é um conjunto mensurável de medida finita e $\mu\left(A \cap X_{i}\right)=0$ para todo $i \in I$, então $\mu(A)=0$. Uma decomposição para $(X, \mathcal{A}, \mu)$ será definida como uma decomposição essencial $\left(X_{i}\right)_{i \in I}$ para $X$ na qual os conjuntos $X_{i}$ são dois a dois disjuntos. Mostraremos que todo espaço de medida $(X, \mathcal{A}, \mu)$ admite uma decomposição essencial e que se $\mu_{\mathrm{lb}}(X)=+\infty$ ( $\mu_{\mathrm{lb}}$ a versão livre de blocos da medida $\mu$ ) então duas decomposições essenciais para $X$ têm a mesma cardinalidade. Isso permite-nos estabelecer um invariante cardinal que chamaremos de dimensão do espaço de medida $(X, \mathcal{A}, \mu)$, e mostramos que uma condição suficiente para $(X, \mathcal{A}, \mu)$ admitir uma decomposição é que sua dimensão seja menor ou igual a $\aleph_{1}$. Provamos que para um espaço de medida perfeita, cuja dimensão seja menor ou igual a $\aleph_{1}$, a sua aplicação de 
Riesz (1) é uma isometria linear, e por fim mostramos que para espaços de medida perfeita de dimensão menor ou igual à cardinalidade do continuum, sua aplicação de Riesz (1) é uma isometria linear se e somente se o espaço admite uma decomposição.

No Capítulo 3 estudamos as somas externas e definimos os conceitos de norma de tipo- $L^{p}$ e soma de tipo- $L^{p}$ para uma família de espaços de Banach. Mostramos que para uma família $\left(X_{i}, \mathcal{A}_{i}, \mu_{i}\right)_{i \in I}$ de espaços de medida perfeita a sua soma externa também tem medida perfeita. Evidenciamos e provamos certas isometrias entre somas de tipo- $L^{p}$ e soma externa que serão essenciais para mostrar a comutatividade do diagrama (4.2.3), fundamental para provar os resultados mencionados no parágrafo anterior sobre a aplicação de Riesz.

No Capítulo 1 estabelecemos as principais definições, notações e resultados sobre teoria dos conjuntos, álgebra linear, espaços métricos, análise funcional e teoria da medida que serão utilizados ao longo dos demais capítulos. Não pretendemos, obviamente, esgotar tais assuntos, mas somente destacar algumas noções diretamente relacionadas ao problema a ser estudado. Desse modo, não necessariamente ocorre uma transição "suave" de uma noção para outra. Demonstramos boa parte dos resultados, sobretudo na Seção 1.4 (tópicos de teoria da medida); para outra boa parte deles omite-se intencionalmente sua demonstração sem nenhuma referência específica, como é o caso dos resultados da Seção 1.1 (tópicos de teoria dos conjuntos); e para o restante dos resultados omitimos sua demonstração, mas oferecemos uma referência bibliográfica para ela, como por exemplo o Teorema de Representação de Riesz da Seção 1.4. Todos os espaços vetoriais que figuram neste trabalho são reais, ou seja, $\mathbb{R}$ é o corpo de escalares. Por esse motivo, nas definições e resultados que aparecem ao longo do Capítulo 1, os espaços vetoriais, quando envolvidos, são reais. Um leitor experiente nos tópicos mencionados acima pode começar a leitura deste texto diretamente no Capítulo 2. Alertamos porém, que na Seção 1.4 além dos resultados bem conhecidos sobre teoria da medida, enunciamos e provamos outros ("menos conhecidos") resultados importantes para justificar satisfatoriamente as provas dadas nos demais capítulos, e colocados aí exatamente para tornar "mais limpas" essas provas. Sua leitura, embora importante, 
pode ser deixada para o momento em que a elas for feita referência. Certamente existem outras definições e resultados que caberiam nesse primeiro capítulo (como por exemplo outras caracterizações de conjunto Lebesgue mensurável de $\mathbb{R}$ ), e alguns que ali estão poderiam ser suprimidos; no entanto, acreditamos que nossa escolha, se não perfeita, ajudará o leitor a compreender melhor o conteúdo dos capítulos seguintes.

Por fim, esperamos que o leitor experimente êxtase semelhante àquele experimentado por quem escreve, em relação às construções elaboradas na tentativa de solução do problema em questão. 

"O valor de praticar com rigor, por algum tempo, uma ciência rigorosa não está propriamente em seus resultados: pois eles sempre serão uma gota infima, ante o mar das coisas dignas de saber. Mas isso produz um aumento de energia, de capacidade dedutiva, de tenacidade; aprende-se a alcançar um fim de modo pertinente. Neste sentido é valioso, em vista de tudo o que se fará depois, ter sido homem de ciência."

Nietzsche 



\section{CAPíTULO 1}

\section{Preliminares}

\subsection{Tópicos de Teoria dos Conjuntos}

Uma compreensão correta sobre números ordinais e números cardinais é o nosso objetivo nesta seção. Todas as definições e resultados são estabelecidos sobre a axiomática ZFC (Zermelo-Fraenkel e o axioma da Escolha). Começamos com a seguinte:

Definição 1.1.1. Sejam $A$ um conjunto e $R$ uma relação binária em $A$. A relação $R$ é chamada de:

(a) reflexiva em $A$ se $a R a$ para todo $a \in A$;

(b) simétrica em $A$ se para todo $a, b \in A, a R b$ implica $b R a$;

(c) antissimétrica em $A$ se para todo $a, b \in A$, se $a R b$ e $b R a$ então $a=b$;

(d) assimétrica em $A$ se para todo $a, b \in A$, se $a R b$ então não vale $b R a$, i.e., $a R b$ e $b R a$ não valem simultaneamente;

(e) transitiva em $A$ se para todo $a, b, c \in A$, se $a R b$ e $b R c$ então $a R c$;

(f) uma relação de equivalência em $A$ se $R$ é reflexiva, simétrica e transitiva em $A$;

(g) uma relação de ordem (parcial) em $A$ se $R$ é reflexiva, antissimétrica e transitiva em $A$;

(h) uma relação de ordem estrita em $A$ se $R$ é assimétrica e transitiva em $A$.

Dada uma relação de ordem $R$ em um conjunto $A$, a relação binária $S$, definida em $A$ por $a S b$ se e somente se $a R b$ e $a \neq b$, é uma (relação de) ordem estrita em $A$. Por outro lado, dada uma relação de ordem estrita $S$ em um conjunto $A$, a relação binária $R$, definida em $A$ por $a R b$ se e somente se $a S b$ ou $a=b$, é uma (relação de) ordem em $A$. 
Como é usual, denotaremos por $\sim$ uma relação de equivalência. Em geral, uma ordem será denotada por $\leq$ ou $\preceq$, e uma ordem estrita por $<$ ou $\prec$.

DeFiniÇão 1.1.2. Dado um conjunto $A$, uma relação de ordem $\leq$ (ou ordem estrita $<$ ) é chamada de linear ou total se dois elementos quaisquer de $A$ são comparáveis, ou seja, para todo $a, b \in A$ temos $a \leq b$ ou $b \leq a$ $(a<b$ ou $b<a)$.

DEFINIÇÃo 1.1.3. Um conjunto (parcialmente) ordenado é um par $(A, \leq)$, no qual $A$ é um conjunto e $\leq$ é uma ordem em $A$; se $\leq$ é uma ordem total em $A$, dizemos que o par $(A, \leq)$ é um conjunto totalmente (ou linearmente) ordenado.

Dados um conjunto $A$ e uma ordem estrita $<$ em $A$, diremos, por abuso de linguagem, que o par $(A,<)$ é um conjunto ordenado (totalmente ordenado, se a ordem $<$ for total); nesse caso fica subentendido que $<$ está no lugar de $\leq$.

OBSERVAÇÃo 1.1.4. Escreveremos $A \subset B$ quando $A$ for um subconjunto de $B$; se $A \subset B$ e $A \neq B$, diremos que $A$ é um subconjunto próprio de $B$. Dados dois conjuntos $A$ e $B, B \backslash A$ denotará a diferença entre $B$ e $A$ (i.e., o conjunto dos elementos de $B$ que não estão em $A$ ), e num contexto em que estiver subentendido algum "conjunto universo" $X$, se $A \subset X$ então a diferença $X \backslash A$ é chamada de complementar de $A$ em $X$ e denotada por $A^{c}$.

DeFiniÇÃo 1.1.5. Seja $(A, \leq)$ um conjunto ordenado. Um subconjunto $B \subset A$ é uma cadeia em $A$ se quaisquer dois elementos de $B$ são comparáveis.

DefiniçÃo 1.1.6. Sejam $(A, \leq)$ um conjunto ordenado, $B$ um subconjunto não vazio de $A$ e $b$ um elemento de $A$. Dizemos que, com respeito à ordem $\leq$ :

(a) $b$ é o elemento mínimo de $B$ se $b \in B$ e $b \leq x$ para todo $x \in B$;

(b) $b$ é um elemento minimal de $B$ se $b \in B$ e não existe $x \in B$ tal que $x \neq b$ e $x \leq b$;

(c) $b$ é o elemento máximo de $B$ se $b \in B$ e $x \leq b$ para todo $x \in B$;

(d) $b$ é um elemento maximal de $B$ se $b \in B$ e não existe $x \in B$ tal que $x \neq b$ e $b \leq x$ 
(e) $b$ é um limite inferior de $B$ se $b \leq x$ para todo $x \in B$;

(f) $b$ é o ínfimo de $B$ (notação: $\inf (B))$ se $b$ é o maior limite inferior de $B$

(g) $b$ é um limite superior de $B$ se $x \leq b$ para todo $x \in B$;

(h) $b$ é o supremo de $B$ (notação: $\sup (B))$ se $b$ é o menor limite superior de $B$.

Lema 1.1.7 (de Zorn). Se toda cadeia em um conjunto parcialmente ordenado $(A, \leq)$ tem um limite superior, então o conjunto $A$ admite um elemento maximal.

DEFINIÇÃo 1.1.8. Um isomorfismo entre dois conjuntos ordenados $(P,<)$ e $(Q, \prec)$ é uma bijeção $h: P \rightarrow Q$ tal que, para todo $a, b \in P$, $a<b$ se e somente se $h(a) \prec h(b)$. Se existe um isomorfismo entre $(P,<)$ e $(Q, \prec)$ nós dizemos que $(P,<)$ e $(Q, \prec)$ são isomorfos.

Não é difícil mostrar que se $(P,<)$ e $(Q, \prec)$ são conjuntos totalmente ordenados e $h: P \rightarrow Q$ é uma bijeção tal que, para todo $a, b \in P, a<b$ implica $h(a) \prec h(b)$, então $h$ é um isomorfismo entre $(P,<)$ e $(Q, \prec)$.

DeFiniÇÃo 1.1.9. Dado um conjunto $x$, o sucessor de $x$ é definido como o conjunto $S(x)=x \cup\{x\}$. O sucessor de $x$ também é denotado por $x+1$.

DEFINIÇÃo 1.1.10. Um conjunto $I$ é chamado indutivo quando

(a) $\emptyset \in I$

(b) se $n \in I$ então $S(n) \in I$.

O axioma do infinito da teoria axiomática ZFC garante a existência de um conjunto indutivo. Isso nos permite estabelecer a seguinte definição:

DEFINIÇÃO 1.1.11. O conjunto dos números naturais, denotado por $\omega$, é por definição a intersecção de todos os conjuntos indutivos (ou seja, $\omega$ é o menor dos conjuntos indutivos).

Dado um conjunto $B$, escreveremos $A \stackrel{\text { def }}{=} B$ para expressar que $A$ é por definição o conjunto $B$ (em geral, $E \stackrel{\text { def }}{=} F$ indicará que E é por definição $\mathrm{F}$ ). 
Como é usual:

$$
\begin{aligned}
& 0 \stackrel{\text { def }}{=} \emptyset \\
& 1 \stackrel{\text { def }}{=} S(0)=0 \cup\{0\}=\{0\}, \\
& 2 \stackrel{\text { def }}{=} S(1)=1 \cup\{1\}=\{0,1\}, \\
& 3 \stackrel{\text { def }}{=} S(2)=2 \cup\{2\}=\{0,1,2\}, \\
& \quad \vdots \\
& S(n)=n \cup\{n\}=\{0,1,2, \ldots, n\}, \text { para todo } n \in \omega,
\end{aligned}
$$

e escrevemos $\omega=\{0,1,2,3, \ldots\}$. A relação binária $\in$ no conjunto $\omega$ é uma ordem estrita total e é a ordem usual em $\omega$.

DeFinição 1.1.12. Uma ordem total $<$ em um conjunto $A$ é chamada uma boa ordem se cada subconjunto não vazio de $A$ tem um elemento mínimo. Se $<$ é uma boa ordem em $A$ então o par $(A,<)$ é chamado de conjunto bem ordenado.

É possível mostrar que a ordem $\in$ dos naturais é uma boa ordem, e portanto $(\omega, \in)$ é um conjunto bem ordenado.

DEFINIÇÃo 1.1.13. Seja $(W,<)$ um conjunto totalmente ordenado. Um subconjunto $S \subset W$ é chamado de um segmento inicial de $W$ se é próprio (i.e., $S \neq W$ ) e se para cada $a \in S$ todo $x<a$ é também elemento de $S$.

DefiniÇÃo 1.1.14. Dois conjuntos $A$ e $B$ são chamados de equipotentes se existe uma bijeção de $A$ em $B$.

DefiniÇÃo 1.1.15. Um conjunto $A$ é finito se é equipotente a algum $n \in \omega$ (nesse caso dizemos que $A$ tem $n$ elementos), infinito se não é finito, e enumerável se é finito ou equipotente a $\omega$.

DEFINIÇÃo 1.1.16. Um conjunto $T$ é chamado de transitivo se cada elemento de $T$ é um subconjunto de $T$.

Todo número natural é um conjunto transitivo. De fato, se $n \in \omega$ e $p \in m \in n$ então $p \in n$, o que mostra que $m \subset n$.

DEFINIÇÃo 1.1.17. Um conjunto $\alpha$ é um número ordinal (ou um ordinal) se $\alpha$ é transitivo e bem ordenado por $\epsilon$. 
DefiniÇÃo 1.1.18. Dados dois números ordinais $\alpha$ e $\beta$, definimos $\alpha<\beta$ se e somente se $\alpha \in \beta$.

ProposiçÃo 1.1.19.

(a) $0=\emptyset$ é um número ordinal;

(b) se $\alpha$ é um ordinal e $\beta \in \alpha$ então $\beta$ é um ordinal;

(c) para quaisquer ordinais $\alpha$ e $\beta, \alpha \subset \beta$ se somente se $\alpha \in \beta$ ou $\alpha=\beta$;

(d) se $\alpha$ e $\beta$ são dois ordinais então, $\alpha \leq \beta$ se e somente se $\alpha \subset \beta$.

Corolário 1.1.20.

(a) Se $\alpha, \beta$ e $\gamma$ são ordinais tais que $\alpha<\beta$ e $\beta<\gamma$ então $\alpha<\gamma$;

(b) se $\alpha, \beta$ são ordinais então $\alpha<\beta$ e $\beta<\alpha$ não valem simultaneamente;

(c) dados ordinais $\alpha$ e $\beta$, ou $\alpha<\beta$ ou $\alpha=\beta$ ou $\beta<\alpha$;

(d) cada conjunto não vazio de números ordinais tem um elemento mínimo com respeito à ordem $<$;

(e) para cada ordinal $\alpha, \alpha=\{\beta: \beta<\alpha\}$;

(f) se $C$ é um conjunto ${ }^{1}$ não vazio de números ordinais e $\alpha=\bigcap_{\beta \in C} \beta$, então $\alpha$ é um ordinal e $\alpha$ é o elemento mínimo de $C$;

(g) se $C$ é um conjunto não vazio de números ordinais e $\alpha=\bigcup_{\beta \in C} \beta$, então $\alpha$ é um ordinal e $\alpha=\sup (C)$;

(h) para cada ordinal $\alpha, \alpha+1$ é um ordinal e $\alpha+1=\inf \{\beta: \alpha<\beta\}$;

(i) para cada conjunto de ordinais $C$ existe um ordinal $\alpha \notin C$.

Dos itens (a) da Proposição 1.1.19 e (h) do Corolário 1.1.20 concluímos que todo número natural é um número ordinal; na verdade é possível mostrar que os naturais são exatamente os ordinais finitos. Assim, pelo item (g) do Corolário 1.1.20, o conjunto $\omega$ também é número ordinal; mais do que isso, $\omega$ é o primeiro ordinal enumerável infinito.

DEFINIÇÃo 1.1.21. Um número ordinal $\alpha$ é chamado um ordinal sucessor se $\alpha=\beta+1$ para algum ordinal $\beta$, caso contrário $\alpha$ é chamado de ordinal limite.

\footnotetext{
${ }^{1} \mathrm{Na}$ verdade, dada uma propriedade $\varphi(x)$ satisfeita por pelo menos um ordinal, então existe um número ordinal que é o menor ordinal que satisfaz $\varphi(x)$.
} 
Se $\alpha$ é um ordinal limite então $\alpha=\sup \{\beta: \beta<\alpha\}=\bigcup_{\beta \in \alpha} \beta$; o natural 0 será considerado um ordinal limite e $0=\sup (\emptyset)$.

TeOrema 1.1.22. Todo conjunto bem ordenado é isomorfo a um único número ordinal.

DeFiniÇÃo 1.1.23. Se $(A,<)$ é um conjunto bem ordenado definimos o tipo (de ordem) de $(A,<)$, denotado por $\operatorname{tipo}(A,<)$, como o único ordinal isomorfo a $(A,<)$.

DEFINIÇÃo 1.1.24. Um número ordinal $\alpha$ é chamado de número cardinal (ou apenas, cardinal) se não existe $\beta<\alpha$ tal que $\alpha$ é equipotente a $\beta$.

Todo conjunto infinito pode ser bem ordenado de muitas maneiras diferentes, e embora cada conjunto bem ordenado seja isomorfo a um único número ordinal, ele pode ser equipotente a infinitos números ordinais, por exemplo, um conjunto enumerável infinito é equipotente a $\omega$, a $\omega+1$, a $\omega+2$, etc. Dada uma propriedade $\varphi(x)$ satisfeita por pelo menos um número ordinal, sabemos que existe o menor ordinal que a satisfaz. Essas observações possibilitam-nos estabelecer a seguinte:

DEFiniÇão 1.1.25. Seja $(A,<)$ um conjunto bem ordenado. A cardinalidade de $A$, denotada por $|A|$ (ou card $(A)$ ), é o menor número ordinal equipotente a $A$.

ProposiçÃo 1.1.26.

(a) Se $\kappa$ é um cardinal então $\kappa=\operatorname{card}(\kappa)$;

(b) $|A|$ é um número cardinal para todo conjunto $A$;

(c) dados dois conjuntos $X$ e $Y,|X| \leq|Y|$ se e somente se existe uma função injetora $f: X \rightarrow Y$;

(d) dados dois conjuntos $X$ e $Y, X \neq \emptyset,|X| \leq|Y|$ se e somente se existe um função sobrejetora $g: Y \rightarrow X$.

Observação 1.1.27. Se $A$ e $B$ são dois conjuntos então o conjunto de todas as funções de $A$ em $B$ será denotado por $B^{A}$.

Proposição 1.1.28. Dados dois conjuntos $A$ e $B$, definimos:

(a) $|A|+|B|=|(A \times\{0\}) \cup(B \times\{1\})|$;

(b) $|A| \cdot|B|=|A \times B|$; 
(c) $|A|^{|B|}=\left|A^{B}\right|$.

Teorema 1.1.29 (Cantor). Para todo conjunto $X$ temos $|X|<|\mathcal{P}(X)|$.

Teorema 1.1.30. $|\mathcal{P}(X)|=2^{|X|}$ para todo conjunto $X$.

TeOrema 1.1.31. $|\mathbb{R}|=|\mathcal{P}(\omega)|$

Note que todo número natural é um cardinal e $\omega$ é o menor número cardinal infinito. Observamos ainda que todo cardinal infinito é um ordinal limite, e os números ordinais infinitos que são cardinais são chamados de $\operatorname{alephs(\aleph ).~Através~da~Proposição~} 1.1 .32$ a seguir e do princípio de recursão transfinita (vide $[\mathbf{1 6}]$ ), podemos a cada número ordinal $\alpha$ associar um cardinal $\aleph_{\alpha}$, obtendo assim uma seqüência (indexada pelos ordinais) de todos os alephs.

ProposiçÃo 1.1.32.

(a) Para cada cardinal $\kappa$ existe um número cardinal maior do que $\kappa$;

(b) se $X$ é um conjunto de cardinais então $\sup (X)$ é um número cardinal.

Para cada cardinal $\kappa$, o sucessor cardinal de $\kappa$, denotado por $\kappa^{+}$, é o menor número cardinal maior do $\kappa$.

DefiniçÃo 1.1.33. Seja $\alpha$ um número ordinal. Definimos:

$$
\left\{\begin{array}{l}
\aleph_{0}=\omega ; \\
\aleph_{\alpha+1}=\aleph_{\alpha}^{+} ; \\
\aleph_{\alpha}=\sup \left\{\aleph_{\beta}: \beta<\alpha\right\}=\bigcup_{\beta<\alpha} \aleph_{\beta}, \text { se } \alpha \text { é um ordinal limite. }
\end{array}\right.
$$

Em que claramente $\aleph_{\alpha+1}$ é um sucessor cardinal, e $\aleph_{\alpha}$ é um dito um cardinal limite se $\alpha$ é um ordinal limite.

TeOrema 1.1.34. Se $\alpha$ e $\beta$ são números ordinais e $n$ é um número natural então:

(a) $n+\aleph_{\alpha}=n \cdot \aleph_{\alpha}=\aleph_{\alpha}$;

(b) $\aleph_{\alpha}+\aleph_{\beta}=\aleph_{\alpha} \cdot \aleph_{\beta}=\max \left\{\aleph_{\alpha}, \aleph_{\beta}\right\}$.

Para cada conjunto infinito $A$ existe um único número ordinal $\alpha$ tal que $\operatorname{card}(A)=\aleph_{\alpha}$; em particular, se $\kappa$ é número cardinal infinito então existe 
um único ordinal $\alpha$ tal que $\kappa=\aleph_{\alpha}$. Se $\alpha$ e $\beta$ são números ordinais com $\alpha<\beta$ então $\aleph_{\alpha}<\aleph_{\beta}$, i.e., a seqüência de todos os alephs é crescente. Observamos que, conforme definição anterior, um conjunto enumerável é um conjunto cuja cardinalidade (ou cujo número cardinal) é menor ou igual a $\aleph_{0}$; além disso $\aleph_{0}$ é o menor cardinal infinito enumerável e $\aleph_{1}$ é o menor cardinal infinito não enumerável. Como conseqüência dos Teoremas 1.1.29 e 1.1.30 temos que $\kappa<2^{\kappa}$ para todo número cardinal $\kappa$, e portanto, dos Teoremas 1.1 .30 e 1.1.31 concluímos que $|\mathbb{R}|=|\mathcal{P}(\omega)|=2^{|\omega|}=2^{\aleph_{0}}>\aleph_{0}$. O número cardinal $2^{\aleph_{0}}$ é chamado de a cardinalidade do continuum (ou o número cardinal de $\mathbb{R}$ ), e a conhecida Hipótese do Continuum afirma que $2^{\aleph_{0}}=\aleph_{1}$. A conjectura $2^{\aleph_{\alpha}}=\aleph_{\alpha+1}$, para todo ordinal $\alpha$, é chamada de Hipótese do Continuum Generalizada $(\mathrm{GCH})^{2}$, e assim como a Hipótese do Continuum, GCH é independente dos axiomas de ZFC, ou seja, GCH não pode ser provada nem refutada a partir dos axiomas de ZFC.

\subsection{Um Mínimo de Álgebra Linear e Espaços Métricos}

Dados um espaço vetorial $V$ e um subespaço $W \subset V$, a relação $\sim$, definida por $u \sim v$ se $u-v \in W$, para cada $u, v \in V$, é uma relação de equivalência em $V$. Para um vetor $u \in V$, indicamos por $[u]$ a sua classe de equivalência. $\mathrm{O}$ conjunto

$$
V / W=V / \sim=\{[u]: u \in V\},
$$

munido com as operações:

(i) $[u]+[v]=[u+v]$, para todo $u, v \in V$ e

(ii) $\lambda[u]=[\lambda u]$, para todo $\lambda \in \mathbb{R}$, e todo $u \in V$,

é um espaço vetorial sobre $\mathbb{R}$, chamado de espaço quociente, cujo vetor nulo é o subespaço $W$.

Para espaços vetoriais $U$ e $V$ denotamos por $\mathrm{L}(U, V)$ o conjunto de todas as transformações lineares de $U$ em $V . \mathrm{L}(U, V)$ é um subespaço do espaço vetorial de todas as funções de $U$ em $V$. Um funcional linear é uma transformação linear $f: U \rightarrow \mathbb{R}$, e o espaço vetorial $\mathrm{L}(U, \mathbb{R})$, indicado por $U^{*}$, é chamado de espaço dual algébrico de $U$.

\footnotetext{
${ }^{2}$ Do equivalente em inglês, Generalized Continuum Hypothesis.
} 
DeFiniÇÃo 1.2.1. Sejam $U$ e $V$ espaços vetoriais sobre $\mathbb{R}$ e $T: U \rightarrow V$ uma transformação linear. A transformação linear $T^{*}: V^{*} \rightarrow U^{*}$ dada por $T^{*}(g)(u)=g(T(u))$, para todo $g \in V^{*}$ e todo $u \in U$, é chamada de transposta de $T$.

Proposição 1.2.2. Sejam $U$ e $V$ espaços vetoriais sobre $\mathbb{R}$ e $T: U \rightarrow V$ uma transformação linear. Se $U_{o}$ e $V_{o}$ são subespaços de $U$ e $V$ respectivamente, com $T\left(U_{o}\right) \subset V_{o}$, então a aplicação $\Phi: U / U_{o} \rightarrow V / V_{o}$, definida por $\Phi([u])=[T(u)]$, é linear. Dizemos que $\Phi$ é a aplicação linear induzida por $T$ em $U / U_{o}$, ou simplesmente que $T$ induz $\Phi$.

\section{DEMONSTRAÇÃO.}

- $\Phi$ está bem definida.

Sejam $u$ e $v$ elementos arbitrários de $U$. Se $[u]=[v]$ então $u-v \in U_{o}$, e portanto $T(u-v) \in T\left(U_{o}\right) \subset V_{o}$, ou seja, $T(u)-T(v) \in V_{o}$; logo $[T(u)]=[T(v)]$, isto é, $\Phi([u])=\Phi([v])$.

- $\Phi$ é linear.

Se $[u],[v] \in U / U_{o}$ e $\alpha$ é um número real, então:

$$
\begin{array}{r}
\Phi(\alpha[u]+[v])=\Phi([\alpha u+v])=[T(\alpha u+v)]=[\alpha T(u)+T(v)] \\
=\alpha[T(u)]+[T(v)]=\alpha \Phi([u])+\Phi([v])
\end{array}
$$

Com esta proposição finalizamos as idéias a respeito de álgebra linear que queríamos destacar. A partir da próxima definição, até o fim da seção, colocamos em foco algumas noções sobre espaços métricos.

DEFiniÇÃo 1.2.3. Uma métrica em um conjunto $M$ é uma função $d: M \times M \rightarrow \mathbb{R}$ tal que para quaisquer $x, y, z \in M$ temos satisfeitas as seguintes condições:

(a) $d(x, y) \geq 0$;

(b) $d(x, y)=0$ se e somente se $x=y$;

(c) $d(x, y)=d(y, x)$;

(d) $d(x, y) \leq d(x, z)+d(z, y)$.

DefiniÇÃo 1.2.4. Um espaço métrico é um par $(M, d)$, em que $M$ é um conjunto e $d$ é uma métrica em $M$. 
DeFinição 1.2.5. Seja $E$ um espaço vetorial real. Uma norma em $E$ é uma aplicação $\|\cdot\|: E \rightarrow \mathbb{R}$ que associa a cada vetor $x \in E$ o número real $\|x\|$, chamado de a norma de $x$, de modo que, para quaisquer $x, y \in E \mathrm{e}$ $\lambda \in \mathbb{R}$, sejam satisfeitas as seguintes condições:

(a) $\|x\| \geq 0$;

(b) $\|x\|=0$ se e somente se $x=0$;

(c) $\|\lambda x\|=|\lambda|\|x\|$;

(d) $\|x+y\| \leq\|x\|+\|y\|$.

DEFINIÇÃo 1.2.6. Um espaço vetorial real normado é um par $(E,\|\cdot\|)$, no qual $E$ é um espaço vetorial sobre $\mathbb{R}$ e $\|\cdot\|$ é uma norma em $E$.

Todo espaço vetorial normado $(E,\|\cdot\|)$ torna-se de modo natural um espaço métrico $(E, d)$, fazendo $d(x, y)=\|x-y\|$, para todo $x, y \in E$; a aplicação $d$ é uma métrica em $E$ dita proveniente da (ou associada à, ou determinada pela) norma $\|\cdot\|$. Assim, para um espaço normado, entenderemos sempre que o espaço métrico correspondente está munido com a métrica proveniente da norma.

DefiniÇão 1.2.7. Sejam $\left(M, d_{M}\right)$ e $\left(N, d_{N}\right)$ dois espaços métricos. Uma aplicação $f: M \rightarrow N$ chama-se uma imersão isométrica quando

$$
d_{N}(f(x), f(y))=d_{M}(x, y)
$$

para quaisquer $x, y \in M$. Neste caso, dizemos também que $f$ preserva distância.

Observamos que uma imersão isométrica $f: M \rightarrow N$ é sempre injetora, uma vez que, para quaisquer $x, y \in M$ satisfazendo $f(x)=f(y)$, temos $d_{M}(x, y)=d_{N}(f(x), f(y))=0$, e conseqüentemente $x=y$.

DefiniÇÃo 1.2.8. Sejam $\left(M, d_{M}\right)$ e $\left(N, d_{N}\right)$ espaços métricos. Uma imersão isométrica $f: M \rightarrow N$ sobrejetora é chamada de uma isometria.

Note que toda imersão isométrica $f: M \rightarrow N$ define uma isometria de $\mathrm{M}$ sobre o subespaço métrico $f(M) \subset N$. Observe ainda que para dois espaços vetoriais normados $\left(E,\|\cdot\|_{E}\right)$ e $\left(F,\|\cdot\|_{F}\right)$, uma aplicação linear $T: E \rightarrow F$ é uma imersão isométrica se $\|T(x)\|_{F}=\|x\|_{E}$ para todo $x \in E$; neste caso $T$ é chamada de imersão isométrica linear. Além disso, se $T$ é sobrejetora então $T$ é dita uma isometria linear. 
DEFINIÇÃo 1.2.9. Seja $(M, d)$ um espaço métrico. Um subconjunto $Y \subset M$ é chamado de limitado se existe $k \in \mathbb{R}, k \geq 0$, tal que $d(x, y) \leq k$ para todo $x, y \in Y$. Uma função $f: X \rightarrow M, X$ um conjunto arbitrário, é dita limitada quando a imagem de $f$ é um subconjunto limitado de $M$.

Para finalizar esta seção, lembramos que uma seqüência de Cauchy em um espaço métrico $(M, d)$ é uma seqüência $\left(x_{n}\right)_{n \geq 1}$ de elementos de $M$ tal que, dado $\varepsilon>0$ existe um número natural $n_{0}$ tal que $d\left(x_{m}, x_{n}\right)<\varepsilon$ para todo $m, n>n_{0}$. Lembramos ainda que toda seqüência em $M$ convergente é uma seqüência de Cauchy, mas não necessariamente uma seqüência de Cauchy em $M$ é convergente em $M$. Quando em um espaço métrico $(M, d)$ toda seqüência de Cauchy é convergente, dizemos que $(M, d)$ é um espaço métrico completo. Em particular, um espaço normado $(E,\|\cdot\|)$ é completo se o espaço métrico correspondente $(E, d)$ é completo.

\subsection{Tópicos de Análise Funcional}

Diante do que foi dito no final da seção anterior, começamos esta nova seção com a seguinte definição:

DEFINIÇÃo 1.3.1. Um espaço vetorial real normado $(E,\|\cdot\|)$ é chamado de espaço de Banach se $(E,\|\cdot\|)$ é completo.

Se $(E,\|\cdot\|)$ é um espaço normado então todo subespaço $V \subset E$ pode ser encarado como um espaço normado cuja norma é a restrição da norma de $E$. Além disso, se $E$ é um espaço de Banach, sabemos que um subespaço $V$ de $E$ será um espaço de Banach se e somente se $V$ for fechado em $E$.

A seguir definimos quando uma aplicação linear entre espaços vetoriais normados é dita limitada. Segundo a definição 1.2.9, uma função $f: X \rightarrow M$, na qual $X$ é um conjunto arbitrário e $M$ é um espaço métrico, é limitada quando sua imagem é um conjunto limitado. No caso de uma aplicação linear entre dois espaços normados, o termo "limitada" será usado num sentido diferente. Veremos que uma aplicação linear entre espaços normados $\left(E,\|\cdot\|_{E}\right)$ e $\left(F,\|\cdot\|_{F}\right)$ é dita limitada se sua restrição à bola unitária de $E$ é limitada no sentido da definição 1.2.9. 
Proposição 1.3.2. Sejam $\left(E,\|\cdot\|_{E}\right)$ e $\left(F,\|\cdot\|_{F}\right)$ espaços vetoriais reais normados e $T: E \rightarrow F$ uma aplicação linear. Então são equivalentes as seguintes afirmações:

(a) Té contínua;

(b) $T$ é contínua na origem;

(c) Té limitada em alguma vizinhança da origem, i.e., existem $c \geq 0$ e uma vizinhança $V$ da origem tais que $\|T(x)\|_{F} \leq c$ para todo $x \in V$;

(d) $T$ é limitada na bola unitária de $E$, i.e., existe $c \geq 0$ tal que $\|T(x)\|_{F} \leq$ c para todo $x \in E$, com $\|x\|_{E} \leq 1 ;$

(e) $T$ é limitada na esfera unitária de $E$, i.e., existe $c \geq 0$ tal que $\|T(x)\|_{F} \leq$ c para todo $x \in E$, com $\|x\|_{E}=1 ;$

(f) existe $c \geq 0$ tal que $\|T(x)\|_{F} \leq c\|x\|_{E}$ para todo $x \in E$;

(g) $T$ é Lipschitziana.

\section{DEMONSTRAÇÃO.}

- $(a) \Rightarrow(b)$. Imediato.

- $(b) \Rightarrow(c)$. Se $T$ é contínua na origem então existe $\delta>0$ tal que $\|T(x)-T(0)\|_{F}=\|T(x)\|_{F}<1$, para todo $x \in E$ com $\|x\|_{E}<\delta$, ou seja, $T$ é limitada na bola aberta de centro na origem e raio $\delta$.

- $(c) \Rightarrow(d)$. Se $T$ é limitada em alguma vizinhança da origem então existem $r>0$ e $c \geq 0$ tais que $\|T(x)\|_{F} \leq c$ para todo $x \in E$ com $\|x\|_{E} \leq r$. Seja $x \in E$ com $\|x\|_{E} \leq 1$. Então $\|r x\|_{E} \leq r$, e portanto $\|T(x)\|_{F}=\frac{1}{r}\|T(r x)\|_{F} \leq \frac{c}{r}$, para todo $x \in E$ com $\|x\|_{E} \leq 1$.

- $(d) \Rightarrow(e)$. Imediato.

- $(e) \Rightarrow(f)$. Seja $c \geq 0$ tal que $\|T(x)\|_{F} \leq c$ para todo $x \in E$, com $\|x\|_{E}=1$. Afirmamos que $\|T(x)\|_{F} \leq c\|x\|_{E}$ para todo $x \in E$. Com efeito, se $x=0$ então a desigualdade vale trivialmente. Por outro lado, se $x \neq 0$ então o vetor $\frac{x}{\|x\|_{E}}$ tem norma unitária, logo

$$
\frac{\|T(x)\|_{F}}{\|x\|_{E}}=\left\|\frac{T(x)}{\|x\|_{E}}\right\|_{F}=\left\|T\left(\frac{x}{\|x\|_{E}}\right)\right\|_{F} \leq c,
$$

ou seja, $\|T(x)\|_{F} \leq c\|x\|_{E}$. 
- $(f) \Rightarrow(g)$. Seja $c \geq 0$ tal que $\|T(x)\|_{F} \leq c\|x\|_{E}$ para todo $x \in E$. Para todo $x, y \in E$ temos $\|T(x)-T(y)\|_{F}=\|T(x-y)\|_{F} \leq c\|x-y\|_{E}$, e portanto $c$ é uma constante de Lipschitz para $T$.

- $(g) \Rightarrow(a)$. Imediato.

DeFiniçÃo 1.3.3. Sejam $\left(E,\|\cdot\|_{E}\right)$ e $\left(F,\|\cdot\|_{F}\right)$ espaços vetoriais reais normados. Uma aplicação linear $T: E \rightarrow F$ é dita limitada se satisfaz uma das (e portanto todas) condições que aparecem no enunciado da Proposição 1.3.2.

Observamos que se $T: E \rightarrow F$ é uma aplicação linear não nula então sua imagem nunca é um conjunto limitado. De fato, se $T$ é não nula então existe $x \in E \operatorname{com} T(x) \neq 0$. Como $\|T(\alpha x)\|=|\alpha|\|T(x)\|$ para todo $\alpha \in \mathbb{R}$, temos que $\|T(\alpha x)\| \longrightarrow+\infty$ quando $|\alpha| \longrightarrow+\infty$, e portanto a imagem de $T$ não é limitada. Assim, quando associado a uma aplicação linear, o termo "limitada" só pode ser entendido no sentido da definição 1.3.3.

Se $\left(E,\|\cdot\|_{E}\right)$ e $\left(F,\|\cdot\|_{F}\right)$ são espaços vetoriais normados sobre $\mathbb{R}$, denotamos por $\operatorname{Lin}(E, F)$ o conjunto de todas as aplicações lineares limitadas $T: E \rightarrow F$. Não é difícil mostrar que $\operatorname{Lin}(E, F)$ é um subespaço vetorial de $\mathrm{L}(E, F)$, o espaço vetorial de todas as aplicações lineares de $E$ em $F$.

DefiniÇão 1.3.4. Sejam $\left(E,\|\cdot\|_{E}\right)$ e $\left(F,\|\cdot\|_{F}\right)$ espaços vetoriais reais normados e $T \in \operatorname{Lin}(E, F)$. Definimos a norma de $T$ por

$$
\|T\|=\sup \left\{\|T(x)\|_{F}: x \in E \text { e }\|x\|_{E} \leq 1\right\},
$$

ou sinteticamente,

$$
\|T\|=\sup _{\|x\|_{E} \leq 1}\|T(x)\|_{F}
$$

A aplicação $\|\cdot\|: \operatorname{Lin}(E, F) \rightarrow \mathbb{R}$ definida acima é de fato uma norma, e é possível mostrar que:

$$
\begin{aligned}
\|T\| & =\sup \left\{\|T(x)\|_{F}: x \in E \text { e }\|x\|_{E}=1\right\} \\
& =\inf \left\{c \geq 0:\|T(x)\|_{F} \leq c\|x\|_{E}, \text { para todo } x \in E\right\} .
\end{aligned}
$$

Proposição 1.3.5. Se $\left(E,\|\cdot\|_{E}\right)$ e $\left(F,\|\cdot\|_{F}\right)$ espaços vetoriais reais normados e $T \in \operatorname{Lin}(E, F)$ então $\|T(x)\|_{F} \leq\|T\|\|x\|_{E}$ para todo $x \in E$. 
Proposição 1.3.6. Sejam $\left(E,\|\cdot\|_{E}\right) e\left(F,\|\cdot\|_{F}\right)$ espaços vetoriais normados sobre $\mathbb{R}$. Se $\left(F,\|\cdot\|_{F}\right)$ é um espaço de Banach então $\operatorname{Lin}(E, F)$, com a norma definida como em (1.3.4), também é um espaço de Banach.

DemonstraÇÃo. Vide [17].

Se $E$ é um espaço vetorial sobre $\mathbb{R}$, vimos que $E^{*}=\mathrm{L}(E, \mathbb{R})$, o conjunto de todos os funcionais lineares $f: E \rightarrow \mathbb{R}$, é chamado de espaço dual algébrico de $E$. Dado um espaço vetorial real normado $\left(E,\|\cdot\|_{E}\right)$, um funcional linear $f: E \rightarrow \mathbb{R}$ é dito limitado se $f \in \operatorname{Lin}(E, \mathbb{R})$, ou seja, se $f$ é uma aplicação linear limitada. O espaço normado $\operatorname{Lin}(E, \mathbb{R})$ é chamado de dual topológico de $E$. Note que $\operatorname{Lin}(E, F)$ é subespaço vetorial de $E^{*}$. Como neste texto (e em geral) nos preocupamos apenas com os funcionais lineares limitados, chamaremos o dual topológico de $E$ (simplesmente) de dual de $E$, e por abuso de notação, a partir de agora, usaremos $E^{*}$ para indicar o dual de $E$. Assim, dado um espaço vetorial real normado $(E,\|\cdot\|)$,

$$
\begin{gathered}
E^{*}=\{\alpha: E \rightarrow \mathbb{R}: \alpha \text { é funcional linear limitado }\}=\operatorname{Lin}(E, \mathbb{R}) \text { e } \\
\|\alpha\|=\sup \left\{|\alpha(x)|: x \in E \text { e }\|x\|_{E} \leq 1\right\}, \text { para todo } \alpha \in E^{*} .
\end{gathered}
$$

Uma vez que $\mathbb{R}$ é completo temos, pela Proposição 1.3.6, que $E^{*}$ é um espaço de Banach sobre $\mathbb{R}$, para todo espaço vetorial real normado $(E,\|\cdot\|)$.

A seguir, dados um espaço normado real $(X,\|\cdot\|)$ e um subespaço fechado $Y \subset X$, definimos uma norma no espaço quociente $X / Y$.

Proposição 1.3.7. Seja $Y$ um subespaço fechado de um espaço normado $(X,\|\cdot\|)$. A aplicação $\|\cdot\|_{o}: X / Y \rightarrow \mathbb{R}$, definida por

$$
\|[x]\|_{o}=\inf \{\|z\|: z \in[x]\}
$$

para todo $x \in X$, é uma norma no espaço quociente $X / Y$.

\section{DemonstraÇÃo.}

- A aplicação está bem definida.

$$
\begin{aligned}
& \text { Sejam }[x],[y] \in X / Y \text { com }[x]=[y] \text {. Então } \\
& \qquad\|[x]\|_{o}=\inf \{\|z\|: z \in[x]\}=\inf \{\|z\|: z \in[y]\}=\|[y]\|_{o} .
\end{aligned}
$$


- $\|[x]\|_{o} \geq 0$ para todo $x \in X$.

Se $[x] \in X / Y$ então $0 \leq\|z\|$, para todo $z \in[x]$, e portanto

$$
\|[x]\|_{o}=\inf \{\|z\|: z \in[x]\} \geq 0 .
$$

- Se $\|[x]\|_{o}=0$ então $[x]=[0]$.

Se $[x] \in X / Y \operatorname{com}\|[x]\|_{o}=0$, então inf $\{\|z\|: z \in[x]\}=0 ; \operatorname{logo}$ existe uma seqüência $\left(x_{n}\right)_{n \geq 1}$, com $x_{n} \in[x]$ para todo $n \geq 1$, tal que $\left\|x_{n}\right\| \longrightarrow 0$. Para cada $n \geq 1$, existe $y_{n} \in Y$ tal que $x_{n}=x+y_{n}$; portanto $\left\|x+y_{n}\right\| \longrightarrow 0$, o que implica $x+y_{n} \longrightarrow 0$, i.e., $y_{n} \longrightarrow 0-x$. Como $\left(y_{n}\right)_{n \geq 1}$ é uma seqüência de elementos de $Y$, e $Y$ é um subespaço fechado de $X$, segue que $0-x \in Y$, ou seja, $[x]=[0]$.

- $\|\lambda x\|_{o}=|\lambda|\|[x]\|_{o}$ para todo $\lambda \in \mathbb{R}$ e $x \in X$.

Sejam $[x] \in X / Y$ e $\lambda \in \mathbb{R}$. Se $\lambda=0$ então a igualdade vale trivialmente para todo $x \in X$. Suponhamos $\lambda \neq 0$ e seja $x \in X$ arbitrário. Então $\|\lambda[x]\|_{o}=\|[\lambda x]\|_{o}=\inf \{\|z\|: z \in[\lambda x]\}$. Fazendo $z=\lambda x+y$, $y \in Y$, temos $\|z\|=|\lambda|\|k\|$, em que $k=x+\frac{1}{\lambda} y$. Daí,

$$
\|\lambda[x]\|_{o}=\inf \{|\lambda|\|k\|: k \in[x]\}=|\lambda| \inf \{\|k\|: k \in[x]\}=|\lambda|\|[x]\|_{o} .
$$

- $\|[x]+[y]\|_{o} \leq\|[x]\|_{o}+\|[y]\|_{o}$.

Sejam $[x],[y] \in X / Y$. Dado $\varepsilon>0$, existem $\alpha \in[x]$ e $\beta \in[y]$ tais que $\|\alpha\| \leq\|[x]\|_{o}+\frac{\varepsilon}{2}$ e $\|\beta\| \leq\|[y]\|_{o}+\frac{\varepsilon}{2}$. Como $\alpha \in[x]$ e $\beta \in[y]$, temos $\alpha+\beta \in[x+y]$. Assim,

$$
\|[x]+[y]\|_{o}=\|[x+y]\|_{o} \leq\|\alpha+\beta\| \leq\|\alpha\|+\|\beta\| \leq\|[x]\|_{o}+\|[y]\|_{o}+\varepsilon,
$$

ou seja, $\|[x]+[y]\|_{o} \leq\|[x]\|_{o}+\|[y]\|_{o}+\varepsilon$, para todo $\varepsilon>0$. Portanto,

$$
\|[x]+[y]\|_{o} \leq\|[x]\|_{o}+\|[y]\|_{o} .
$$

É possível mostrar que se $(X,\|\cdot\|)$ é um espaço de Banach e $Y$ é um subespaço fechado de $X$ então $\left(X / Y,\|\cdot\|_{o}\right)$ também é um espaço de Banach.

Definição 1.3.8. Sejam $\left(E,\|\cdot\|_{E}\right)$ e $\left(F,\|\cdot\|_{F}\right)$ espaços de Banach. Uma aplicação linear limitada $\mathfrak{q}: E \rightarrow F$ é chamada de uma aplicação quociente se ela é sobrejetora e, para todo $x \in E$,

$$
\|\mathfrak{q}(x)\|_{F}=\inf \left\{\|z\|_{E}: z \in E \text { e } \mathfrak{q}(z)=\mathfrak{q}(x)\right\} .
$$


Isso equivale a dizer que $\mathfrak{q}$ induz uma isometria linear do espaço quociente de Banach $E / \operatorname{Ker}(\mathfrak{q})$ sobre $F$. Mais especificamente, a aplicação linear limitada $\mathfrak{q}: E \rightarrow F$ é uma aplicação quociente se a aplicação

$$
E / \operatorname{Ker}(\mathfrak{q}) \ni[x] \longmapsto \mathfrak{q}(x) \in F
$$

é uma isometria linear.

DeFiniçÃo 1.3.9. Seja $(X,\|\cdot\|)$ um espaço vetorial normado. Uma família $\left(x_{i}\right)_{i \in I}$ de vetores de $X$ é dita somável quando existe $\alpha \in X$ tal que, para todo $\varepsilon>0$, existe um subconjunto finito $F_{\varepsilon} \subset I$ tal que

$$
\left\|\alpha-\sum_{i \in F} x_{i}\right\|<\varepsilon
$$

para todo subconjunto finito $F \subset I \operatorname{com} F \supset F_{\varepsilon}$. O vetor $\alpha \in X$, quando existe, é único e é chamado de a soma da família $\left(x_{i}\right)_{i \in I}$; escrevemos

$$
\alpha=\sum_{i \in I} x_{i}
$$

Proposição 1.3.10. Sejam $\left(X,\|\cdot\|_{X}\right)$ e $\left(Y,\|\cdot\|_{Y}\right)$ espaços vetoriais reais normados. Se $T: X \rightarrow Y$ é uma aplicação linear limitada e se $\left(x_{i}\right)_{i \in I}$ é uma família somável em $X$ então

$$
T\left(\sum_{i \in I} x_{i}\right)=\sum_{i \in I} T\left(x_{i}\right) .
$$

Demonstração. Seja $\varepsilon>0$. Se $\left(x_{i}\right)_{i \in I}$ é uma família somável em $X$ então existem $\alpha=\sum_{i \in I} x_{i} \in X$ e $F_{\varepsilon} \subset I$ finito tais que

$$
\left\|\alpha-\sum_{i \in F} x_{i}\right\|_{X}<\frac{\varepsilon}{\|T\|}
$$

para todo subconjunto finito $F \subset I$, com $F \supset F_{\varepsilon}$. Uma vez que $T$ é uma aplicação linear limitada e $F$ é finito, temos:

$\left\|T(\alpha)-\sum_{i \in F} T\left(x_{i}\right)\right\|_{Y}=\left\|T\left(\alpha-\sum_{i \in F} x_{i}\right)\right\|_{Y} \leq\|T\|\left\|\alpha-\sum_{i \in F} x_{i}\right\|_{X}<\|T\| \frac{\varepsilon}{\|T\|}=\varepsilon$, para todo $F$ finito com $F_{\varepsilon} \subset F \subset I$. Portanto a família $\left(T\left(x_{i}\right)\right)_{i \in I}$ é somável e $T(\alpha)=\sum_{i \in I} T\left(x_{i}\right)$, i.e.,

$$
T\left(\sum_{i \in I} x_{i}\right)=\sum_{i \in I} T\left(x_{i}\right) .
$$


Lembramos que $\overline{\mathbb{R}}=\mathbb{R} \cup\{-\infty,+\infty\}$ é chamado de sistema de números reais estendido, ou simplesmente de reta estendida. Uma descrição das operações algébricas em $\overline{\mathbb{R}}$ e suas propriedades podem ser encontradas, entre outros, em $[\mathbf{2 2}]$ ou $[\mathbf{3}]$.

DEFINIÇÃo 1.3.11. Seja $\left(x_{i}\right)_{i \in I}$ uma família de números reais não negativos. Definimos a soma de $\left(x_{i}\right)_{i \in I}$ por

$$
\sum_{i \in I} x_{i}=\sup \left\{\sum_{i \in F} x_{i}: F \subset I, F \text { finito }\right\} \in \overline{\mathbb{R}} .
$$

OBSERVAÇÃo 1.3.12. Se $\left(x_{i}\right)_{i \in I}$ é uma família de números reais não negativos e $\phi: J \rightarrow I$ é uma bijeção então

$$
\sum_{i \in I} x_{i}=\sum_{j \in J} x_{\phi(j)}
$$

De fato, para cada $E \subset J$ finito, como $\left.\phi\right|_{E}: E \rightarrow \phi(E)$ é uma bijeção, temos

$$
\sum_{j \in E} x_{\phi(j)}=\sum_{\phi(j) \in \phi(E)} x_{\phi(j)}=\sum_{i \in \phi(E)} x_{i} .
$$

Assim, tomando os supremos das somas finitas em (1.3.4), obtemos (1.3.3).

Lema 1.3.13. Se $\left(x_{i j}\right)_{(i, j) \in I \times J}$ é uma família de números reais não negativos então

$$
\sum_{i \in I} \sum_{j \in J} x_{i j}=\sum_{(i, j) \in I \times J} x_{i j} .
$$

Demonstração. Seja $H \subset I \times J$ finito. Então existem $F \subset I$ e $G \subset J$ finitos tais que $H \subset F \times G$, e assim

$$
\sum_{(i, j) \in H} x_{i j} \leq \sum_{(i, j) \in F \times G} x_{i j}=\sum_{i \in F} \sum_{j \in G} x_{i j} \leq \sum_{i \in F} \sum_{j \in J} x_{i j} \leq \sum_{i \in I} \sum_{j \in J} x_{i j} .
$$

Portanto,

$$
\sum_{(i, j) \in I \times J} x_{i j}=\sup _{\substack{H \subset I \times J \\ H \text { finito }}} \sum_{(i, j) \in H} x_{i j} \leq \sum_{i \in I} \sum_{j \in J} x_{i j} .
$$

Suponhamos que exista $i \in I$ tal que $\sum_{j \in J} x_{i j}=+\infty$. Então

$$
\sum_{(i, j) \in I \times J} x_{i j} \geq \sum_{j \in J} x_{i j}=+\infty,
$$

o que implica

$$
\sum_{i \in I} \sum_{j \in J} x_{i j} \leq \sum_{(i, j) \in I \times J} x_{i j}
$$


Por outro lado, se $\sum_{j \in J} x_{i j}<+\infty$ para todo $i \in I$ então, dado $\varepsilon>0$, para cada $i \in I$ existe $G_{i} \subset J$ finito tal que $\sum_{j \in G_{i}} x_{i j}>\sum_{j \in J} x_{i j}-\varepsilon$. Assim, para todo $F \subset I$ finito temos:

$$
\begin{gathered}
\sum_{(i, j) \in I \times J} x_{i j} \geq \sum_{(i, j) \in F \times G_{i}} x_{i j}=\sum_{i \in F} \sum_{j \in G_{i}} x_{i j}>\sum_{i \in F} \sum_{j \in J} x_{i j}-\varepsilon|F|, \\
\text { i.e., } \quad \sum_{i \in F} \sum_{j \in J} x_{i j}<\sum_{(i, j) \in I \times J} x_{i j}+\varepsilon|F|, \text { para todo } \varepsilon>0 .
\end{gathered}
$$

Logo, $\sum_{i \in F} \sum_{j \in J} x_{i j} \leq \sum_{(i, j) \in I \times J} x_{i j}$ para todo $F \subset I$ finito, e portanto

$$
\sum_{i \in I} \sum_{j \in J} x_{i j}=\sup _{\substack{F \subset I \\ F \text { finito }}}\left(\sum_{i \in F} \sum_{j \in J} x_{i j}\right) \leq \sum_{(i, j) \in I \times J} x_{i j} .
$$

Corolário 1.3.14. Se $\left(x_{i j}\right)_{(i, j) \in I \times J}$ é uma família de números reais não negativos então

$$
\sum_{i \in I} \sum_{j \in J} x_{i j}=\sum_{j \in J} \sum_{I \in I} x_{i j}
$$

Demonstração. Segue imediatamente da Observação 1.3.12 e do Lema 1.3.13; basta tomar a bijeção $\phi: J \times I \ni(j, i) \longmapsto(i, j) \in I \times J$.

ObSERVAÇÃo 1.3.15. Se $\left(x_{i}\right)_{i \in I}$ é uma família de números reais não negativos tal que $\sum_{i \in I} x_{i}<+\infty$, então o conjunto $J=\left\{i \in I: x_{i} \neq 0\right\}$ é enumerável. Com efeito, para cada número natural $n \geq 1$, o conjunto $J_{n}=\left\{i \in I: x_{i} \geq \frac{1}{n}\right\}$ é finito, senão teríamos

$$
\sum_{i \in I} x_{i} \geq \sum_{i \in J_{n}} x_{i} \geq \sum_{i \in J_{n}} \frac{1}{n}=+\infty .
$$

Como $J=\bigcup_{n \geq 1} J_{n}$, segue que $J$ é enumerável.

Proposição 1.3.16. Uma família $\left(x_{i}\right)_{i \in I}$ de números reais não negativos é somável se e somente se (1.3.2) é finito, e nesse caso (1.3.1) e (1.3.2) são iguais.

DemonstraÇÃo. Suponhamos que

$$
\alpha=\sup \left\{\sum_{i \in F} x_{i}: F \subset I, F \text { finito }\right\}<+\infty .
$$

Dado $\varepsilon>0$, existe $F^{\prime} \subset I$ finito tal que

$$
\alpha-\varepsilon<\sum_{i \in F^{\prime}} x_{i}<\alpha+\varepsilon
$$


Seja $F \subset I$ finito tal que $F \supset F^{\prime}$. Então

$$
\alpha-\varepsilon<\sum_{i \in F^{\prime}} x_{i} \leq \sum_{i \in F} x_{i} \leq \sup _{\substack{F \subset I \\ F \text { finito }}} \sum_{i \in F} x_{i}=\alpha \leq \alpha+\varepsilon,
$$

ou seja, $\left|\alpha-\sum_{i \in F} x_{i}\right|<\varepsilon$. Logo $\left(x_{i}\right)_{i \in I}$ é somável e $\alpha=\sum_{i \in I} x_{i}$. Reciprocamente, suponhamos que $\left(x_{i}\right)_{i \in I}$ seja uma família somável. Então existe $s \in \mathbb{R}$ tal que, dado $\varepsilon>0$, existe $F_{\varepsilon} \subset I$ finito tal que $s-\varepsilon<\sum_{i \in F} x_{i}<s+\varepsilon$, para todo $F \subset I$ finito com $F \supset F_{\varepsilon}$. Seja $F^{\prime} \subset I$ finito arbitrário. Então

$$
\sum_{i \in F^{\prime}} x_{i} \leq \sum_{i \in F^{\prime} \cup F_{\varepsilon}} x_{i}<s+\varepsilon
$$

e portanto

$$
\sup \left\{\sum_{i \in F} x_{i}: F \subset I, F \text { finito }\right\} \leq s+\varepsilon .
$$

Por outro lado, $s-\varepsilon<\sum_{i \in F} x_{i}$, para todo $F \subset I$ finito com $F \supset F_{\varepsilon}$; logo

$$
\begin{aligned}
s-\varepsilon & \leq \sup \left\{\sum_{i \in F} x_{i}: F \subset I, F \text { finito, } F \supset F_{\varepsilon}\right\} \\
& \leq \sup \left\{\sum_{i \in F} x_{i}: F \subset I, F \text { finito }\right\} .
\end{aligned}
$$

Assim, para todo $\varepsilon>0$ temos

$$
s-\varepsilon \leq \sup \left\{\sum_{i \in F} x_{i}: F \subset I, F \text { finito }\right\} \leq s+\varepsilon,
$$

ou seja,

$$
\mid s-\sup \left\{\sum_{i \in F} x_{i}: F \subset I, F \text { finito }\right\} \mid \leq \varepsilon,
$$

para todo $\varepsilon>0 ;$ portanto $\sup \left\{\sum_{i \in F} x_{i}: F \subset I, F\right.$ finito $\}=s<+\infty$

\subsection{Tópicos de Teoria da Medida}

Esta é naturalmente a seção mais extensa desse capítulo. Apresentamos aqui o Teorema de Representação de Riesz para espaços de medida, objeto de estudo desta dissertação, e abordamos todos os tópicos de teoria da medida importantes para uma melhor compreensão das definições e provas dadas a partir do capítulo 2. Muitos resultados aqui colocados foram deslocados dos outros capítulos com a finalidade de tornar as provas apresentadas nesses capítulos mais "limpas", facilitando sua leitura e entendimento. 
OBSERVAÇÃo 1.4.1. Sejam $I$ um intervalo da reta e $a, b \in \mathbb{R}$, com $a \leq b$. O comprimento $l(I)$ do intervalo $I$ é igual a $b-a$ se $I$ for do tipo $[a, b],[a, b[$, ]$a, b]$ ou $] a, b[$, e é igual a $+\infty$ se $I$ for do tipo $]-\infty, a],]-\infty, a[] a,,+\infty[$, $[a,+\infty[$ ou $]-\infty,+\infty[$.

DeFiniÇÃo 1.4.2. A medida exterior de Lebesgue em $\mathbb{R}$, denotada por $\mathfrak{m}^{*}$, é definida como a aplicação:

$$
\begin{aligned}
\mathfrak{m}^{*}: \mathcal{P}(\mathbb{R}) & \longrightarrow[0,+\infty] \\
A & \longmapsto \mathfrak{m}^{*}(A)=\inf \mathcal{I}_{A},
\end{aligned}
$$

$\mathcal{I}_{A}=\left\{\sum_{n \geq 1} l\left(I_{n}\right):\left(I_{n}\right)_{n \geq 1}\right.$ seqüência de intervalos abertos com $\left.A \subset \bigcup_{n \geq 1} I_{n}\right\}$

DEFINIÇÃo 1.4.3. Um conjunto $E \subset \mathbb{R}$ é chamado Lebesgue mensurável (ou $\mathfrak{m}^{*}$-mensurável) se para todo $A \subset \mathbb{R}$ temos

$$
\mathfrak{m}^{*}(A)=\mathfrak{m}^{*}(A \cap E)+\mathfrak{m}^{*}\left(A \cap E^{c}\right) .
$$

Denotamos por $\mathcal{L}$ a coleção dos conjuntos Lebesgue mensuráveis de $\mathbb{R}$.

DefiniÇÃo 1.4.4. A aplicação $\mathfrak{m}=\left.\mathfrak{m}^{*}\right|_{\mathcal{L}}: \mathcal{L} \rightarrow[0,+\infty]$, restrição da medida exterior de Lebesgue aos conjuntos Lebesgue mensuráveis de $\mathbb{R}$, é chamada medida de Lebesgue na reta.

DefiniçÃo 1.4.5. Seja $X$ um conjunto. Uma $\sigma$-álgebra de partes de $X$ é um subconjunto não vazio $\mathcal{A} \subset \mathcal{P}(X)$ satisfazendo as seguintes condições:

(a) se $A \in \mathcal{A}$ então $A^{c} \in \mathcal{A}$;

(b) se $\left(A_{n}\right)_{n \geq 1}$ é uma seqüência de elementos de $\mathcal{A}$ então $\bigcup_{n \geq 1} A_{n} \in \mathcal{A}$.

Em outras palavras, uma $\sigma$-álgebra de partes de $X$ é uma coleção não vazia de subconjuntos de $X$ fechada para complementação e união enumerável (infinita). Observamos que para toda $\sigma$-álgebra $\mathcal{A}$ de partes de $X$, se $A, B \in \mathcal{A}$, então $A \cup B \in \mathcal{A}$ (basta tomar a seqüência $\left(A_{n}\right)_{n \geq 1}$ na qual $A_{1}=A$ e $A_{n}=B$ para todo $n \geq 2$ ), e portanto uma $\sigma$-álgebra também é fechada para união finita. Como $\mathcal{A} \neq \emptyset$, existe um elemento $A \in \mathcal{A}$, e portanto $X=A \cup A^{c} \in \mathcal{A}$ e $\emptyset=X^{c} \in \mathcal{A}$. Observamos por fim que uma $\sigma$-álgebra também é fechada para diferença e intersecção enumerável.

Exemplo 1.4.6. A coleção $\mathcal{L}$ dos conjuntos Lebesgue mensuráveis de $\mathbb{R}$ é uma $\sigma$-álgebra de partes de $\mathbb{R}$. 
OBSERVAÇÃo 1.4.7. Se $\left(\mathcal{A}_{i}\right)_{\in I}$ é uma família não vazia de $\sigma$-álgebras de partes de um conjunto $X$ então $\bigcap_{i \in I} \mathcal{A}_{i}$ também é uma $\sigma$-álgebra de subconjuntos de $X$. Com efeito, $\bigcap_{i \in I} \mathcal{A}_{i} \neq \emptyset$, pois $\emptyset \in A_{i}$ para todo $i \in I$. Se $A \in \bigcap_{i \in I} \mathcal{A}_{i}$ então $A \in A_{i}$ (e portanto $A^{c} \in A_{i}$ ) para cada $i \in I$, $\operatorname{logo} A^{c} \in \bigcap_{i \in I} \mathcal{A}_{i}$. Por fim, se $\left(A_{n}\right)_{n \geq 1}$ é uma seqüência de elementos de $\bigcap_{i \in I} \mathcal{A}_{i}$ então $\left(A_{n}\right)_{n \geq 1}$ é uma seqüência em $A_{i}$, para cada $i \in I$; portanto $\bigcup_{n \geq 1} A_{n} \in A_{i}$ para todo $i \in I$, ou seja, $\bigcup_{n \geq 1} A_{n} \in \bigcap_{i \in I} \mathcal{A}_{i}$.

EXemplo 1.4.8. Se $X$ é um conjunto não enumerável, então a coleção

$$
\mathcal{A}=\left\{A \subset X: A \text { é enumerável ou } A^{c} \text { é enumerável }\right\}
$$

é uma $\sigma$-álgebra de partes de $X$. Com efeito, temos que $\mathcal{A} \neq \emptyset$, pois o conjunto vazio $\emptyset$ é enumerável; se $A \in \mathcal{A}$ então $A$ é enumerável ou $A^{c}$ é enumerável, ou seja, $\left(A^{c}\right)^{c}$ é enumerável ou $A^{c}$ é enumerável, o que implica que $A^{c} \in \mathcal{A}$. Além disso, se $\left(A_{n}\right)_{n \geq 1}$ é uma seqüência de elementos de $\mathcal{A}$, então $\bigcup_{n \geq 1} A_{n}$ é enumerável se $A_{n}$ é enumerável para todo $n \geq 1$. Se, por outro lado, existir $k \geq 1$ tal que $\left(A_{k}\right)^{c}$ seja enumerável, então $\left(\bigcup_{n \geq 1} A_{n}\right)^{c}$ é enumerável, uma vez que $\left(\bigcup_{n \geq 1} A_{n}\right)^{c} \subset\left(A_{k}\right)^{c}$. Portanto $\bigcup_{n \geq 1} A_{n} \in \mathcal{A}$, o que finaliza a justificativa de que $\mathcal{A}$ é uma $\sigma$-álgebra de subconjuntos de $X$.

Proposição 1.4.9. Seja $f: X \rightarrow Y$ uma função. Se $\mathcal{A}$ é uma $\sigma$-álgebra em $Y$ então a coleção $f^{*} \mathcal{A}=\left\{f^{-1}(A): A \in \mathcal{A}\right\}$ é uma $\sigma$-álgebra em $X$ (chamada de $\sigma$-álgebra induzida por $f(e \mathcal{A})$ em $X)$.

Demonstração. Como $X=f^{-1}(Y)$ e $Y \in \mathcal{A}$ temos $X \in f^{*} \mathcal{A}$, e portanto $f^{*} \mathcal{A} \neq \emptyset$. Se $A \in f^{*} \mathcal{A}$ então existe $B \in \mathcal{A}$ tal que $A=f^{-1}(B)$; $\operatorname{logo} A^{c}=\left(f^{-1}(B)\right)^{c}=f^{-1}\left(B^{c}\right)$ e $B^{c} \in \mathcal{A}$, ou seja, $A^{c} \in f^{*} \mathcal{A}$. Se $\left(A_{n}\right)_{n \geq 1}$ é uma seqüência de elementos de $f^{*} \mathcal{A}$ então, para cada $n \geq 1$, existe $B_{n} \in \mathcal{A}$ tal que $A_{n}=f^{-1}\left(B_{n}\right)$. Assim,

$$
\bigcup_{n \geq 1} A_{n}=\bigcup_{n \geq 1} f^{-1}\left(B_{n}\right)=f^{-1}\left(\bigcup_{n \geq 1} B_{n}\right) \text { e } \bigcup_{n \geq 1} B_{n} \in \mathcal{A}
$$

isto é, $\bigcup_{n \geq 1} A_{n} \in f_{*} \mathcal{A}$. Portanto $f^{*} \mathcal{A}$ é uma $\sigma$-álgebra de partes de $X$.

Proposição 1.4.10. Seja $f: X \rightarrow Y$ uma função. Se $\mathcal{A}$ é uma $\sigma$-álgebra em $X$ então a coleção $f_{*} \mathcal{A}=\left\{A \subset Y: f^{-1}(A) \in \mathcal{A}\right\}$ é uma $\sigma$-álgebra em $Y$ (chamada de $\sigma$-álgebra co-induzida por $f$ (e $\mathcal{A})$ em $Y$ ). 
Demonstração. Uma vez que $f^{-1}(Y)=X \in \mathcal{A}$, temos $Y \in f_{*} \mathcal{A}$, e portanto $f_{*} \mathcal{A} \neq \emptyset$. Se $A \in f_{*} \mathcal{A}$ então $f^{-1}(A) \in \mathcal{A}$, o que implica

$$
f^{-1}\left(A^{c}\right)=\left(f^{-1}(A)\right)^{c} \in \mathcal{A},
$$

i.e., $A^{c} \in f_{*} \mathcal{A}$. Se $\left(A_{n}\right)_{n \geq 1}$ é uma seqüência de elementos de $f_{*} \mathcal{A}$ temos

$$
f^{-1}\left(\bigcup_{n \geq 1} A_{n}\right)=\left(\bigcup_{n \geq 1} f^{-1}\left(A_{n}\right)\right) \in \mathcal{A}
$$

pois $f^{-1}\left(A_{n}\right) \in \mathcal{A}$ para cada $n \geq 1$, e assim $\bigcup_{n \geq 1} A_{n} \in f_{*} \mathcal{A}$. Portanto $f_{*} \mathcal{A}$ é uma $\sigma$-álgebra de partes de $Y$.

Proposição 1.4.11. Seja $\left(f_{i}: X_{i} \rightarrow Y\right)_{i \in I}$ uma família de funções. Se $\mathcal{A}_{i}$ é uma $\sigma$-álgebra em $X_{i}$, para cada $i \in I$, então a coleção

$$
\mathcal{A}=\left\{A \subset Y: f_{i}^{-1}(A) \in \mathcal{A}_{i}, \text { para todo } i \in I\right\}
$$

é uma $\sigma$-álgebra de partes de $Y$ (chamada de $\sigma$-álgebra co-induzida em $Y$ pela familia de funções $\left(f_{i}\right)_{i \in I}$ (e pela família de $\sigma$-álgebras $\left(\mathcal{A}_{i}\right)_{i \in I}$ ).

Demonstração. Decorre imediatamente da Observação 1.4.7 e da Proposição 1.4.10, uma vez que $\mathcal{A}=\bigcap_{i \in I} f_{i_{*}} \mathcal{A}_{i}$.

Proposição 1.4.12. Sejam $X$ um conjunto, $\mathcal{A}$ uma $\sigma$-álgebra de partes de $X$ e $\left(A_{n}\right)_{n \geq 1}$ uma seqüência de elementos de $\mathcal{A}$. Seja $\left(B_{n}\right)_{n \geq 1}$ a seqüência dada por $B_{1}=A_{1}$ e, para todo $n \geq 2$,

$$
B_{n}=A_{n} \backslash \bigcup_{k=1}^{n-1} A_{k}
$$

Então $B_{n} \in \mathcal{A}$ para todo $n \geq 1$, os conjuntos $B_{n}$ são dois a dois disjuntos $e$

$$
\bigcup_{n \geq 1} B_{n}=\bigcup_{n \geq 1} A_{n}
$$

Demonstração. $B_{1}$ obviamente está em $\mathcal{A}$, e para $n \geq 2$ também temos $B_{n} \in \mathcal{A}$, já que $\mathcal{A}$ é fechada para união finita e diferença. Sejam $i, j \geq 1$ com $i \neq j$, digamos $i<j$. Então

$$
B_{i} \cap B_{j}=\left(A_{i} \backslash \bigcup_{k=1}^{i-1} A_{k}\right) \cap\left(A_{j} \backslash \bigcup_{k=1}^{j-1} A_{k}\right) \subset A_{i} \cap\left(A_{j} \backslash A_{i}\right)=\emptyset .
$$


Resta mostrar que $\bigcup_{n \geq 1} B_{n}=\bigcup_{n \geq 1} A_{n}$. Para isso, mostraremos antes, por indução sobre $n$, que $\bigcup_{k=1}^{n} B_{k}=\bigcup_{k=1}^{n} A_{k}$ para todo $n \geq 1$. Com efeito, $B_{1}=A_{1}$ e admitindo a igualdade válida para todo $p<n$, temos:

$$
\begin{aligned}
\bigcup_{k=1}^{n} B_{k} & =\left(\bigcup_{k=1}^{n-1} B_{k}\right) \cup B_{n} \\
& =\left(\bigcup_{k=1}^{n-1} A_{k}\right) \cup\left(A_{n} \backslash \bigcup_{k=1}^{n-1} A_{k}\right)=\left(\bigcup_{k=1}^{n-1} A_{k}\right) \cup A_{n}=\bigcup_{k=1}^{n} A_{k} .
\end{aligned}
$$

Portanto $\bigcup_{k=1}^{n} B_{k}=\bigcup_{k=1}^{n} A_{k}$, para todo $n \geq 1$. Como $B_{n} \subset A_{n}$ para todo $n \geq 1$, segue que

$$
\bigcup_{n \geq 1} B_{n} \subset \bigcup_{n \geq 1} A_{n}
$$

Por outro lado, se $x \in \bigcup_{n \geq 1} A_{n}$ então $x \in A_{n_{o}}$ para algum $n_{o} \geq 1$; logo

$$
x \in \bigcup_{k=1}^{n_{o}} A_{k}=\bigcup_{k=1}^{n_{o}} B_{k} \subset \bigcup_{n \geq 1} B_{n}, \quad \text { ou seja, } \bigcup_{n \geq 1} A_{n} \subset \bigcup_{n \geq 1} B_{n} .
$$

Dado um subconjunto $C \subset \mathcal{P}(X)$, a $\sigma$-álgebra obtida pela intersecção de todas as $\sigma$-álgebras que contêm $C$ é chamada de $\sigma$-álgebra gerada por $C$, e é denotada por $[C]$ ou $\sigma[C]$; dizemos também que $C$ é um conjunto de geradores para $[C]$. Observe que $[C]$ é a menor $\sigma$-álgebra de partes de $X$ contendo $C$, ou seja, se $\mathcal{A}$ é uma $\sigma$-álgebra de subconjuntos de $X$ e $C \subset \mathcal{A}$ então $[C] \subset \mathcal{A}$.

A $\sigma$-álgebra de partes de $\mathbb{R}$ gerada pela coleção de todos os subconjuntos abertos de $\mathbb{R}$ é chamada de $\sigma$-álgebra de Borel de $\mathbb{R}$. Denotamos por $\mathcal{B}(\mathbb{R})$ a $\sigma$-álgebra de Borel, e seus elementos são chamados de conjuntos boreleanos de $\mathbb{R}$. Como todo subconjunto aberto de $\mathbb{R}$ é Lebesgue mensurável temos $\mathcal{B}(\mathbb{R}) \subset \mathcal{L}$. É possível mostrar que $\{[a,+\infty[: a \in \mathbb{R}\},\{] a,+\infty[: a \in \mathbb{R}\}$, \{]$-\infty, a]: a \in \mathbb{R}\}$ e \{]$-\infty, a[: a \in \mathbb{R}\}$ são conjuntos de geradores para $\mathcal{B}(\mathbb{R})$. Lembramos ainda, que a coleção $\mathcal{B}(\overline{\mathbb{R}})$ dos boreleanos da reta estendida é a $\sigma$-álgebra gerada pelo conjunto $\{[a,+\infty]: a \in \mathbb{R}\}$, ou equivalentemente, os boreleanos de $\overline{\mathbb{R}}$ são os subconjuntos $A \subset \overline{\mathbb{R}}$ tais que $A \cap \mathbb{R} \in \mathcal{B}(\mathbb{R})$.

DefiniÇÃo 1.4.13. Um espaço mensurável é um par $(X, \mathcal{A})$, no qual $X$ é um conjunto e $\mathcal{A}$ é uma $\sigma$-álgebra de partes de $X$. Os elementos de 
$\mathcal{A}$ são chamados de conjuntos mensuráveis (ou $\mathcal{A}$-mensuráveis, ou ainda, mensuráveis com respeito a $\mathcal{A}$ ) de $X$.

DefiniçÃo 1.4.14. Seja $(X, \mathcal{A})$ um espaço mensurável. Uma medida em $(X, \mathcal{A})$ (ou em $\mathcal{A}$, ou em $X$ ) é uma função $\mu: \mathcal{A} \rightarrow[0,+\infty]$, tal que as seguintes condições são satisfeitas:

(i) $\mu(\emptyset)=0$;

(ii) ( $\sigma$-aditividade) se $\left(A_{n}\right)_{n \geq 1}$ é uma seqüência de elementos de $\mathcal{A}$, dois a dois disjuntos, então

$$
\mu\left(\bigcup_{n \geq 1} A_{n}\right)=\sum_{n \geq 1} \mu\left(A_{n}\right) .
$$

Proposição 1.4.15. Seja $\mu: \mathcal{A} \rightarrow[0,+\infty]$ uma medida em um espaço mensurável $(X, \mathcal{A})$. Então:

(a) Se $A, B \in \mathcal{A}$ e $A \cap B=\emptyset$ então $\mu(A \cup B)=\mu(A)+\mu(B)$.

(b) Se $A$ e $B$ são elementos de $\mathcal{A}$ com $A \subset B$ então $\mu(A) \leq \mu(B)$; além disso, se $\mu(A)<+\infty$ então $\mu(B \backslash A)=\mu(B)-\mu(A)$.

(c) $S e\left(A_{n}\right)_{n \geq 1}$ é uma seqüência de conjuntos $\mathcal{A}$-mensuráveis então

$$
\mu\left(\bigcup_{n \geq 1} A_{n}\right) \leq \sum_{n \geq 1} \mu\left(A_{n}\right) .
$$

DEMONSTRAÇÃO.

- Prova de (a).

Decorre imediatamente da Definição 1.4 .14 ; basta tomar a seqüência $\left(A_{n}\right)_{n \geq 1}$ na qual $A_{1}=A, A_{2}=B$ e $A_{n}=\emptyset$ para todo $n>2$.

- Prova de (b).

Se $A \subset B$ então $B=A \cup(B \backslash A)$ e $A \cap(B \backslash A)=\emptyset$, e portanto

$$
\mu(B)=\mu(A)+\mu(B \backslash A) .
$$

Como $\mu(B \backslash A) \geq 0$, temos $\mu(A) \leq \mu(B)$; além disso, se $\mu(A)<+\infty$ então podemos subtrair $\mu(A)$ de ambos os lados da igualdade $(*)$, e assim obtemos $\mu(B \backslash A)=\mu(B)-\mu(A)$.

- Prova de (c). 
Se $\left(A_{n}\right)_{n \geq 1}$ é uma seqüência de conjuntos $\mathcal{A}$-mensuráveis então, pela Proposição 1.4.12, existe uma seqüência $\left(B_{n}\right)_{n \geq 1}$ de conjuntos mensuráveis, dois a dois disjuntos, tais que $A_{n} \subset B_{n}$ para todo $n \geq 1 \mathrm{e}$ $\bigcup_{n \geq 1} B_{n}=\bigcup_{n \geq 1} A_{n}$. Assim,

$$
\mu\left(\bigcup_{n \geq 1} A_{n}\right)=\mu\left(\bigcup_{n \geq 1} B_{n}\right)=\sum_{n \geq 1} \mu\left(B_{n}\right) \leq \sum_{n \geq 1} \mu\left(A_{n}\right),
$$

uma vez que $\mu\left(A_{n}\right) \leq \mu\left(B_{n}\right)$ para cada $n \geq 1$.

ObSERVAÇÃo 1.4.16. Se $\mu(X)<+\infty$, dizemos que $\mu$ é uma medida finita, e $\mu$ é chamada de $\sigma$-finita se existe uma seqüência de conjuntos mensuráveis $\left(E_{n}\right)_{n \geq 1} \operatorname{com} \mu\left(E_{n}\right)<+\infty$ para todo $n \geq 1$ e $X=\bigcup_{n \geq 1} E_{n}$.

DeFiniÇão 1.4.17. Um espaço de medida é uma terna $(X, \mathcal{A}, \mu)$, na qual $(X, \mathcal{A})$ é um espaço mensurável e $\mu$ é uma medida em $\mathcal{A}$.

ObSERVAÇÃo 1.4.18. Um espaço de medida $(X, \mathcal{A}, \mu)$ é chamado de $\sigma$-finito se $\mu$ é uma medida $\sigma$-finita. Um subconjunto $A \subset X$ é $\sigma$-finito para $\mu$ (ou apenas $\sigma$-finito) se existe uma seqüência de elementos de $\mathcal{A}$ de medida $\mu$ finita, tal que $A=\bigcup_{n \geq 1} A_{n}$.

EXEMPLO 1.4.19. O par $(\mathbb{R}, \mathcal{L})$ é um espaço mensurável e a medida de Lebesgue $\mathfrak{m}$ é uma medida em $\mathcal{L}$. Assim, $(\mathbb{R}, \mathcal{L}, \mathfrak{m})$ é um espaço de medida; na verdade $(\mathbb{R}, \mathcal{L}, \mathfrak{m})$ é um espaço de medida $\sigma$-finito, pois $\mathbb{R}=\bigcup_{n \geq 1}[-n,+n]$ e $\mathfrak{m}([-n,+n])=2 n$, para todo $n \geq 1$.

Proposição 1.4.20. Sejam $X$ um conjunto e $\mathcal{A}$ uma $\sigma$-álgebra de partes de $X$. Se $\left(\mu_{i}: \mathcal{A} \rightarrow[0,+\infty]\right)_{i \in I}$ é uma família de medidas em $\mathcal{A}$ então $\mu=\sum_{i \in I} \mu_{i}$ é uma medida em $\mathcal{A}$.

Demonstração. Para cada $A \in \mathcal{A}, \mu_{i}(A) \geq 0$ para todo $i \in I$. Logo $\mu(A)=\sum_{i \in I} \mu_{i}(A) \in[0,+\infty]$ para todo $A \in \mathcal{A}$, o que mostra que $\mu$ está bem definida. Claramente temos $\mu(\emptyset)=0$. Seja $\left(A_{n}\right)_{n \geq 1}$ uma seqüência de elementos de $\mathcal{A}$, dois a dois disjuntos. Então, pelo Corolário 1.3.14,

$$
\begin{aligned}
\mu\left(\bigcup_{n \geq 1} A_{n}\right) & =\sum_{i \in I} \mu_{i}\left(\bigcup_{n \geq 1} A_{n}\right) \\
& =\sum_{i \in I} \sum_{n \geq 1} \mu_{i}\left(A_{n}\right)=\sum_{n \geq 1} \sum_{i \in I} \mu_{i}\left(A_{n}\right)=\sum_{n \geq 1} \mu\left(A_{n}\right),
\end{aligned}
$$

ou seja, $\mu$ é $\sigma$-aditiva. Portanto $\mu$ é uma medida em $\mathcal{A}$. 
Corolário 1.4.21. Seja $(X, \mathcal{A})$ é um espaço mensurável. Se $\mu_{1}$ e $\mu_{2}$ são duas medidas em $\mathcal{A}$ então $\mu_{1}+\mu_{2}$ é uma medida em $\mathcal{A}$.

Demonstração. Segue imediatamente da Proposição1.4.20; basta considerar a família de medidas $\left(\mu_{i}: \mathcal{A} \rightarrow[0,+\infty]\right)_{i \in I}$, em que $I=\{1,2\}$.

Exemplo 1.4 .22 (medida de Dirac). Sejam $X$ um conjunto e $x \in X$ fixado. A aplicação $\delta_{x}: \mathcal{P}(X) \rightarrow[0,+\infty]$, definida por

$$
\delta_{x}(E)= \begin{cases}1 & \text { se } x \in E \\ 0 & \text { se } x \notin E\end{cases}
$$

é uma medida em $(X, \mathcal{P}(X))$. Com efeito, $\delta_{x}(\emptyset)=0$, pois $x \notin \emptyset$. Seja $\left(A_{n}\right)_{n \geq 1}$ uma seqüência de subconjuntos de $X$, dois a dois disjuntos. Se $x \in \bigcup_{n \geq 1} A_{n}$ então $\delta_{x}\left(\bigcup_{n \geq 1} A_{n}\right)=1$ e $x \in A_{k}$ para algum $k \geq 1$. Como os conjuntos $A_{n}$ são dois a dois disjuntos temos que $x \notin A_{n}$ para todo $n \neq k$, e assim

$$
\sum_{n \geq 1} \delta_{x}\left(A_{n}\right)=\delta_{x}\left(A_{k}\right)+\sum_{\substack{n \geq 1 \\ n \neq k}} \delta_{x}\left(A_{n}\right)=1+0=1 .
$$

Se $x \notin \bigcup_{n \geq 1} A_{n}$ então $\delta_{x}\left(\bigcup_{n \geq 1} A_{n}\right)=0$ e $x \notin A_{n}$ para todo $n \geq 1$, o que implica $\sum_{n \geq 1} \delta_{x}\left(A_{n}\right)=0$. Em qualquer caso temos

$$
\delta_{x}\left(\bigcup_{n \geq 1} A_{n}\right)=\sum_{n \geq 1} \delta_{x}\left(A_{n}\right)
$$

Exemplo 1.4.23 (medida de contagem). Sejam $X$ um conjunto. A aplicação $\mu: \mathcal{P}(X) \rightarrow[0,+\infty]$, dada por

$$
\mu(E)= \begin{cases}|E| & \text { se } E \text { é finito } \\ +\infty & \text { se } E \text { é infinito }\end{cases}
$$

é uma medida no espaço mensurável $(X, \mathcal{P}(X))$. De fato, para cada subconjunto $E \subset X$ temos

$$
\mu(E)=\sum_{x \in E} \delta_{x}(E)=\sum_{x \in X} \delta_{x}(E), \text { ou seja, } \mu=\sum_{x \in X} \delta_{x} .
$$

Portanto, pela Proposição 1.4.20, $\mu$ é uma medida em $\mathcal{P}(X)$. 
Proposição 1.4.24. Sejam $X$ um conjunto não enumerável e $\mathcal{A} a$ $\sigma$-álgebra consistindo dos subconjuntos de $X$ que são enumeráveis ou têm complementar enumerável ${ }^{3}$. Então a aplicação $\mu: \mathcal{A} \rightarrow[0,+\infty]$, dada por

$$
\mu(A)=\left\{\begin{array}{l}
0 \quad \text { se } A \text { é enumerável } \\
1 \text { se } A^{c} \text { é enumerável }
\end{array}\right.
$$

para todo $A \in \mathcal{A}$, é uma medida em $\mathcal{A}$.

Demonstração. Como o conjunto vazio é enumerável temos $\mu(\emptyset)=0$. Seja $\left(A_{n}\right)_{n \geq 1}$ uma seqüência de elementos de $\mathcal{A}$, dois a dois disjuntos. Se $A_{n}$ é enumerável para todo $n \geq 1$ então $\bigcup_{n \geq 1} A_{n}$ é enumerável e $\mu\left(A_{n}\right)=0$ para todo $n \geq 1$; $\operatorname{logo} \mu\left(\bigcup_{n \geq 1} A_{n}\right)=0=\sum_{n \geq 1} \mu\left(A_{n}\right)$. Suponhamos agora que exista $n_{o} \geq 1$ tal que $A_{n_{o}}^{c}$ seja enumerável. Então $\left(\bigcup_{n \geq 1} A_{n}\right)^{c}$ é enumerável, pois $\left(\bigcup_{n \geq 1} A_{n}\right)^{c} \subset A_{n_{o}}^{c}$, e portanto $\mu\left(\bigcup_{n \geq 1} A_{n}\right)=1$. Por outro lado, visto que os conjuntos $A_{n}$ são dois a dois disjuntos, temos $\left(\bigcup_{n \geq 1} A_{n}\right) \backslash A_{n_{o}} \subset A_{n_{o}}^{c}$; logo, para todo $k \geq 1, k \neq n_{o}$, temos que

$$
\mu\left(A_{k}\right) \leq \mu\left(\left(\bigcup_{n \geq 1} A_{n}\right) \backslash A_{n_{o}}\right) \leq \mu\left(A_{n_{o}}^{c}\right)=0 \text {, ou seja, } \mu\left(A_{k}\right)=0 .
$$

Assim,

$$
\sum_{n \geq 1} \mu\left(A_{n}\right)=\mu\left(A_{n_{o}}\right)+\sum_{\substack{n \geq 1 \\ n \neq n_{o}}} \mu\left(A_{n}\right)=1+0=1,
$$

e conseqüentemente $\mu\left(\bigcup_{n \geq 1} A_{n}\right)=\sum_{n \geq 1} \mu\left(A_{n}\right)$. Em qualquer caso a aplicação $\mu$ é $\sigma$-aditiva, e isso mostra que $\mu$ é uma medida em $\mathcal{A}$.

Proposição 1.4.25. Seja $(X, \mathcal{A}, \mu)$ um espaço de medida.

(a) Se $\left(E_{n}\right)_{n \geq 1}$ é uma seqüência crescente de elementos de $\mathcal{A}$, i.e., $E_{1} \subset E_{2} \subset \cdots \subset E_{n} \subset \cdots$, então

$$
\mu\left(\bigcup_{n \geq 1} E_{n}\right)=\lim _{n \rightarrow+\infty} \mu\left(E_{n}\right) .
$$

(b) Se $\left(F_{n}\right)_{n \geq 1}$ é uma seqüência decrescente de elementos de $\mathcal{A}$, i.e., $F_{1} \supset F_{2} \supset \cdots \supset F_{n} \supset \cdots$, e $\mu\left(F_{1}\right)<+\infty$, então

$$
\mu\left(\bigcap_{n \geq 1} F_{n}\right)=\lim _{n \rightarrow+\infty} \mu\left(F_{n}\right) .
$$

Demonstração. Vide [3].

\footnotetext{
${ }^{3}$ Exemplo 1.4.8.
} 
Se $(X, \mathcal{A})$ é um espaço mensurável e $Y$ é um subconjunto de $X$ então a coleção $\left.\mathcal{A}\right|_{Y}=\{E \cap Y: E \in \mathcal{A}\}$ é uma $\sigma$-álgebra de partes de $Y$. De fato, pela Proposição $1.4 .9,\left.\mathcal{A}\right|_{Y}$ é a $\sigma$-álgebra induzida em $Y$ por $\mathcal{A}$ e pela aplicação inclusão $i: Y \rightarrow X$ :

$$
\left.\mathcal{A}\right|_{Y}=\{E \cap Y: E \in \mathcal{A}\}=\left\{i^{-1}(E): E \in \mathcal{A}\right\}=i^{*} \mathcal{A}
$$

Diremos (apenas) que $\left.\mathcal{A}\right|_{Y}$ é a $\sigma$-álgebra induzida em $Y$ por $\mathcal{A}$. Dizemos também que $\left(Y,\left.\mathcal{A}\right|_{Y}\right)$ é subespaço mensurável do espaço mensurável $(X, \mathcal{A})$. Se $Y \in \mathcal{A}$ então os elementos da $\sigma$-álgebra $\left.\mathcal{A}\right|_{Y}$ são exatamente os elementos de $\mathcal{A}$ que estão contidos em $Y$. Em outras palavras, se $Y$ é $\mathcal{A}$-mensurável então os subconjuntos $\left.\mathcal{A}\right|_{Y}$-mensuráveis do subespaço $Y$ são os subconjuntos mensuráveis de $X$ que estão contidos em $Y$, isto é, $\left.\mathcal{A}\right|_{Y}=\mathcal{A} \cap \mathcal{P}(Y)$. Logo, se $\mu: \mathcal{A} \rightarrow[0,+\infty]$ é uma medida $\operatorname{em~}(X, \mathcal{A})$ então a restrição $\left.\mu\right|_{\left.\mathcal{A}\right|_{Y}}:\left.\mathcal{A}\right|_{Y} \rightarrow[0,+\infty]$ é uma medida em $\left(Y,\left.\mathcal{A}\right|_{Y}\right)$. Assim, podemos concluir que se $(X, \mathcal{A}, \mu)$ é um espaço de medida arbitrário e $Y$ um subconjunto $\mathcal{A}$-mensurável de $X$ então $\left(Y,\left.\mathcal{A}\right|_{Y},\left.\mu\right|_{\left.\mathcal{A}\right|_{Y}}\right)$ é também um espaço de medida, chamado de um subespaço de medida de $(X, \mathcal{A}, \mu)$. Dados um espaço de medida $(X, \mathcal{A}, \mu)$ e um subconjunto $\mathcal{A}$-mensurável $Y$, quando $Y$ for encarado como subespaço de medida, admitiremos $Y$ munido com a $\sigma$-álgebra $\left.\mathcal{A}\right|_{Y}$ e a medida $\left.\mu\right|_{\left.\mathcal{A}\right|_{Y}}$.

DefiniçÃo 1.4.26. Seja $(X, \mathcal{A})$ um espaço mensurável. Uma medida $\mu: \mathcal{A} \rightarrow[0,+\infty]$ é chamada de completa se para todo conjunto mensurável $B \in \mathcal{A} \operatorname{com} \mu(B)=0$, cada subconjunto $A \subset B$ é mensurável.

Em outras palavras, $\mu$ é completa se cada subconjunto de conjunto mensurável de medida nula também é mensurável. Nesse caso, o espaço de medida é dito completo. É possível mostrar que se $(X, \mathcal{A}, \mu)$ é um espaço de medida arbitrário e $\mathcal{N}=\{N \in \mathcal{A}: \mu(N)=0\}$ então a coleção

$$
\overline{\mathcal{A}}=\{A \cup F: A \in \mathcal{A} \text { e } F \subset N \in \mathcal{N}\}
$$

é uma $\sigma$-álgebra de partes de $X$ que contém $\mathcal{A}$. Além disso, existe uma única medida $\bar{\mu}: \overline{\mathcal{A}} \rightarrow[0,+\infty]$ que estende $\mu$ e é completa:

$$
\bar{\mu}(A \cup F)=\mu(A), \quad A \in \mathcal{A} \text { e } F \subset N \text { para algum } N \in \mathcal{N} .
$$


Portanto o espaço de medida $(X, \overline{\mathcal{A}}, \bar{\mu})$ é completo e é chamado de o completamento do espaço $(X, \mathcal{A}, \mu)$. Por exemplo, o completamento do espaço de medida $\left(\mathbb{R}, \mathcal{B}(\mathbb{R}),\left.\mathfrak{m}\right|_{\mathcal{B}(\mathbb{R})}\right)$ é o espaço $(\mathbb{R}, \mathcal{L}, \mathfrak{m})$.

OBSERVAÇÃo 1.4.27. Sejam $(X, \mathcal{A}, \mu)$ um espaço de medida e $p(x)$ uma propriedade relativa aos elementos do conjunto $X$. Dizemos que a propriedade $p(x)$ vale $\mu$-quase sempre (ou abreviadamente, $\mu$-qs) se existe um conjunto mensurável $E \in \mathcal{A} \operatorname{com} \mu(E)=0$, tal que $p(x)$ é válida para todo $x \in E^{c}$.

Definição 1.4.28. Sejam $(X, \mathcal{A})$ e $\left(X^{\prime}, \mathcal{A}^{\prime}\right)$ espaços mensuráveis. Uma função mensurável $f:(X, \mathcal{A}) \rightarrow\left(X^{\prime}, \mathcal{A}^{\prime}\right)$ é uma função $f: X \rightarrow X^{\prime}$ tal que $f^{-1}(E) \in \mathcal{A}$ para todo $E \in \mathcal{A}^{\prime}$.

Ou seja, uma função entre espaços mensuráveis é dita mensurável se a imagem inversa de conjunto mensurável é um conjunto mensurável. Uma função mensurável $f:(X, \mathcal{A}) \rightarrow\left(X^{\prime}, \mathcal{A}^{\prime}\right)$ também é chamada de mensurável em relação a $\mathcal{A}$ e $\mathcal{A}^{\prime}$, ou $\left(\mathcal{A}, \mathcal{A}^{\prime}\right)$-mensurável.

OBSERVAÇÃo 1.4.29. Não havendo confusão quanto às $\sigma$-álgebras $\mathcal{A}$ e $\mathcal{A}^{\prime}$ definidas nos conjuntos $X$ e $X^{\prime}$ respectivamente, falaremos economicamente que $f: X \rightarrow X^{\prime}$ é mensurável para expressar que $f:(X, \mathcal{A}) \rightarrow\left(X^{\prime}, \mathcal{A}^{\prime}\right)$ é mensurável. Sempre que $\mathbb{R}$ (resp., $\overline{\mathbb{R}}$ ) aparecer no contradomínio de uma função, consideraremos $\mathbb{R}$ (resp., $\overline{\mathbb{R}}$ ) munido com a $\sigma$-álgebra $\mathcal{B}(\mathbb{R})$ (resp., $\mathcal{B}(\overline{\mathbb{R}})$ ); se $\mathbb{R}$ aparecer no domínio de uma função, admitiremos que $\mathbb{R}$ está munido com a $\sigma$-álgebra $\mathcal{L}$ dos conjuntos Lebesgue mensuráveis de $\mathbb{R}$. Ou seja, dado um espaço mensurável $(X, \mathcal{A})$, uma função $f: X \rightarrow \mathbb{R}$ é mensurável se $f$ é mensurável em relação a $\mathcal{A}$ e $\mathcal{B}(\mathbb{R})$, e uma função $f: \mathbb{R} \rightarrow X$ é mensurável se $f$ é mensurável em relação a $\mathcal{L}$ e $\mathcal{A}$. No caso de uma função $f: X \rightarrow \mathbb{R}$ ser $(\mathcal{A}, \mathcal{B}(\mathbb{R}))$-mensurável , diremos apenas que $f$ é $\mathcal{A}$-mensurável ou mensurável relativamente a $\mathcal{A}$. Do mesmo modo, $f: X \rightarrow \overline{\mathbb{R}}$ é dita $\mathcal{A}$-mensurável se é $(\mathcal{A}, \mathcal{B}(\overline{\mathbb{R}}))$-mensurável.

Proposição 1.4.30. Sejam $(X, \mathcal{A})$ e $\left(X^{\prime}, \mathcal{A}^{\prime}\right)$ espaços mensuráveis, e seja $\mathcal{C} \subset \mathcal{P}\left(X^{\prime}\right)$ um conjunto de geradores de $\mathcal{A}^{\prime}$, i.e., $\mathcal{A}^{\prime}=[\mathcal{C}]$. Uma função $f: X \rightarrow X^{\prime}$ é mensurável se e somente se $f^{-1}(E) \in \mathcal{A}$ para todo $E \in \mathcal{C}$.

Demonstração. Se $f$ é mensurável então $f^{-1}(E) \in \mathcal{A}$ qualquer que seja $E \in \mathcal{A}^{\prime}$, em particular para todo $E \in \mathcal{C}$. Reciprocamente, suponhamos 
que $f^{-1}(E) \in \mathcal{A}$ para todo $E \in \mathcal{C}$. A coleção $\left\{E \subset X^{\prime}: f^{-1}(E) \in \mathcal{A}\right\}$ é a $\sigma$-álgebra co-induzida por $f$ e contém $\mathcal{C}$, logo contém $[\mathcal{C}]=\mathcal{A}^{\prime}$; portanto $f^{-1}(E) \in \mathcal{A}$ para todo $E \in \mathcal{A}^{\prime}$, o que implica $f$ mensurável.

Corolário 1.4.31. Sejam $(X, \mathcal{A})$ um espaço mensurável e $f: X \rightarrow \mathbb{R}$ uma função. Então são equivalentes as seguintes afirmações:

(a) $f$ é mensurável;

(b) $f^{-1}(U) \in \mathcal{A}$ para todo aberto $U \subset \mathbb{R}$;

(c) $\left.\left.f^{-1}(]-\infty, c\right]\right) \in \mathcal{A}$ para todo $c \in \mathbb{R}$.

DemonstraçÃo. Decorre imediatamente da Observação 1.4 .29 e da Proposição 1.4.30, lembrando que $\mathcal{B}(\mathbb{R})$ é a $\sigma$-álgebra gerada pelos abertos de $\mathbb{R}$, e que \{]$-\infty, c]: c \in \mathbb{R}\}$ é um conjunto de geradores de $\mathcal{B}(\mathbb{R})$.

Observamos que o Corolário 1.4.31 continua válido se trocarmos o intervalo $]-\infty, c]$ por $]-\infty, c[,[c,+\infty[$ ou $] c,+\infty[$.

ObSERVAÇÃo 1.4.32. Sejam $X$ um conjunto arbitrário e $g: X \rightarrow \mathbb{R}$ uma função. A função sinal de $g$ é definida por:

$$
\operatorname{sgn}(g)=\chi_{g^{-1}(] 0,+\infty[)}-\chi_{g^{-1}(]-\infty, 0[)} \cdot
$$

Ou seja, $\operatorname{sgn}(g)(x)$ é igual a 1 se $g(x)>0,0$ se $g(x)=0$, e -1 se $g(x)<0$. Se $\mathcal{A}$ é uma $\sigma$-álgebra de partes de $X$ e se $g$ é $\mathcal{A}$-mensurável então, claramente, $\operatorname{sgn}(g)$ também é $\mathcal{A}$-mensurável.

ObservaÇão 1.4.33. Se $(X, \mathcal{A}),\left(X^{\prime}, \mathcal{A}^{\prime}\right)$ e $\left(X^{\prime \prime}, \mathcal{A}^{\prime \prime}\right)$ são espaços mensuráveis e $f:(X, \mathcal{A}) \rightarrow\left(X^{\prime}, \mathcal{A}^{\prime}\right), g:\left(X^{\prime}, \mathcal{A}^{\prime}\right) \rightarrow\left(X^{\prime \prime}, \mathcal{A}^{\prime \prime}\right)$ são funções mensuráveis, então a composta $g \circ f:(X, \mathcal{A}) \rightarrow\left(X^{\prime \prime}, \mathcal{A}^{\prime \prime}\right)$ também é mensurável. De fato, para cada $E \in \mathcal{A}^{\prime \prime}, g^{-1}(E) \in \mathcal{A}^{\prime}$, pois $g$ é mensurável, e portanto $(g \circ f)^{-1}(E)=f^{-1}\left(g^{-1}(E)\right) \in \mathcal{A}$, uma vez que $f$ é mensurável.

ObservaÇão 1.4.34. Sejam $(X, \mathcal{A})$ e $\left(X^{\prime}, \mathcal{A}^{\prime}\right)$ espaços mensuráveis e $Y$ um subconjunto de $X$. Se $f:(X, \mathcal{A}) \rightarrow\left(X^{\prime}, \mathcal{A}^{\prime}\right)$ é uma função mensurável então a função $\left.f\right|_{Y}:\left(Y,\left.\mathcal{A}\right|_{Y}\right) \rightarrow\left(X^{\prime}, \mathcal{A}^{\prime}\right)$ também é mensurável. Com efeito, a aplicação inclusão $i: Y \rightarrow X$ é mensurável, pois $i^{-1}(E)=\left.E \cap Y \in \mathcal{A}\right|_{Y}$ para todo $E \in \mathcal{A}$ e $\left.f\right|_{Y}=f \circ i$. Portanto, pela Observação 1.4.33, a aplicação $\left.f\right|_{Y}$ é mensurável. 
Proposição 1.4.35. Sejam $(X, \mathcal{A})$ e $\left(X^{\prime}, \mathcal{A}^{\prime}\right)$ espaços mensuráveis, e $\left(X_{n}\right)_{n \geq 1}$ uma seqüência de elementos de $\mathcal{A}$ com $X=\bigcup_{n \geq 1} X_{n}$. Então uma aplicação $f: X \rightarrow X^{\prime}$ é mensurável se somente se $\left.f\right|_{X_{n}}: X_{n} \rightarrow X^{\prime}$ é mensurável para todo $n \geq 1$.

Demonstração. Se $f$ é uma função mensurável então $\left.f\right|_{X_{n}}$ é mensurável para todo $n \geq 1$, pela Observação 1.4.34. Reciprocamente, suponhamos que $\left.f\right|_{X_{n}}$ seja mensurável para todo $n \geq 1$. Como $X_{n} \in \mathcal{A}$ temos que $\left.\mathcal{A}\right|_{X_{n}}=\mathcal{A} \cap \mathcal{P}\left(X_{n}\right)$, e portanto $f^{-1}(E) \cap X_{n}=\left(\left.f\right|_{X_{n}}\right)^{-1}(E) \in \mathcal{A}$ para todo $n \geq 1$ e todo $E \in \mathcal{A}^{\prime}$. Assim,

$$
f^{-1}(E)=f^{-1}(E) \cap\left(\bigcup_{n \geq 1} X_{n}\right)=\bigcup_{n \geq 1}\left(f^{-1}(E) \cap X_{n}\right) \in \mathcal{A},
$$

para cada $E \in \mathcal{A}^{\prime}$, o que implica $f$ mensurável.

ProposiçÃo 1.4.36. Seja $Y$ um subconjunto arbitrário de $\mathbb{R}$. Se uma função $f: Y \rightarrow \mathbb{R}$ é contínua então $f$ é mensurável.

Demonstração. Para todo aberto $U \subset \mathbb{R}$ temos que $f^{-1}(U)$ é aberto relativamente a $Y$, pois $f$ é contínua. Logo existe um aberto $V \subset \mathbb{R}$ tal que $f^{-1}(U)=Y \cap V$. Como $V \in \mathcal{B}(\mathbb{R}) \subset \mathcal{L}$, segue que $\left.f^{-1}(U) \in \mathcal{L}\right|_{Y}$, e portanto $f$ é mensurável.

DeFiniÇÃo 1.4.37. Dado $x \in \overline{\mathbb{R}}$, as partes positiva e negativa de $x$, denotadas respectivamente por $x^{+}$e $x^{-}$, são definidas por

$$
x^{+}=\left\{\begin{array}{ll}
x & \text { se } x \geq 0 \\
0 & \text { se } x<0
\end{array} \quad e \quad x^{-}= \begin{cases}0 & \text { se } x>0 \\
-x & \text { se } x \leq 0 .\end{cases}\right.
$$

Se $f: X \rightarrow \overline{\mathbb{R}}$ é uma função então as partes positiva e negativa de $f$, denotadas respectivamente por $f^{+}$e $f^{-}$, são as aplicações $f^{+}, f^{-}: X \rightarrow \overline{\mathbb{R}}$ dadas por $f^{+}(x)=(f(x))^{+}$e $f^{-}(x)=(f(x))^{-}$, para todo $x \in X$.

Observe que para cada $x \in \overline{\mathbb{R}}$ temos $x=x^{+}-x^{-}$e $|x|=x^{+}+x^{-}$, e portanto $f=f^{+}-f^{-}$e $|f|=f^{+}+f^{-}$para toda função $f: X \rightarrow \overline{\mathbb{R}}$.

Proposição 1.4.38. Sejam $(X, \mathcal{A})$ um espaço mensurável, $f, g: X \rightarrow \mathbb{R}$ duas aplicações e c um número real. Se $f$ e g são mensuráveis então também são mensuráveis as aplicações cf $, f^{2}, f+g, f g,|f|, f^{+} e f^{-}$. 
DemonstraÇÃo. Vide [3].

Proposição 1.4.39. Sejam $(X, \mathcal{A})$ um espaço mensurável e $\left(f_{n}\right)_{n \geq 1}$ uma seqüência de funções mensuráveis, $f_{n}: X \rightarrow \mathbb{R}$ para todo $n \geq 1$. Então as aplicações $\inf _{n \geq 1} f_{n}, \sup _{n \geq 1} f_{n}, \liminf f_{n} e \limsup f_{n}$ também são mensuráveis.

DemonstraÇÃo. Vide [22].

ObservaÇÃo 1.4.40. Seja $(X, \mathcal{A})$ um espaço mensurável. Se $\left(f_{n}\right)_{n \geq 1}$ é uma seqüência de funções mensuráveis, $f_{n}: X \rightarrow \mathbb{R}$ para todo $n \geq 1$, cujo limite é uma função $f: X \rightarrow \mathbb{R}$, então $f$ é mensurável. Com efeito,

$$
f=\lim _{n \rightarrow+\infty} f_{n}=\lim \sup f_{n},
$$

o que implica na mensurabilidade de $f$.

Para duas funções funções $f, g: X \rightarrow \overline{\mathbb{R}}$, o produto $f g: X \rightarrow \overline{\mathbb{R}}$ pode ser sempre definido, e a soma $f+g: X \rightarrow \overline{\mathbb{R}}$ pode ser definida caso ela esteja bem definida, ou seja, se para todo $x \in X$ não ocorrem as situações $(-\infty)+(+\infty)$ e $(+\infty)+(-\infty)$. A Proposição 1.4 .38 continua verdadeira se substituirmos os contradomínios de $f$ e $g$ por $\overline{\mathbb{R}}$, e $f+g$ estiver bem definida. Da mesma forma, se trocarmos por $\overline{\mathbb{R}}$ os contradomínios das aplicações da Proposição 1.4.39 e da Observação 1.4.40, elas continuam válidas.

Proposição 1.4.41. Sejam $(X, \mathcal{A}, \mu)$ um espaço de medida completa e $\left(X^{\prime}, \mathcal{A}^{\prime}\right)$ um espaço mensurável. Se $f: X \rightarrow X^{\prime}$ é uma função mensurável, e $g: X \rightarrow X^{\prime}$ uma função tal que $g=f \mu$-qs, então $g$ também é mensurável.

Demonstração. Se $g=f \mu$-qs então existe $A \in \mathcal{A} \operatorname{com} \mu(A)=0$ tal que $g(x)=f(x)$ para todo $x \in A^{c}$. Seja $E \in \mathcal{A}^{\prime}$ arbitrário. Então $g^{-1}(E)=\left(g^{-1}(E) \cap A^{c}\right) \cup\left(g^{-1}(E) \cap A\right)=\left(f^{-1}(E) \cap A^{c}\right) \cup\left(g^{-1}(E) \cap A\right)$. Como $f$ é mensurável temos que $f^{-1}(E) \in \mathcal{A}$, e uma vez que $\mu$ é completa segue que $g^{-1}(E) \cap A \in \mathcal{A}$. Portanto $g^{-1}(E)$ está em $\mathcal{A}$, o que mostra que $g$ é uma aplicação mensurável.

Lema 1.4.42. Se $(X, \mathcal{A}, \mu)$ é um espaço de medida e $f_{1}, f_{2}, g_{1}, g_{2}: X \rightarrow \mathbb{R}$ são funções mensuráveis tais que $f_{1}=g_{1} \mu$-qs e $f_{2}=g_{2} \mu$-qs, então $f_{1}+f_{2}$ e $g_{1}+g_{2}$ são mensuráveis e $f_{1}+f_{2}=g_{1}+g_{2} \mu$-qs. 
Demonstração. A mensurabilidade de $f_{1}+f_{2}$ e $g_{1}+g_{2}$ decorre imediatamente da Proposição 1.4.38. Se $f_{1}=g_{1} \mu$-qs e $f_{2}=g_{2} \mu$-qs então existem $A_{1}, A_{2} \in \mathcal{A}$, com $\mu\left(A_{1}\right)=0$ e $\mu\left(A_{2}\right)=0$, tais que $f_{1}(x)=f_{2}(x)$ para todo $x \in A_{1}^{c}$ e $g_{1}(x)=g_{2}(x)$ para todo $x \in A_{2}^{c}$. Como $\left(A_{1} \cup A_{2}\right)^{c}=A_{1}^{c} \cap A_{2}^{c}$, para cada $x \in\left(A_{1} \cup A_{2}\right)^{c}$, temos

$$
\left(f_{1}+f_{2}\right)(x)=f_{1}(x)+f_{2}(x)=g_{1}(x)+g_{2}(x)=\left(g_{1}+g_{2}\right)(x) .
$$

Por outro lado, $\mu\left(A_{1} \cup A_{2}\right) \leq \mu\left(A_{1}\right)+\mu\left(A_{2}\right)=0$, i.e., $\mu\left(A_{1} \cup A_{2}\right)=0$. Portanto $f_{1}+f_{2}=g_{1}+g_{2} \mu$-qs.

Corolário 1.4.43. Se $f_{1}, \ldots, f_{n}, g_{1}, \ldots, g_{n}: X \rightarrow \mathbb{R}$ são funções mensuráveis tais que $f_{i}=g_{i} \mu$-qs para cada $i \in\{1, \ldots, n\}$, então as funções $f_{1}+\cdots+f_{n}$ e $g_{1}+\cdots+g_{n}$ são mensuráveis e iguais $\mu-q$ s.

DemonstraÇÃo. Imediata, por indução sobre $n$.

Lema 1.4.44. Seja $(X, \mathcal{A}, \mu)$ um espaço de medida. Sejam $\left(f_{n}\right)_{n \geq 1}$ e $\left(g_{n}\right)_{n \geq 1}$ duas seqüências de funções mensuráveis $f_{n}, g_{n}: X \rightarrow \mathbb{R}$, tais que $f_{n}=g_{n} \mu$-qs para todo $n \geq 1$. Se $f=\limsup f_{n}$ e $g=\limsup g_{n}$ então $f$ e $g$ são mensuráveis e $f=g \mu$-qs.

Demonstração. A mensurabilidade de $f$ e $g$ decorre imediatamente da Proposição 1.4.39. Se $f_{n}=g_{n} \mu$-qs para cada $n \geq 1$ então, para cada $n \geq 1$, existe $A_{n} \in \mathcal{A}$ com $\mu\left(A_{n}\right)=0$ tal que $f_{n}(x)=g_{n}(x)$ para todo $x \in A_{n}^{c}$. O conjunto $A=\bigcup_{n \geq 1} A_{n}$ é mensurável e

$$
\mu(A)=\mu\left(\bigcup_{n \geq 1} A_{n}\right) \leq \sum_{n \geq 1} \mu\left(A_{n}\right)=0, \text { i.e., } \mu(A)=0 .
$$

Por outro lado, para cada $x \in A^{c}=\bigcap_{n \geq 1} A_{n}^{c}$, temos $f_{n}(x)=g_{n}(x)$ para todo $n \geq 1$. Assim, para todo $x \in A^{c}$,

$$
f(x)=\lim \sup f_{n}(x)=\lim \sup g_{n}(x)=g(x),
$$

e portanto $f$ e $g$ são iguais $\mu$-qs.

DefiniÇão 1.4.45. Sejam $(X, \mathcal{A}, \mu)$ e $\left(X^{\prime}, \mathcal{A}^{\prime}, \mu^{\prime}\right)$ espaços de medida. Dizemos que uma função $\phi: X \rightarrow X^{\prime}$ preserva medida se $\phi$ é mensurável e se $\mu^{\prime}(A)=\mu\left(\phi^{-1}(A)\right)$ para todo $A \in \mathcal{A}^{\prime}$. 
Proposição 1.4.46. Sejam $(X, \mathcal{A}, \mu)$ um espaço de medida, $\left(X^{\prime}, \mathcal{A}^{\prime}\right)$ um espaço mensurável e $\phi: X \rightarrow X^{\prime}$ uma aplicação mensurável. Então:

(a) a aplicação $\phi_{*} \mu: \mathcal{A}^{\prime} \rightarrow[0,+\infty]$, definida por

$$
\left(\phi_{*} \mu\right)(A)=\mu\left(\phi^{-1}(A)\right),
$$

para todo $A \in \mathcal{A}^{\prime}$, é uma medida em $\mathcal{A}^{\prime}$;

(b) se $\mu^{\prime}$ é uma medida em $\mathcal{A}^{\prime}$ então $\phi:(X, \mathcal{A}, \mu) \rightarrow\left(X^{\prime}, \mathcal{A}^{\prime}, \mu^{\prime}\right)$ preserva medida se e somente se $\phi_{*} \mu=\mu^{\prime}$.

$\phi_{*} \mu$ é chamada de medida co-induzida por $\phi$ e $\mu$ em $\mathcal{A}^{\prime}$.

\section{DemonstraÇÃo.}

- Prova de (a).

$\phi_{*} \mu$ está bem definida: como $\phi$ é uma aplicação mensurável, temos que $\phi^{-1}(A) \in \mathcal{A}$ para todo $A \in \mathcal{A}^{\prime}$, e portanto

$$
\left(\phi_{*} \mu\right)(A)=\mu\left(\phi^{-1}(A)\right) \in[0,+\infty] .
$$

É fácil ver que $\left(\phi_{*} \mu\right)(\emptyset)=0$. Se $\left(A_{n}\right)_{n \geq 1}$ é uma seqüência de elementos de $\mathcal{A}^{\prime}$, dois a dois disjuntos, então $\left(\phi^{-1}\left(A_{n}\right)\right)_{n \geq 1}$ é uma seqüência de elementos de $\mathcal{A}$, dois a dois disjuntos. Assim,

$$
\begin{aligned}
& \left(\phi_{*} \mu\right)\left(\bigcup_{n \geq 1} A_{n}\right)=\mu\left(\phi^{-1}\left(\bigcup_{n \geq 1} A_{n}\right)\right) \\
& \quad=\mu\left(\bigcup_{n \geq 1} \phi^{-1}\left(A_{n}\right)\right)=\sum_{n \geq 1} \mu\left(\phi^{-1}\left(A_{n}\right)\right)=\sum_{n \geq 1}\left(\phi_{*} \mu\right)\left(A_{n}\right) .
\end{aligned}
$$

Portanto $\phi_{*} \mu$ é uma medida em $\mathcal{A}^{\prime}$.

- Prova de (b).

$\phi$ preserva medida se e somente se $\mu^{\prime}(A)=\mu\left(\phi^{-1}(A)\right)=\left(\phi_{*} \mu\right)(A)$ para todo $A \in \mathcal{A}^{\prime}$, ou seja, se e somente se $\mu^{\prime}=\phi_{*} \mu$.

Dados um espaço de medida $(X, \mathcal{A}, \mu)$, um conjunto $X^{\prime}$ e uma aplicação $\phi: X \rightarrow X^{\prime}$, pela Proposição 1.4.10, o conjunto

$$
\phi_{*} \mathcal{A}=\left\{A \subset X^{\prime}: \phi^{-1}(A) \in \mathcal{A}\right\}
$$

é uma $\sigma$-álgebra de partes de $X^{\prime}$ chamada de $\sigma$-álgebra co-induzida por $\phi$ e $\mathcal{A}$ em $X^{\prime}$. Observe que $\phi_{*} \mathcal{A}$ é a "maior" $\sigma$-álgebra de subconjuntos 
de $X^{\prime}$ que torna $\phi$ mensurável; em particular, se $\mathcal{A}^{\prime}$ é uma $\sigma$-álgebra de subconjuntos de $X^{\prime}$ contida em $\phi_{*} \mathcal{A}$ então a aplicação $\phi:(X, \mathcal{A}) \rightarrow\left(X^{\prime}, \mathcal{A}^{\prime}\right)$ é mensurável. Ainda, se $\mu^{\prime}$ é uma medida em $\left(X^{\prime}, \mathcal{A}^{\prime}\right)$ tal que $\mathcal{A}^{\prime} \subset \phi_{*} \mathcal{A}$ e $\mu^{\prime}=\phi_{*} \mu$ então, pelo item (b) da Proposição 1.4.46, $\phi: X \rightarrow X^{\prime}$ é uma aplicação que preserva medida.

Definição 1.4.47. Sejam $(X, \mathcal{A}, \mu)$ e $\left(X^{\prime}, \mathcal{A}^{\prime}, \mu^{\prime}\right)$ espaços de medida. Uma aplicação $\phi: X \rightarrow X^{\prime}$ que preserva medida é chamada de aplicação quociente se $\mathcal{A}^{\prime}=\phi_{*} \mathcal{A}$. Uma função $\phi: X \rightarrow X^{\prime}$ é um isomorfismo se é uma aplicação quociente bijetora.

Proposição 1.4.48. Sejam $(X, \mathcal{A}, \mu)$ e $\left(X^{\prime}, \mathcal{A}^{\prime}, \mu^{\prime}\right)$ espaços de medida. Uma aplicação $\phi: X \rightarrow X^{\prime}$ é um isomorfismo se e somente se é uma bijeção tal que, para todo $A \subset X, \phi(A) \in \mathcal{A}^{\prime}$ se e somente se $A \in \mathcal{A}$, e nesse caso, $\mu^{\prime}(\phi(A))=\mu(A)$

Demonstração. Se $\phi$ é um isomorfismo então é uma bijeção tal que $\mathcal{A}^{\prime}=\phi_{*} \mathcal{A}$ e $\mu^{\prime}(A)=\mu\left(\phi^{-1}(A)\right)$ para cada $A \in \mathcal{A}^{\prime}$. Assim, se $\phi(A) \in \mathcal{A}^{\prime}$ então $\phi^{-1}(\phi(A))=A \in \mathcal{A}$, e reciprocamente, se $A \in \mathcal{A}$ então $\phi(A) \subset X^{\prime}$ e $\phi^{-1}(\phi(A)) \in \mathcal{A}, \operatorname{logo} \phi(A) \in \mathcal{A}^{\prime}$. Nesse caso,

$$
\mu^{\prime}(\phi(A))=\mu\left(\phi^{-1}(\phi(A))\right)=\mu(A) .
$$

Por outro lado, seja $\phi: X \rightarrow X^{\prime}$ uma bijeção tal que, para todo $A \subset X$, $\phi(A) \in \mathcal{A}^{\prime}$ se e somente se $A \in \mathcal{A}$, e nesse caso, $\mu^{\prime}(\phi(A))=\mu(A)$. Se $A \in \mathcal{A}^{\prime}$ então $\phi\left(\phi^{-1}(A)\right) \in \mathcal{A}^{\prime}, \operatorname{logo} \phi^{-1}(A) \in \mathcal{A}$, e portanto $A \in \phi_{*} \mathcal{A}$, ou seja, $\mathcal{A}^{\prime} \subset \phi_{*} \mathcal{A}$. Além disso, para cada $A \in \mathcal{A}^{\prime}$,

$$
\mu^{\prime}(A)=\mu^{\prime}\left(\phi\left(\phi^{-1}(A)\right)\right)=\mu\left(\phi^{-1}(A)\right) .
$$

Isso mostra que $\phi$ é uma aplicação que preserva medida. Ainda, se $A \in \phi_{*} \mathcal{A}$ então $\phi^{-1}(A) \in \mathcal{A}, \operatorname{logo} \phi\left(\phi^{-1}(A)\right)=A \in \mathcal{A}^{\prime}$. Portanto $\phi_{*} \mathcal{A} \subset \mathcal{A}^{\prime}$, o que implica $\mathcal{A}^{\prime}=\phi_{*} \mathcal{A}$. Assim, $\phi$ é uma aplicação quociente, e conseqüentemente um isomorfismo.

Dito de outra maneira, um isomorfismo entre espaços de medida é uma bijeção mensurável que preserva medida e cuja inversa também é mensurável.

ObservaÇão 1.4.49. Sejam $(X, \mathcal{A}, \mu)$ e $\left(X^{\prime}, \mathcal{A}^{\prime}, \mu^{\prime}\right)$ espaços de medida e $\left(X^{\prime \prime}, \mathcal{A}^{\prime \prime}\right)$ um espaço mensurável. Se $\phi: X \rightarrow X^{\prime}$ é uma aplicação quociente, 
então uma função $f: X^{\prime} \rightarrow X^{\prime \prime}$ é mensurável se e somente se $f \circ \phi$ é mensurável. Com efeito, se $\phi$ é uma aplicação quociente então $\phi$ é mensurável. Assim, sendo $f$ é mensurável, pela Observação 1.4.33, $f \circ \phi$ também é mensurável. Reciprocamente, se $f \circ \phi$ é mensurável então, para todo $A \in \mathcal{A}^{\prime \prime}$, temos $\phi^{-1}\left(f^{-1}(A)\right)=(f \circ \phi)^{-1}(A) \in \mathcal{A}$. Portanto $f^{-1}(A) \in \phi_{*} \mathcal{A}=\mathcal{A}^{\prime}, \mathrm{o}$ que mostra que $f$ é mensurável. O diagrama seguinte ajuda a visualizar o que ocorre:

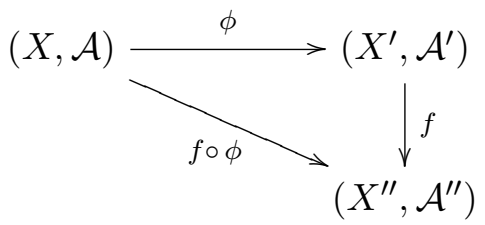

ObSERVAÇÃo 1.4.50. Se $(X, \mathcal{A}, \mu)$ é um espaço de medida e $\sim$ é uma relação de equivalência em $X$, temos uma aplicação canônica

$$
\mathfrak{q}: X \ni x \longmapsto[x] \in X / \sim
$$

e podemos munir o conjunto quociente $X / \sim \operatorname{com}$ a $\sigma$-álgebra $\mathfrak{q}_{*} \mathcal{A}$ e a medida $\mathfrak{q}_{*} \mu$, co-induzidas por $\mathfrak{q}$. Assim, a aplicação

$$
\mathfrak{q}:(X, \mathcal{A}, \mu) \longrightarrow\left(X / \sim, \mathfrak{q}_{*} \mathcal{A}, \mathfrak{q}_{*} \mu\right)
$$

torna-se claramente uma aplicação quociente.

Se $X$ é um conjunto arbitrário e $A$ um subconjunto de $X$, lembramos que a função característica de $A$ é a aplicação $\chi_{A}: X \longrightarrow \mathbb{R}$ definida por

$$
\chi_{A}(x)= \begin{cases}1 & \text { se } x \in A \\ 0 & \text { se } x \notin A .\end{cases}
$$

Dados um espaço mensurável $(X, \mathcal{A})$ e $A \subset X$, temos que $\chi_{A}$ é mensurável se e somente se $A$ é mensurável.

Lembramos ainda que uma função é chamada de simples se o seu conjunto imagem é finito. Se $\varphi: X \rightarrow \overline{\mathbb{R}}$ é uma função simples e o conjunto imagem de $\varphi$ é $\varphi(X)=\left\{a_{1}, \ldots, a_{n}\right\}$, podemos escrever

$$
\varphi=\sum_{i=1}^{n} a_{i} \chi_{A_{i}}
$$


em que $A_{i}=\varphi^{-1}\left(a_{i}\right)$ para cada $i \in\{1, \ldots, n\}$. De modo equivalente:

$$
\varphi=\sum_{c \in \varphi(X)} c \chi_{\varphi^{-1}(c)}
$$

OBSERVAÇÃo 1.4.51. Uma seqüência de funções $\left(f_{n}: X \rightarrow \overline{\mathbb{R}}\right)_{n \geq 1}$ é dita crescente (resp., decrescente) quando, para todo $x \in X, f_{n}(x) \leq f_{n+1}(x)$ (resp., $f_{n}(x) \geq f_{n+1}(x)$ ). Dada uma função $f: X \rightarrow \overline{\mathbb{R}}$, escrevemos $f_{n} \nearrow f$ (resp., $f_{n} \searrow f$ ) para expressar que $\left(f_{n}\right)_{n \geq 1}$ é uma seqüência crescente (resp., decrescente) de funções cujo limite é a função $f$, i.e., $\lim _{n \rightarrow+\infty} f_{n}(x)=f(x)$, para todo $x \in X$.

Teorema 1.4.52. Se $(X, \mathcal{A}, \mu)$ é um espaço de medida e $f: X \rightarrow \overline{\mathbb{R}}$ é uma função mensurável e não negativa, então existe uma sequência crescente $\left(\varphi_{n}: X \rightarrow \overline{\mathbb{R}}\right)_{n \geq 1}$ de funções simples, mensuráveis e não negativas tais que $\varphi_{n} \longrightarrow f$, i.e., $f(x)=\lim _{n \rightarrow+\infty} \varphi_{n}(x)$, para cada $x \in X$.

DemonstraÇão. Vide [8].

DeFiniçÃo 1.4.53. Sejam $(X, \mathcal{A}, \mu)$ um espaço de medida e $\varphi: X \rightarrow \overline{\mathbb{R}}$ uma função simples, mensurável e não negativa. A integral de $\varphi$ é definida por:

$$
\int_{X} \varphi \mathrm{d} \mu=\sum_{c \in \varphi(X)} c \mu\left(\varphi^{-1}(c)\right) .
$$

DefiniÇÃo 1.4.54. Sejam $(X, \mathcal{A}, \mu)$ um espaço de medida e $f: X \rightarrow \overline{\mathbb{R}}$ uma função mensurável e não negativa. A integral de $f$ é definida por

$$
\int_{X} f \mathrm{~d} \mu=\sup \mathcal{I}(f)
$$

$\mathcal{I}(f)=\left\{\int_{X} \varphi \mathrm{d} \mu \mid \varphi: X \rightarrow \overline{\mathbb{R}}\right.$ função simples, mensurável e $\left.0 \leq \varphi \leq f\right\}$.

DeFinição 1.4.55. Sejam $(X, \mathcal{A}, \mu)$ um espaço de medida, $Y \in \mathcal{A}$ e $f: X \rightarrow \overline{\mathbb{R}}$ uma função mensurável e não negativa. Seja ainda $\left(Y, \mathcal{A}^{\prime}, \mu^{\prime}\right)$ o espaço de medida em que $\mathcal{A}^{\prime}=\left.\mathcal{A}\right|_{Y}$ e $\mu^{\prime}=\left.\mu\right|_{\mathcal{A}^{\prime}}$. A integral de $f$ em $Y$ é definida por:

$$
\int_{Y} f \mathrm{~d} \mu=\left.\int_{Y} f\right|_{Y} \mathrm{~d} \mu^{\prime}
$$


DeFinição 1.4.56. Seja $(X, \mathcal{A}, \mu)$ um espaço de medida. Uma função $f: X \rightarrow \overline{\mathbb{R}}$ é chamada de quase integrável se é mensurável e se a diferença

$$
\int_{X} f^{+} \mathrm{d} \mu-\int_{X} f^{-} \mathrm{d} \mu
$$

está bem definida, ou seja, se $\int_{X} f^{+} \mathrm{d} \mu<+\infty$ ou $\int_{X} f^{-} \mathrm{d} \mu<+\infty$. Se $f$ é quase integrável então a integral de $f$ é definida por:

$$
\int_{X} f \mathrm{~d} \mu=\int_{X} f^{+} \mathrm{d} \mu-\int_{X} f^{-} \mathrm{d} \mu \in \overline{\mathbb{R}} .
$$

Se $f$ é quase integrável e $\int_{X} f \mathrm{~d} \mu \in \mathbb{R}$, i.e., $\int_{X} f^{+} \mathrm{d} \mu<+\infty$ e $\int_{X} f^{-} \mathrm{d} \mu<+\infty$, dizemos que $f$ é uma função integrável.

TEOrema 1.4.57 (da convergência monótona). Seja $(X, \mathcal{A}, \mu)$ um espaço de medida. Se $\left(f_{n}: X \rightarrow \overline{\mathbb{R}}\right)_{n \geq 1}$ é uma seqüência crescente de funções mensuráveis e não negativas, cujo limite é uma função $f: X \rightarrow \overline{\mathbb{R}}$, então

$$
\int_{X} f_{n} \mathrm{~d} \mu \longrightarrow \int_{X} f \mathrm{~d} \mu, \text { i.e., } \int_{X} f \mathrm{~d} \mu=\lim _{n \rightarrow+\infty} \int_{X} f_{n} \mathrm{~d} \mu \text {. }
$$

Proposição 1.4.58. Seja $(X, \mathcal{A})$ um espaço mensurável. Sejam ainda $\mu, \nu: \mathcal{A} \rightarrow[0,+\infty]$ duas medidas em $\mathcal{A} \operatorname{com} \mu(A) \leq \nu(A)$ para todo $A \in \mathcal{A}$. Se $f: X \rightarrow \mathbb{R}$ é uma função mensurável e não negativa então

$$
\int_{X} f \mathrm{~d} \mu \leq \int_{X} f \mathrm{~d} \nu
$$

DemonstraÇÃo. Se $\varphi: X \rightarrow \mathbb{R}$ uma função simples, mensurável e não negativa então:

$$
\int_{X} \varphi \mathrm{d} \mu=\sum_{c \in \varphi(X)} c \mu\left(\varphi^{-1}(c)\right) \leq \sum_{c \in \varphi(X)} c \nu\left(\varphi^{-1}(c)\right)=\int_{X} \varphi \mathrm{d} \nu .
$$

Se $f: X \rightarrow \mathbb{R}$ é uma função mensurável e não negativa então existe uma seqüência crescente $\left(\varphi_{n}: X \rightarrow \mathbb{R}\right)_{n \geq 1}$, de funções simples, mensuráveis e não negativas, tal que $\varphi_{n} \longrightarrow f$. Assim, pelo teorema da convergência monótona,

$$
\int_{X} \varphi_{n} \mathrm{~d} \mu \longrightarrow \int_{X} f \mathrm{~d} \mu \quad \text { e } \int_{X} \varphi_{n} \mathrm{~d} \nu \longrightarrow \int_{X} f \mathrm{~d} \nu .
$$

Portanto, uma vez que $\int_{X} \varphi_{n} \mathrm{~d} \mu \leq \int_{X} \varphi_{n} \mathrm{~d} \nu$ para todo $n \geq 1$, segue que

$$
\int_{X} f \mathrm{~d} \mu \leq \int_{X} f \mathrm{~d} \nu
$$

ProposiçÃo 1.4.59. Sejam $(X, \mathcal{A}, \mu)$ um espaço de medida e $f: X \rightarrow \overline{\mathbb{R}}$ uma função mensurável e não negativa. 
(a) $\int_{X} f \mathrm{~d} \mu=0$ se e somente se $f=0 \mu$-qs;

(b) $S e E, F \in \mathcal{A}$ e $E \subset F$ então $\int_{E} f \mathrm{~d} \mu \leq \int_{F} f \mathrm{~d} \mu$.

Demonstração. Vide [3].

ProposiçÃo 1.4.60. Sejam $(X, \mathcal{A}, \mu)$ um espaço de medida e $f: X \rightarrow \overline{\mathbb{R}}$ uma função mensurável.

(a) $f$ é integrável se e somente se $|f|$ é integrável;

(b) Se $f$ é quase integrável então $\left|\int_{X} f \mathrm{~d} \mu\right| \leq \int_{X}|f| \mathrm{d} \mu$.

ProposiçÃo 1.4.61. Sejam $(X, \mathcal{A}, \mu)$ um espaço de medida e $f, g: X \rightarrow \overline{\mathbb{R}}$ funções quase integráveis.

(a) Para todo $c \in \mathbb{R}$, cf é quase integrável e $\int_{X} c f \mathrm{~d} \mu=c \int_{X} f \mathrm{~d} \mu$;

(b) Se as somas $\int_{X} f \mathrm{~d} \mu+\int_{X} g \mathrm{~d} \mu$ e $f+g$ estiverem bem definidas, então $f+g$ é quase integrável $e$

$$
\int_{X}(f+g) \mathrm{d} \mu=\int_{X} f \mathrm{~d} \mu+\int_{X} g \mathrm{~d} \mu
$$

(c) Se $f \leq g$ então $\int_{X} f \mathrm{~d} \mu \leq \int_{X} g \mathrm{~d} \mu$.

Proposição 1.4.62. Sejam $(X, \mathcal{A}, \mu)$ um espaço de medida, $f: X \rightarrow \overline{\mathbb{R}}$ uma função e $Y$ um subconjunto mensurável de $X$.

(a) $\left.f\right|_{Y}$ é mensurável se e somente se $f \chi_{Y}$ é mensurável;

(b) $\left.f\right|_{Y}$ é quase integrável se e somente se $f \chi_{Y}$ é quase integrável, e nesse caso temos $\int_{Y} f \mathrm{~d} \mu=\int_{X} f \chi_{Y} \mathrm{~d} \mu$;

(c) Se $f$ é quase integrável então $\left.f\right|_{Y}$ é quase integrável;

(d) Se $X_{1}, \ldots, X_{n}$ são elementos de $\mathcal{A}$, dois a dois disjuntos, tais que $X=\bigcup_{i=1}^{n} X_{i},\left.f\right|_{X_{i}}$ é quase integrável para todo $i \in\{1, \ldots, n\}$, e se $\int_{X_{1}} f \mathrm{~d} \mu+\cdots+\int_{X_{n}} f \mathrm{~d} \mu \in \overline{\mathbb{R}}$, então $f$ é quase integrável $e$

$$
\int_{X} f \mathrm{~d} \mu=\int_{X_{1}} f \mathrm{~d} \mu+\cdots+\int_{X_{n}} f \mathrm{~d} \mu
$$

(e) Se $f$ é quase integrável, $A$ e $B$ são subconjuntos mensuráveis disjuntos de $X$ e a soma $\int_{A} f \mathrm{~d} \mu+\int_{B} f \mathrm{~d} \mu$ está em $\overline{\mathbb{R}}$, então

$$
\int_{A \cup B} f \mathrm{~d} \mu=\int_{A} f \mathrm{~d} \mu+\int_{B} f \mathrm{~d} \mu .
$$

Proposição 1.4.63. Sejam $(X, \mathcal{A}, \mu)$ um espaço de medida e $f, g: X \rightarrow \overline{\mathbb{R}}$ duas funções mensuráveis. 
(a) Se $\mu(X)=0$ então $\int_{X} f \mathrm{~d} \mu=0$;

(b) $S$ e $E \in \mathcal{A}$ e $\mu(E)=0$ então $\int_{E} f \mathrm{~d} \mu=0$;

(c) $S e A \in \mathcal{A}$ e $\mu\left(A^{c}\right)=0$ então, $f$ é quase integrável se e somente se $\left.f\right|_{A}$ é quase integrável, e nesse caso $\int_{X} f \mathrm{~d} \mu=\int_{A} f \mathrm{~d} \mu$;

(d) Se $f=g \mu$-qs então, $f$ é quase integrável se e somente se $g$ é quase integrável, e nesse caso $\int_{X} f \mathrm{~d} \mu=\int_{X} g \mathrm{~d} \mu$.

Lema 1.4.64. Sejam $(X, \mathcal{A}, \mu)$ e $\left(X^{\prime}, \mathcal{A}^{\prime}, \mu^{\prime}\right)$ dois espaços de medida e $\phi: X \rightarrow X^{\prime}$ uma aplicação que preserva medida. Se $\varphi: X^{\prime} \rightarrow \overline{\mathbb{R}}$ é uma função simples, mensurável e não negativa então

$$
\int_{X^{\prime}} \varphi \mathrm{d} \mu^{\prime}=\int_{X}(\varphi \circ \phi) \mathrm{d} \mu .
$$

Demonstração. Se $\varphi: X^{\prime} \rightarrow \overline{\mathbb{R}}$ é uma função simples, mensurável e não negativa então $\varphi \circ \phi$ também é simples, mensurável e não negativa. Além disso,

$$
\begin{aligned}
& \int_{X^{\prime}} \varphi \mathrm{d} \mu^{\prime}=\sum_{c \in \varphi\left(X^{\prime}\right)} c \mu^{\prime}\left(\varphi^{-1}(c)\right)=\sum_{c \in \varphi\left(X^{\prime}\right)} c \mu\left(\phi^{-1}\left(\varphi^{-1}(c)\right)\right) \\
& =\sum_{c \in \varphi\left(X^{\prime}\right)} c \mu\left((\varphi \circ \phi)^{-1}(c)\right)=\sum_{c \in(\varphi \circ \phi)(X)} c \mu\left((\varphi \circ \phi)^{-1}(c)\right)=\int_{X}(\varphi \circ \phi) \mathrm{d} \mu,
\end{aligned}
$$

sendo a penúltima igualdade verdadeira porque $(\varphi \circ \phi)^{-1}(c)=\emptyset$ para cada $c \in \varphi\left(X^{\prime}\right) \backslash(\varphi \circ \phi)(X)$.

Lema 1.4.65. Sejam $(X, \mathcal{A}, \mu)$ e $\left(X^{\prime}, \mathcal{A}^{\prime}, \mu^{\prime}\right)$ dois espaços de medida $e$ $\phi: X \rightarrow X^{\prime}$ uma aplicação que preserva medida. Se $f: X^{\prime} \rightarrow \overline{\mathbb{R}}$ é uma função mensurável e não negativa então

$$
\int_{X^{\prime}} f \mathrm{~d} \mu^{\prime}=\int_{X}(f \circ \phi) \mathrm{d} \mu
$$

DemonstraÇÃo. Se $f: X^{\prime} \rightarrow \overline{\mathbb{R}}$ é uma função mensurável e não negativa então, pelo Teorema 1.4.52, existe uma sequência crescente $\left(\varphi_{n}\right)_{n \geq 1}$, $\varphi_{n}: X^{\prime} \rightarrow \overline{\mathbb{R}}$, de funções simples, mensuráveis e não negativas, tal que $\varphi_{n} \longrightarrow f$. Disso decorre que $\left(\varphi_{n} \circ \phi: X \rightarrow \overline{\mathbb{R}}\right)_{n \geq 1}$ é uma seqüência de funções simples, mensuráveis e não negativas tal que $\varphi_{n} \circ \phi \longrightarrow f \circ \phi$. Assim, pelo teorema da convergência monótona, temos:

$$
\int_{X^{\prime}} \varphi_{n} \mathrm{~d} \mu^{\prime} \longrightarrow \int_{X^{\prime}} f \mathrm{~d} \mu^{\prime} \text { e } \int_{X}\left(\varphi_{n} \circ \phi\right) \mathrm{d} \mu \longrightarrow \int_{X}(f \circ \phi) \mathrm{d} \mu .
$$


Mas, pelo Lema 1.4.64, $\int_{X^{\prime}} \varphi_{n} \mathrm{~d} \mu^{\prime}=\int_{X}\left(\varphi_{n} \circ \phi\right) \mathrm{d} \mu$ para todo $n \geq 1$, logo

$$
\int_{X^{\prime}} f \mathrm{~d} \mu^{\prime}=\int_{X}(f \circ \phi) \mathrm{d} \mu \text {. }
$$

Proposição 1.4.66. Sejam $(X, \mathcal{A}, \mu)$ e $\left(X^{\prime}, \mathcal{A}^{\prime}, \mu^{\prime}\right)$ dois espaços de medida e $\phi: X \rightarrow X^{\prime}$ uma aplicação que preserva medida. Dada uma função mensurável $f: X^{\prime} \rightarrow \overline{\mathbb{R}}$ temos que $f$ é quase integrável se e somente se $f \circ \phi$ é quase integrável, e nesse caso:

$$
\int_{X^{\prime}} f \mathrm{~d} \mu^{\prime}=\int_{X}(f \circ \phi) \mathrm{d} \mu
$$

Demonstração. Se $f: X^{\prime} \rightarrow \overline{\mathbb{R}}$ é uma função mensurável então as funções $f^{+}, f^{-}: X^{\prime} \rightarrow \overline{\mathbb{R}}$ também são mensuráveis. Assim, pelo Lema 1.4.65,

$$
\int_{X^{\prime}} f^{+} \mathrm{d} \mu^{\prime}=\int_{X}\left(f^{+} \circ \phi\right) \mathrm{d} \mu \quad \text { e } \quad \int_{X^{\prime}} f^{-} \mathrm{d} \mu^{\prime}=\int_{X}\left(f^{-} \circ \phi\right) \mathrm{d} \mu,
$$

e portanto

$$
\int_{X^{\prime}} f^{+} \mathrm{d} \mu^{\prime}=\int_{X}(f \circ \phi)^{+} \mathrm{d} \mu \quad \text { e } \quad \int_{X^{\prime}} f^{-} \mathrm{d} \mu^{\prime}=\int_{X}(f \circ \phi)^{-} \mathrm{d} \mu .
$$

Isso mostra que $f$ é quase integrável se e somente se $f \circ \phi$ é quase integrável, e além disso:

$$
\begin{aligned}
\int_{X^{\prime}} f \mathrm{~d} \mu^{\prime} & =\int_{X^{\prime}} f^{+} \mathrm{d} \mu^{\prime}-\int_{X^{\prime}} f^{-} \mathrm{d} \mu^{\prime} \\
& =\int_{X}(f \circ \phi)^{+} \mathrm{d} \mu-\int_{X}(f \circ \phi)^{-} \mathrm{d} \mu=\int_{X}(f \circ \phi) \mathrm{d} \mu .
\end{aligned}
$$

Corolário 1.4.67. Sejam $(X, \mathcal{A}, \mu)$ e $\left(X, \mathcal{A}^{\prime}, \mu^{\prime}\right)$ espaços de medida com $\mathcal{A} \subset \mathcal{A}^{\prime}$ e $\mu=\left.\mu^{\prime}\right|_{\mathcal{A}}$. Se $f: X \rightarrow \overline{\mathbb{R}}$ é uma função quase integrável então

$$
\int_{X} f \mathrm{~d} \mu^{\prime}=\int_{X} f \mathrm{~d} \mu
$$

DemonstraÇÃo. O seguinte diagrama ajuda a visualizar a demonstração:

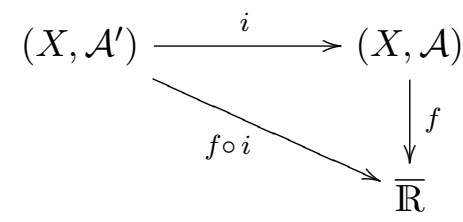

A aplicação inclusão $i:\left(X, \mathcal{A}^{\prime}\right) \rightarrow(X, \mathcal{A})$ é mensurável, e para cada $A \in \mathcal{A}$, temos que $\left(i_{*} \mu^{\prime}\right)(A)=\mu^{\prime}\left(i^{-1}(A)\right)=\mu^{\prime}(A)=\mu(A)$, ou seja, $i_{*} \mu^{\prime}=\mu$. 
Assim, pelo item (b) da Proposição 1.4.46, a aplicação $i$ preserva medida, e portanto, pela Proposição 1.4.66, segue que

$$
\int_{X} f \mathrm{~d} \mu=\int_{X}(f \circ i) \mathrm{d} \mu^{\prime}=\int_{X} f \mathrm{~d} \mu^{\prime}
$$

Lema 1.4.68. Sejam $(X, \mathcal{A}, \mu)$ um espaço de medida e $(X, \overline{\mathcal{A}}, \bar{\mu})$ o seu completamento. Para cada função $\varphi: X \rightarrow \mathbb{R}$ simples, não negativa e mensurável em relação a $\overline{\mathcal{A}}$, existe $\widetilde{\varphi}: X \rightarrow \mathbb{R}$ mensurável relativamente a $\mathcal{A} \operatorname{com} \widetilde{\varphi}=\varphi \bar{\mu}-q s$ e $\widetilde{\varphi} \leq \varphi$.

DemonstraçÃo. Dado $A \in \overline{\mathcal{A}}$, vamos mostrar inicialmente que o resultado é válido para $\chi_{A}: X \rightarrow \mathbb{R}$, a função característica de $A$. Como $A \in \overline{\mathcal{A}}$ então existem $E \in \mathcal{A}, N \in \mathcal{A} \operatorname{com} \mu(N)=0$ e $F \subset N$ tais que $A=E \cup F$. Como $E \in \mathcal{A}$, a função $\chi_{E}$ é mensurável relativamente a $\mathcal{A}$ e como $E \subset A$, vale $\chi_{E} \leq \chi_{A}$. Observe que o conjunto $\left\{x \in X: \chi_{A}(x) \neq \chi_{E}(x)\right\}$ é igual a $F \backslash E, F \backslash E$ está em $\overline{\mathcal{A}}$ (pois $E, F \in \overline{\mathcal{A}}$ ) e $\bar{\mu}(F \backslash E) \leq \bar{\mu}(N)=\mu(N)=0$; $\operatorname{logo} \chi_{E}=\chi_{A} \bar{\mu}$-qs. Seja agora $\varphi: X \rightarrow \mathbb{R}$ uma função simples, não negativa e mensurável em relação a $\overline{\mathcal{A}}$. Então, para cada $c \in \varphi(X)$, existe $E(c) \in \mathcal{A}$ tal que $\chi_{\varphi^{-1}(c)}=\chi_{E(c)} \bar{\mu}$-qs e $\chi_{\varphi^{-1}(c)} \leq \chi_{E(c)}$. Assim, a aplicação

$$
\widetilde{\varphi}=\sum_{c \in \varphi(X)} c \chi_{E(c)}
$$

é $\mathcal{A}$-mensurável, $\widetilde{\varphi} \leq \varphi$ e, pelo Corolário 1.4.43, $\widetilde{\varphi}=\varphi \bar{\mu}$-qs.

Proposição 1.4.69. Sejam $(X, \mathcal{A}, \mu)$ um espaço de medida e $(X, \overline{\mathcal{A}}, \bar{\mu})$ o seu completamento. Para cada função $f: X \rightarrow \mathbb{R}$ mensurável em relação $a \overline{\mathcal{A}}$, existe $\tilde{f}: X \rightarrow \mathbb{R}$ mensurável relativamente a $\mathcal{A}$ com $\widetilde{f}=f \bar{\mu}$-qs.

Demonstração. Seja $g: X \rightarrow \mathbb{R}$ uma função $\overline{\mathcal{A}}$-mensurável e não negativa. Então existe uma seqüência crescente $\left(\varphi_{n}: X \rightarrow \mathbb{R}\right)_{n \geq 1}$ de funções simples, $\overline{\mathcal{A}}$-mensuráveis e não negativas com $\varphi_{n} \longrightarrow g$. Para cada $n \geq 1$, pelo Lema 1.4.68, existe uma função $\psi_{n}$ mensurável em relação a $\mathcal{A}$ tal que $\psi_{n}=\varphi_{n} \bar{\mu}$-qs e $\psi_{n} \leq \varphi_{n}$. Se $\widetilde{g}=\lim \sup \psi_{n}$ então $\widetilde{g}$ é mensurável em relação a $\mathcal{A}$ e, pelo Lema 1.4 .44 , temos $\widetilde{g}=g \bar{\mu}$-qs. Como $\widetilde{g} \leq g$, temos que $\widetilde{g}(X) \subset \mathbb{R}$. Seja agora $f: X \rightarrow \mathbb{R}$ uma função mensurável em relação a $\overline{\mathcal{A}}$. Então as funções $f^{+}$e $f^{-}$também são $\overline{\mathcal{A}}$-mensuráveis, e portanto existem $\widetilde{f^{+}}$e $\widetilde{f^{-}}$mensuráveis relativamente a $\mathcal{A}$ com $\widetilde{f^{+}}=f^{+} \bar{\mu}$-qs e $\widetilde{f^{-}}=f^{-}$ 
$\bar{\mu}$-qs. Logo $\widetilde{f}=\widetilde{f^{+}}-\widetilde{f^{-}}$é uma função mensurável com respeito a $\mathcal{A}$ e, pelo Lema $1.4 .42, \tilde{f}=f \bar{\mu}$-qs.

ObservaÇÃo 1.4.70. Se $(X, \mathcal{A}, \mu)$ é um espaço de medida, $f: X \rightarrow \mathbb{R}$ é uma função mensurável e não negativa, e $\left(E_{n}\right)_{n \geq 1}$ é uma seqüência de elementos de $\mathcal{A}$, dois a dois disjuntos, com $X=\bigcup_{n \geq 1} E_{n}$, então

$$
\int_{X} f \mathrm{~d} \mu=\sum_{n \geq 1} \int_{E_{n}} f \mathrm{~d} \mu .
$$

De fato, para cada $n \geq 1$ seja $f_{n}=f \chi_{\left(\bigcup_{k=1}^{n} E_{k}\right)}$. Como $E_{i} \cap E_{j}=\emptyset$ para $i \neq j$, segue que $f_{n}=f \sum_{k=1}^{n} \chi_{E_{k}}=\sum_{k=1}^{n} f \chi_{E_{k}}$, e uma vez que $\left(f_{n}\right)_{n \geq 1}$ é uma seqüência crescente de funções mensuráveis e não negativas convergindo para $f$, pelo teorema da convergência monótona, temos:

$$
\begin{aligned}
\int_{X} f \mathrm{~d} \mu=\lim _{n \rightarrow+\infty} \int_{X} f_{n} \mathrm{~d} \mu=\lim _{n \rightarrow+\infty} \int_{X}\left(\sum_{k=1}^{n} f \chi_{E_{k}}\right) \mathrm{d} \mu \\
\quad=\lim _{n \rightarrow+\infty} \sum_{k=1}^{n}\left(\int_{E_{k}} f \mathrm{~d} \mu\right)=\sum_{n \geq 1} \int_{E_{n}} f \mathrm{~d} \mu .
\end{aligned}
$$

Proposição 1.4.71. Seja $(X, \mathcal{A}, \mu)$ um espaço de medida, em que $\mathcal{A}$ é a $\sigma$-álgebra $\mathcal{P}(X)$ e $\mu$ é a medida de contagem. Se $f: X \rightarrow \mathbb{R}$ é uma função não negativa então

$$
\int_{X} f \mathrm{~d} \mu=\sum_{x \in X} f(x) .
$$

DemonstraÇÃo. Seja $F$ um subconjunto finito de $X$. Uma vez que $F=\bigcup_{x \in F}\{x\}$, temos $f \chi_{F}=f \sum_{x \in F} \chi_{\{x\}}=\sum_{x \in F} f \chi_{\{x\}}$. Então

$$
\begin{aligned}
\int_{F} f \mathrm{~d} \mu=\int_{X} f \chi_{F} \mathrm{~d} \mu & =\int_{X}\left(\sum_{x \in F} f \chi_{\{x\}}\right) \mathrm{d} \mu=\sum_{x \in F} \int_{X} f \chi_{\{x\}} \mathrm{d} \mu \\
& =\sum_{x \in F} \int_{\{x\}} f \mathrm{~d} \mu=\sum_{x \in F}(f(x) \mu(\{x\}))=\sum_{x \in F} f(x) .
\end{aligned}
$$

Assim, para cada subconjunto finito $F \subset X$ temos

$$
\sum_{x \in F} f(x)=\int_{F} f \mathrm{~d} \mu \leq \int_{X} f \mathrm{~d} \mu .
$$

Visto que $\sum_{x \in X} f(x)=\sup \left\{\sum_{x \in F} f(x): F \subset X, F\right.$ finito $\}$, segue que

$$
\sum_{x \in X} f(x) \leq \int_{X} f \mathrm{~d} \mu .
$$


Seja agora $\varphi: X \rightarrow \mathbb{R}$ uma função simples e não negativa com $\varphi \leq f$. Então

$$
\begin{aligned}
\int_{X} \varphi \mathrm{d} \mu=\sum_{c \in \operatorname{Im} \varphi} c \mu\left(\varphi^{-1}(c)\right) & =\sum_{c \in \operatorname{Im} \varphi} c\left|\varphi^{-1}(c)\right| \\
= & \sum_{c \in \operatorname{Im} \varphi}\left(\sum_{x \in \varphi^{-1}(c)} \varphi(x)\right)=\sum_{x \in X} \varphi(x) \leq \sum_{x \in X} f(x),
\end{aligned}
$$

o que implica $\int_{X} f \mathrm{~d} \mu \leq \sum_{x \in X} f(x)$, pois

$$
\int_{X} f \mathrm{~d} \mu=\sup \left\{\int_{X} \varphi \mathrm{d} \mu \mid \varphi: X \rightarrow \mathbb{R} \text { simples, mensurável e } 0 \leq \varphi \leq f\right\} .
$$

Daí segue o resultado.

Como última parte desta seção, veremos a partir de agora os espaços $L^{p}$. Se $(X, \mathcal{A}, \mu)$ é um espaço de medida, denotamos por $\mathcal{M}(X, \mathcal{A})$ o espaço vetorial real das funções mensuráveis a valores reais sobre $X$, por $\mathcal{M}_{o}(X, \mathcal{A}, \mu)$ o subespaço de $\mathcal{M}(X, \mathcal{A})$ das funções nulas $\mu$-qs, e por $\overline{\mathcal{M}}(X, \mathcal{A}, \mu)$ o quociente de $\mathcal{M}(X, \mathcal{A})$ por $\mathcal{M}_{o}(X, \mathcal{A}, \mu)$. Ou seja,

- $\mathcal{M}(X, \mathcal{A})=\{f: X \rightarrow \mathbb{R}: f$ é mensurável $\}$,

- $\mathcal{M}_{o}(X, \mathcal{A}, \mu)=\{f \in \mathcal{M}(X, \mathcal{A}): f=0 \mu$-quase sempre $\}$,

- $\overline{\mathcal{M}}(X, \mathcal{A}, \mu)=\mathcal{M}(X, \mathcal{A}) / \mathcal{M}_{o}(X, \mathcal{A}, \mu)=\left\{[f]_{\mu}: f \in \mathcal{M}(X, \mathcal{A})\right\}$,

em que $[f]_{\mu}=\{g \in \mathcal{M}(X, \mathcal{A}): g=f \mu$-qs $\}$ (não havendo risco de confusão, escreveremos apenas $[f]$ em vez de $\left.[f]_{\mu}\right)$. Dada uma função $f \in \mathcal{M}(X, \mathcal{A})$ e $p \in[1,+\infty[$, definimos

$$
\begin{gathered}
\|f\|_{p}=\left(\int_{X}|f|^{p} \mathrm{~d} \mu\right)^{\frac{1}{p}} \in[0,+\infty], \text { e } \\
\|f\|_{\infty}=\inf \{c \in[0,+\infty]:|f| \leq c \quad \mu \text {-qs }\} \in[0,+\infty] .
\end{gathered}
$$

Para cada $p \in[1,+\infty]$ denotamos por $L^{p}(X, \mathcal{A}, \mu)$ o subconjunto do espaço $\overline{\mathcal{M}}(X, \mathcal{A}, \mu)$ cujos elementos são as classes de funções $[f]$ com $\|f\|_{p}<+\infty$, isto é,

$$
L^{p}(X, \mathcal{A}, \mu)=\left\{[f] \in \overline{\mathcal{M}}(X, \mathcal{A}, \mu):\|f\|_{p}<+\infty\right\} .
$$

Note que, para $p<+\infty$, uma função $f \in \mathcal{M}(X, \mathcal{A})$ satisfaz $[f] \in L^{p}(X, \mathcal{A}, \mu)$ se e somente se $\int_{X}|f|^{p} \mathrm{~d} \mu<+\infty$, e satisfaz $[f] \in L^{\infty}(X, \mathcal{A}, \mu)$ se e somente se $f$ é $\mu$-qs limitada. Para cada $[f] \in L^{p}(X, \mathcal{A}, \mu)$ definimos $\|[f]\|_{p}=\|f\|_{p}$. Note que o valor de $\|[f]\|_{p}$ independe do representante tomado da classe $[f]$ 
pois, se $g=f \mu$-qs então $\int_{X}|g|^{p} \mathrm{~d} \mu=\int_{X}|f|^{p} \mathrm{~d} \mu$ quando $p \in[1,+\infty[$ e, para todo $c \in[0,+\infty],|g| \leq c \mu$-qs se e somente se $|f| \leq c \mu$-qs. Veremos a seguir as desigualdades de Hölder e Minkowski. A desigualdade de Hölder é usada para provar a de Minkowski, e esta última implica que, para cada $p \in[1,+\infty], L^{p}(X, \mathcal{A}, \mu)$ é subespaço vetorial de $\overline{\mathcal{M}}(X, \mathcal{A}, \mu)$, e que $\|\cdot\|_{p}$ é uma norma em $L^{p}(X, \mathcal{A}, \mu)$. Por abuso de linguagem, diremos que uma função $f$ está em $L^{p}(X, \mathcal{A}, \mu)$ significando que a classe de aplicações iguais a $f \mu$-qs está em $L^{p}(X, \mathcal{A}, \mu)$.

OBSERVAÇÃo 1.4.72. Se $p, q \in[1,+\infty]$ são tais que $\frac{1}{p}+\frac{1}{q}=1$ então $p$ e $q$ são chamados de expoentes conjugados.

Lema 1.4.73 (desigualdade de Hölder). Seja $(X, \mathcal{A}, \mu)$ um espaço de medida. Se $f \in L^{p}(X, \mathcal{A}, \mu)$ e $g \in L^{q}(X, \mathcal{A}, \mu)$, com $1 \leq p<+\infty$ e q dado por $\frac{1}{p}+\frac{1}{q}=1$, então $f g \in L^{1}(X, \mathcal{A}, \mu)$ e $\|f g\|_{1} \leq\|f\|_{p}\|g\|_{q}$.

Demonstração. Vide [3].

Lema 1.4.74 (desigualdade de Minkowski). Sejam $(X, \mathcal{A}, \mu)$ um espaço de medida e $p \in[1,+\infty]$. Se $f, g \in L^{p}(X, \mathcal{A}, \mu)$ então $f+g \in L^{p}(X, \mathcal{A}, \mu)$ e $\|f+g\|_{p} \leq\|f\|_{p}+\|g\|_{p}$.

DemonstraçÃo. Vide [8].

Corolário 1.4.75. Se $(X, \mathcal{A}, \mu)$ é um espaço de medida e $p \in[1,+\infty]$ então $L^{p}(X, \mathcal{A}, \mu)$ é um subespaço vetorial de $\overline{\mathcal{M}}(X, \mathcal{A}, \mu)$ e \|\|$_{p}$ é uma norma em $L^{p}(X, \mathcal{A}, \mu)$.

Teorema 1.4.76. Se $(X, \mathcal{A}, \mu)$ é um espaço de medida e $p \in[1,+\infty]$ então $\left(L^{p}(X, \mathcal{A}, \mu),\|\cdot\|_{p}\right)$ é um espaço de Banach.

DemonstraçÃo. Vide [3].

OBSERVAÇÃo 1.4.77. Se $f \in L^{\infty}(X, \mathcal{A}, \mu)$ então a sua norma, definida por $\|f\|_{\infty}=\inf A$, em que $A=\{c \in[0,+\infty]:|f| \leq c \mu$-qs $\}$, também é igual ao mínimo de $A$. Com efeito, se $\alpha=\|f\|_{\infty}=\inf A$ então para cada inteiro $n \geq 1$ existe $k \in \mathbb{R}, \alpha<k<\alpha+\frac{1}{n}$, tal que $|f| \leq k \mu$-qs. Assim, como

$$
E_{n} \stackrel{\text { def }}{=}\left\{x \in X:|f(x)|>\alpha+\frac{1}{n}\right\} \subset F \stackrel{\text { def }}{=}\{x \in X:|f(x)|>k\},
$$


temos que $\mu\left(E_{n}\right) \leq \mu(F)=0$, ou seja, $\mu\left(E_{n}\right)=0$ para todo $n \geq 1$. Por outro lado, o conjunto $A_{\alpha}=\{x \in X:|f(x)|>\alpha\}$ é igual a $\bigcup_{n \geq 1} E_{n}$, logo

$$
\mu\left(A_{\alpha}\right) \leq \sum_{n \geq 1} \mu\left(E_{n}\right)=0, \text { i.e., } \mu\left(A_{\alpha}\right)=0 .
$$

Segue que $|f| \leq \alpha \mu$-qs, e portanto $\alpha \in A$. Isso mostra que $\alpha=\min A$.

OBservaÇÃo 1.4.78. Se $p \in\left[1,+\infty\left[\right.\right.$ e $\mathfrak{a}=\left(a_{i}\right)_{i \in I}$ é uma família de números reais em $L^{p}(I, \mathcal{P}(I), \mu)$, em que $\mu$ é a medida de contagem, então

$$
\|\mathfrak{a}\|_{p}=\left(\sum_{i \in I}\left|a_{i}\right|^{p}\right)^{\frac{1}{p}} .
$$

De fato, pela Proposição 1.4.71, temos:

$$
\|\mathfrak{a}\|_{p}=\left(\int_{I}|a|^{p} \mathrm{~d} \mu\right)^{\frac{1}{p}}=\left(\sum_{i \in I}\left|a_{i}\right|^{p}\right)^{\frac{1}{p}} .
$$

Além disso, se $\mathfrak{b}=\left(b_{i}\right)_{i \in I}$ é uma família de números reais em $L^{p}(I, \mathcal{P}(I), \mu)$, com $\left|a_{i}\right| \leq\left|b_{i}\right|$ para todo $i \in I$, então

$$
\|\mathfrak{a}\|_{p}=\left(\sum_{i \in I}\left|a_{i}\right|^{p}\right)^{\frac{1}{p}} \leq\left(\sum_{i \in I}\left|b_{i}\right|^{p}\right)^{\frac{1}{p}}=\|\mathfrak{b}\|_{p},
$$

ou seja, $\|\mathfrak{a}\|_{p} \leq\|\mathfrak{b}\|_{p}$.

Se $(X, \mathcal{A}, \mu)$ é um espaço de medida arbitrário e $Y$ é um subconjunto mensurável de $X$ então, conforme vimos anteriormente, a terna $\left(Y, \mathcal{A}^{\prime}, \mu^{\prime}\right)$, na qual $\mathcal{A}^{\prime}=\left.\mathcal{A}\right|_{Y}(=\mathcal{A} \cap \mathcal{P}(Y))$ e $\mu^{\prime}=\left.\mu\right|_{\mathcal{A}^{\prime}}$, é um espaço de medida. Em outras palavras, todo subconjunto mensurável de $X$, munido com a $\sigma$-álgebra dos conjuntos mensuráveis de $X$ nele contidos e com a restrição da medida a essa $\sigma$-álgebra, é, ele próprio, um espaço de medida. Veremos que para todo $p \in[1,+\infty]$, o espaço $L^{p}\left(Y, \mathcal{A}^{\prime}, \mu^{\prime}\right)$ pode ser (isometricamente) identificado com o subespaço de $L^{p}(X, \mathcal{A}, \mu)$ cujos elementos são classes de aplicações que admitem um representante que se anula fora de $Y$.

Lema 1.4.79. Sejam $(X, \mathcal{A}, \mu)$ um espaço de medida e $Y$ um subconjunto mensurável de $X$. Sejam ainda $\mathcal{A}^{\prime}=\left.\mathcal{A}\right|_{Y}, \mu^{\prime}=\left.\mu\right|_{\mathcal{A}^{\prime}}$ e $p \in[1,+\infty]$. Se $f \in L^{p}\left(Y, \mathcal{A}^{\prime}, \mu^{\prime}\right)$ e $\bar{f}: X \rightarrow \mathbb{R}$ é uma função definida por $\bar{f}(x)=f(x)$ se $x \in Y$ e $\bar{f}(x)=0$ se $x \notin Y$, então $\bar{f} \in L^{p}(X, \mathcal{A}, \mu)$. 
Demonstração. Pela Proposição 1.4.35, $\bar{f}$ é mensurável com respeito a $\mathcal{A}$, i.e., $f \in \mathcal{M}(X, \mathcal{A})$. Se $p<+\infty$ então

$$
\int_{X}|\bar{f}|^{p} \mathrm{~d} \mu=\int_{Y}|\bar{f}|^{p} \mathrm{~d} \mu+\int_{Y^{c}}|\bar{f}|^{p} \mathrm{~d} \mu=\int_{Y}|f|^{p} \mathrm{~d} \mu^{\prime}+0=\int_{Y}|f|^{p} \mathrm{~d} \mu^{\prime}<+\infty .
$$

$\operatorname{Logo} \bar{f} \in L^{p}(X, \mathcal{A}, \mu)$ e $\left\|[\bar{f}]_{\mu}\right\|_{p}=\left\|[f]_{\mu^{\prime}}\right\|_{p}$. Seja $c \in[0,+\infty]$. Afirmamos que $|f| \leq c \mu^{\prime}$-qs se e somente se $|\bar{f}| \leq c \mu$-qs. De fato,

$$
\begin{aligned}
\mu(\{x \in X:|\bar{f}(x)|>c\}) & =\mu(\{x \in Y:|\bar{f}(x)|>c\})+\mu\left(\left\{x \in Y^{c}:|\bar{f}(x)|>c\right\}\right) \\
& =\mu^{\prime}(\{x \in Y:|f(x)|>c\})+\mu(\emptyset) \\
& =\mu^{\prime}(\{x \in Y:|f(x)|>c\}) .
\end{aligned}
$$

Portanto, se $A \stackrel{\text { def }}{=}\{x \in X:|\bar{f}(x)|>c\}$ e $B \stackrel{\text { def }}{=}\{x \in Y:|f(x)|>c\}$ então $\mu(A)=0$ se e somente se $\mu^{\prime}(B)=0$, ou seja, $|\bar{f}| \leq c \mu$-qs se e somente se $|f| \leq c \mu^{\prime}$-qs. Logo $\left\|[\bar{f}]_{\mu}\right\|_{\infty}=\left\|[f]_{\mu^{\prime}}\right\|_{\infty}<+\infty$, o que mostra que a aplicação $\bar{f}$ está em $L^{\infty}(X, \mathcal{A}, \mu)$.

Proposição 1.4.80. Sejam $(X, \mathcal{A}, \mu)$ um espaço de medida, $Y$ um subconjunto mensurável de $X, \mathcal{A}^{\prime}=\left.\mathcal{A}\right|_{Y}$ e $\mu^{\prime}=\left.\mu\right|_{\mathcal{A}^{\prime}}$. Para cada $p \in[1,+\infty]$, a aplicação

$$
L^{p}\left(Y, \mathcal{A}^{\prime}, \mu^{\prime}\right) \ni[f]_{\mu^{\prime}} \longmapsto[\bar{f}]_{\mu} \in L^{p}(X, \mathcal{A}, \mu)
$$

é uma imersão isométrica ( $\bar{f}$ como no enunciado do Lema 1.4.79).

DemonstraÇão. Seja $\phi$ a aplicação (1.4.1).

- $\phi$ está bem definida.

Para cada $f \in L^{p}\left(Y, \mathcal{A}^{\prime}, \mu^{\prime}\right)$ temos $\bar{f} \in L^{p}(X, \mathcal{A}, \mu)$, pelo Lema 1.4.79. Sejam $f, g \in L^{p}\left(Y, \mathcal{A}^{\prime}, \mu^{\prime}\right)$ tais que $[f]_{\mu^{\prime}}=[g]_{\mu^{\prime}}$. Então $f=g \mu^{\prime}$-qs, ou seja, existe $B \in \mathcal{A}^{\prime}$ com $\mu^{\prime}(B)=0$ tal que $f(x)=g(x)$ para todo $x \in Y \backslash B$. Como $B \subset Y$ temos $\bar{f}(x)=\bar{g}(x)$ para todo $x \in X \backslash B$. Mas $B \in \mathcal{A}$ e $\mu(B)=0, \operatorname{logo} \bar{f}=\bar{g} \mu$-qs, i.e., $[\bar{f}]_{\mu}=[\bar{g}]_{\mu}$.

- $\phi$ é linear.

Sejam $\alpha \in \mathbb{R}$ e $f, g \in L^{p}\left(Y, \mathcal{A}^{\prime}, \mu^{\prime}\right)$. Então:

$$
\begin{aligned}
\phi\left(\alpha[f]_{\mu^{\prime}}+[g]_{\mu^{\prime}}\right) & =\phi\left([\alpha f+g]_{\mu^{\prime}}\right)=[\overline{\alpha f+g}]_{\mu} \\
& =[\alpha \bar{f}+\bar{g}]_{\mu}=\alpha[\bar{f}]_{\mu}+[\bar{g}]_{\mu}=\alpha \phi\left([f]_{\mu^{\prime}}\right)+\phi\left([g]_{\mu^{\prime}}\right) .
\end{aligned}
$$


- $\phi$ preserva norma.

Se $f \in L^{p}\left(Y, \mathcal{A}^{\prime}, \mu^{\prime}\right)$ então, pelo Lema 1.4.79, $\left\|[\bar{f}]_{\mu}\right\|_{p}=\left\|[f]_{\mu^{\prime}}\right\|_{p}$ para todo $p \in[1,+\infty]$.

Portanto $\phi$ é uma imersão isométrica.

Proposição 1.4.81. A imagem da aplicação (1.4.1) é o subespaço de $L^{p}(X, \mathcal{A}, \mu)$ das classes de aplicações que admitem um representante que se anula em $X \backslash Y$.

Demonstração. Chamemos de $\phi$ a aplicação (1.4.1). Vamos mostrar que a imagem de $\phi$ é o conjunto

$$
\mathcal{B}=\left\{[f]_{\mu} \in L^{p}(X, \mathcal{A}, \mu) \text { : existe } g \in[f]_{\mu} \operatorname{com} g_{\left.\right|_{Y^{c}}}=0\right\} .
$$

Se $f \in L^{p}\left(Y, \mathcal{A}^{\prime}, \mu^{\prime}\right)$ então $\bar{f} \in L^{p}(X, \mathcal{A}, \mu)$ e $\left.\bar{f}\right|_{Y^{c}}=0 ; \operatorname{logo} \phi\left([f]_{\mu^{\prime}}\right)=[\bar{f}]_{\mu}$ está em $\mathcal{B}$, i.e., $\operatorname{Im}(\phi) \subset \mathcal{B}$. Por outro lado, se $[f]_{\mu} \in \mathcal{B}$ então existe $g \in[f]_{\mu}$ com $g_{\left.\right|_{Y c}}=0$. Afirmamos que $g_{\left.\right|_{Y}} \in L^{p}\left(Y, \mathcal{A}^{\prime}, \mu^{\prime}\right)$ e $\phi\left(\left[g_{\left.\right|_{Y}}\right]_{\mu^{\prime}}\right)=[f]_{\mu}$. Com efeito, se $E$ é boreleano arbitrário de $\mathbb{R}$ então $\left(g_{\left.\right|_{Y}}\right)^{-1}(E)=g^{-1}(E) \cap Y \in \mathcal{A}^{\prime}$ (pois $g^{-1}(E) \in \mathcal{A}$ ), o que implica na $\mathcal{A}^{\prime}$-mensurabilidade de $g_{\left.\right|_{Y}}$. Além disso, como $g_{\left.\right|_{Y^{c}}}=0$, para cada $p<+\infty$ temos:

$$
\left\|\left[g_{\left.\right|_{Y}}\right]_{\mu^{\prime}}\right\|_{p}^{p}=\int_{Y}\left|g_{\left.\right|_{Y}}\right|^{p} \mathrm{~d} \mu^{\prime}=\int_{Y}|g|^{p} \mathrm{~d} \mu+\int_{Y^{c}}|g|^{p} \mathrm{~d} \mu=\int_{X}|g|^{p} \mathrm{~d} \mu<+\infty .
$$

Portanto $g_{\left.\right|_{Y}} \in L^{p}\left(Y, \mathcal{A}^{\prime}, \mu^{\prime}\right)$ para todo $p \in[1,+\infty[$. No caso de $p=+\infty$, para verificar que $g_{\left.\right|_{Y}}$ está em $L^{\infty}\left(Y, \mathcal{A}^{\prime}, \mu^{\prime}\right)$, observamos que do corpo da demonstração do Lema 1.4.79, temos $\left|g_{\left.\right|_{Y}}\right| \leq c \mu^{\prime}$-qs se e somente se $\left|\overline{g_{\mid Y}}\right| \leq c$ $\mu$-qs, para todo $c \in[0,+\infty]$. Assim, como $\overline{g_{\mid Y}}(x)=g(x)$ para todo $x \in X$, segue que $\left\|\left[g_{\left.\right|_{Y}}\right]_{\mu^{\prime}}\right\|_{\infty}=\left\|[g]_{\mu}\right\|_{\infty}<+\infty$, e portanto $g_{\left.\right|_{Y}} \in L^{\infty}\left(Y, \mathcal{A}^{\prime}, \mu^{\prime}\right)$. Claramente temos $\phi\left(\left[g_{\left.\right|_{Y}}\right]_{\mu^{\prime}}\right)=\left[\overline{g_{\left.\right|_{Y}}}\right]_{\mu}=[g]_{\mu}=[f]_{\mu}$, ou seja, $[f]_{\mu} \in \operatorname{Im}(\phi)$. Isso mostra que $\mathcal{B} \subset \operatorname{Im}(\phi)$, e conseqüentemente, $\mathcal{B}=\operatorname{Im}(\phi)$. Por fim, uma vez que $\phi$ é linear, segue que $\mathcal{B}$ é um subespaço vetorial de $L^{p}(X, \mathcal{A}, \mu)$.

As proposições 1.4 .80 e 1.4.81 nos dizem que para qualquer $p \in[1,+\infty]$, o espaço $L^{p}\left(Y, \mathcal{A}^{\prime}, \mu^{\prime}\right)$ pode ser identificado isometricamente com o subespaço de $L^{p}(X, \mathcal{A}, \mu)$ consistindo das classes de aplicações que admitem um representante que se anula fora de $Y$.

ObSERVAÇÃo 1.4.82. Se $(X, \mathcal{A}, \mu)$ é um espaço de medida arbitrário e $Y$ é um subconjunto mensurável de $X$ tal que $\mu(X \backslash Y)=0$ então, a 
menos de identificação isométrica, $L^{p}\left(Y,\left.\mathcal{A}\right|_{Y},\left.\mu\right|_{\left.\mathcal{A}\right|_{Y}}\right)=L^{p}(X, \mathcal{A}, \mu)$ para todo $p \in[1,+\infty]$. Com efeito, se $\mathcal{B}$ é a imagem da aplicação 1.4.1 então, pela Proposição 1.4.81, $L^{p}\left(Y,\left.\mathcal{A}\right|_{Y},\left.\mu\right|_{\left.\mathcal{A}\right|_{Y}}\right) \cong \mathcal{B} \subset L^{p}(X, \mathcal{A}, \mu)$. Por outro lado, para cada $[f]_{\mu} \in L^{p}(X, \mathcal{A}, \mu)$, a função $f \chi_{Y}: X \rightarrow \mathbb{R}$ se anula fora de $Y$ e $f \chi_{Y}=f \mu$-qs, uma vez que $\mu(X \backslash Y)=0$. Assim, $[f]_{\mu}=\left[f \chi_{Y}\right]_{\mu} \in \mathcal{B}$, ou seja, $L^{p}(X, \mathcal{A}, \mu) \subset \mathcal{B}$, e portanto $L^{p}\left(Y,\left.\mathcal{A}\right|_{Y},\left.\mu\right|_{\left.\mathcal{A}\right|_{Y}}\right) \cong \mathcal{B}=L^{p}(X, \mathcal{A}, \mu)$.

A seguir enunciamos o resultado que será nosso objeto de estudo: o conhecido Teorema de Representação de Riesz. A grosso modo, ele afirma que para $p, q \in] 1,+\infty\left[\right.$ conjugados, $L^{q}$ é o dual topológico de $L^{p}$ para todo espaço de medida, e que $L^{\infty}$ é o dual de $L^{1}$ para espaços de medida $\sigma$-finita.

Teorema 1.4.83 (de Representação de Riesz). Seja $(X, \mathcal{A}, \mu)$ um espaço de medida $\sigma$-finita. Sejam $p \in[1,+\infty]$ e $q$ dado por $1 / p+1 / q=1$. Se $g \in L^{q}(X, \mathcal{A}, \mu)$ então a função $\alpha_{g}: L^{p}(X, \mathcal{A}, \mu) \rightarrow \mathbb{R}$, definida por

$$
\alpha_{g}(f)=\int_{X} f g \mathrm{~d} \mu, \quad f \in L^{p}(X, \mathcal{A}, \mu)
$$

é um funcional linear limitado tal que $\left\|\alpha_{g}\right\|=\|g\|_{q}$. Reciprocamente, se $\alpha$ é um funcional linear limitado sobre $L^{p}(X, \mathcal{A}, \mu), p \in[1,+\infty[$, então existe $g \in L^{q}(X, \mathcal{A}, \mu)$ tal que $\alpha(f)=\int_{X} f g \mathrm{~d} \mu$ para cada $f \in L^{p}(X, \mathcal{A}, \mu)$.

Demonstração. Vide [8].

Teorema 1.4.84 (de Representação de Riesz). Se $p \in] 1,+\infty[$ então $a$ hipótese de que a medida $\mu$ seja $\sigma$-finita pode ser suprimida.

Demonstração. Vide [8].

Em outras palavras, o Teorema de Representação de Riesz afirma que para um espaço de medida arbitrário $(X, \mathcal{A}, \mu)$ e $p, q \in] 1,+\infty[$ tais que $\frac{1}{p}+\frac{1}{q}=1$, a $(q, p)$-aplicação de Riesz

$$
L^{q}(X, \mathcal{A}, \mu) \ni g \longmapsto \alpha_{g} \in L^{p}(X, \mathcal{A}, \mu)^{*},
$$

em que

$$
\alpha_{g}(f)=\int_{X} f g \mathrm{~d} \mu, \quad f \in L^{p}(X, \mathcal{A}, \mu),
$$


é uma isometria linear. E se $(X, \mathcal{A}, \mu)$ é um espaço de medida $\sigma$-finita então a aplicação de Riesz

$$
L^{\infty}(X, \mathcal{A}, \mu) \ni g \longmapsto \alpha_{g} \in L^{1}(X, \mathcal{A}, \mu)^{*},
$$

com $\alpha_{g}$ definido como em (1.4.3), também é uma isometria linear.

ObSERVAÇÃo 1.4.85. Sejam $(X, \mathcal{A}, \mu)$ um espaço de medida e $Y$ um subconjunto mensurável de $X$. Sejam $\mathcal{A}^{\prime}$ e $\mu^{\prime}$, respectivamente, a $\sigma$-álgebra induzida por $\mathcal{A}$ em $Y$ e a restrição da medida $\mu$ a $\mathcal{A}^{\prime}$, i.e., $\mathcal{A}^{\prime}=\left.\mathcal{A}\right|_{Y}$ e $\mu^{\prime}=\left.\mu\right|_{\mathcal{A}^{\prime}}$. Sejam $\alpha$ e $\beta$ as aplicações de Riesz (1.4.4) para os espaços $(X, \mathcal{A}, \mu)$ e $\left(Y, \mathcal{A}^{\prime}, \mu^{\prime}\right)$ respectivamente. Então é comutativo o diagrama:

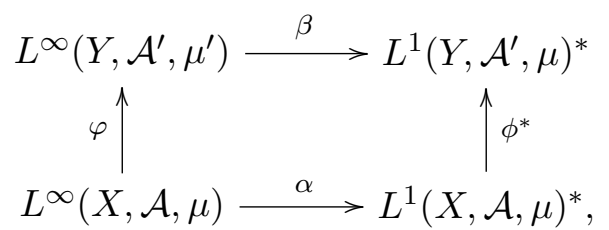

no qual $\phi^{*}$ é a transposta da aplicação (1.4.1) e $\varphi$ é a aplicação que leva $[g]_{\mu}$ em $\left[g_{\left.\right|_{Y}}\right]_{\mu^{\prime}}$ para toda $g \in L^{\infty}(X, \mathcal{A}, \mu)$. De fato, se $g \in L^{\infty}(X, \mathcal{A}, \mu)$ então, para toda $f \in L^{1}(X, \mathcal{A}, \mu)$ temos

$$
\begin{gathered}
(\beta \circ \varphi)(g)\left([f]_{\mu^{\prime}}\right)=\beta_{g_{\left.\right|_{Y}}}\left([f]_{\mu^{\prime}}\right)=\int_{Y} f g_{\left.\right|_{Y}} \mathrm{~d} \mu^{\prime} \text { e } \\
\phi^{*}(\alpha(g))\left([f]_{\mu^{\prime}}\right)=\alpha_{g}\left(\phi\left([f]_{\mu^{\prime}}\right)\right)=\alpha_{g}\left([\bar{f}]_{\mu}\right)=\int_{Y} \bar{f} g \mathrm{~d} \mu=\int_{Y} f g_{\left.\right|_{Y}} \mathrm{~d} \mu^{\prime},
\end{gathered}
$$

pois $\left.\bar{f}\right|_{Y}=f$ e $\left.\bar{f}\right|_{Y^{c}}=0$. Portanto o diagrama comuta, e assim, a menos de identificação, $\beta_{g_{\mid}}$é igual à restrição de $\alpha_{g}$ ao espaço $L^{1}\left(Y, \mathcal{A}^{\prime}, \mu^{\prime}\right)$.

Lema 1.4.86. Dados espaços de medida $(X, \mathcal{A}, \mu),\left(X^{\prime}, \mathcal{A}^{\prime}, \mu^{\prime}\right)$ e uma aplicação $\phi: X \rightarrow X^{\prime}$ que preserva medida então, para todo $p \in[1,+\infty]$, a aplicação induzida por $\phi$ em $L^{p}$,

$$
L^{p}\left(X^{\prime}, \mathcal{A}^{\prime}, \mu^{\prime}\right) \ni f \longmapsto f \circ \phi \in L^{p}(X, \mathcal{A}, \mu),
$$

é uma imersão isométrica. Além disso, se $\phi$ é uma aplicação quociente e $\mu$ é completa então a imagem de (1.4.5) consiste das aplicações $g \in L^{p}(X, \mathcal{A}, \mu)$ para as quais existe uma função $f: X^{\prime} \rightarrow \mathbb{R}$ tal que $f \circ \phi=g \mu$-qs. 
Demonstração. Seja $\varphi$ a aplicação (1.4.5). Vamos mostrar inicialmente que $\varphi$ é uma imersão isométrica. Seja $f: X^{\prime} \rightarrow \mathbb{R}$ uma função mensurável arbitrária. Como $\phi$ preserva medida, $\phi$ é mensurável; logo, pela Observação 1.4.33, a aplicação $f \circ \phi$ também é mensurável. Afirmamos que $\|f \circ \phi\|_{p}=\|f\|_{p}$ para todo $p \in[1,+\infty]$. De fato, se $1 \leq p<+\infty$ então, pela Proposição 1.4.66,

$$
\|f \circ \phi\|_{p}=\left(\int_{X}|f \circ \phi|^{p} \mathrm{~d} \mu\right)^{\frac{1}{p}}=\left(\int_{X^{\prime}}|f|^{p} \mathrm{~d} \mu^{\prime}\right)^{\frac{1}{p}}=\|f\|_{p} .
$$

Para cada número real não negativo $c$, sejam

$$
A=\{x \in X:|(f \circ \phi)(x)|>c\} \quad \text { e } \quad B=\left\{y \in X^{\prime}:|f(y)|>c\right\} .
$$

Como $f \circ \phi$ é $\mathcal{A}$-mensurável e $f$ é $\mathcal{A}^{\prime}$-mensurável temos que $A \in \mathcal{A}$ e $B \in \mathcal{A}^{\prime}$, e portanto $\phi^{-1}(B) \in \mathcal{A}$. Note que se $x \in A$ então $|(f \circ \phi)(x)|>c$; logo $\phi(x) \in B$, ou seja, $x \in \phi^{-1}(B)$. Por outro lado, se $x \in \phi^{-1}(B)$ então $\phi(x) \in B$, e daí $|(f \circ \phi)(x)|=|f(\phi(x))|>c$, i.e., $x \in A$. Isso mostra que $A=\phi^{-1}(B)$, e assim $\mu(A)=\mu\left(\phi^{-1}(B)\right)=\mu^{\prime}(B)$. Logo, $\mu(A)=0$ se e somente se $\mu^{\prime}(B)=0$, ou seja, $|(f \circ \phi)(x)| \leq c \mu$-qs se e somente se $|f| \leq c$ $\mu^{\prime}$-qs. Portanto,

$$
\begin{aligned}
\|f \circ \phi\|_{\infty}=\inf \{c \geq 0: \mid & (f \circ \phi)(x) \mid \leq c \mu-\mathrm{qs}\} \\
& =\inf \left\{c \geq 0:|f| \leq c \mu^{\prime}-\mathrm{qs}\right\}=\|f\|_{\infty} .
\end{aligned}
$$

A aplicação $\varphi$ é claramente linear, e está bem definida. Com efeito, sejam $f, g \in L^{p}\left(X^{\prime}, \mathcal{A}^{\prime}, \mu^{\prime}\right)$ com $f=g \mu^{\prime}$-qs, e consideremos os conjuntos

$$
E=\{x \in X:(f \circ \phi)(x) \neq(g \circ \phi)(x)\} \text { e } F=\left\{y \in X^{\prime}: f(y) \neq g(y)\right\} .
$$

De modo análogo ao que foi feito acima, podemos mostrar que $E=\phi^{-1}(F)$, e portanto $\mu(E)=0$ se e somente se $\mu^{\prime}(F)=0$. Assim, se $f=g \mu^{\prime}$-qs então $f \circ \phi=g \circ \phi \mu$-qs, o que mostra que a imagem da classe $[f]_{\mu^{\prime}}$ pela aplicação $\varphi$ independe do representante tomado. Além disso, se $f$ está em $L^{p}\left(X^{\prime}, \mathcal{A}^{\prime}, \mu^{\prime}\right)$ então $\|f \circ \phi\|_{p}=\|f\|_{p}<+\infty$, i.e., $f \circ \phi \in L^{p}(X, \mathcal{A}, \mu)$. Portanto $\varphi$ está bem definida e é uma imersão isométrica para todo $p \in[1+\infty]$. Suponhamos agora que $\phi$ é uma aplicação quociente e que a medida $\mu$ é completa. Seja $\mathcal{I}$ o conjunto das aplicações $g \in L^{p}(X, \mathcal{A}, \mu)$ para as quais existe uma função $f: X^{\prime} \rightarrow \mathbb{R}$ tal que $f \circ \phi=g \mu$-qs. Queremos mostrar que $\mathcal{I}=\operatorname{Im} \varphi$. É claro que se $g \in \operatorname{Im} \varphi$ então existe $f \in L^{p}\left(X^{\prime}, \mathcal{A}^{\prime}, \mu^{\prime}\right)$ tal que $f \circ \phi=\varphi(f)=g$, 
e portanto $\operatorname{Im} \varphi \subset \mathcal{I}$. Por outro lado, se $g \in \mathcal{I}$ então existe uma função $f: X^{\prime} \rightarrow \mathbb{R}$ tal que $f \circ \phi=g \mu$-qs. Como $\mu$ é completa e $g$ é $\mathcal{A}$-mensurável temos, pela Proposição 1.4.41, que $f \circ \phi$ é $\mathcal{A}$-mensurável, e uma vez que $\phi$ é uma aplicação quociente, a Observação 1.4 .49 garante a $\mathcal{A}^{\prime}$-mensurabilidade da função $f$. Ainda, $\|f\|_{p}=\|f \circ \phi\|_{p}=\|g\|_{p}<+\infty$, i.e., $f \in L^{p}\left(X^{\prime}, \mathcal{A}^{\prime}, \mu^{\prime}\right)$; portanto $g \in \operatorname{Im} \varphi$. Assim, $\mathcal{I} \subset \operatorname{Im} \varphi$, e conseqüentemente $\mathcal{I}=\operatorname{Im} \varphi$.

Corolário 1.4.87. Se $(X, \mathcal{A}, \mu)$ e $\left(X^{\prime}, \mathcal{A}^{\prime}, \mu^{\prime}\right)$ são espaços de medida e $\phi: X \rightarrow X^{\prime}$ é um isomorfismo então, para todo $p \in[1,+\infty]$, a aplicação (1.4.5) é uma isometria linear.

DemonstraçÃo. Sejam $p \in[1,+\infty]$ e $\varphi$ a aplicação (1.4.5). Pelo Lema 1.4.86, $\varphi$ é uma imersão isométrica e dada $g \in L^{p}(X, \mathcal{A}, \mu)$, a aplicação $g \circ \phi^{-1}$ está em $L^{p}\left(X^{\prime}, \mathcal{A}^{\prime}, \mu^{\prime}\right)$ e $\varphi\left(g \circ \phi^{-1}\right)=g$; isso mostra que $\varphi$ é sobrejetora, e portanto uma isometria linear.

Dados $p, q \in[1,+\infty]$ com $\frac{1}{p}+\frac{1}{q}=1$, espaços de medida $(X, \mathcal{A}, \mu)$, $\left(X^{\prime}, \mathcal{A}^{\prime}, \mu^{\prime}\right)$ e uma aplicação $\phi: X \rightarrow X^{\prime}$ que preserva medida temos que é comutativo o diagrama:

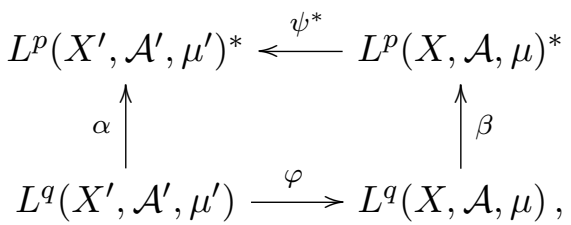

no qual $\alpha$ e $\beta$ são $(q, p)$-aplicações de Riesz (1.4.2) para os espaços $\left(X^{\prime}, \mathcal{A}^{\prime}, \mu^{\prime}\right)$ e $(X, \mathcal{A}, \mu), \psi^{*}$ é a transposta da aplicação induzida por $\phi$ em $L^{p}$ e $\varphi$ é aplicação induzida por $\phi$ em $L^{q}$. De fato, para cada $g \in L^{q}\left(X^{\prime}, \mathcal{A}^{\prime}, \mu^{\prime}\right)$ temos que $\alpha(g)=\alpha_{g} \in L^{p}\left(X^{\prime}, \mathcal{A}^{\prime}, \mu^{\prime}\right)^{*}$, tal que $\alpha_{g}(f)=\int_{X^{\prime}} f g \mathrm{~d} \mu^{\prime}$ para toda $f \in L^{p}\left(X^{\prime}, \mathcal{A}^{\prime}, \mu^{\prime}\right)$. Por outro lado, $\varphi$ leva $g$ em $g \circ \phi \in L^{q}(X, \mathcal{A}, \mu)$, e $\beta(g \circ \phi)=\beta_{g \circ \phi} \in L^{p}(X, \mathcal{A}, \mu)^{*}$, tal que $\beta_{g \circ \phi}(h)=\int_{X} h(g \circ \phi) \mathrm{d} \mu$ para toda $h \in L^{p}(X, \mathcal{A}, \mu)$. Ainda, para cada $f \in L^{p}\left(X^{\prime}, \mathcal{A}^{\prime}, \mu^{\prime}\right)$ temos

$$
\begin{aligned}
& \psi^{*}\left(\beta_{g \circ \phi}\right)(f)=\beta_{g \circ \phi}(\psi(f))=\beta_{g \circ \phi}(f \circ \phi) \\
&=\int_{X}(f \circ \phi)(g \circ \phi) \mathrm{d} \mu=\int_{X}((f g) \circ \phi) \mathrm{d} \mu=\int_{X^{\prime}} f g \mathrm{~d} \mu^{\prime},
\end{aligned}
$$

sendo a última igualdade garantida pela Proposição 1.4.66, visto que $f g$ é integrável. Assim, $\left(\left(\psi^{*} \circ \beta \circ \varphi\right)(g)\right)(f)=(\alpha(g))(f)$ para cada $f$ em 
$L^{p}\left(X^{\prime}, \mathcal{A}^{\prime}, \mu^{\prime}\right)$ e para cada $g$ em $L^{q}\left(X^{\prime}, \mathcal{A}^{\prime}, \mu^{\prime}\right)$. Portanto $\psi^{*} \circ \beta \circ \varphi=\alpha$, o que mostra que o diagrama (1.4.6) comuta.

Lema 1.4.88. Sejam $(X, \mathcal{A}, \mu)$ e $\left(X^{\prime}, \mathcal{A}^{\prime}, \mu^{\prime}\right)$ espaços de medida. Se $\phi: X \rightarrow X^{\prime}$ é uma aplicação quociente então $\phi(X)$ é um subconjunto mensurável de $X^{\prime}$ cujo complemento tem medida nula. Além disso, se $\sim$ é a relação de equivalência em $X$ induzida por $\phi$

$$
\text { (i.e., } x \sim y \text { se e somente se } \phi(x)=\phi(y), \quad x, y \in X),
$$

e o conjunto quociente $X / \sim$ está munido com a $\sigma$-álgebra e medida coinduzidas pela aplicação canônica $\mathfrak{q}: X \rightarrow X / \sim$, então $\phi$ induz um isomorfismo $\bar{\phi}:(X / \sim) \rightarrow \phi(X)$ tal que é comutativo o diagrama:

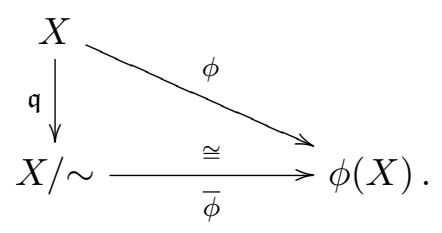

Demonstração. Se $\phi$ é uma aplicação quociente então

$$
\mathcal{A}^{\prime}=\phi_{*} \mathcal{A}=\left\{A \subset X^{\prime}: \phi^{-1}(A) \in \mathcal{A}\right\}
$$

e $\mu^{\prime}(A)=\left(\phi_{*} \mu\right)(A)=\mu\left(\phi^{-1}(A)\right)$ para todo $A \in \mathcal{A}^{\prime}$. Assim, uma vez que $\phi^{-1}(\phi(X))=X \in \mathcal{A}$, o conjunto $\phi(X)$ é $\mathcal{A}^{\prime}$-mensurável. Além disso,

$$
\mu^{\prime}\left(X^{\prime} \backslash \phi(X)\right)=\mu\left(\phi^{-1}\left(X^{\prime} \backslash \phi(X)\right)\right)=\mu(\emptyset)=0 .
$$

Seja $\bar{\phi}:(X / \sim) \rightarrow \phi(X)$ definida por $\bar{\phi}([x])=\phi(x)$, para todo $x \in X$. Do enunciado temos o diagrama

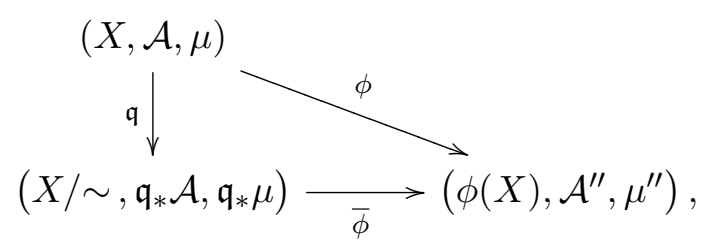

no qual $\mathcal{A}^{\prime \prime}=\left.\mathcal{A}^{\prime}\right|_{\phi(X)}=\mathcal{P}(\phi(X)) \cap \mathcal{A}^{\prime} \subset \mathcal{A}^{\prime}, \mu^{\prime \prime}=\left.\mu^{\prime}\right|_{\mathcal{A}^{\prime \prime}}$, e $\mathfrak{q}_{*} \mathcal{A}$ e $\mathfrak{q}_{*} \mu$ são respectivamente a $\sigma$-álgebra e a medida co-induzidas por $\mathfrak{q}$ em $X / \sim$. Para todo $x \in X$ temos

$$
(\bar{\phi} \circ \mathfrak{q})(x)=\bar{\phi}(\mathfrak{q}(x))=\bar{\phi}([x])=\phi(x)
$$


$\operatorname{logo} \bar{\phi} \circ \mathfrak{q}=\phi . \quad$ Sejam $x, y \in X$ arbitrários. Então $\bar{\phi}([x])=\bar{\phi}([y])$ se e somente se $\phi(x)=\phi(y)$, o que equivale a $x \sim y$, i.e., $[x]=[y]$. Isso mostra que $\bar{\phi}$ é injetora e que a imagem da classe $[x]$ por $\bar{\phi}$ independe do representante tomado. É claro que se $y \in \phi(X)$ então existe $x \in X$ tal que $y=\phi(x)$, e daí $\bar{\phi}([x])=\phi(x)=y$, ou seja, $\bar{\phi}$ é sobrejetora. Além disso,

$$
\bar{\phi}_{*}\left(\mathfrak{q}_{*} \mathcal{A}\right)=\left\{A \subset \phi(X):(\bar{\phi})^{-1}(A) \in \mathfrak{q}_{*} \mathcal{A}\right\}
$$

$\mathrm{e}(\bar{\phi})^{-1}(A) \in \mathfrak{q}_{*} \mathcal{A}$ se e somente se

$$
\mathfrak{q}^{-1}\left((\bar{\phi})^{-1}(A)\right)=(\bar{\phi} \circ \mathfrak{q})^{-1}(A)=\phi^{-1}(A) \in \mathcal{A} .
$$

Portanto $\bar{\phi}_{*}\left(\mathfrak{q}_{*} \mathcal{A}\right)=\left\{A \in \phi(X): \phi^{-1}(A) \in \mathcal{A}\right\}=\left.\left(\phi_{*} \mathcal{A}\right)\right|_{\phi(X)}=\mathcal{A}^{\prime \prime}$. Por fim, para todo $A \in \phi(X)$, temos que

$$
\begin{aligned}
\mu^{\prime \prime}(A)=\mu^{\prime}(A) & =\mu\left(\phi^{-1}(A)\right)=\mu\left((\bar{\phi} \circ \mathfrak{q})^{-1}(A)\right) \\
= & \mu\left(\mathfrak{q}^{-1}\left((\bar{\phi})^{-1}(A)\right)\right)=\left(\mathfrak{q}_{*} \mu\right)\left((\bar{\phi})^{-1}(A)\right)=\left(\bar{\phi}_{*}\left(\mathfrak{q}_{*} \mu\right)\right)(A),
\end{aligned}
$$

isto é, $\mu^{\prime \prime}=\bar{\phi}_{*}\left(\mathfrak{q}_{*} \mu\right)$. Assim, pelo que vimos acima, o diagrama é comutativo e $\bar{\phi}$ é uma aplicação quociente bijetora, portanto um isomorfismo. 


\section{CAPíTULO 2}

\section{Espaços de medida perfeita}

Neste capítulo estudamos modificações que se realizadas em um espaço de medida $(X, \mathcal{A}, \mu)$ arbitrário "melhoram" as chances de bijetividade da aplicação de Riesz (1.4.4).

\subsection{Medidas livres de blocos}

Definição 2.1.1. Seja $(X, \mathcal{A}, \mu)$ um espaço de medida. Um subconjunto mensurável $B \in \mathcal{A}$ é chamado de um bloco infinito para $\mu$ se $\mu(B)=+\infty$ e $\mu(A) \in\{0,+\infty\}$, para cada $A \in \mathcal{A}$ contido em $B$. Se não existem blocos infinitos para $\mu$ então $\mu$ é chamada de uma medida livre de blocos.

ObservaÇão 2.1.2. Dado um espaço de medida $(X, \mathcal{A}, \mu)$, se $B \in \mathcal{A}$ é um bloco infinito para $\mu$ então diremos alternativamente que $B$ é um $\mu$-bloco infinito ou apenas um bloco infinito, caso não exista risco de confusão.

Lema 2.1.3. Seja $(X, \mathcal{A}, \mu)$ um espaço de medida. Se $A \subset X$ é um subconjunto $\sigma$-finito para $\mu$, e $B \in \mathcal{A}$ é um bloco infinito então $\mu(A \cap B)=0$.

Demonstração. Se $A$ é $\sigma$-finito para $\mu$ então existe uma seqüência $\left(A_{n}\right)_{n \geq 1}$ de conjuntos $\mathcal{A}$-mensuráveis com $\mu\left(A_{n}\right)<+\infty$ para todo $n \geq 1$

e $A=\bigcup_{n \geq 1} A_{n}$. Para cada $n \geq 1$ temos $\mu\left(A_{n} \cap B\right) \leq \mu\left(A_{n}\right)<+\infty$, e portanto $\mu\left(A_{n} \cap B\right)=0$, uma vez que $B$ é um bloco infinito. Assim,

$$
\mu(A \cap B)=\mu\left(\left(\bigcup_{n \geq 1} A_{n}\right) \cap B\right)=\mu\left(\bigcup_{n \geq 1}\left(A_{n} \cap B\right)\right) \leq \sum_{n \geq 1} \mu\left(A_{n} \cap B\right)=0,
$$

ou seja, $\mu(A \cap B)=0$.

Lema 2.1.4. Sejam $(X, \mathcal{A}, \mu)$ um espaço de medida e $f \in L^{p}(X, \mathcal{A}, \mu)$, com $p \in\left[1,+\infty\left[\right.\right.$. Então o conjunto $f^{-1}(\mathbb{R} \backslash\{0\})$ é $\sigma$-finito para $\mu$ e $\left.f\right|_{B}=0$ $\mu$-qs para todo $\mu$-bloco infinito $B$ 
DemonstraÇÃo. Se $p \in\left[1,+\infty\left[\right.\right.$ e $f \in L^{p}(X, \mathcal{A}, \mu)$ então, para cada $n \geq 1$, o conjunto $A_{n} \stackrel{\text { def }}{=}\left\{x \in X:|f(x)| \geq \frac{1}{n}\right\}$ é mensurável e

$$
\left(\frac{1}{n}\right)^{p} \mu\left(A_{n}\right)=\int_{A_{n}}\left(\frac{1}{n}\right)^{p} \mathrm{~d} \mu \leq \int_{A_{n}}|f|^{p} \mathrm{~d} \mu \leq \int_{X}|f|^{p} \mathrm{~d} \mu<+\infty,
$$

ou seja, $\mu\left(A_{n}\right)<+\infty$ para todo $n \geq 1$. Assim, uma vez que

$$
f^{-1}(\mathbb{R} \backslash\{0\})=\{x \in X:|f(x)|>0\}=\bigcup_{n \geq 1}\left\{x \in X:|f(x)| \geq \frac{1}{n}\right\}=\bigcup_{n \geq 1} A_{n},
$$

o conjunto $f^{-1}(\mathbb{R} \backslash\{0\})$ é $\sigma$-finito para $\mu$. Se $B$ é um $\mu$-bloco infinito então, pelo Lema 2.1.3, $\mu\left(f^{-1}(\mathbb{R} \backslash\{0\}) \cap B\right)=0$, i.e., $\mu(\{x \in B: f(x) \neq 0\})=0$, e isso mostra que $\left.f\right|_{B}=0 \mu$-qs.

Proposição 2.1.5. A aplicação de Riesz (1.4.4) é injetora se e somente se $\mu$ é livre de blocos; nesse caso, (1.4.4) é uma imersão isométrica.

DemonstraÇÃo. Vamos mostrar inicialmente que a injetividade da aplicação de Riesz (1.4.4) implica em $\mu$ ser uma medida livre de blocos. Suponhamos que $\mu$ não seja uma medida livre de blocos, isto é, existe um bloco infinito $B \subset X$. Sejam $g=\chi_{B} \neq 0$ e $f \in L^{1}(X, \mathcal{A}, \mu)$ arbitrária. Então $g$ é mensurável e limitada, ou seja, $g \in L^{\infty}(X, \mathcal{A}, \mu)$, e

$$
\alpha_{g}(f)=\int_{X} f g \mathrm{~d} \mu=\int_{X} f \chi_{B} \mathrm{~d} \mu=\int_{B} f \mathrm{~d} \mu=0,
$$

pois $\left.f\right|_{B}=0 \mu$-qs, pelo Lema 2.1.4. Logo $g$ pertence ao núcleo de (1.4.4), o que significa que (1.4.4) não é injetora. Suponhamos agora que $\mu$ seja livre de blocos. Dada $g \in L^{\infty}(X, \mathcal{A}, \mu)$, sejam $E$ e $F$ os conjuntos:

$$
\begin{gathered}
E=\{c \in[0,+\infty]:|g| \leq c \mu \text {-qs }\} \text { e } \\
F=\left\{c \in[0,+\infty]:\left|\alpha_{g}(f)\right| \leq c\|f\|_{1}, \text { para toda } f \in L^{1}(X, \mathcal{A}, \mu)\right\} .
\end{gathered}
$$

Queremos mostrar que $E=F$, e daí seguirá que

$$
\|g\|_{\infty}=\inf E=\inf F=\left\|\alpha_{g}\right\|
$$

para toda $g \in L^{\infty}(X, \mathcal{A}, \mu)$, ou seja, que (1.4.4) é uma imersão isométrica, e portanto injetora. Com efeito, se $c \in E$ então $|g| \leq c \mu$-qs, e assim,

$$
\left|\alpha_{g}(f)\right|=\left|\int_{X} f g \mathrm{~d} \mu\right| \leq \int_{X}|f||g| \mathrm{d} \mu \leq \int_{X} c|f| \mathrm{d} \mu=c\|f\|_{1},
$$

para cada $f \in L^{1}(X, \mathcal{A}, \mu)$. Logo $c \in F$, e portanto $E \subset F$. Por outro lado, se $c \notin E$ então não é verdade que $|g| \leq c \mu$-qs, i.e., se $B$ é o conjunto 
$\{x \in X:|g(x)|>c\}$ então $\mu(B)>0$. Afirmamos que existe $A \subset B$ mensurável tal que $0<\mu(A)<+\infty$. De fato, se $\mu(B)<+\infty$, basta tomar $A=B$, senão existe $A \subset B \mathcal{A}$-mensurável com $0<\mu(A)<+\infty$, uma vez que $B$ não é um bloco infinito. Seja $f=\operatorname{sgn}(g) \chi_{A}$; uma vez que $g$ é uma função mensurável e $A \in \mathcal{A}$, a aplicação $f$ também é mensurável e

$$
\int_{X}|f| \mathrm{d} \mu=\int_{X}\left|\operatorname{sgn}(g) \chi_{A}\right| \mathrm{d} \mu=\int_{A}|\operatorname{sgn}(g)| \mathrm{d} \mu=\int_{A} 1 \mathrm{~d} \mu=\mu(A)<+\infty,
$$

i.e., $f \in L^{1}(X, \mathcal{A}, \mu)$. Como $A \subset B=\{x \in X:|g(x)|>c\}$ e $\mu(A)>0$ temos $\left.g\right|_{A} \neq 0$, e conseqüentemente $f \neq 0$. Além disso,

$$
\begin{aligned}
\left|\alpha_{g}(f)\right| \geq \alpha_{g}(f) & =\int_{X} f g \mathrm{~d} \mu=\int_{X}\left(\left(\operatorname{sgn}(g) \chi_{A}\right) g\right) \mathrm{d} \mu=\int_{X}|g| \chi_{A} \mathrm{~d} \mu \\
= & \int_{A}|g| \mathrm{d} \mu>\int_{A} c \mathrm{~d} \mu=c \int_{X} \chi_{A} \mathrm{~d} \mu=c \int_{X}|f| \mathrm{d} \mu=c\|f\|_{1} .
\end{aligned}
$$

Logo $c \notin F$, e portanto $F \subset E$.

Em alguns espaços de medida $(X, \mathcal{A}, \mu)$ podemos "isolar" os blocos infinitos, isto é, podemos escrever $X$ como uma união disjunta $X=X_{\infty} \cup X_{0}$, na qual $X_{\infty}$ é um bloco infinito e $X_{0}$ é um conjunto que não contém blocos infinitos. Veremos no Exemplo 2.1.6 que isso nem sempre é possível, ou seja, existem espaços de medida que não admitem tal decomposição. Para o exemplo a seguir, lembramos que $\mathcal{L}$ denota a coleção dos conjuntos Lebesgue mensuráveis de $\mathbb{R}$, e $\mathfrak{m}$ denota a medida de Lebesgue na reta.

Exemplo 2.1.6. Seja $X$ o retângulo $[0,1]^{2}$. Para todo $A \subset X$ e para cada $y \in[0,1], A^{y}$ denotará a linha $\{x \in[0,1]:(x, y) \in A\}$. O conjunto

$$
\mathcal{A}=\left\{A \subset X: A^{y} \in \mathcal{L} \text { para todo } y \in[0,1]\right\}
$$

é uma $\sigma$-álgebra de partes de $X$. De fato, para cada $y \in[0,1]$, considere a aplicação $i^{y}:[0,1] \rightarrow[0,1]^{2}$ definida por $i^{y}(x)=(x, y)$ para todo $x \in[0,1]$. Então $A^{y}=\{x \in[0,1]:(x, y) \in A\}=\left(i^{y}\right)^{-1}(A)$ para cada $y \in[0,1]$, e portanto:

$$
\mathcal{A}=\left\{A \subset X:\left(i^{y}\right)^{-1}(A) \in \mathcal{L} \text { para todo } y \in[0,1]\right\}
$$


Pela Proposição 1.4.11, $\mathcal{A}$ é a $\sigma$-álgebra co-induzida em $X$ pela família de funções $\left(i^{y}\right)_{y \in[0,1]}$. Seja $\mu: \mathcal{A} \rightarrow[0,+\infty]$ a aplicação definida por

$$
\mu(A)=\sum_{y \in[0,1]} \mathfrak{m}\left(A^{y}\right)
$$

se o conjunto $\left\{y \in[0,1]: A^{y} \neq \emptyset\right\}$ é enumerável, e $\mu(A)=+\infty$ caso contrário, para todo $A \in \mathcal{A}$. A aplicação $\mu$ é uma medida em $\mathcal{A}$. Com efeito, para cada $y \in[0,1]$, temos que $\emptyset^{y}=\{x \in[0,1]:(x, y) \in \emptyset\}=\emptyset$; logo o conjunto $\left\{y \in[0,1]: \emptyset^{y} \neq \emptyset\right\}$ é vazio. Portanto,

$$
\mu(\emptyset)=\sum_{y \in[0,1]} \mathfrak{m}\left(\emptyset^{y}\right)=\sum_{y \in[0,1]} \mathfrak{m}(\emptyset)=0 .
$$

Seja $\left(A_{n}\right)_{n \geq 1}$ uma seqüência de elementos de $\mathcal{A}$, dois a dois disjuntos. Para todo $n \geq 1$ e $y \in[0,1]$ temos $A_{n}^{y} \in \mathcal{L}$. Observe ainda que, para cada $y \in[0,1]$,

$$
\begin{aligned}
\left(\bigcup_{n \geq 1} A_{n}\right)^{y} & =\left\{x \in[0,1]:(x, y) \in \bigcup_{n \geq 1} A_{n}\right\} \\
& =\bigcup_{n \geq 1}\left\{x \in[0,1]:(x, y) \in A_{n}\right\}=\bigcup_{n \geq 1} A_{n}^{y} .
\end{aligned}
$$

E portanto,

$$
\begin{aligned}
\left\{y \in[0,1]:\left(\bigcup_{n \geq 1} A_{n}\right)^{y} \neq \emptyset\right\} & =\left\{y \in[0,1]: \bigcup_{n \geq 1} A_{n}^{y} \neq \emptyset\right\} \\
& =\bigcup_{n \geq 1}\left\{y \in[0,1]: A_{n}^{y} \neq \emptyset\right\} .
\end{aligned}
$$

Se o conjunto $\left\{y \in[0,1]: A_{n}^{y} \neq \emptyset\right\}$ é enumerável para todo $n \geq 1$ então também é enumerável o conjunto $\left\{y \in[0,1]:\left(\bigcup_{n \geq 1} A_{n}\right)^{y} \neq \emptyset\right\}$. Assim,

$$
\begin{aligned}
\mu\left(\bigcup_{n \geq 1} A_{n}\right)=\sum_{y \in[0,1]} \mathfrak{m}\left(\left(\bigcup_{n \geq 1} A_{n}\right)^{y}\right) & =\sum_{y \in[0,1]} \mathfrak{m}\left(\bigcup_{n \geq 1} A_{n}^{y}\right) \\
= & \sum_{y \in[0,1]}\left(\sum_{n \geq 1} \mathfrak{m}\left(A_{n}^{y}\right)\right)=\sum_{n \geq 1}\left(\sum_{y \in[0,1]} \mathfrak{m}\left(A_{n}^{y}\right)\right)=\sum_{n \geq 1} \mu\left(A_{n}\right) .
\end{aligned}
$$

Suponhamos, por outro lado, que exista um número natural $k \geq 1$ tal que o conjunto $\mathfrak{B}=\left\{y \in[0,1]: A_{k}^{y} \neq \emptyset\right\}$ seja não enumerável. Visto que

$$
\mathfrak{B} \subset \bigcup_{n \geq 1}\left\{y \in[0,1]: A_{n}^{y} \neq \emptyset\right\}=\left\{y \in[0,1]:\left(\bigcup_{n \geq 1} A_{n}\right)^{y} \neq \emptyset\right\},
$$

o conjunto $\left\{y \in[0,1]:\left(\bigcup_{n \geq 1} A_{n}\right)^{y} \neq \emptyset\right\}$ também é não enumerável, e portanto $\mu\left(\bigcup_{n \geq 1} A_{n}\right)=+\infty$. Por outro lado, $\sum_{n \geq 1} \mu\left(A_{n}\right) \geq \mu\left(A_{k}\right)=+\infty$, 
i.e., $\sum_{n \geq 1} \mu\left(A_{n}\right)=+\infty$, o que mostra que $\mu\left(\bigcup_{n \geq 1} A_{n}\right)=\sum_{n \geq 1} \mu\left(A_{n}\right)$. Existem blocos infinitos em $X$; por exemplo, para cada $x \in[0,1]$, a coluna $B=\{x\} \times[0,1]$ é um bloco infinito. De fato, $\mu(B)=+\infty$, pois para cada $y \in[0,1]$ temos $B^{y}=\{x\}$, e assim o conjunto $\left\{y \in[0,1]: B^{y} \neq \emptyset\right\}=[0,1]$ é não enumerável; e se $A \subset B$ é um conjunto mensurável então

$$
\mu(A)=\sum_{y \in[0,1]} \mathfrak{m}\left(A^{y}\right)=\sum_{y \in[0,1]} \mathfrak{m}(\{x\})=0
$$

se o conjunto $\left\{y \in[0,1]: A^{y} \neq \emptyset\right\}$ é enumerável, e $\mu(A)=+\infty$ caso contrário. Afirmamos que o espaço $X$ não pode ser escrito como união disjunta $X=X_{0} \cup X_{\infty}$, em que $X_{\infty}$ é um bloco infinito e $X_{0}$ é um subconjunto de $X$ que não contém blocos infinitos para $\mu$. Para mostrar esse fato, verifiquemos preliminarmente que se $X_{\infty}$ é um bloco infinito então $\mathfrak{m}\left(X_{\infty}^{y}\right)=0$ para todo $y \in[0,1]$. Suponhamos, por absurdo, que exista $y_{0} \in[0,1]$ tal que $\mathfrak{m}\left(X_{\infty}^{y_{o}}\right) \neq 0$. Observe que

$$
\left(X_{\infty}^{y_{o}} \times\left\{y_{o}\right\}\right)^{y}= \begin{cases}X_{\infty}^{y_{o}} & \text { se } y=y_{o} \\ \emptyset & \text { se } y \neq y_{o}\end{cases}
$$

ou seja, $\left(X_{\infty}^{y_{o}} \times\left\{y_{o}\right\}\right)^{y} \in \mathcal{L}$ para todo $y \in[0,1]$, e portanto $X_{\infty}^{y_{o}} \times\left\{y_{o}\right\}$ é $\mathcal{A}$-mensurável. Ainda, como $\left\{y \in[0,1]:\left(X_{\infty}^{y_{o}} \times\left\{y_{o}\right\}\right)^{y} \neq \emptyset\right\}=\left\{y_{o}\right\}$, temos

$$
\begin{aligned}
\mu\left(X_{\infty}^{y_{o}} \times\left\{y_{o}\right\}\right) & =\sum_{y \in[0,1]} \mathfrak{m}\left(\left(X_{\infty}^{y_{o}} \times\left\{y_{o}\right\}\right)^{y}\right) \\
& =\mathfrak{m}\left(\left(X_{\infty}^{y_{o}} \times\left\{y_{o}\right\}\right)^{y_{o}}\right)=\mathfrak{m}\left(X_{\infty}^{y_{o}}\right) \leq 1,
\end{aligned}
$$

uma vez que $X_{\infty}^{y_{o}} \subset[0,1]$. Assim, o conjunto $X_{\infty}^{y_{o}} \times\left\{y_{o}\right\} \subset X_{\infty}$ tem medida finita não nula, o que contradiz o fato de $X_{\infty}$ ser um bloco infinito para $\mu$. Sendo $\mathfrak{m}\left(X_{\infty}^{y}\right)=0$ para todo $y \in[0,1]$, temos que para cada $y \in[0,1]$ existe um ponto $a(y) \in\left([0,1] \backslash X_{\infty}^{y}\right)$. O conjunto

$$
B=\{(a(y), y): y \in[0,1]\}
$$

é um bloco infinito para $\mu$ disjunto de $X_{\infty}$. De fato, para todo $y \in[0,1]$ temos $B^{y}=\{a(y)\} \in \mathcal{L}$, e portanto $\left\{y \in[0,1]: B^{y} \neq \emptyset\right\}=[0,1] ; \log 0$ $\mu(B)=+\infty$. Seja $A \subset B$ um conjunto $\mathcal{A}$-mensurável; se $A \subset B$ então existe um subconjunto $A^{\prime} \subset[0,1]$ tal que $A=\left\{(a(y), y): y \in A^{\prime}\right\}$. É claro 
que $\left\{y \in[0,1]: A^{y} \neq \emptyset\right\}=A^{\prime}$. Se o conjunto $A$ é enumerável então $A^{\prime}$ é enumerável e daí:

$$
\mu(A)=\sum_{y \in[0,1]} \mathfrak{m}\left(A^{y}\right)=\sum_{y \in A^{\prime}} \mathfrak{m}(\{a(y)\})=0 ;
$$

se $A$ é não enumerável então $A^{\prime}$ é não enumerável, logo $\mu(A)=+\infty$. Além disso, para cada $y \in[0,1]$ temos que $a(y) \notin X_{\infty}^{y}$, ou seja, $(a(y), y) \notin X_{\infty}$; assim $B \cap X_{\infty}=\emptyset$. Portanto, para todo bloco infinito $X_{\infty} \subset X$ tal que $X=X_{0} \cup X_{\infty}$ e $X_{0} \cap X_{\infty}=\emptyset$, existe um bloco infinito contido em $X_{0}$, ou seja, não é possível escrever $X$ como união disjunta de um bloco infinito de $X$ e um subconjunto de $X$ que não contém blocos infinitos.

Dado um espaço de medida $(X, \mathcal{A}, \mu)$, o Exemplo 2.1.6 mostra que tentar decompor $X$ de modo a "isolar" os blocos infinitos não é a maneira adequada para "livrar-se" deles. Vamos procurar "consertar" a medida $\mu$, definindo uma nova medida que seja uma espécie de "versão livre de blocos" de $\mu$. Seja $\mu_{\mathrm{lb}}: \mathcal{A} \rightarrow[0,+\infty]$ a aplicação definida por

$$
\mu_{\mathrm{lb}}(A)=\sup \{\mu(E): E \subset A, E \in \mathcal{A} \text { e } \mu(E)<+\infty\},
$$

para todo $A \in \mathcal{A}$. Não é difícil ver que, para cada $A \in \mathcal{A}, \mu_{\mathrm{lb}}(A) \leq \mu(A)$ (se $E \subset A$ e $E \in \mathcal{A}$ então $\mu(E) \leq \mu(A))$, e que $\mu_{\mathrm{lb}}(A)=0$ se e somente se $\mu(A)=0$ ou $A$ é um bloco infinito para $\mu$. Com efeito, se $\mu(A) \neq 0$ e $A$ não é um bloco infinito para $\mu$, então existe $B \subset A$ mensurável tal que $0<\mu(B)<+\infty$, e portanto $\mu_{\mathrm{lb}}(A) \geq \mu(B)>0$, ou seja, $\mu_{\mathrm{lb}}(A) \neq 0$. Por outro lado, se $\mu(A)=0$ então $\mu_{\mathrm{lb}}(A)=0$, visto que $\mu_{\mathrm{lb}}(A) \leq \mu(A)$; e se $A$ é um bloco infinito para $\mu$ então $\mu(E)=0$, para todo subconjunto $\mathcal{A}$-mensurável $E \subset A$ com $\mu(E)<+\infty, \operatorname{logo} \mu_{\mathrm{lb}}(A)=0$.

Proposição 2.1.7. Seja $(X, \mathcal{A}, \mu)$ um espaço de medida. A aplicação $\mu_{\mathrm{lb}}: \mathcal{A} \rightarrow[0,+\infty]$, definida como em (2.1.1), tem as seguintes propriedades:

(a) dado $A \in \mathcal{A}$, existe $E \subset A \sigma$-finito para $\mu$ tal que $\mu_{\mathrm{lb}}(A)=\mu(E)$;

(b) se $A \in \mathcal{A}$ não contém blocos infinitos para $\mu$ (em particular, se $A$ é $\sigma$-finito para $\mu$ ) então $\mu(A)=\mu_{\mathrm{lb}}(A)$;

(c) $\mu_{\mathrm{lb}}$ é uma medida livre de blocos;

(d) se $A \in \mathcal{A}$ é $\sigma$-finito para $\mu_{\mathrm{lb}}$ então $A$ pode ser escrito como união disjunta $A=A_{0} \cup A_{\infty}, A_{0}, A_{\infty} \in \mathcal{A}$, com $A_{0} \sigma$-finito para $\mu e$ $\mu_{\mathrm{lb}}\left(A_{\infty}\right)=0$ (e assim, ou $\mu\left(A_{\infty}\right)=0$ ou $A_{\infty}$ é um $\mu$-bloco infinito). 


\section{DEMONSTRAÇÃO.}

- Prova de (a).

Sejam $A \in \mathcal{A}$ e $\mathcal{B}=\{\mu(E): E \subset A, E \in \mathcal{A}$ e $\mu(E)<+\infty\}$. Como $\mu_{\mathrm{lb}}(A)=\sup \mathcal{B}$, existe uma seqüência $\left(E_{n}\right)_{n \geq 1}$ de subconjuntos mensuráveis de $A$ de medida finita tal que

$$
\lim _{n \rightarrow+\infty} \mu\left(E_{n}\right)=\mu_{\mathrm{lb}}(A) .
$$

Seja $E=\bigcup_{n \geq 1} E_{n}$. $E$ é claramente um subconjunto de $A \sigma$-finito para $\mu$. Vamos mostrar que $\mu(E)=\mu_{\mathrm{lb}}(A)$. Para cada $n \geq 1$, fazendo $F_{n}=\bigcup_{k=1}^{n} E_{k}$, temos que $\left(F_{n}\right)_{n \geq 1}$ é uma seqüência crescente de elementos de $\mathcal{A}$ tal que $\bigcup_{n \geq 1} F_{n}=\bigcup_{n \geq 1} E_{n}$. Desse modo, pelo item (a) da proposição 1.4 .25 , temos:

$$
\mu(E)=\mu\left(\bigcup_{n \geq 1} E_{n}\right)=\mu\left(\bigcup_{n \geq 1} F_{n}\right)=\lim _{n \rightarrow+\infty} \mu\left(F_{n}\right)
$$

Além disso, para cada $n \geq 1$,

$$
\mu\left(F_{n}\right)=\mu\left(\bigcup_{k=1}^{n} E_{k}\right) \leq \sum_{k=1}^{n} \mu\left(E_{k}\right)<+\infty
$$

e portanto $\mu\left(F_{n}\right) \leq \mu_{\mathrm{lb}}(A)$ para cada $n \geq 1$. Por outro lado, uma vez que $E_{n} \subset F_{n}$ para todo $n \geq 1$, temos $\mu\left(E_{n}\right) \leq \mu\left(F_{n}\right)$ para todo $n \geq 1$. Assim, $\mu\left(E_{n}\right) \leq \mu\left(F_{n}\right) \leq \mu_{\mathrm{lb}}(A)$ para todo $n \geq 1$. Fazendo $n \longrightarrow+\infty$ obtemos $\mu_{\mathrm{lb}}(A) \leq \mu(E) \leq \mu_{\mathrm{lb}}(A)$, i.e., $\mu(E)=\mu_{\mathrm{lb}}(A)$.

- Prova de (b).

Seja $A$ um conjunto mensurável que não contém blocos infinitos para $\mu$. Se $\mu_{\mathrm{lb}}(A)=+\infty$ então $\mu(A)=+\infty$, pois $\mu(A) \geq \mu_{\mathrm{lb}}(A)$. Suponhamos que $\mu_{\mathrm{lb}}(A)<+\infty$. Pelo item (a), existe $E \subset A \sigma$-finito para $\mu$ tal que $\mu(E)=\mu_{\mathrm{lb}}(A)$. Se $E=A$ então $\mu(A)=\mu(E)=\mu_{\mathrm{lb}}(A)$. Se $E \neq A$, para cada $E^{\prime} \subset A \backslash E$ subconjunto mensurável de medida finita, temos $\mu\left(E \cup E^{\prime}\right)<+\infty$, uma vez que $E$ também tem medida finita. Assim,

$$
\mu(E) \leq \mu(E)+\mu\left(E^{\prime}\right)=\mu\left(E \cup E^{\prime}\right) \leq \mu_{\mathrm{lb}}(A)=\mu(E),
$$

ou seja, $\mu\left(E^{\prime}\right)=0$. Logo, para cada subconjunto mensurável $E^{\prime} \subset A \backslash E$ temos $\mu\left(E^{\prime}\right)=0$ ou $\mu\left(E^{\prime}\right)=+\infty$. Como $A \backslash E$ não é um bloco infinito, 
$A \backslash E$ tem medida finita, e conseqüentemente nula. Desse modo, temos $\mu(A)=\mu(E)+\mu(A \backslash E)=\mu(E)=\mu_{\mathrm{lb}}(A)$.

- Prova de (c).

Vamos mostrar inicialmente que $\mu_{\mathrm{lb}}$ é uma medida. Observamos que se $B, B^{\prime} \in \mathcal{A}$ com $B \subset B^{\prime}$ então $\mu_{\mathrm{lb}}(B) \leq \mu_{\mathrm{lb}}\left(B^{\prime}\right)$. Com efeito, se $B \subset B^{\prime}$ então:

$$
\begin{aligned}
\mu_{\mathrm{lb}}(B) & =\sup \{\mu(E): E \subset B, E \in \mathcal{A} \text { e } \mu(E)<+\infty\} \\
& \leq \sup \left\{\mu(E): E \subset B^{\prime}, E \in \mathcal{A} \text { e } \mu(E)<+\infty\right\}=\mu_{\mathrm{lb}}\left(B^{\prime}\right) .
\end{aligned}
$$

É fácil ver que $\mu_{\mathrm{lb}}(\emptyset)=0$, pois

$$
\{\mu(E): E \subset \emptyset, E \in \mathcal{A} \text { e } \mu(E)<+\infty\}=\{0\} .
$$

Seja $\left(A_{n}\right)_{n \geq 1}$ uma seqüência de conjuntos mensuráveis, dois a dois disjuntos. Então $A=\bigcup_{n \geq 1} A_{n}$ é mensurável e existe, pelo item (a), $E \subset A$ $\sigma$-finito para $\mu$ com $\mu(E)=\mu_{\mathrm{lb}}(A)$. Ainda pelo item (a), para cada $n \geq 1$, existe $E_{n} \subset A_{n} \sigma$-finito para $\mu$ com $\mu\left(E_{n}\right)=\mu_{\mathrm{lb}}\left(A_{n}\right)$. Observe que sendo $E \sigma$-finito para $\mu, E \cap A_{n}$ é $\sigma$-finito para $\mu$ para cada $n \geq 1$; e como $E_{n}$ é $\sigma$-finito para $\mu$ para todo $n \geq 1$, temos que $\bigcup_{n \geq 1} E_{n}$ é $\sigma$-finito para $\mu$. Logo, pelo item (b), $\mu\left(\bigcup_{n \geq 1} E_{n}\right)=\mu_{\mathrm{lb}}\left(\bigcup_{n \geq 1} E_{n}\right)$ e $\mu\left(E \cap A_{n}\right)=\mu_{\mathrm{lb}}\left(E \cap A_{n}\right)$ para cada $n \geq 1$. Assim,

$$
\begin{aligned}
& \sum_{n \geq 1} \mu_{\mathrm{lb}}\left(A_{n}\right)=\sum_{n \geq 1} \mu\left(E_{n}\right)=\mu\left(\bigcup_{n \geq 1} E_{n}\right)=\mu_{\mathrm{lb}}\left(\bigcup_{n \geq 1} E_{n}\right) \leq \mu_{\mathrm{lb}}(A)=\mu(E) \\
& =\mu\left(\bigcup_{n \geq 1}\left(E \cap A_{n}\right)\right)=\sum_{n \geq 1} \mu\left(E \cap A_{n}\right)=\sum_{n \geq 1} \mu_{\mathrm{lb}}\left(E \cap A_{n}\right) \leq \sum_{n \geq 1} \mu_{\mathrm{lb}}\left(A_{n}\right) .
\end{aligned}
$$

Portanto $\mu_{\mathrm{lb}}(A)=\mu_{\mathrm{lb}}\left(\bigcup_{n \geq 1} A_{n}\right)=\sum_{n \geq 1} \mu_{\mathrm{lb}}\left(A_{n}\right)$. Vejamos agora que $\mu_{\mathrm{lb}}$ é livre de blocos. Se $A \in \mathcal{A}$ e $\mu_{\mathrm{lb}}(A)=+\infty$ então existe um subconjunto mensurável $E \subset A$ com $0<\mu(E)<+\infty$. De fato, se para todo $E \subset A, E \in \mathcal{A}$, com $\mu(E)<+\infty$ tivéssemos $\mu(E)=0$ então teríamos $\mu_{\mathrm{lb}}(A)=0$. Como $\mu(E)<+\infty, E$ é $\sigma$-finito para $\mu$, e portanto $\mu_{\mathrm{lb}}(E)=\mu(E)$. Logo $E$ é um subconjunto mensurável de $A$ com $0<\mu_{\mathrm{lb}}(E)<+\infty$, e isso mostra que $A$ não é um bloco infinito para $\mu_{\mathrm{lb}}$.

- Prova de (d). 
Se $A \in \mathcal{A}$ é $\sigma$-finito para $\mu_{\mathrm{lb}}$, então existe uma seqüência $\left(A_{n}\right)_{n \geq 1}$ de conjuntos mensuráveis, com $\mu_{\mathrm{lb}}\left(A_{n}\right)<+\infty$ para todo $n \geq 1$, tal que $A=\bigcup_{n \geq 1} A_{n}$. Pelo item (a), para cada $n \geq 1$ existe $E_{n} \subset A_{n} \sigma$-finito para $\mu \operatorname{com} \mu\left(E_{n}\right)=\mu_{\mathrm{lb}}\left(A_{n}\right)$, e pelo item (b) temos $\mu_{\mathrm{lb}}\left(E_{n}\right)=\mu\left(E_{n}\right)$ para todo $n \geq 1$. Logo $\mu_{\mathrm{lb}}\left(E_{n}\right)=\mu_{\mathrm{lb}}\left(A_{n}\right)$, e portanto $\mu_{\mathrm{lb}}\left(A_{n} \backslash E_{n}\right)=0$, para todo $n \geq 1$, uma vez que $\mu_{\mathrm{lb}}\left(A_{n}\right)=\mu_{\mathrm{lb}}\left(E_{n}\right)+\mu_{\mathrm{lb}}\left(A_{n} \backslash E_{n}\right)$ e $\mu_{\mathrm{lb}}\left(A_{n}\right)<+\infty$, para cada $n \geq 1$. Sejam

$$
A_{0}=\bigcup_{n \geq 1} E_{n} \quad \text { e } \quad A_{\infty}=A \backslash A_{0} .
$$

Como $A \in \mathcal{A}$ e $E_{n} \in \mathcal{A}$ para todo $n \geq 1$, segue que $A_{0}, A_{\infty} \in \mathcal{A}$. Além disso, $A=A_{0} \cup A_{\infty}$ e $A_{0} \cap A_{\infty}=\emptyset$, pois $A_{\infty}=A \backslash A_{0}$. Ainda, para todo $n \geq 1, E_{n}$ é mensurável e $\mu\left(E_{n}\right)=\mu_{\mathrm{lb}}\left(A_{n}\right)<+\infty, \log \mathrm{O} A_{0}$ é $\sigma$-finito para $\mu$. Por outro lado, se $x \in A_{\infty}$ então $x \in A$ e $x \notin A_{0}$, ou seja, $x \in A_{n}$ para algum $n \geq 1$. Portanto existe $n \geq 1$ tal que $x \in A_{n} \backslash E_{n} \subset \bigcup_{n \geq 1}\left(A_{n} \backslash E_{n}\right)$. Assim,

$$
\mu_{\mathrm{lb}}\left(A_{\infty}\right) \leq \mu_{\mathrm{lb}}\left(\bigcup_{n \geq 1}\left(A_{n} \backslash E_{n}\right)\right) \leq \sum_{n \geq 1} \mu_{\mathrm{lb}}\left(A_{n} \backslash E_{n}\right)=0,
$$

i.e., $\mu_{\mathrm{lb}}\left(A_{\infty}\right)=0$.

DefiniçÃo 2.1.8. Seja $(X, \mathcal{A}, \mu)$ é um espaço de medida. A medida $\mu_{\mathrm{lb}}: \mathcal{A} \rightarrow[0,+\infty]$, definida como em (2.1.1), é chamada de versão livre de blocos da medida $\mu$.

Observamos que, pelo item (b) da Proposição 2.1.7, se $\mu$ é uma medida livre de blocos então ela é igual à sua versão livre de blocos, ou seja, $\mu_{\mathrm{lb}}=\mu$ se $\mu$ é livre de blocos.

Vimos anteriormente que se $(X, \mathcal{A}, \mu)$ é um espaço de medida então $\mathcal{M}(X, \mathcal{A})$ denota o espaço vetorial real das funções mensuráveis a valores reais sobre $X, \mathcal{M}_{o}(X, \mathcal{A}, \mu)$ denota o subespaço de $\mathcal{M}(X, \mathcal{A})$ das funções nulas $\mu$-qs, e $\overline{\mathcal{M}}(X, \mathcal{A}, \mu)$ denota o quociente de $\mathcal{M}(X, \mathcal{A})$ por $\mathcal{M}_{o}(X, \mathcal{A}, \mu)$. Se $f \in \mathcal{M}_{o}(X, \mathcal{A}, \mu)$ então $f=0 \mu$-qs, i.e., existe $A \in \mathcal{A} \operatorname{com} \mu(A)=0$ tal que $f(x)=0$ para todo $x \in X \backslash A$. Uma vez que $\mu(A)=0$ implica $\mu_{\mathrm{lb}}(A)=0$, segue que $f=0 \mu_{\mathrm{lb}}$-qs, e portanto $f \in \mathcal{M}_{o}\left(X, \mathcal{A}, \mu_{\mathrm{lb}}\right)$. Isso significa que $\mathcal{M}_{o}(X, \mathcal{A}, \mu)$ é subconjunto de $\mathcal{M}_{o}\left(X, \mathcal{A}, \mu_{\mathrm{lb}}\right)$, e assim, pela Proposição 1.2.2, a aplicação identidade $\operatorname{Id}: \mathcal{M}(X, \mathcal{A}) \rightarrow \mathcal{M}(X, \mathcal{A})$ induz a 
aplicação linear

$$
\overline{\mathcal{M}}(X, \mathcal{A}, \mu) \ni[f]_{\mu} \longmapsto[f]_{\mu_{\mathrm{lb}}} \in \overline{\mathcal{M}}\left(X, \mathcal{A}, \mu_{\mathrm{lb}}\right) .
$$

Além disso, como $\mu_{\mathrm{lb}} \leq \mu$, tal aplicação leva $L^{p}(X, \mathcal{A}, \mu)$ em $L^{p}\left(X, \mathcal{A}, \mu_{\mathrm{lb}}\right)$, para todo $p \in[1,+\infty]$, e portanto é também linear a aplicação

$$
L^{p}(X, \mathcal{A}, \mu) \ni[f]_{\mu} \longmapsto[f]_{\mu_{\mathrm{lb}}} \in L^{p}\left(X, \mathcal{A}, \mu_{\mathrm{lb}}\right) .
$$

Proposição 2.1.9. Seja $(X, \mathcal{A}, \mu)$ um espaço de medida. Para todo $p \in[1,+\infty[$, a aplicação (2.1.2) é uma isometria linear.

Demonstração. Chamemos de $\phi$ a aplicação (2.1.2). Das considerações anteriores segue imediatamente que $\phi$ é linear. Vamos mostrar primeiramente que $\phi$ está bem definida e é uma imersão isométrica e em seguida mostraremos a sobrejetividade de $\phi$. Se $p \in\left[1,+\infty\left[\right.\right.$ e $f \in L^{p}(X, \mathcal{A}, \mu)$ então, pelo Lema 2.1.4, o conjunto $A=f^{-1}(\mathbb{R} \backslash\{0\})$ é $\sigma$-finito para $\mu$. Portanto, pelo item (b) da Proposição 2.1.7 temos que $\mu(E)=\mu_{\mathrm{lb}}(E)$ para todo subconjunto mensurável $E \subset A$. Assim,

$$
\int_{X}|f|^{p} \mathrm{~d} \mu_{\mathrm{lb}}=\int_{A}|f|^{p} \mathrm{~d} \mu_{\mathrm{lb}}=\int_{A}|f|^{p} \mathrm{~d} \mu=\int_{X}|f|^{p} \mathrm{~d} \mu<+\infty,
$$

i.e., $f \in L^{p}\left(X, \mathcal{A}, \mu_{\mathrm{lb}}\right)$, e portanto $\phi$ está bem definida. Além disso,

$$
\left\|[f]_{\mu}\right\|_{p}=\left(\int_{X}|f|^{p} \mathrm{~d} \mu\right)^{\frac{1}{p}}=\left(\int_{X}|f|^{p} \mathrm{~d} \mu_{\mathrm{lb}}\right)^{\frac{1}{p}}=\left\|[f]_{\mu_{\mathrm{lb}}}\right\|_{p},
$$

ou seja, $\phi$ é uma imersão isométrica. Para mostrar que a aplicação $\phi$ é sobrejetora vamos fixar $f \in L^{p}\left(X, \mathcal{A}, \mu_{\mathrm{lb}}\right)$ e exibir $g \in L^{p}(X, \mathcal{A}, \mu)$ tal que $g=f$ $\mu_{\mathrm{lb} \text {-qs. }}$ Daí seguirá que $\phi\left([g]_{\mu}\right)=[g]_{\mu_{\mathrm{lb}}}=[f]_{\mu_{\mathrm{lb}}}$. Se $f \in L^{p}\left(X, \mathcal{A}, \mu_{\mathrm{lb}}\right)$ então $A=f^{-1}(\mathbb{R} \backslash\{0\})$ é $\sigma$-finito para $\mu_{\mathrm{lb}}$. Logo, pelo item (d) da Proposição 2.1.7, existem $A_{0}, A_{\infty} \in \mathcal{A}$ tais que $A=A_{0} \cup A_{\infty}, A_{0} \cap A_{\infty}=\emptyset$, $A_{0}$ é $\sigma$-finito para $\mu$ e $\mu_{\mathrm{lb}}\left(A_{\infty}\right)=0$. A função $g=f \chi_{A_{0}}$ é claramente mensurável, e uma vez que $\mu_{\mathrm{lb}}\left(A_{\infty}\right)=0$ e

$$
g(x)= \begin{cases}f(x) & \text { se } x \in A_{0} \\ 0 \neq f(x) & \text { se } x \in A_{\infty} \\ 0=f(x) & \text { se } x \in X \backslash A\end{cases}
$$

temos $g=f \mu_{\mathrm{lb}}$-qs. Por outro lado, sendo $A_{0} \sigma$-finito para $\mu$, pelo item (b) da Proposição 2.1.7 temos que $\mu$ e $\mu_{\mathrm{lb}}$ coincidem em todos os subconjuntos 
mensuráveis de $A_{0}$. Assim,

$$
\begin{aligned}
\int_{X}|g|^{p} \mathrm{~d} \mu=\int_{X}\left|f \chi_{A_{0}}\right|^{p} \mathrm{~d} \mu=\int_{X}|f|^{p} \chi_{A_{0}} \mathrm{~d} \mu=\int_{A_{0}}|f|^{p} \mathrm{~d} \mu \\
=\int_{A_{0}}|f|^{p} \mathrm{~d} \mu_{\mathrm{lb}}=\int_{X}|f|^{p} \mathrm{~d} \mu_{\mathrm{lb}}<+\infty,
\end{aligned}
$$

sendo a última igualdade verdadeira porque $\mu_{\mathrm{lb}}\left(A_{\infty}\right)=0$ e $\left.f\right|_{A^{c}}=0$. Logo, $g \in L^{p}(X, \mathcal{A}, \mu)$, o que mostra a sobrejetividade de $\phi$, e portanto a aplicação (2.1.2) é, de fato, uma isometria linear para todo $p \in[1,+\infty[$.

Proposição 2.1.10. Seja $(X, \mathcal{A}, \mu)$ um espaço de medida. Para $p=+\infty$, (2.1.2) é uma aplicação quociente ${ }^{1}$, e os elementos não nulos de seu núcleo são as funções $f \in L^{\infty}(X, \mathcal{A}, \mu)$ tais que $f^{-1}(\mathbb{R} \backslash\{0\})$ é um bloco infinito para $\mu$.

Demonstração. Seja $\phi$ a aplicação (2.1.2) para $p=+\infty$. Queremos mostrar que $\phi$ é uma aplicação quociente, ou seja, que $\phi$ é uma aplicação linear limitada sobrejetora tal que, para toda $f \in L^{\infty}(X, \mathcal{A}, \mu)$,

$$
\left\|[f]_{\mu_{\mathrm{lb}}}\right\|_{\infty}=\inf \left\{\left\|[g]_{\mu}\right\|_{\infty}: g \in L^{\infty}(X, \mathcal{A}, \mu), g=f \mu_{\mathrm{lb}-\mathrm{qs}}\right\} .
$$

Mostraremos inicialmente que $\phi$ é sobrejetora. Sejam $f \in L^{\infty}\left(X, \mathcal{A}, \mu_{\mathrm{lb}}\right)$ fixada e $\alpha=\left\|[f]_{\mu_{\mathrm{lb}}}\right\|_{\infty}$. Afirmamos que a função $f_{0}: X \rightarrow \mathbb{R}$, dada por

$$
f_{0}(x)= \begin{cases}f(x) & \text { se }|f(x)| \leq \alpha \\ 0 & \text { caso contrário }\end{cases}
$$

está em $L^{\infty}(X, \mathcal{A}, \mu)$ e $f_{0}=f \mu_{\mathrm{lb}}$-qs. De fato, como $f$ é uma função mensurável, o conjunto $A \stackrel{\text { def }}{=}\{x \in X:|f(x)| \leq \alpha\}$ é mensurável, e uma vez que $f_{0}=f \chi_{A}$ segue que $f_{0}$ é mensurável. Além disso, sendo $f_{0}$ limitada, é claro que $f_{0}$ é $\mu$-qs limitada, e portanto $f_{0} \in L^{\infty}(X, \mathcal{A}, \mu)$. Sendo $\alpha=\left\|[f]_{\mu_{\mathrm{lb}}}\right\|_{\infty}$, temos pela Observação 1.4 .77 que $|f| \leq \alpha \mu_{\mathrm{lb}}$-qs, isto é, existe $B \in \mathcal{A}$ com $\mu_{\mathrm{lb}}(B)=0$ tal que $|f(x)| \leq \alpha$ para todo $x \in B^{c}$. Portanto $f_{0}(x)=f(x)$ para todo $x \in B^{c}$, ou seja, $f_{0}=f \mu_{\mathrm{lb}}$-qs. Desse modo, $\phi\left(\left[f_{0}\right]_{\mu}\right)=\left[f_{0}\right]_{\mu_{\mathrm{lb}}}=[f]_{\mu_{\mathrm{lb}}}$, o que prova que $\phi$ é sobrejetora. Seja $f \in L^{\infty}(X, \mathcal{A}, \mu)$ arbitrária, e sejam $\alpha$ e $f_{0}$ como acima. Vamos mostrar agora que $\left\|[g]_{\mu}\right\|_{\infty} \geq \alpha$ para cada $g \in L^{\infty}(X, \mathcal{A}, \mu)$ com $g=f \mu_{\mathrm{lb}}$-qs, e que a norma de $f_{0}$ em $L^{\infty}(X, \mathcal{A}, \mu)$ é igual a $\alpha$. Isso implicará em (2.1.3).

\footnotetext{
${ }^{1}$ No sentido da definição 1.3.8.
} 
Seja $g \in L^{\infty}(X, \mathcal{A}, \mu)$ com $g=f \mu_{\mathrm{lb}}$-qs. Sabemos que $\left\|[f]_{\mu_{\mathrm{lb}}}\right\|_{\infty}=\alpha$, então $\mu_{\mathrm{lb}}(\{x \in X:|f(x)|>\alpha-\varepsilon\})>0$ para cada $\varepsilon>0$ (senão teríamos $|f| \leq \alpha-\varepsilon \mu_{\mathrm{lb}}$-qs), e portanto $\alpha=\inf \left\{c \geq 0:|f| \leq c \mu_{\mathrm{lb}}\right.$-qs $\} \leq \alpha-\varepsilon$. Assim, visto que $g=f \mu_{\mathrm{lb}}$-qs, para cada $\varepsilon>0$ temos:

$$
\begin{aligned}
\mu(\{x \in X:|g(x)|>\alpha-\varepsilon\}) & \geq \mu_{\mathrm{lb}}(\{x \in X:|g(x)|>\alpha-\varepsilon\}) \\
& =\mu_{\mathrm{lb}}(\{x \in X:|f(x)|>\alpha-\varepsilon\})>0 .
\end{aligned}
$$

Logo, para todo $\varepsilon>0, \alpha-\varepsilon$ não pertence ao conjunto

$$
A \stackrel{\text { def }}{=}\{c \geq 0:|g| \leq c \mu \text {-qs }\} .
$$

Como $\left\|[g]_{\mu}\right\|_{\infty}=\min A$, segue que $\left\|[g]_{\mu}\right\|_{\infty} \geq \alpha$; em particular temos $\left\|\left[f_{0}\right]_{\mu}\right\|_{\infty} \geq \alpha$, pois $f_{0} \in L^{\infty}(X, \mathcal{A}, \mu)$ e $f_{0}=f \mu_{\mathrm{lb}}$-qs. Por outro lado, $\left|f_{0}(x)\right| \leq \alpha$ para todo $x \in X$, o que implica $\left\|\left[f_{0}\right]_{\mu}\right\|_{\infty} \leq \alpha$. Seja

$$
B=\left\{\left\|[g]_{\mu}\right\|_{\infty}: g \in L^{\infty}(X, \mathcal{A}, \mu), g=f \mu_{\mathrm{lb}-\mathrm{qs}}\right\} .
$$

Sabemos que $f_{0} \in L^{\infty}(X, \mathcal{A}, \mu), f_{0}=f \mu_{\mathrm{lb}}$-qs e $\alpha=\left\|\left[f_{0}\right]_{\mu}\right\|_{\infty} ; \log \alpha \in B$, e portanto inf $B \leq \alpha$. Vimos que $\left\|[g]_{\mu}\right\|_{\infty} \geq \alpha$ para toda $g \in L^{\infty}(X, \mathcal{A}, \mu)$ com $g=f \mu_{\mathrm{lb}}$-qs, ou seja, $\alpha$ é uma cota inferior de $B$, e assim $\alpha \leq \inf B$. Portanto $\left\|[f]_{\mu_{\mathrm{lb}}}\right\|_{\infty}=\alpha=\inf B$. Para cada $f \in L^{\infty}(X, \mathcal{A}, \mu)$ temos que

$$
\left\|\phi\left([f]_{\mu}\right)\right\|_{\infty}=\left\|[f]_{\mu_{\mathrm{lb}}}\right\|_{\infty} \leq\left\|[f]_{\mu}\right\|_{\infty},
$$

e isso mostra que $\phi$ é uma aplicação linear limitada. Por fim, se $f \in \operatorname{Ker}(\phi)$ então $\phi\left([f]_{\mu}\right)=[f]_{\mu_{\mathrm{lb}}}=[0]_{\mu_{\mathrm{lb}}}$, ou seja, $f=0 \mu_{\mathrm{lb}}$-qs, e portanto

$$
\mu_{\mathrm{lb}}\left(f^{-1}(\mathbb{R} \backslash\{0\})\right)=0 .
$$

Logo, $\mu\left(f^{-1}(\mathbb{R} \backslash\{0\})\right)=0$ ou $f^{-1}(\mathbb{R} \backslash\{0\})$ é um bloco infinito. Observe que se $\mu\left(f^{-1}(\mathbb{R} \backslash\{0\})\right)=0$ então $f=0 \mu$-qs. Portanto os elementos não nulos do núcleo da aplicação $\phi$ são as funções $f \in L^{\infty}(X, \mathcal{A}, \mu)$ tais que $f^{-1}(\mathbb{R} \backslash\{0\})$ é um bloco infinito para $\mu$.

Seja $(X, \mathcal{A}, \mu)$ um espaço de medida. Para $p, q \in[1,+\infty] \operatorname{com} \frac{1}{p}+\frac{1}{q}=1$ é comutativo o diagrama

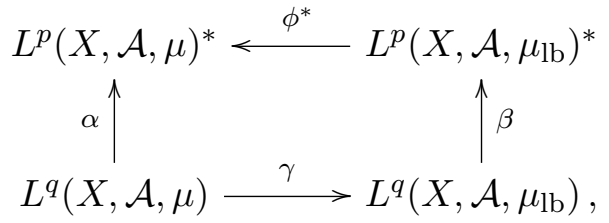


no qual $\alpha$ e $\beta$ são $(q, p)$-aplicações de Riesz (1.4.2) para os espaços $(X, \mathcal{A}, \mu)$ e $\left(X, \mathcal{A}, \mu_{\mathrm{lb}}\right), \phi^{*}$ é a transposta da aplicação (2.1.2), $\gamma$ é a versão da aplicação (2.1.2) para $L^{q}$. Com efeito, se $[g]_{\mu} \in L^{q}(X, \mathcal{A}, \mu)$ então

$$
\gamma\left([g]_{\mu}\right)=[g]_{\mu_{\mathrm{lb}}} \in L^{q}\left(X, \mathcal{A}, \mu_{\mathrm{lb}}\right) .
$$

Aplicando $\beta$ obtemos $\beta_{g} \in L^{p}\left(X, \mathcal{A}, \mu_{\mathrm{lb}}\right)^{*}$ tal que, para cada $h \in L^{p}\left(X, \mathcal{A}, \mu_{\mathrm{lb}}\right)$, $\beta_{g}(h)=\int_{X} h g \mathrm{~d} \mu_{\mathrm{lb}}$. Portanto, para toda $f \in L^{p}(X, \mathcal{A}, \mu)$, temos:

$$
\phi^{*}\left(\beta_{g}\right)\left([f]_{\mu}\right)=\left(\beta_{g} \circ \phi\right)\left([f]_{\mu}\right)=\beta_{g}\left([f]_{\mu_{\mathrm{lb}}}\right)=\int_{X} f g \mathrm{~d} \mu_{\mathrm{lb}} .
$$

Por outro lado, $\alpha(g)=\alpha_{g} \in L^{p}(X, \mathcal{A}, \mu)^{*}$ tal que, para cada $f \in L^{p}(X, \mathcal{A}, \mu)$, $\alpha_{g}\left([f]_{\mu}\right)=\int_{X} f g \mathrm{~d} \mu$. Sendo $f \in L^{p}(X, \mathcal{A}, \mu)$ temos, pelo Lema 2.1.4, $A=f^{-1}(\mathbb{R} \backslash\{0\}) \sigma$-finito para $\mu$, e portanto $\mu(A)=\mu_{\mathrm{lb}}(A)$, pelo item (b) da Proposição 2.1.7. Assim, como $f g$ se anula onde $f$ se anula, segue que

$$
\int_{X} f g \mathrm{~d} \mu=\int_{A} f g \mathrm{~d} \mu=\int_{A} f g \mathrm{~d} \mu_{\mathrm{lb}}=\int_{X} f g \mathrm{~d} \mu_{\mathrm{lb}},
$$

o que mostra que o diagrama (2.1.4) comuta. Se $p, q \in] 1,+\infty[$ então (2.1.4) é apenas um diagrama comutativo de isometrias. O caso mais interessante ocorre para $p=1$ e $q=+\infty$; nesse caso temos o seguinte diagrama comutativo:

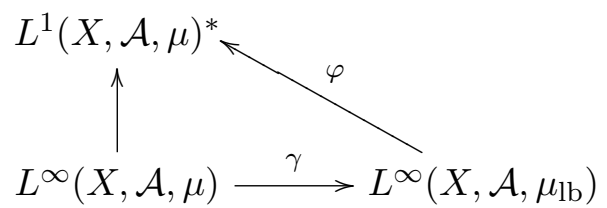

Nele, $\varphi$ é uma aplicação que difere da aplicação de $\operatorname{Riesz}$ do espaço $\left(X, \mathcal{A}, \mu_{\mathrm{lb}}\right)$ por uma isometria. De fato, é comutativo o diagrama:

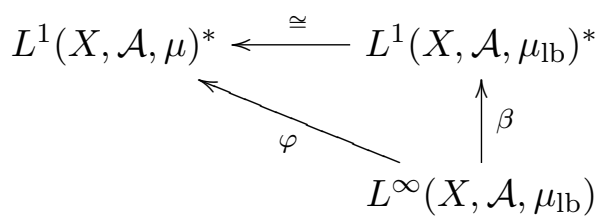

Para $p=1$ a aplicação (2.1.2) é uma isometria linear, e portanto a sua transposta também. Uma vez que $\mu_{\mathrm{lb}}$ é uma medida livre de blocos, pela Proposição 2.1.5, $\beta$ é uma imersão isométrica. Logo, $\varphi$ é uma imersão isométrica, pois é a composição de duas imersões isométricas. Portanto, o 
diagrama (2.1.5) mostra-nos como decompor a aplicação de Riesz do espaço $(X, \mathcal{A}, \mu)$ em uma imersão isométrica $(\varphi)$ e uma aplicação quociente $(\gamma)$.

\subsection{Medidas cheias}

Proposição 2.2.1. Se $(X, \overline{\mathcal{A}}, \bar{\mu})$ é o completamento do espaço de medida $(X, \mathcal{A}, \mu)$ então, para cada $p \in[1,+\infty]$ a aplicação inclusão de $\mathcal{M}(X, \mathcal{A})$ em $\mathcal{M}(X, \overline{\mathcal{A}})$ induz a isometria linear:

$$
L^{p}(X, \mathcal{A}, \mu) \ni[f]_{\mu} \longmapsto[f]_{\bar{\mu}} \in L^{p}(X, \overline{\mathcal{A}}, \bar{\mu}) .
$$

Demonstração. Seja $\phi$ a aplicação (2.2.1). Pela Proposição 1.2.2, $\phi$ é linear. Para mostrar que $\phi$ preserva norma vamos considerar os casos $p \in[1,+\infty[$ e $p=+\infty$. Suponhamos primeiramente que $p \in[1,+\infty[$. Uma vez que $\mathcal{A} \subset \overline{\mathcal{A}}$ e $\left.\bar{\mu}\right|_{\mathcal{A}}=\mu$, pelo Corolário 1.4 .67 temos

$$
\left\|[f]_{\mu}\right\|_{p}=\left(\int_{X}|f|^{p} \mathrm{~d} \mu\right)^{\frac{1}{p}}=\left(\int_{X}|f|^{p} \mathrm{~d} \bar{\mu}\right)^{\frac{1}{p}}=\left\|[f]_{\bar{\mu}}\right\|_{p},
$$

para cada $f \in L^{p}(X, \mathcal{A}, \mu)$. Consideremos agora o caso $p=+\infty$. Se $f$ está em $L^{\infty}(X, \mathcal{A}, \mu)$ então $B \stackrel{\text { def }}{=}\{x \in X:|f(x)|>c\} \in \mathcal{A}$ para todo $c \in \mathbb{R}$. Logo, $\mu(B)=0$ se e somente se $\bar{\mu}(B)=0$, e portanto $|f| \leq c \mu$-qs se e somente se $|f| \leq c \bar{\mu}$-qs. Assim,

$\left\|[f]_{\mu}\right\|_{\infty}=\inf \{c \geq 0:|f| \leq c \mu$-qs $\}=\inf \{c \geq 0:|f| \leq c \bar{\mu}$-qs $\}=\left\|[f]_{\bar{\mu}}\right\|_{\infty}$.

Portanto $\phi$ é uma imersão isométrica. Seja $f \in L^{p}(X, \overline{\mathcal{A}}, \bar{\mu})$ fixada. Como $f$ é mensurável em relação a $\overline{\mathcal{A}}$, pela Proposição 1.4 .69 existe $\widetilde{f}: X \rightarrow \mathbb{R}$ mensurável relativamente a $\mathcal{A}$ com $\widetilde{f}=f \bar{\mu}$-qs, e pelo que vimos acima, $\left\|[\tilde{f}]_{\mu}\right\|_{p}=\left\|[\widetilde{f}]_{\bar{\mu}}\right\|_{p}=\left\|[f]_{\bar{\mu}}\right\|_{p}<+\infty$. Portanto, $\widetilde{f}$ está em $L^{p}(X, \mathcal{A}, \mu)$ e $\phi\left([\tilde{f}]_{\mu}\right)=[\tilde{f}]_{\bar{\mu}}=[f]_{\bar{\mu}}$, o que mostra a sobrejetividade de $\phi$.

Seja $(X, \mathcal{A}, \mu)$ um espaço de medida. Para $p, q \in[1,+\infty] \operatorname{com} \frac{1}{p}+\frac{1}{q}=1$ é comutativo o diagrama:

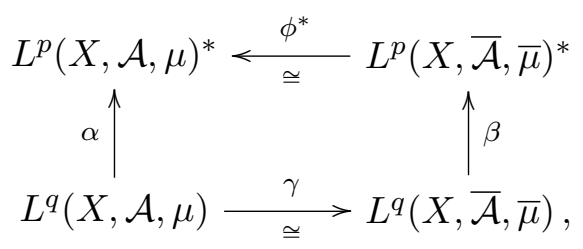


no qual $\alpha$ e $\beta$ são $(q, p)$-aplicações de Riesz (1.4.2) para os espaços $(X, \mathcal{A}, \mu)$ e $(X, \overline{\mathcal{A}}, \bar{\mu}), \phi^{*}$ é a transposta da aplicação $(2.2 .1)$ e $\gamma$ é a versão da aplicação (2.2.1) para $L^{q}$. Com efeito, se $[g]_{\mu} \in L^{q}(X, \mathcal{A}, \mu)$ então

$$
\gamma\left([g]_{\mu}\right)=[g]_{\bar{\mu}} \in L^{q}(X, \overline{\mathcal{A}}, \bar{\mu}) .
$$

Aplicando $\beta$ obtemos $\beta_{g} \in L^{p}(X, \overline{\mathcal{A}}, \bar{\mu})^{*}$ tal que, para cada $h \in L^{p}(X, \overline{\mathcal{A}}, \bar{\mu})$, $\beta_{g}(h)=\int_{X} h g \mathrm{~d} \bar{\mu}$. Assim, para toda $f \in L^{p}(X, \mathcal{A}, \mu)$, temos:

$$
\phi^{*}\left(\beta_{g}\right)\left([f]_{\mu}\right)=\left(\beta_{g} \circ \phi\right)\left([f]_{\mu}\right)=\beta_{g}\left([f]_{\bar{\mu}}\right)=\int_{X} f g \mathrm{~d} \bar{\mu} .
$$

Por outro lado, $\alpha(g)=\alpha_{g} \in L^{p}(X, \mathcal{A}, \mu)^{*}$ tal que, para cada $f \in L^{p}(X, \mathcal{A}, \mu)$, $\alpha_{g}\left([f]_{\mu}\right)=\int_{X} f g \mathrm{~d} \mu$. Portanto, uma vez que $\mathcal{A} \subset \overline{\mathcal{A}}$ e $\mu=\left.\bar{\mu}\right|_{\mathcal{A}}$, pelo Corolário 1.4.67 temos $\int_{X} f g \mathrm{~d} \mu=\int_{X} f g \mathrm{~d} \bar{\mu}$, o que mostra que o diagrama (2.2.2) comuta.

Nesta seção são estudados outros tipos de "completamento" de espaços de medida, os quais estão relacionados ao Teorema de Representação de Riesz. Veremos a seguir um exemplo em que a aplicação de Riesz (1.4.4) não é sobrejetora.

Exemplo 2.2.2. Sejam $X$ um conjunto não enumerável e $\mathcal{A}$ a $\sigma$-álgebra consistindo de todos os subconjuntos de $X$ que são enumeráveis ou têm complementar enumerável. Seja $\mu: \mathcal{A} \rightarrow[0,+\infty]$ a medida de contagem. Então $L^{1}(X, \mathcal{A}, \mu)$ é o espaço das funções $f: X \rightarrow \mathbb{R} \operatorname{com} \sum_{x \in X}|f(x)|<+\infty$. De fato, seja $f$ uma aplicação em $\mathcal{M}(X, \mathcal{A})$; se o conjunto $A \stackrel{\text { def }}{=} f^{-1}(\mathbb{R} \backslash\{0\})$ é enumerável então, pela Observação 1.4.70, temos:

$$
\int_{X}|f| \mathrm{d} \mu=\int_{A}|f| \mathrm{d} \mu=\int_{\bigcup_{x \in A}\{x\}}|f| \mathrm{d} \mu=\sum_{x \in A} \int_{\{x\}}|f| \mathrm{d} \mu .
$$

E assim, $\int_{X}|f| \mathrm{d} \mu=\sum_{x \in A} \int_{\{x\}}|f(x)| \mathrm{d} \mu=\sum_{x \in A} \int_{X}|f(x)| \chi_{\{x\}} \mathrm{d} \mu$

$$
=\sum_{x \in A}|f(x)| \mu(\{x\})=\sum_{x \in X}|f(x)| .
$$

Por outro lado, se $A$ é não enumerável então existe $\varepsilon>0$ tal que é infinito o conjunto $B \stackrel{\text { def }}{=}\{x \in X:|f(x)|>\varepsilon\} \subset A$. Então:

$$
\int_{X}|f| \mathrm{d} \mu=\int_{A}|f| \mathrm{d} \mu \geq \int_{B}|f| \mathrm{d} \mu>\int_{B} \varepsilon \mathrm{d} \mu>\varepsilon \mu(B)=+\infty \quad \text { e }
$$




$$
\sum_{x \in X}|f(x)|=\sum_{x \in A}|f(x)| \geq \sum_{x \in B}|f(x)|=+\infty
$$

i.e., $\int_{X}|f| \mathrm{d} \mu=+\infty=\sum_{x \in X}|f(x)|$. Portanto, se $f \in L^{1}(X, \mathcal{A}, \mu)$ então $\sum_{x \in X}|f(x)|<+\infty$, e conseqüentemente o conjunto $A=f^{-1}(\mathbb{R} \backslash\{0\})$ é enumerável. Por outro lado, se $f: X \rightarrow \mathbb{R}$ é tal que $\sum_{x \in X}|f(x)|<+\infty$ então $A$ é enumerável, e portanto $\left.f\right|_{A}$ é mensurável; como $\left.f\right|_{A^{c}}=0$ segue que $f$ é $\mathcal{A}$-mensurável, e uma vez que $\int_{X}|f| \mathrm{d} \mu=\sum_{x \in X}|f(x)|$ temos que $f$ está em $L^{1}(X, \mathcal{A}, \mu)$. Seja $S$ um subconjunto não enumerável de $X$ com $X \backslash S$ também não enumerável. Seja $\alpha: L^{1}(X, \mathcal{A}, \mu) \rightarrow \mathbb{R}$ a aplicação dada por $\alpha(f)=\sum_{x \in S} f(x)$. Afirmamos que $\alpha$ é um funcional linear limitado com $\|\alpha\|=1$ que não está na imagem da aplicação de Riesz (1.4.4). Com efeito, $\alpha$ é claramente linear, e para cada $f \in L^{1}(X, \mathcal{A}, \mu)$ temos:

$$
|\alpha(f)|=\left|\sum_{x \in S} f(x)\right| \leq \sum_{x \in S}|f(x)| \leq \sum_{x \in X}|f(x)|=\int_{X}|f| \mathrm{d} \mu=\|f\|_{1} .
$$

Logo $\alpha$ é limitado e $\|\alpha\| \leq 1$, uma vez que

$$
\|\alpha\|=\inf \left\{c \geq 0:|\alpha(f)| \leq c\|f\|_{1} \text {, para todo } f \in L^{1}(X, \mathcal{A}, \mu)\right\} .
$$

Por outro lado, sendo $S$ não enumerável, existe $x_{o} \in S$. Se $f=\chi_{\left\{x_{o}\right\}}$ então

$$
\|f\|_{1}=\int_{X}|f| \mathrm{d} \mu=\int_{X} \chi_{\left\{x_{o}\right\}} \mathrm{d} \mu=\mu\left(\left\{x_{o}\right\}\right)=1,
$$

e portanto $f \in L^{1}(X, \mathcal{A}, \mu)$. Além disso,

$$
\alpha(f)=\sum_{x \in S} f(x)=f\left(x_{o}\right)+\sum_{x \in S \backslash\left\{x_{o}\right\}} f(x)=1+0=1,
$$

o que implica $\|\alpha\|=\sup \left\{|\alpha(f)|: f \in L^{1}(X, \mathcal{A}, \mu),\|f\|_{1}=1\right\} \geq 1$. Suponhamos que $\alpha$ esteja na imagem da aplicação de Riesz (1.4.4), ou seja, que exista $g \in L^{\infty}(X, \mathcal{A}, \mu)$ tal que $\alpha_{g}=\alpha$. Então, para cada $x \in X$, temos $\chi_{\{x\}} \in L^{1}(X, \mathcal{A}, \mu) \mathrm{e}$

$$
\begin{aligned}
g(x)=g(x) \mu(\{x\})=\int_{\{x\}} g(x) \mathrm{d} \mu & =\int_{\{x\}} g \mathrm{~d} \mu=\int_{X} g \chi_{\{x\}} \mathrm{d} \mu \\
=\alpha_{g}\left(\chi_{\{x\}}\right)=\alpha\left(\chi_{\{x\}}\right) & =\sum_{y \in S} \chi_{\{x\}}(y)=\sum_{y \in S} \chi_{\{y\}}(x)=\chi_{S}(x) .
\end{aligned}
$$

Portanto, $g=\chi_{S}$. Mas $g$ é mensurável e $\chi_{S}$ não; logo $\alpha$ não está na imagem da aplicação de Riesz (1.4.4) para o espaço $(X, \mathcal{A}, \mu)$. 
Observamos que a não sobrejetividade da aplicação de Riesz no Exemplo 2.2.2 é causada pela escolha "ruim" da $\sigma$-álgebra para o domínio da medida $\mu$. Realmente, note que a aplicação $g=\chi_{S}$ tem a propriedade $\alpha(f)=\int_{X} f g \mathrm{~d} \mu$ para toda $f \in L^{1}(X, \mathcal{A}, \mu)$, mas $g$ não é um representante válido para o funcional $\alpha$ porque ela não é mensurável. Observamos contudo que a medida de contagem $\mu$ pode ser naturalmente estendida para a $\sigma$-álgebra $\mathcal{P}(X)$ de todos os subconjuntos de $X$. Tal extensão de $\mu$ não muda o espaço $L^{1}$ mas ela amplia o espaço $L^{\infty}$ de maneira tal que a aplicação de Riesz (1.4.4) seja uma isometria linear.

Definição 2.2.3. Seja $(X, \mathcal{A})$ um espaço mensurável. Uma medida $\mu: \mathcal{A} \rightarrow[0,+\infty]$ é chamada cheia se vale a seguinte propriedade: dado $A \subset X$ tal que $A \cap E \in \mathcal{A}$ para todo $E \in \mathcal{A}$ com $\mu(E)<+\infty$ tem-se $A \in \mathcal{A}$.

Dado um espaço de medida $(X, \mathcal{A}, \mu)$, designaremos por $\mathcal{A}_{\mathrm{c}}$ o conjunto:

$$
\mathcal{A}_{\mathrm{c}}=\{A \subset X: A \cap E \in \mathcal{A} \text {, para cada } E \in \mathcal{A} \operatorname{com} \mu(E)<+\infty\} .
$$

Afirmamos que $\mathcal{A}_{\mathrm{c}}$ é uma $\sigma$-álgebra de partes de $X$ que contém $\mathcal{A}$. De fato, para cada $A \in \mathcal{A}, A \cap E \in \mathcal{A}$ para todo $E \in \mathcal{A} \operatorname{com} \mu(E)<+\infty ; \log 0 \mathcal{A} \subset \mathcal{A}_{\mathrm{c}}$ e conseqüentemente $\mathcal{A}_{\mathrm{c}} \neq \emptyset$. Sejam $A \in \mathcal{A}_{\mathrm{c}}$ e $\left(A_{n}\right)_{n \geq 1}$ uma seqüência de elementos de $\mathcal{A}_{\mathrm{c}}$. Seja $E \in \mathcal{A} \operatorname{com} \mu(E)<+\infty$ arbitrário. Então $A^{c} \cap E=E \backslash A=E \backslash(A \cap E) \in \mathcal{A}$, e portanto $A^{c} \in \mathcal{A}_{\mathrm{c}}$. Ainda, uma vez que $A_{n} \in \mathcal{A}_{\mathrm{c}}$ para todo $n \geq 1$, temos que $\left(\bigcup_{n \geq 1} A_{n}\right) \cap E=\bigcup_{n \geq 1}\left(A_{n} \cap E\right) \in \mathcal{A}$, e assim $\bigcup_{n \geq 1} A_{n} \in \mathcal{A}_{\mathrm{c}}$. Seja $\mu_{\mathrm{c}}: \mathcal{A}_{\mathrm{c}} \rightarrow[0,+\infty]$ a aplicação definida por:

$$
\mu_{\mathrm{c}}(A)= \begin{cases}\mu(A) & \text { se } A \in \mathcal{A} \\ +\infty & \text { se } A \in \mathcal{A}_{\mathrm{c}} \backslash \mathcal{A}\end{cases}
$$

Vamos mostrar no lema a seguir que a aplicação $\mu_{\mathrm{c}}$ é uma medida no espaço mensurável $\left(X, \mathcal{A}_{\mathrm{c}}\right)$; na verdade uma medida cheia. Antes, porém, observamos que como conseqüência imediata da definição de $\mu_{\mathrm{c}}$ temos que se $A \in \mathcal{A}_{\mathrm{c}}$ e se $\mu_{\mathrm{c}}(A)<+\infty$ então $A \in \mathcal{A}$.

Lema 2.2.4. A aplicação $\mu_{\mathrm{c}}: \mathcal{A}_{\mathrm{c}} \rightarrow[0,+\infty]$ definida como em (2.2.3) é uma medida cheia.

DemonstraÇÃo. Mostraremos em primeiro lugar que $\mu_{\mathrm{c}}$ é uma medida. Como $\emptyset \in \mathcal{A}, \mu_{\mathrm{c}}(\emptyset)=\mu(\emptyset)=0$. Seja $\left(A_{n}\right)_{n \geq 1}$ uma seqüência de elementos de 
$\mathcal{A}_{\mathrm{c}}$, dois a dois disjuntos. Se $A_{n} \in \mathcal{A}$ para todo $n \geq 1$ então $\bigcup_{n \geq 1} A_{n} \in \mathcal{A}$ e

$$
\mu_{\mathrm{c}}\left(\bigcup_{n \geq 1} A_{n}\right)=\mu\left(\bigcup_{n \geq 1} A_{n}\right)=\sum_{n \geq 1} \mu\left(A_{n}\right)=\sum_{n \geq 1} \mu_{\mathrm{c}}\left(A_{n}\right)
$$

Vamos supor agora que exista $k \geq 1$ tal que $A_{k} \notin \mathcal{A}$. Então $\mu_{\mathrm{c}}\left(A_{k}\right)=+\infty$, e portanto $\sum_{n \geq 1} \mu_{\mathrm{c}}\left(A_{n}\right)=+\infty$. Seja $E=\bigcup_{n \geq 1} A_{n}$, e suponhamos que $\mu_{\mathrm{c}}(E)<+\infty$. Se $\mu_{\mathrm{c}}(E)<+\infty$ então $E \in \mathcal{A}$ e $\mu(E)<+\infty$; portanto, uma vez que $A_{k} \in \mathcal{A}_{\mathrm{c}}$, temos que $A_{k}=A_{k} \cap E \in \mathcal{A}$, o que contradiz o pressuposto de que $A_{k} \notin \mathcal{A}$. Logo $\mu_{\mathrm{c}}(E)=+\infty$ e $\mu_{\mathrm{c}}\left(\bigcup_{n \geq 1} A_{n}\right)=\sum_{n \geq 1} \mu_{\mathrm{c}}\left(A_{n}\right)$, provando que $\mu_{\mathrm{c}}$ é uma medida em $\mathcal{A}_{\mathrm{c}}$. Vejamos agora que que $\mu_{\mathrm{c}}$ é cheia. Seja $A \subset X$ tal que $A \cap E \in \mathcal{A}_{\mathrm{c}}$ para todo $E \in \mathcal{A}_{\mathrm{c}} \operatorname{com} \mu_{\mathrm{c}}(E)<+\infty$. Queremos mostrar que $A \in \mathcal{A}_{\mathrm{c}}$. Para cada $B \in \mathcal{A} \operatorname{com} \mu(B)<+\infty$ temos $B \in \mathcal{A}_{\mathrm{c}}$ e $\mu_{\mathrm{c}}(B)<+\infty$, e portanto $A \cap B \in \mathcal{A}_{\mathrm{c}}$. Assim, $(A \cap B) \cap B=A \cap B \in \mathcal{A}$ para todo $B \in \mathcal{A} \operatorname{com} \mu(B)<+\infty$, i.e., $A \in \mathcal{A}_{\mathrm{c}}$.

DefiniÇÃo 2.2.5. A medida $\mu_{\mathrm{c}}: \mathcal{A}_{\mathrm{c}} \rightarrow[0,+\infty]$ definida como em (2.2.3) é chamada extensão cheia canônica da medida $\mu: \mathcal{A} \rightarrow[0,+\infty]$.

OBSERVAÇÃo 2.2.6. Se $\mu$ é uma medida cheia em um espaço mensurável $(X, \mathcal{A})$ então $\mathcal{A}_{\mathrm{c}}=\mathcal{A}$ e $\mu_{\mathrm{c}}=\mu$. Com efeito, se $A \in \mathcal{A}_{\mathrm{c}}$ então $A \cap E \in \mathcal{A}$ para todo $E \in \mathcal{A} \operatorname{com} \mu(E)<+\infty$, o que implica $A \in \mathcal{A}$, pois $\mu$ é uma medida cheia. Logo $\mathcal{A}_{\mathrm{c}} \subset \mathcal{A}$, e portanto $\mathcal{A}_{\mathrm{c}}=\mathcal{A}$. Além disso, uma vez que para cada $A \in \mathcal{A}_{\mathrm{c}}$ temos $A \in \mathcal{A}$, segue que $\mu_{\mathrm{c}}(A)=\mu(A)$, ou seja, $\mu_{\mathrm{c}}=\mu$.

Lema 2.2.7. Seja $(X, \mathcal{A}, \mu)$ um espaço de medida. Uma função $f: X \rightarrow \mathbb{R}$ é mensurável em relação a $\mathcal{A}_{\mathrm{c}}$ se e somente se $f \chi_{E}$ é mensurável relativamente a $\mathcal{A}$, para cada $E \in \mathcal{A} \operatorname{com} \mu(E)<+\infty$.

Demonstração. Sejam $f: X \rightarrow \mathbb{R}$ uma função e $E \in \mathcal{A} \operatorname{com} \mu(E)<+\infty$ arbitrário. A função $f \chi_{E}$ é mensurável em relação a $\mathcal{A}$ se e somente se $\left(f \chi_{E}\right)^{-1}(B) \in \mathcal{A}$ para todo $B \in \mathcal{B}(\mathbb{R})$. Mas

$$
\left(f \chi_{E}\right)^{-1}(B)= \begin{cases}f^{-1}(B) \cap E & \text { se } 0 \notin B \\ \left(f^{-1}(B) \cap E\right) \cup E^{c} & \text { se } 0 \in B .\end{cases}
$$

Logo, $f \chi_{E}$ é $\mathcal{A}$-mensurável se e somente se $\left(f^{-1}(B) \cap E\right) \in \mathcal{A}$ para todo $B \in \mathcal{B}(\mathbb{R})$, e portanto, se e somente se $f^{-1}(B) \in \mathcal{A}_{\mathrm{c}}$ para todo $B \in \mathcal{B}(\mathbb{R})$. Daí segue o resultado. 
Corolário 2.2.8. Seja $(X, \mathcal{A}, \mu)$ um espaço de medida. Se $f: X \rightarrow \mathbb{R}$ é $\mathcal{A}_{\mathrm{c}}$-mensurável e $E \subset X$ é $\sigma$-finito para $\mu$ então $f \chi_{E}$ é $\mathcal{A}$-mensurável.

DemonstraçÃo. Se $E \subset X$ é $\sigma$-finito para $\mu$ então existe uma seqüência $\left(F_{n}\right)_{n \geq 1}$ de elementos de $\mathcal{A}$, com $\mu\left(F_{n}\right)<+\infty$ para todo $n \geq 1$, tal que $E=\bigcup_{n \geq 1} F_{n}$. Para cada $n \geq 1$, seja $E_{n}=\bigcup_{k=1}^{n} F_{k}$. Então $\left(E_{n}\right)_{n \geq 1}$ é uma seqüência de elementos de $\mathcal{A}$, com $\mu\left(E_{n}\right)<+\infty$ para todo $n \geq 1$, tal que $E_{n} \nearrow E$. Sendo $f$ uma função mensurável relativamente a $\mathcal{A}_{\mathrm{c}}$, pelo Lema 2.2.7 temos que $f \chi_{E_{n}}$ é $\mathcal{A}$-mensurável para todo $n \geq 1$. Assim, como

$$
f \chi_{E}=\lim _{n \rightarrow+\infty} f \chi_{E_{n}}
$$

segue que $f \chi_{E}$ é mensurável com respeito a $\mathcal{A}$.

Proposição 2.2.9. Seja $(X, \mathcal{A}, \mu)$ um espaço de medida. Para cada $g \in L^{\infty}\left(X, \mathcal{A}_{\mathrm{c}}, \mu_{\mathrm{c}}\right)$ temos $f g \in L^{1}(X, \mathcal{A}, \mu)$ para todo $f \in L^{1}(X, \mathcal{A}, \mu)$ e a fórmula (1.4.3) define um funcional linear limitado $\alpha_{g}$ sobre $L^{1}(X, \mathcal{A}, \mu)$. Reciprocamente, se $g: X \rightarrow \mathbb{R}$ é uma aplicação tal que $f g \in L^{1}(X, \mathcal{A}, \mu)$ para todo $f \in L^{1}(X, \mathcal{A}, \mu)$ e tal que (1.4.3) define um funcional linear limitado $\alpha_{g}$ sobre $L^{1}(X, \mathcal{A}, \mu)$, então g está em $L^{\infty}\left(X, \mathcal{A}_{\mathrm{c}},\left(\mu_{\mathrm{c}}\right)_{\mathrm{lb}}\right)$ e existe $g_{1} \in L^{\infty}\left(X, \mathcal{A}_{\mathrm{c}}, \mu_{\mathrm{c}}\right)$ com $\alpha_{g_{1}}=\alpha_{g}$.

DemonstraÇÃo. Fixemos $g \in L^{\infty}\left(X, \mathcal{A}_{\mathrm{c}}, \mu_{\mathrm{c}}\right)$. Então existe $c \geq 0$ tal que $|g| \leq c \mu_{\mathrm{c}}$-qs, ou seja, existe $B \in \mathcal{A}_{\mathrm{c}} \operatorname{com} \mu_{\mathrm{c}}(B)=0$ tal que $|g(x)| \leq c$ para todo $x \in X \backslash B$. Se $\mu_{\mathrm{c}}(B)=0$ então $B \in \mathcal{A}$ e $\mu(B)=0, \operatorname{logo}|g| \leq c$ $\mu$-qs. Dada $f \in L^{1}(X, \mathcal{A}, \mu)$, pelo Lema 2.1.4, o conjunto $E=f^{-1}(\mathbb{R} \backslash\{0\})$ é $\sigma$-finito para $\mu$. Como $f$ é mensurável em relação a $\mathcal{A}$ e $\mathcal{A} \subset \mathcal{A}_{\mathrm{c}}$ segue que $f$ é $\mathcal{A}_{\mathrm{c}}$-mensurável, e daí $f g$ é mensurável relativamente a $\mathcal{A}_{\mathrm{c}}$. Assim, pelo Corolário 2.2.8, temos que $f g=f g \chi_{E}$ é $\mathcal{A}$-mensurável. Se $|g| \leq c$ $\mu$-qs então $|f g| \leq c|f| \mu$-qs, e portanto $\int_{X}|f g| \mathrm{d} \mu \leq c \int_{X}|f| \mathrm{d} \mu<+\infty$, i.e., $f g \in L^{1}(X, \mathcal{A}, \mu)$. Além disso, para toda $f \in L^{1}(X, \mathcal{A}, \mu)$ temos

$$
\left|\alpha_{g}(f)\right|=\left|\int_{X} f g \mathrm{~d} \mu\right| \leq \int_{X}|f g| \mathrm{d} \mu \leq c \int_{X}|f| \mathrm{d} \mu=c\|f\|_{1},
$$

e portanto (1.4.3) define um funcional linear limitado sobre $L^{1}(X, \mathcal{A}, \mu)$. Reciprocamente, suponhamos que $g: X \rightarrow \mathbb{R}$ é uma aplicação tal que $f g$ está em $L^{1}(X, \mathcal{A}, \mu)$ para toda $f \in L^{1}(X, \mathcal{A}, \mu)$, e tal que a fórmula (1.4.3) define um funcional linear limitado $\alpha_{g}$ sobre $L^{1}(X, \mathcal{A}, \mu)$. Seja $E \in \mathcal{A}$ 
com $\mu(E)<+\infty$ arbitrário. Então $\chi_{E}$ é mensurável em relação a $\mathcal{A}$ e $\int_{X}\left|\chi_{E}\right| \mathrm{d} \mu=\int_{X} \chi_{E} \mathrm{~d} \mu=\mu(E)<+\infty$, ou seja, $\chi_{E} \in L^{1}(X, \mathcal{A}, \mu)$. Portanto $g \chi_{E} \in L^{1}(X, \mathcal{A}, \mu)$ e conseqüentemente $g$ é mensurável com respeito a $\mathcal{A}_{\mathrm{c}}$, pelo Lema 2.2.7. Seja $\delta=\left\|\alpha_{g}\right\|$. Vamos mostrar que $|g| \leq \delta\left(\mu_{\mathrm{c}}\right)_{\mathrm{lb}}$-qs e então teremos $g \in L^{\infty}\left(X, \mathcal{A}_{\mathrm{c}},\left(\mu_{\mathrm{c}}\right)_{\mathrm{lb}}\right)$. Para isso é suficiente mostrar que se $E \in \mathcal{A}_{\mathrm{c}}$ é um subconjunto de $B \stackrel{\text { def }}{=}\{x \in X:|g(x)|>\delta\}$ com $\mu_{\mathrm{c}}(E)<+\infty$, então $\mu_{\mathrm{c}}(E)=0$; daí seguirá que $\left(\mu_{\mathrm{c}}\right)_{\mathrm{lb}}(B)=0$, uma vez que

$$
\left(\mu_{\mathrm{c}}\right)_{\mathrm{lb}}(B)=\sup \left\{\mu_{\mathrm{c}}(E): E \subset B, E \in \mathcal{A}_{\mathrm{c}} \text { e } \mu_{\mathrm{c}}(E)<+\infty\right\} .
$$

Seja $E$ nas condições acima fixado. Então $E \in \mathcal{A}$ e $\mu(E)<+\infty$, e portanto, como já vimos antes, $\chi_{E} \in L^{1}(X, \mathcal{A}, \mu)$. Pela Observação 1.4.32, sendo $g$ mensurável em relação a $\mathcal{A}_{\mathrm{c}}$, a aplicação $\operatorname{sgn}(g)$ também é $\mathcal{A}_{\mathrm{c}}$-mensurável; além disso, $\operatorname{sgn}(g)$ é claramente limitada, portanto $\mu_{\mathrm{c}}$-qs limitada. Isso $\operatorname{mostra}$ que $\operatorname{sgn}(g) \in L^{\infty}\left(X, \mathcal{A}_{\mathrm{c}}, \mu_{\mathrm{c}}\right)$, e $\operatorname{assim} \operatorname{sgn}(g) \chi_{E} \in L^{1}(X, \mathcal{A}, \mu)$. Daí:

$$
\begin{aligned}
\delta \mu(E)= & \int_{X} \delta \chi_{E} \mathrm{~d} \mu=\int_{E} \delta \mathrm{d} \mu \leq \int_{E}|g| \mathrm{d} \mu \\
= & \int_{X}|g| \chi_{E} \mathrm{~d} \mu=\int_{X}\left(\operatorname{sgn}(g) \chi_{E}\right) g \mathrm{~d} \mu=\alpha_{g}\left(\operatorname{sgn}(g) \chi_{E}\right) \\
& \leq \delta\left\|\operatorname{sgn}(g) \chi_{E}\right\|_{1}=\delta \int_{X}\left|\chi_{E}\right| \mathrm{d} \mu=\delta \int_{X} \chi_{E} \mathrm{~d} \mu=\delta \mu(E) .
\end{aligned}
$$

Logo $\int_{X}|g| \chi_{E} \mathrm{~d} \mu=\delta \mu(E)$, i.e., $\int_{X}(|g|-\delta) \chi_{E} \mathrm{~d} \mu=0$. Como $(|g|-\delta) \chi_{E}$ é não negativa, temos que $(|g|-\delta) \chi_{E}=0 \mu$-qs, e portanto

$$
\mu_{\mathrm{c}}(E)=\mu(E) \leq \mu\left(\left\{x \in X:\left((|g|-\delta) \chi_{E}\right)(x) \neq 0\right\}\right)=0,
$$

ou seja, $\mu_{\mathrm{c}}(E)=0$. Vamos mostrar agora que existe $g_{1} \in L^{\infty}\left(X, \mathcal{A}_{\mathrm{c}}, \mu_{\mathrm{c}}\right)$ com $\alpha_{g_{1}}=\alpha_{g}$. Observe que sendo $\left(\mu_{\mathrm{c}}\right)_{\mathrm{lb}}(B)=0$ temos $\mu_{\mathrm{c}}(B)=0$ ou $B$ é um bloco infinito para $\mu_{\mathrm{c}}$. Se $\mu_{\mathrm{c}}(B)=0$ então $|g| \leq \delta \mu_{\mathrm{c}}$-qs, e portanto $g_{1}=g \in L^{\infty}\left(X, \mathcal{A}_{\mathrm{c}}, \mu_{\mathrm{c}}\right)$. Suponhamos, por outro lado, que $B$ seja um bloco infinito para $\mu_{\mathrm{c}}$. Fazendo $g_{1}=g \chi_{X \backslash B}$ temos $g_{1} \in L^{\infty}\left(X, \mathcal{A}_{\mathrm{c}}, \mu_{\mathrm{c}}\right)$; além disso, para $f \in L^{1}(X, \mathcal{A}, \mu)$ segue, pelo Lema 2.1.4, que $\left.f\right|_{B}=0 \mu_{\mathrm{c}}$-qs, e portanto $\left.f\right|_{B}=0 \mu$-qs. Assim, para cada $f \in L^{1}(X, \mathcal{A}, \mu)$ temos

$$
\alpha_{g_{1}}(f)=\int_{X} f g_{1} \mathrm{~d} \mu=\int_{X} f g \chi_{X \backslash B} \mathrm{~d} \mu=\int_{X \backslash B} f g \mathrm{~d} \mu=\int_{X} f g \mathrm{~d} \mu=\alpha_{g}(f),
$$

e isso mostra que $\alpha_{g_{1}}$ e $\alpha_{g}$ são iguais. 
OBSERvaÇÃo 2.2.10. Podemos justificar a última parte da proposição anterior de outra maneira: a versão do diagrama $(2.1 .5)$ para $\left(X, \mathcal{A}_{\mathrm{c}}, \mu_{\mathrm{c}}\right)$ é

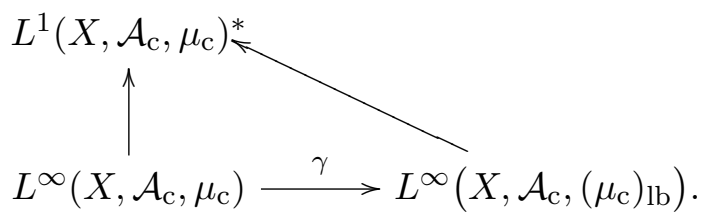

Sabemos que o diagrama é comutativo e a aplicação $\gamma$ é sobrejetora; portanto, se $g \in L^{\infty}\left(X, \mathcal{A}_{\mathrm{c}},\left(\mu_{\mathrm{c}}\right)_{\mathrm{lb}}\right)$ então existe $g_{1} \in L^{\infty}\left(X, \mathcal{A}_{\mathrm{c}}, \mu_{\mathrm{c}}\right)$ tal que $\alpha_{g_{1}}(f)=\alpha_{g}(f)$ para toda $f \in L^{1}\left(X, \mathcal{A}_{\mathrm{c}}, \mu_{\mathrm{c}}\right)$. Mas, pelo Corolário 1.4.67, $L^{1}(X, \mathcal{A}, \mu) \subset L^{1}\left(X, \mathcal{A}_{\mathrm{c}}, \mu_{\mathrm{c}}\right) ; \operatorname{logo} \alpha_{g_{1}}=\alpha_{g}$ em $L^{1}(X, \mathcal{A}, \mu)$.

Visto que a medida $\mu_{\mathrm{c}}$ estende $\mu$, de acordo com a Proposição 1.2.2, a aplicação inclusão de $\mathcal{M}(X, \mathcal{A})$ em $\mathcal{M}\left(X, \mathcal{A}_{\mathrm{c}}\right)$ induz, para cada $p \in[1,+\infty]$, a aplicação linear canônica:

$$
L^{p}(X, \mathcal{A}, \mu) \ni[f]_{\mu} \longmapsto[f]_{\mu_{\mathrm{c}}} \in L^{p}\left(X, \mathcal{A}_{\mathrm{c}}, \mu_{\mathrm{c}}\right) .
$$

Proposição 2.2.11. A aplicação canônica (2.2.4) é uma isometria linear para $p<+\infty$ e uma imersão isométrica para $p=+\infty$.

DemonstraçÃo. Uma vez que $\mathcal{A} \subset \mathcal{A}_{\mathrm{c}}$ e $\mu=\left.\mu_{\mathrm{c}}\right|_{\mathcal{A}}$, com argumento análogo àquele utilizado na demonstração da Proposição 2.2.1, temos que a aplicação (2.2.4) é uma imersão isométrica para todo $p \in[1,+\infty]$. Para $p<+\infty$ afirmamos que (2.2.4) é sobrejetora. Com efeito, se $p \in[1,+\infty$ [ e $f \in L^{p}\left(X, \mathcal{A}_{\mathrm{c}}, \mu_{\mathrm{c}}\right)$ então o conjunto $E=f^{-1}(\mathbb{R} \backslash\{0\})$ é $\sigma$-finito para $\mu_{\mathrm{c}}$, e conseqüentemente para $\mu$. Portanto, pelo Corolário 2.2.8, $f=f \chi_{E}$ é mensurável em relação a $\mathcal{A}$. Como $\left\|[f]_{\mu}\right\|_{p}=\left\|[f]_{\mu_{\mathrm{c}}}\right\|_{p}<+\infty$, segue que $f \in L^{p}(X, \mathcal{A}, \mu)$.

Seja $(X, \mathcal{A}, \mu)$ é um espaço de medida. Para $p, q \in[1,+\infty] \operatorname{com} \frac{1}{p}+\frac{1}{q}=1$ é comutativo o diagrama:

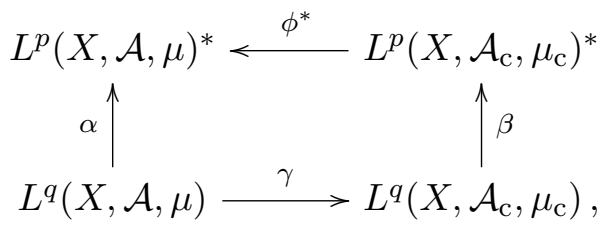

no qual $\alpha$ e $\beta$ são $(q, p)$-aplicações de Riesz (1.4.2) para os espaços $(X, \mathcal{A}, \mu)$ e $\left(X, \mathcal{A}_{\mathrm{c}}, \mu_{\mathrm{c}}\right), \phi^{*}$ é a transposta da aplicação (2.2.4), e $\gamma$ é a versão da aplicação 
(2.2.4) para $L^{q}$. De fato, como $\mathcal{A} \subset \mathcal{A}_{\mathrm{c}}$ e $\mu=\left.\mu_{\mathrm{c}}\right|_{\mathcal{A}}$, com argumento similar àquele usado para justificar a comutatividade do diagrama (2.2.2), segue que o diagrama (2.2.5) comuta.

\subsection{Medidas perfeitas}

Proposição 2.3.1. Seja $(X, \mathcal{A})$ um espaço mensurável. Dada uma me$\operatorname{dida} \mu: \mathcal{A} \rightarrow[0,+\infty]$, temos:

(a) se $\mu$ é completa então $\mu_{\mathrm{c}}$ também é completa;

(b) se $\mu$ é cheia então $\mu_{\mathrm{lb}}$ também é cheia;

(c) se $\mu$ é cheia e completa então $\mu_{\mathrm{lb}}$ também é (cheia e) completa.

DEMONSTRAÇÃO.

- Prova de (a).

Sejam $B \in \mathcal{A}_{\mathrm{c}}$ com $\mu_{\mathrm{c}}(B)=0$ e $A \subset B$ qualquer. Então $B \in \mathcal{A}$ e $\mu(B)=0$, o que implica $A \in \mathcal{A}$, pois $\mu$ é completa. Logo $A \in \mathcal{A}_{\mathrm{c}}$, e portanto $\mu_{\mathrm{c}}$ é completa.

- Prova de (b).

Seja $A$ um subconjunto de $X$ tal que $A \cap B \in \mathcal{A}$ para cada $B \in \mathcal{A}$ com $\mu_{\mathrm{lb}}(B)<+\infty$. Seja $E \in \mathcal{A} \operatorname{com} \mu(E)<+\infty$ arbitrário. Pelo item (b) da Proposição 2.1.7 temos $\mu_{\mathrm{lb}}(E)=\mu(E)<+\infty$. Logo $A \cap E \in \mathcal{A}$, e dado que $\mu$ é uma medida cheia, segue que $A \in \mathcal{A}$. Assim, $\mu_{\mathrm{lb}}$ é uma medida cheia.

- Prova de (c).

Fixemos $B \in \mathcal{A} \operatorname{com} \mu_{\mathrm{lb}}(B)=0$, e seja $A$ um subconjunto arbitrário de $B$. De $\mu_{\mathrm{lb}}(B)=0$ temos que $\mu(B)=0$ ou $B$ é um bloco infinito para $\mu$. Se $\mu(B)=0$ então $A \in \mathcal{A}$, pois $\mu$ é completa. Se $B$ é um bloco infinito para $\mu$ então, pelo Lema 2.1.3, $\mu(B \cap E)=0$ para cada $E \in \mathcal{A}$ com $\mu(E)<+\infty$. Logo, como $\mu$ é completa, $A \cap E \in \mathcal{A}$ para cada $E \in \mathcal{A} \operatorname{com} \mu(E)<+\infty$. Assim, sendo $\mu$ uma medida cheia, segue que $A \in \mathcal{A}$. Portanto $\mu_{\mathrm{lb}}$ é uma medida completa.

DefiniçÃo 2.3.2. Uma medida $\mu: \mathcal{A} \rightarrow[0,+\infty]$ em um espaço mensurável $(X, \mathcal{A})$ é chamada de perfeita se ela é simultaneamente completa, cheia e livre de blocos. 
Dada uma medida $\mu: \mathcal{A} \rightarrow[0,+\infty]$, denotamos por $\mu_{\mathfrak{p}}: \mathcal{A}_{\mathfrak{p}} \rightarrow[0,+\infty]$ a versão livre de blocos da extensão cheia canônica do completamento de $\mu$, ou seja, $\mu_{\mathfrak{p}}=\left((\bar{\mu})_{\mathrm{c}}\right)_{\mathrm{lb}}$ e $\mathcal{A}_{\mathfrak{p}}=(\overline{\mathcal{A}})_{\mathrm{c}}$. É claro que $\mu_{\mathfrak{p}}$ é uma medida, pois dada uma medida, as ações "completar", "encher"e "livrar de blocos" geram uma nova medida. Pelo item (a) da Proposição 2.3.1 temos que $(\bar{\mu})_{\mathrm{c}}$ é completa, e portanto, pelo item (c) dessa proposição, $\left((\bar{\mu})_{\mathrm{c}}\right)_{\mathrm{lb}}$ é cheia e completa. Assim, $\mu_{\mathfrak{p}}$ é cheia, completa e livre de blocos, ou seja, perfeita.

DefiniÇÃo 2.3.3. A medida $\mu_{\mathfrak{p}}: \mathcal{A}_{\mathfrak{p}} \rightarrow[0,+\infty]$ definida acima chama-se versão perfeita da medida $\mu$.

Os dois exemplos que seguem evidenciam o fato de que não é possível permutar a ordem "encher, livrar de blocos" nem a ordem "completar, encher".

Exemplo 2.3.4. Sejam $X$ um conjunto não enumerável e $\mathcal{A}$ a $\sigma$-álgebra consistindo dos subconjuntos de $X$ que são enumeráveis ou têm complementar em $X$ enumerável. Sejam $S$ um subconjunto de $X$ que não está em $\mathcal{A}$ e $\mu: \mathcal{A} \rightarrow[0,+\infty]$ a aplicação definida por: para cada $A \in \mathcal{A}, \mu(A)$ é o número de elementos de $A \cap S$. É fácil ver que $\mu$ é uma medida em $\mathcal{A}$. Observe que se $A \in \mathcal{A}$ tem complementar em $X$ enumerável então $A \cap S=S \backslash(X \backslash A)$ é não enumerável e portanto $\mu(A)=+\infty$. A medida $\mu$ é claramente completa, uma vez que se $A \in \mathcal{A}$ tem medida nula então $A$ é enumerável e portanto cada subconjunto de $A$ está em $\mathcal{A}$. A medida $\mu$ é também livre de blocos, pois se $A \in \mathcal{A}$ é tal que $\mu(A)=+\infty$ então $A \cap S$ é um conjunto infinito e, para cada $x \in A \cap S$, o conjunto unitário $\{x\}$ é um subconjunto mensurável de $A \operatorname{com} \mu(\{x\})=1$. A medida $\mu$ não é cheia; além disso, a $\sigma$-álgebra $\mathcal{A}_{\mathrm{c}}$ coincide com $\mathcal{P}(X)$, o conjunto das partes de $X$. Com efeito, se $A \in \mathcal{P}(X)$ é um subconjunto arbitrário de $X$ e se $E \in \mathcal{A}$ tem medida finita então $S \backslash(X \backslash E)=E \cap S$ é finito. Portanto $E$ é enumerável e conseqüentemente ( $A \cap E$ é também enumerável) $A \cap E \in \mathcal{A}$, o que mostra que $A \in \mathcal{A}_{\mathrm{c}}$. Consideremos agora a extensão cheia canônica $\mu_{\mathrm{c}}: \mathcal{P}(X) \rightarrow[0,+\infty]$ de $\mu$. Afirmamos que o conjunto $X \backslash S$ é um bloco infinito para $\mu_{\mathrm{c}}$. De fato, uma vez que $X \backslash S$ não está em $\mathcal{A}$ temos $\mu_{\mathrm{c}}(X \backslash S)=+\infty$. Agora, dado um subconjunto $A$ de $X \backslash S$, se $A$ não é enumerável então $A \notin \mathcal{A}$ (pois $S \subset X \backslash A$, e portanto $X \backslash A$ é não enumerável), e assim $\mu_{\mathrm{c}}(A)=+\infty$. Se $A$ é enumerável então $A \in \mathcal{A}$, e uma vez que $A \cap S=\emptyset$ temos $\mu_{\mathrm{c}}(A)=\mu(A)=0$. 
O Exemplo 2.3.4 ilustra o fato de que a extensão cheia canônica de uma medida livre de blocos (e completa) não é em geral livre de blocos. Isso também ilustra o fato de que a versão livre de blocos da extensão cheia canônica de uma medida não é, em geral, o mesmo que a extensão cheia canônica de sua versão livre de blocos, ou seja, $\left(\mu_{\mathrm{lb}}\right)_{\mathrm{c}} \neq\left(\mu_{\mathrm{c}}\right)_{\mathrm{lb}}$ (até mesmo quando ambas, $\left(\mu_{\mathrm{lb}}\right)_{\mathrm{c}}$ e $\left(\mu_{\mathrm{c}}\right)_{\mathrm{lb}}$ estão definidas sobre a mesma $\sigma$-álgebra).

Exemplo 2.3.5. Seja $\Lambda$ um conjunto não enumerável arbitrário e seja $X=[0,1] \times \Lambda$. Considere a $\sigma$-álgebra $\mathcal{A}$ consistindo de todos os subconjuntos $A$ de $X$ tais que a $\lambda$-ésima linha

$$
A^{\lambda}=\{x \in[0,1]:(x, \lambda) \in A\}
$$

está em $\left.\mathcal{B}(\mathbb{R})\right|_{[0,1]}$ (i.e., se é um subconjunto boreleano de $\left.[0,1]\right)$, para todo $\lambda \in \Lambda$. A aplicação $\mu: \mathcal{A} \rightarrow[0,+\infty]$, definida por

$$
\mu(A)= \begin{cases}\sum_{\lambda \in \Lambda} \mathfrak{m}\left(A^{\lambda}\right) & \text { se o conjunto }\left\{\lambda \in \Lambda: A^{\lambda} \neq \emptyset\right\} \text { é enumerável } \\ +\infty & \text { caso contrário, }\end{cases}
$$

é uma medida em $\mathcal{A}$. De modo análogo ao que foi feito no Exemplo 2.1.6 mostra-se que, de fato, $\mathcal{A}$ é uma $\sigma$-álgebra de partes de $X$ e que $\mu$ é uma medida em $\mathcal{A}$. A medida $\mu$ não é livre de blocos; com efeito, se $A \in \mathcal{A}$ tem uma quantidade não enumerável de linhas não vazias, e se todas as linhas de $A$ têm medida de Lebesgue igual a zero, então $A$ é um bloco infinito para $\mu$. De fato, $\mu(A)=+\infty$ e para cada $B \subset A, B \in \mathcal{A}$, temos $\mu(B)=+\infty$ se o número de linhas não vazias de $B$ é não enumerável, e $\mu(B)=\sum_{\lambda \in \Lambda} \mathfrak{m}\left(B^{\lambda}\right)=0$, caso contrário. A medida $\mu$ é cheia; com efeito, dado $A \in \mathcal{A}_{\mathrm{c}}$ então, para cada $\lambda \in \Lambda$, o conjunto $[0,1] \times\{\lambda\} \in \mathcal{A}$ tem medida finita e portanto

$$
A \cap([0,1] \times\{\lambda\}) \in \mathcal{A} .
$$

Mas a $\lambda$-ésima linha de $A \cap([0,1] \times\{\lambda\})$ é igual à $\lambda$-ésima linha $A^{\lambda}$ de $A$, e portanto $A^{\lambda}$ é boreleano de $[0,1]$ para todo $\lambda \in \Lambda$, i.e., $A \in \mathcal{A}$. A medida $\mu$ não é completa e o completamento $\bar{\mu}: \overline{\mathcal{A}} \rightarrow[0,+\infty]$ de $\mu$ é definido na $\sigma$-álgebra $\overline{\mathcal{A}}$ consistindo dos subconjuntos $A \subset X$ tais que $A^{\lambda}$ é um subconjunto Lebesgue mensurável de $[0,1]$ para todo $\lambda \in \Lambda$ e tal que é enumerável o conjunto

$$
\mathfrak{A} \stackrel{\text { def }}{=}\left\{\lambda \in \Lambda: A^{\lambda} \text { não é boreleano }\right\}
$$


ou seja, um conjunto é $\overline{\mathcal{A}}$-mensurável se todas as suas linhas são Lebesgue mensuráveis e apenas uma quantidade enumerável delas não é boreleana. De fato, se $A \in \overline{\mathcal{A}}$ então $A=B \cup N$, com $N \subset M, B, M \in \mathcal{A}$ e $\mu(M)=0$. Para cada $\lambda \in \Lambda$ temos $A^{\lambda}=B^{\lambda} \cup N^{\lambda}$, com $N^{\lambda} \subset M^{\lambda}, B^{\lambda}$ e $M^{\lambda}$ boreleanos e $\mathfrak{m}\left(M^{\lambda}\right)=0$, e assim $A^{\lambda}$ é Lebesgue mensurável (pois, $\mathcal{L}=\overline{\mathcal{B}(\mathbb{R})}$ ). Além disso, o conjunto $\mathfrak{A}$ está contido no conjunto

$$
\mathfrak{B} \stackrel{\text { def }}{=}\left\{\lambda \in \Lambda: M^{\lambda} \neq \emptyset\right\},
$$

que é enumerável, visto que $\mu(M)$ é finita. Reciprocamente, suponhamos que $A \subset X$ é tal que todas as linhas de $A$ são Lebesgue mensuráveis, com apenas uma quantidade enumerável de não boreleanos. Para cada $\lambda \in \Lambda$, existem $B(\lambda), M(\lambda)$ boreleanos de $[0,1]$, com $\mathfrak{m}(M(\lambda))=0$, tais que

$$
A^{\lambda}=B(\lambda) \cup N(\lambda)
$$

para algum $N(\lambda) \subset M(\lambda)$, e $N(\lambda)=M(\lambda)=\emptyset$ se $A^{\lambda} \in \mathcal{B}(\mathbb{R})$. Sejam

$$
\begin{aligned}
& B=\bigcup_{\lambda \in \Lambda}(B(\lambda) \times\{\lambda\}), \\
& N=\bigcup_{\lambda \in \Lambda}(N(\lambda) \times\{\lambda\}), \\
& M=\bigcup_{\lambda \in \Lambda}(M(\lambda) \times\{\lambda\}),
\end{aligned}
$$

Observe que para cada $\lambda \in \Lambda$ temos $B^{\lambda}=B(\lambda), N^{\lambda}=N(\lambda)$ e $M^{\lambda}=M(\lambda)$. Então $B, M \in \mathcal{A}$ e $\mu(M)=0$, uma vez que o conjunto $\mathfrak{B}$ é enumerável (pois $\mathfrak{A}$ é enumerável). Além disso, claramente $N \subset M$ e

$$
\begin{aligned}
B \cup N=\left(\bigcup_{\lambda \in \Lambda}(\right. & B(\lambda) \times\{\lambda\})) \cup\left(\bigcup_{\lambda \in \Lambda}(N(\lambda) \times\{\lambda\})\right) \\
& =\bigcup_{\lambda \in \Lambda}((B(\lambda) \cup N(\lambda)) \times\{\lambda\})=\bigcup_{\lambda \in \Lambda}\left(A^{\lambda} \times\{\lambda\}\right)=A,
\end{aligned}
$$

o que mostra que $A \in \overline{\mathcal{A}}$. Afirmamos que a medida $\bar{\mu}$ não é cheia. Em primeiro lugar, note que se $E \in \overline{\mathcal{A}}$ é tal que $\bar{\mu}(E)<+\infty$ então $E$ possui uma quantidade enumerável de linhas não vazias; com efeito, se $E$ tem uma quantidade não enumerável de linhas não vazias, seja $E_{0}$ o conjunto contendo exatamente um ponto de cada linha de $E$. Então $E_{0} \in \mathcal{A}, E_{0} \subset E \mathrm{e}$ $\bar{\mu}\left(E_{0}\right)=\mu\left(E_{0}\right)=+\infty$, e assim $\bar{\mu}(E)=+\infty$. Agora, seja $S$ um subconjunto Lebesgue mensurável de $[0,1]$ que não é boreleano. O conjunto $A=S \times \Lambda$ 
não está em $\overline{\mathcal{A}}$, pois $A^{\lambda} \notin \mathcal{B}(\mathbb{R})$ para uma quantidade não enumerável de índices $\lambda \in \Lambda$. Nós afirmamos que $A$ está em $(\overline{\mathcal{A}})_{\mathrm{c}}$. De fato, se $E \in \overline{\mathcal{A}}$ é tal que $\bar{\mu}(E)<+\infty$ então $E$ tem apenas uma quantidade enumerável de linhas não vazias, e portanto $A \cap E$ possui apenas uma quantidade enumerável de linhas que não estão em $\mathcal{B}(\mathbb{R})$ (pois tem, é claro, uma quantidade enumerável de linhas não vazias). Além disso, todas as linhas de $A \cap E$ são Lebesgue mensuráveis e portanto $A \cap E \in \overline{\mathcal{A}}$.

O Exemplo 2.3.5 ilustra o fato de que o completamento de uma medida cheia pode não ser uma medida cheia, e que a extensão cheia canônica do completamento de uma medida pode não coincidir com o completamento da extensão cheia canônica dessa medida, i.e., pode ocorrer $(\bar{\mu})_{\mathrm{c}} \neq \overline{\left(\mu_{\mathrm{c}}\right)}$.

Seja $(X, \mathcal{A}, \mu)$ um espaço de medida. Observamos que $\mathcal{A} \subset \overline{\mathcal{A}} \subset(\overline{\mathcal{A}})_{\mathrm{c}}=\mathcal{A}_{\mathfrak{p}}$ e, para cada $p \in[1,+\infty]$, a aplicação inclusão de $\mathcal{M}(X, \mathcal{A})$ em $\mathcal{M}\left(X, \mathcal{A}_{\mathfrak{p}}\right)$ induz a aplicação linear:

$$
L^{p}(X, \mathcal{A}, \mu) \ni[f]_{\mu} \longmapsto[f]_{\mu_{\mathfrak{p}}} \in L^{p}\left(X, \mathcal{A}_{\mathfrak{p}}, \mu_{\mathfrak{p}}\right)
$$

que é exatamente a composição das aplicações $(2.2 .1),(2.2 .4)$ e (2.1.2). Com efeito, para cada $f \in L^{p}(X, \mathcal{A}, \mu)$, temos:

$$
[f]_{\mu} \stackrel{(2.2 .1)}{\longmapsto}[f]_{\bar{\mu}} \stackrel{(2.2 .4)}{\longmapsto}[f]_{(\bar{\mu})_{\mathrm{c}}} \stackrel{(2.1 .2)}{\longmapsto}[f]_{\left((\bar{\mu})_{\mathrm{c}}\right)_{\mathrm{lb}}}=[f]_{\mu_{\mathfrak{p}}} .
$$

Proposição 2.3.6. A aplicação (2.3.1) é uma isometria linear para $p \in[1,+\infty[$.

Demonstração. Segue das Proposições 2.1.9, 2.2.1 e 2.2.11.

Combinando os diagramas comutativos (2.2.2), (2.2.5) e (2.1.4) nós obtemos um novo diagrama comutativo:

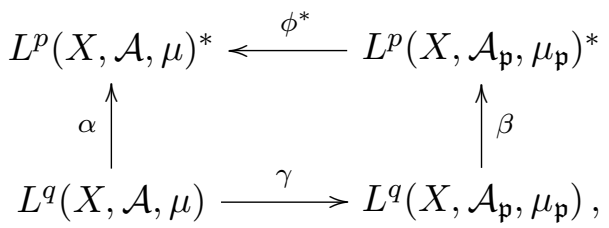

no qual $\alpha$ e $\beta$ são $(q, p)$-aplicações de Riesz (1.4.2) para os espaços $(X, \mathcal{A}, \mu) \mathrm{e}$ $\left(X, \mathcal{A}_{\mathfrak{p}}, \mu_{\mathfrak{p}}\right), \phi^{*}$ é a transposta da aplicação (2.3.1) e $\gamma$ é a versão da aplicação (2.3.1) para $L^{q}$. Combinando os diagramas mencionados acima, e lembrando 
que $\mu_{\mathfrak{p}}=\left((\bar{\mu})_{\mathrm{c}}\right)_{\mathrm{lb}}$ e $\mathcal{A}_{\mathfrak{p}}=(\overline{\mathcal{A}})_{\mathrm{c}}$, obtemos o diagrama a seguir, no qual vemos que, de fato, o diagrama (2.3.2) comuta:

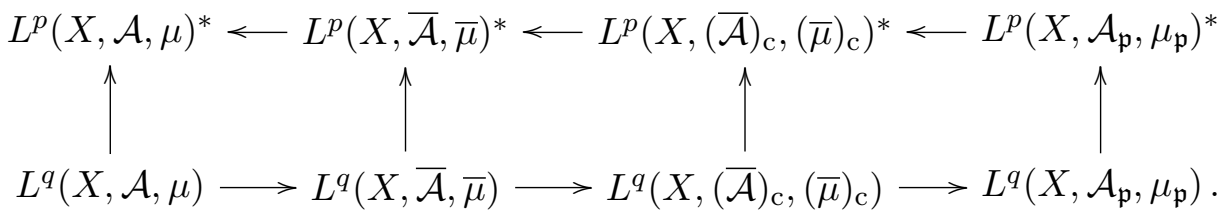

Para medidas perfeitas a aplicação de Riesz 1.4.4 é sempre uma imersão isométrica (Proposição 2.1.5), o que lhe dá "melhores chances" de ser uma isometria linear. Quando passamos de uma medida $\mu$ para a sua versão perfeita $\mu_{\mathfrak{p}}$, nós não modificamos (a menos de identificação isométrica natural) os espaços $L^{p}$ para $p<+\infty$, e tornamos o espaço $L^{\infty}$ mais conveniente para a bijetividade da aplicação de Riesz; livramos-nos do núcleo da aplicação de Riesz tomando um quociente de $L^{\infty}$ (Proposição 2.1.10) e estendemos $L^{\infty}$ para o mais amplo espaço possível de aplicações $g$ que correspondem a funcionais $\alpha_{g}$ em $L^{1}$ (Proposição 2.2.9). Apesar disso, eliminamos apenas os obstáculos "ingênuos" para a bijetividade da aplicação de Riesz 1.4.4, ou seja, embora espaços de medida perfeita favoreçam tal bijetividade, não é necessariamente isso o que ocorre. Na próxima seção apresentamos um espaço de medida perfeita para o qual a aplicação de Riesz não é sobrejetora.

\subsection{Um contra-exemplo não trivial para a bijetividade da Aplicação de Riesz}

Exemplo 2.4.1. Dados dois conjuntos $C_{1}, C_{2}$ e $A \subset X=C_{1} \times C_{2}$, para cada $y \in C_{2}$ denotamos por $A^{y}$ a linha $\left\{x \in C_{1}:(x, y) \in A\right\}$ e para cada $x \in C_{1}$ denotamos por $A_{x}$ a coluna $\left\{y \in C_{2}:(x, y) \in A\right\}$. Suponhamos que $C_{1}$ e $C_{2}$ sejam não enumeráveis. Seja $\mathcal{A}$ a coleção consistindo dos subconjuntos $A$ de $X$ tais que:

- ou $A^{y}$ ou $C_{1} \backslash A^{y}$ é enumerável, para todo $y \in C_{2}$;

- ou $A_{x}$ ou $C_{2} \backslash A_{x}$ é enumerável, para todo $x \in C_{1}$.

Afirmamos que $\mathcal{A}$ é uma $\sigma$-álgebra de partes de $X$. De fato, sejam

$$
\begin{aligned}
& \mathcal{A}_{1}=\left\{A \subset C_{1}: A \text { é enumerável ou } C_{1} \backslash A \text { é enumerável }\right\} \\
& \mathcal{A}_{2}=\left\{A \subset C_{2}: A \text { é enumerável ou } C_{2} \backslash A \text { é enumerável }\right\}
\end{aligned}
$$


$\sigma$-álgebras de partes de $C_{1}$ e $C_{2}$ respectivamente (Exemplo 1.4.8). Para cada $y \in C_{2}$ e para cada $x \in C_{1}$ sejam $i^{y}: C_{1} \rightarrow X$ e $i_{x}: C_{2} \rightarrow X$ aplicações dadas por $i^{y}(x)=(x, y)$ para todo $x \in C_{1}$, e $i_{x}(y)=(x, y)$ para todo $y \in C_{2}$. Uma vez que

$$
\begin{array}{r}
\mathcal{A}=\left\{A \subset X: A^{y} \in \mathcal{A}_{1} \text { para todo } y \in C_{2} \text { e } A_{x} \in \mathcal{A}_{2} \text { para todo } x \in C_{1}\right\} \\
=\left\{A \subset X:\left(i^{y}\right)^{-1}(A) \in \mathcal{A}_{1} \text { para todo } y \in C_{2} \text { e }\left(i_{x}\right)^{-1}(A) \in \mathcal{A}_{2}\right. \\
\text { para todo } \left.x \in C_{1}\right\},
\end{array}
$$

segue, pela Proposição 1.4.11, que $\mathcal{A}$ é a $\sigma$-álgebra co-induzida em $X$ pelas famílias de funções $\left(i^{y}\right)_{y \in C_{2}}$ e $\left(i_{x}\right)_{x \in C_{1}}$.

Dados $x \in C_{1}$ e $y \in C_{2}$, sejam $\mu_{x}: \mathcal{A} \rightarrow[0,+\infty]$ e $\mu^{y}: \mathcal{A} \rightarrow[0,+\infty]$ aplicações definidas por:

$$
\begin{aligned}
& \mu_{x}(A)= \begin{cases}0 & \text { se } A_{x} \text { é enumerável } \\
1 & \text { se } C_{2} \backslash A_{x} \text { é enumerável, }\end{cases} \\
& \mu^{y}(A)= \begin{cases}0 & \text { se } A^{y} \text { é enumerável } \\
1 & \text { se } C_{1} \backslash A^{y} \text { é enumerável, }\end{cases}
\end{aligned}
$$

para todo $A \in \mathcal{A}$. Para $i=1,2$ seja $\mu_{i}: \mathcal{A}_{i} \rightarrow[0,+\infty]$ a aplicação dada por:

$$
\mu_{i}(B)= \begin{cases}0 & \text { se } B \text { é enumerável } \\ 1 & \text { se } C_{i} \backslash B \text { é enumerável }\end{cases}
$$

para todo $B \in \mathcal{A}_{i}$. Pela Proposição 1.4.24, $\mu_{i}$ é uma medida em $\mathcal{A}_{i}, i=1,2$. Assim, fixados $x \in C_{1}$ e $y \in C_{2}$, para todo $A \in \mathcal{A}$ temos

$$
\mu_{x}(A)=\mu_{2}\left(A_{x}\right)=\mu_{2}\left(\left(i_{x}\right)^{-1}(A)\right) \text { e } \mu^{y}(A)=\mu_{1}\left(A^{y}\right)=\mu_{1}\left(\left(i^{y}\right)^{-1}(A)\right) .
$$

Logo, pelo item (a) da Proposição 1.4.46, as aplicações $\mu_{x}$ e $\mu^{y}$ são medidas em $\mathcal{A}$ para todo $x \in C_{1}$ e para todo $y \in C_{2}$.

Seja, por fim, $\mu: \mathcal{A} \rightarrow[0,+\infty]$ a aplicação definida por:

$$
\mu(A)=\sum_{x \in C_{1}} \mu_{x}(A)+\sum_{y \in C_{2}} \mu^{y}(A)
$$

para todo $A \in \mathcal{A}$. Então, pela Proposição 1.4 .20 (e Corolário 1.4.21), a aplicação $\mu$ é uma medida em $\mathcal{A}$.

Lema 2.4.2. A medida $\mu$ definida acima é perfeita. 


\section{DEMONSTRAÇÃO.}

- $\mu$ é completa.

Seja $A \in \mathcal{A} \operatorname{com} \mu(A)=0$. Então $A_{x}$ e $A^{y}$ são enumeráveis para todo $x \in C_{1}$ e para todo $y \in C_{2}$. Portanto, se $B$ é um subconjunto de $A$, então $B_{x}$ e $B^{y}$ são também enumeráveis para todo $x \in C_{1}$ e para todo $y \in C_{2}$. Segue que $B$ está em $\mathcal{A}$.

- $\mu$ é cheia.

Seja $A \subset X$ tal que $A \cap B \in \mathcal{A}$ para todo $B \in \mathcal{A} \operatorname{com} \mu(B)<+\infty$. Seja $y_{o} \in C_{2}$ fixado. Afirmamos que o conjunto $E=C_{1} \times\left\{y_{o}\right\}$ está em $\mathcal{A}$ e $\mu(E)=1$. Com efeito, $E_{x}=\left\{y_{o}\right\}$ para todo $x \in C_{1}, E^{y}=C_{1}$ se $y=y_{o}$ e $E^{y}=\emptyset$ se $y \neq y_{o}$. Portanto $E_{x}$ é enumerável para todo $x \in C_{1}$ e, ou $E^{y}$ ou $C_{1} \backslash E^{y}$ é enumerável para todo $y \in C_{2} ; \operatorname{logo} E \in \mathcal{A}$. Além disso, $\mu_{x}(E)=0$ para todo $x \in C_{1}, \mu^{y}(E)=0$ para todo $y \in C_{2} \backslash\left\{y_{o}\right\}$ e $\mu^{y_{o}}(E)=1$. Assim,

$$
\mu(E)=\sum_{x \in C_{1}} \mu_{x}(E)+\sum_{y \in C_{2} \backslash\left\{y_{o}\right\}} \mu^{y}(E)+\mu^{y_{o}}(E)=1 .
$$

Portanto $A \cap E \in \mathcal{A}$, o que implica que $(A \cap E)^{y_{o}}$ é enumerável ou tem complementar em $C_{1}$ enumerável. Mas

$$
\begin{aligned}
(A \cap E)^{y_{o}} & =\left\{x \in C_{1}:\left(x, y_{o}\right) \in A \cap E\right\} \\
& =\left\{x \in C_{1}:\left(x, y_{o}\right) \in A\right\} \cap\left\{x \in C_{1}:\left(x, y_{o}\right) \in E\right\}=A^{y_{o}} \cap C_{1}=A^{y_{o}} .
\end{aligned}
$$

Logo, ou $A^{y}$ é enumerável ou $C_{1} \backslash A^{y}$ é enumerável, para todo $y \in C_{2}$. Analogamente, fazendo $E=\{x\} \times C_{2}$ para cada $x \in C_{1}$, mostra-se que $A_{x}$ é enumerável ou $C_{2} \backslash A_{x}$ é enumerável, para todo $x \in C_{1}$. Portanto $A \in \mathcal{A}$, o que mostra que $\mu$ é uma medida cheia.

- $\mu$ é livre de blocos.

Se $A \in \mathcal{A}$ é tal que $\mu(A)=+\infty$ então ou existe $x_{o} \in C_{1}$ com $\mu_{x_{o}}(A)=1$ ou existe $y_{o} \in C_{2} \operatorname{com} \mu^{y_{o}}(A)=1$. Suponhamos que exista $x_{o} \in C_{1} \operatorname{com} \mu_{x_{o}}(A)=1$. Se $E \stackrel{\text { def }}{=}\left\{x_{o}\right\} \times A_{x_{o}} \subset A$ então $E \in \mathcal{A}$, uma vez que $E^{y}=\left\{x_{o}\right\}$ para todo $y \in C_{2}$, e para todo $x \in C_{1}$ temos $E_{x}=\emptyset$ se $x \neq x_{o}$ e $E_{x_{o}}=A_{x_{o}}$. Assim, $\mu^{y}(E)=0$ para todo $y \in C_{2}, \mu_{x}(E)=0$ para todo $x \in C_{1} \backslash\left\{x_{o}\right\}$ e $\mu_{x_{o}}(E)=1$, pois sendo $\mu_{x_{o}}(A)=1$ temos 
que $C_{2} \backslash E_{x_{o}}=C_{2} \backslash A_{x_{o}}$ é enumerável. Portanto

$$
\mu(E)=\mu_{x_{o}}(E)+\sum_{x \in C_{1} \backslash\left\{x_{o}\right\}} \mu_{x}(E)+\sum_{y \in C_{2}} \mu^{y}(E)=1 .
$$

De modo análogo, se existir $y_{o} \in C_{2} \operatorname{com} \mu^{y_{o}}(A)=1$ então $E=A^{y_{o}} \times\left\{y_{o}\right\}$ está em $\mathcal{A}$ e $\mu(E)=1$. Portanto $A$ não é um bloco infinito para $\mu$.

Lema 2.4.3. Seja $(X, \mathcal{A}, \mu)$ o espaço de medida definido como no Exemplo 2.4.1. Se a aplicação de Riesz (1.4.4) desse espaço é um isomorfismo então existe um subconjunto $R$ de $X=C_{1} \times C_{2}$ tal que $R^{y}$ é enumerável para todo $y \in C_{2}$ e $C_{2} \backslash R_{x}$ é enumerável para todo $x \in C_{1}$.

DemonstraÇão. Considere a medida $\nu: \mathcal{A} \rightarrow[0,+\infty]$ definida por:

$$
\nu(A)=\sum_{x \in C_{1}} \mu_{x}(A)
$$

para todo $A \in \mathcal{A}$. Uma vez que $\nu(A) \leq \mu(A)$ para todo $A \in \mathcal{A}$, a integração com respeito a $\nu$ define um funcional linear limitado sobre $L^{1}(X, \mathcal{A}, \mu)$; mais explicitamente, a aplicação $\alpha(f)=\int_{X} f \mathrm{~d} \nu$ é um funcional linear sobre $L^{1}(X, \mathcal{A}, \mu)$ com $\|\alpha\| \leq 1$. De fato, $\alpha$ é claramente linear e para cada $f \in L^{1}(X, \mathcal{A}, \mu)$, como $\nu \leq \mu$, pela Proposição 1.4.58, temos

$$
\alpha(f)=\int_{X} f \mathrm{~d} \nu \leq \int_{X}|f| \mathrm{d} \nu \leq \int_{X}|f| \mathrm{d} \mu=\|f\|_{1}<+\infty .
$$

Isso mostra que $\alpha$ está bem definida e que $\|f\|_{1} \leq 1$, pois

$$
\|\alpha\|=\inf \left\{c \geq 0:|\alpha(f)| \leq c\|f\|_{1} \text { para toda } f \in L^{1}(X, \mathcal{A}, \mu)\right\} .
$$

Se a aplicação de Riesz (1.4.4) do espaço $(X, \mathcal{A}, \mu)$ é um isomorfismo então existe $g \in L^{\infty}(X, \mathcal{A}, \mu)$ com $\alpha_{g}=\alpha$. Seja $y \in C_{2}$ fixado e consideremos o conjunto $E=C_{1} \times\{y\}$. Sejam ainda $\mathcal{A}^{\prime}=\left.\mathcal{A}\right|_{E}$ a $\sigma$-álgebra induzida por $\mathcal{A}$ em $E$ e $\mu^{\prime}=\left.\mu\right|_{\mathcal{A}^{\prime}}$. Então a aplicação de Riesz

$$
L^{\infty}\left(E, \mathcal{A}^{\prime}, \mu^{\prime}\right) \longrightarrow L^{1}\left(E, \mathcal{A}^{\prime}, \mu^{\prime}\right)^{*}
$$

é injetora e leva $\left.g\right|_{E}$ na restrição de $\alpha$ ao espaço $L^{1}\left(E, \mathcal{A}^{\prime}, \mu^{\prime}\right)$. Com efeito, a aplicação de Riesz do espaço $\left(E, \mathcal{A}^{\prime}, \mu^{\prime}\right)$ é injetora porque $\mu$ é uma medida livre de blocos (Proposição 2.1.5) e, pela Observação 1.4.85, leva $\left.g\right|_{E} \mathrm{em}$ $\left.\alpha_{g}\right|_{L^{1}\left(E, \mathcal{A}^{\prime}, \mu^{\prime}\right)}=\left.\alpha\right|_{L^{1}\left(E, \mathcal{A}^{\prime}, \mu^{\prime}\right)}$. Note que para todo $x \in C_{1}$ temos $E_{x}=\{y\}$, $\operatorname{logo} \nu(E)=\sum_{x \in C_{1}} \mu_{x}(E)=0$. Assim, para qualquer $f \in L^{1}\left(E, \mathcal{A}^{\prime}, \mu^{\prime}\right)$ 
segue que $\alpha(f)=\int_{X} f \mathrm{~d} \nu=0$, ou seja, $\alpha$ se anula em $L^{1}\left(E, \mathcal{A}^{\prime}, \mu^{\prime}\right)$. Portanto, a injetividade da aplicação de Riesz do espaço $\left(E, \mathcal{A}^{\prime}, \mu^{\prime}\right)$ implica que $\left.g\right|_{E}=0 \mu$-qs. Seja $R=g^{-1}(1)$. Uma vez que $g$ é $\mathcal{A}$-mensurável e $\{1\} \in \mathcal{B}(\mathbb{R})$ temos $R \in \mathcal{A}$. Se $S$ é o conjunto $R^{y} \times\{y\}$ então

$$
\mu(S) \leq \mu(\{x \in E: g(x) \neq 0\})=0 \text {, i.e., } \mu(S)=0 .
$$

Logo $\mu^{y}(S)=0$, e portanto $R^{y}$ é enumerável. Seja agora $x \in C_{1}$ fixado e consideremos o conjunto $F=\{x\} \times C_{2}$. Uma vez que $F^{y}=\{x\}$ para cada $y \in C_{2}$, temos $\sum_{y \in C_{2}} \mu^{y}(F)=0$, o que implica que $\mu$ e $\nu$ coincidem em todos os subconjuntos mensuráveis de $F$. Portanto, se $\mathcal{A}^{\prime \prime}=\left.\mathcal{A}\right|_{F}$ e $\mu^{\prime \prime}=\left.\mu\right|_{\mathcal{A}^{\prime \prime}}$ então $\alpha(f)=\int_{X} f \mathrm{~d} \mu$ para cada $f \in L^{1}\left(F, \mathcal{A}^{\prime \prime}, \mu^{\prime \prime}\right)$. Novamente a aplicação de Riesz

$$
L^{\infty}\left(F, \mathcal{A}^{\prime \prime}, \mu^{\prime \prime}\right) \stackrel{\beta}{\longrightarrow} L^{1}\left(F, \mathcal{A}^{\prime \prime}, \mu^{\prime \prime}\right)^{*}
$$

é injetora e $\left.g\right|_{F}$ é levado na restrição de $\alpha$ a $L^{1}\left(F, \mathcal{A}^{\prime \prime}, \mu^{\prime \prime}\right)$. Assim, para cada $f \in L^{1}\left(F, \mathcal{A}^{\prime \prime}, \mu^{\prime \prime}\right)$, temos

$$
\beta_{\left.g\right|_{F}}(f)=\alpha(f)=\int_{X} f \mathrm{~d} \mu=\int_{F} f \cdot 1 \mathrm{~d} \mu^{\prime \prime}=\beta_{1}(f),
$$

i.e., $\beta_{\left.g\right|_{F}}(f)=\beta_{1}(f)$. Como $\beta$ é injetora segue que $\left.g\right|_{F}=1 \mu^{\prime \prime}$-qs, que é o mesmo que $\left.g\right|_{F}=1 \mu$-qs. Seja $K=\{x\} \times R_{x} \subset F$. Então

$$
\mu(F \backslash K) \leq \mu(\{x \in E: g(x) \neq 1\})=0,
$$

ou seja, $(F \backslash K)_{x}$ é enumerável. Mas $(F \backslash K)_{x}=C_{2} \backslash K_{x}=C_{2} \backslash R_{x}$. Assim, $R$ é um conjunto mensurável de $X$ tal que $R^{y}$ e $C_{2} \backslash R_{x}$ são enumeráveis para todo $x \in C_{1}$ e $y \in C_{2}$.

Proposição 2.4.4. Se $C_{1}$ e $C_{2}$ são conjuntos não enumeráveis então as seguintes condições são equivalentes:

- $\left|C_{1}\right|=\left|C_{2}\right|=\aleph_{1}$;

- existe um subconjunto $R$ de $C_{1} \times C_{2}$ tal que $R^{y}$ e $C_{2} \backslash R_{x}$ são enumeráveis para todo $x \in C_{1}$ e $y \in C_{2}$.

DemonstraÇão. Se $\left|C_{1}\right|=\left|C_{2}\right|=\aleph_{1}$ então nós podemos assumir que $C_{1}=C_{2}=\aleph_{1}$. Seja $R=\left\{(x, y) \in \aleph_{1} \times \aleph_{1}: x \in y\right\}$. Para cada $y \in C_{2}$ temos

$$
R^{y}=\left\{x \in \aleph_{1}:(x, y) \in R\right\}=\left\{x \in \aleph_{1}: x<y\right\}=y \in \aleph_{1},
$$


e para cada $x \in C_{1}$ temos

$$
\begin{aligned}
C_{2} \backslash R_{x}=\left\{y \in \aleph_{1}:\right. & (x, y) \notin R\}=\left\{y \in \aleph_{1}: y \leq x\right\} \\
& =\left\{y \in \aleph_{1}: y<x\right\} \cup\{x\}=x \cup\{x\}=x+1 \in \aleph_{1} .
\end{aligned}
$$

Como todo elemento de $\aleph_{1}$ é um número ordinal enumerável, segue que $R^{y}$ e $C_{2} \backslash R_{x}$ são enumeráveis para todo $x \in C_{1}$ e $y \in C_{2}$. Reciprocamente, suponhamos que exista $R \subset C_{1} \times C_{2}$ tal que $R^{y}$ e $C_{2} \backslash R_{x}$ sejam enumeráveis para todo $x \in C_{1}$ e $y \in C_{2}$. Uma vez que $C_{1}$ é não enumerável, existe um subconjunto $A \subset C_{1}$ tal que $|A|=\aleph_{1}$. Afirmamos que $\bigcap_{x \in A} R_{x}=\emptyset$. De fato, se existisse $y \in \bigcap_{x \in A} R_{x}$, teríamos $y \in R_{x}$ para todo $x \in A$, e portanto $A \subset R^{y}$, o que contradiz a hipótese de enumerabilidade de $R^{y}$. Então

$$
\begin{aligned}
C_{2}=C_{2} \backslash \bigcap_{x \in A} R_{x}=C_{2} \cap\left(\bigcap_{x \in A} R_{x}\right)^{c}= & C_{2} \cap\left(\bigcup_{x \in A} R_{x}^{c}\right) \\
& =\bigcup_{x \in A}\left(C_{2} \cap R_{x}^{c}\right)=\bigcup_{x \in A}\left(C_{2} \backslash R_{x}\right) .
\end{aligned}
$$

Mas $C_{2} \backslash R_{x}$ é enumerável para todo $x \in C_{1}$ e $A$ tem cardinalidade igual a $\aleph_{1}$, logo $\left|C_{2}\right| \leq \aleph_{1} \cdot \aleph_{0}=\aleph_{1}$, provando que $\left|C_{2}\right|=\aleph_{1}$. Observe agora que $C_{1}=\bigcup_{y \in C_{2}} R^{y}$; com efeito, para $x \in C_{1}$ o conjunto $C_{2} \backslash R_{x}$ é enumerável, logo existe $y \in R_{x}$, e conseqüentemente $x \in R^{y}$. Assim, $C_{1} \subset \bigcup_{y \in C_{2}} R^{y}$, e é claro que $\bigcup_{y \in C_{2}} R^{y} \subset C_{1}$. Como $R^{y}$ é enumerável para todo $y \in C_{2}$, temos $\left|C_{1}\right| \leq \aleph_{1} \cdot \aleph_{0}=\aleph_{1}$ e portanto $\left|C_{1}\right|=\aleph_{1}$.

Corolário 2.4.5. Se $C_{1}$ e $C_{2}$ são não enumeráveis e $C_{1}$ ou $C_{2}$ tem cardinalidade maior do que $\aleph_{1}$ então a aplicação de Riesz (1.4.4) do espaço $(X, \mathcal{A}, \mu)$ do Exemplo 2.4.1 não é um isomorfismo.

DemonstraçÃo. Segue diretamente do Lema 2.4.3 e da Proposição 2.4.4.

O Lema 2.4.3 e a Proposição 2.4.4 nos dizem que para o espaço de medida construído no Exemplo 2.4.1 se a aplicação de Riesz (1.4.4) desse espaço é um isomorfismo então os conjuntos $C_{1}$ e $C_{2}$ têm ambos cardinalidade igual a $\aleph_{1}$. Veremos no capítulo 4 (Exemplo 4.1 .17 e Corolário 4.2.9) que a recíproca também é verdadeira, i.e., se $\left|C_{1}\right|=\left|C_{2}\right|=\aleph_{1}$ então a aplicação de Riesz (1.4.4) é um isomorfismo. 


\section{CAPíTULO 3}

\section{Somas}

\subsection{Soma externa}

Dada uma família de conjuntos $\left(X_{i}\right)_{i \in I}$, a sua união disjunta, denotada por $\sum_{i \in I} X_{i}$, é definida por

$$
\sum_{i \in I} X_{i}=\bigcup_{i \in I}\left(\{i\} \times X_{i}\right) .
$$

Para simplificar a notação, exceto em situações em que se possa causar confusão, nós identificamos cada $x \in X_{i} \operatorname{com}(i, x)$, e portanto $X_{i}$ pode ser visto (a menos de identificação) como um subconjunto de $\sum_{i \in I} X_{i}$.

DEFInIÇÃo 3.1.1. A soma externa de uma família de espaços de medida $\left(X_{i}, \mathcal{A}_{i}, \mu_{i}\right)_{i \in I}$, denotada por $\sum_{i \in I}\left(X_{i}, \mathcal{A}_{i}, \mu_{i}\right)$, é um espaço de medida $(X, \mathcal{A}, \mu)$ assim definido:

- $X=\sum_{i \in I} X_{i}$;

- $\mathcal{A}=\left\{A \subset X: A \cap X_{i} \in \mathcal{A}_{i}\right.$, para todo $\left.i \in I\right\}$;

- $\mu(A)=\sum_{i \in I} \mu_{i}\left(A \cap X_{i}\right)$, para todo $A \in \mathcal{A}$.

Observamos que a soma externa $(X, \mathcal{A}, \mu)=\sum_{i \in I}\left(X_{i}, \mathcal{A}_{i}, \mu_{i}\right)$ está bem definida: se para cada $i \in I, f_{i}: X_{i} \rightarrow X$ é a aplicação inclusão, então $\mathcal{A}$ é a $\sigma$-álgebra co-induzida em $X$ pela família de funções $\left(f_{i}\right)_{i \in I}$. Além disso, $\mu(\emptyset)=\sum_{i \in I} \mu_{i}\left(\emptyset \cap X_{i}\right)=0$ e se $\left(A_{j}\right)_{j \geq 1}$ é uma seqüência de elementos de $\mathcal{A}$, dois a dois disjuntos, então:

$$
\begin{aligned}
\mu\left(\bigcup_{j \geq 1} A_{j}\right)= & \sum_{i \in I} \mu_{i}\left(\left(\bigcup_{j \geq 1} A_{j}\right) \cap X_{i}\right)=\sum_{i \in I} \mu_{i}\left(\bigcup_{j \geq 1}\left(A_{j} \cap X_{i}\right)\right) \\
& =\sum_{i \in I} \sum_{j \geq 1} \mu_{i}\left(A_{j} \cap X_{i}\right)=\sum_{j \geq 1} \sum_{i \in I} \mu_{i}\left(A_{j} \cap X_{i}\right)=\sum_{j \geq 1} \mu\left(A_{j}\right) .
\end{aligned}
$$

Portanto a aplicação $\mu$ é uma medida em $X$. Observamos ainda que, fixado $i \in I$, se $A \subset X_{i}$ então $A \in \mathcal{A}$ se e somente se $A \in \mathcal{A}_{i}$. Com efeito, se $A \in \mathcal{A}$ então $A=A \cap X_{i} \in \mathcal{A}_{i}$; reciprocamente, se $A \in \mathcal{A}_{i}$ então $A \cap X_{i}=A$ e 
$A \cap X_{j}=\emptyset$ para $j \neq i$, e portanto $A \in \mathcal{A}$. Decorre daí que $\mu(A)=\mu_{i}(A)$ e que $\mathcal{A}_{i} \subset \mathcal{A}$ (portanto $X_{i} \in \mathcal{A}$ ).

ObservaÇão 3.1.2. Se $\left(X_{i}, \mathcal{A}_{i}, \mu_{i}\right)_{i \in I}$ é uma família de espaços de medida e $(X, \mathcal{A}, \mu)=\sum_{i \in I}\left(X_{i}, \mathcal{A}_{i}, \mu_{i}\right)$ é a sua soma externa então, pela Proposição 1.4.35, uma aplicação $f$ definida em $X$ é mensurável se e somente se $\left.f\right|_{X_{i}}$ é mensurável para todo $i \in I$.

Proposição 3.1.3. Se $\left(X_{i}, \mathcal{A}_{i}, \mu_{i}\right)_{i \in I}$ é uma família de espaços de medida e $(X, \mathcal{A}, \mu)=\sum_{i \in I}\left(X_{i}, \mathcal{A}_{i}, \mu_{i}\right)$ é a sua soma externa, então:

(a) se cada $\mu_{i}$ é completa então $\mu$ é completa;

(b) se cada $\mu_{i}$ é cheia então $\mu$ é cheia;

(c) se cada $\mu_{i}$ é livre de blocos então $\mu$ é livre de blocos;

(d) se cada $\mu_{i}$ é perfeita então $\mu$ é perfeita.

\section{DEMONSTRAÇÃO.}

- Prova de (a).

Sejam $A \in \mathcal{A} \operatorname{com} \mu(A)=0$ e $B$ um subconjunto de $A$. Se $A \in \mathcal{A}$ então $A \cap X_{i} \in \mathcal{A}_{i}$ para todo $i \in I$. Portanto, para cada $i \in I$, temos $A \cap X_{i} \in \mathcal{A}$ e $\mu_{i}\left(A \cap X_{i}\right)=\mu\left(A \cap X_{i}\right) \leq \mu(A)=0$, i.e., $\mu_{i}\left(A \cap X_{i}\right)=0$. Como $B \cap X_{i} \subset A \cap X_{i}$ e $\mu_{i}$ é completa, segue que $B \cap X_{i} \in \mathcal{A}_{i}$ para todo $i \in I$; portanto $B \in \mathcal{A}$, e isso mostra que $\mu$ é uma medida completa.

- Prova de (b).

Seja $A \subset X$ e suponhamos que para todo $B \in \mathcal{A} \operatorname{com} \mu(B)<+\infty$ o conjunto $A \cap B$ está em $\mathcal{A}$. Fixemos $i \in I$. Se $E \in \mathcal{A}_{i}$ e $\mu_{i}(E)<+\infty$ então $E \in \mathcal{A}$ e $\mu(E)=\mu_{i}(E)<+\infty$; $\operatorname{logo} A \cap E \in \mathcal{A}$, e portanto $\left(A \cap X_{i}\right) \cap E=(A \cap E) \cap X_{i} \in \mathcal{A}_{i}$. Como $\mu_{i}$ é cheia temos que $A \cap X_{i} \in \mathcal{A}_{i}$ para todo $i \in I$, e assim $A \in \mathcal{A}$. Portanto $\mu$ é uma medida cheia.

- Prova de (c).

Seja $A \in \mathcal{A} \operatorname{com} \mu(A)=+\infty$. Como $\mu(A)=\sum_{i \in I} \mu_{i}\left(A \cap X_{i}\right)$ temos que $\mu_{i}\left(A \cap X_{i}\right)>0$ para algum $i \in I$. Se $\mu_{i}\left(A \cap X_{i}\right)<+\infty$ então $0<\mu\left(A \cap X_{i}\right)<+\infty$, e portanto $A$ não é um bloco infinito para $\mu$. Se $\mu_{i}\left(A \cap X_{i}\right)=+\infty$ então, uma vez que $\mu_{i}$ é livre de blocos, existe $E \in \mathcal{A}_{i}$ contido em $A \cap X_{i}$ com $0<\mu_{i}(E)=\mu(E)<+\infty$, provando novamente que $A$ não é um bloco infinito para $\mu$. 
- Prova de (d).

Segue de (a), (b) e (c).

Lema 3.1.4. Sejam $\left(X_{i}, \mathcal{A}_{i}, \mu_{i}\right)_{i \in I}$ uma família de espaços de medida e $(X, \mathcal{A}, \mu)=\sum_{i \in I}\left(X_{i}, \mathcal{A}_{i}, \mu_{i}\right)$ a sua soma externa. Se $\varphi: X \rightarrow \mathbb{R}$ é uma função simples, mensurável e não negativa então:

$$
\int_{X} \varphi \mathrm{d} \mu=\sum_{i \in I} \int_{X_{i}} \varphi \mathrm{d} \mu
$$

Demonstração. Para cada $i \in I$ temos:

$$
\varphi \chi_{X_{i}}=\left(\sum_{c \in \operatorname{Im} \varphi} c \chi_{\varphi^{-1}(c)}\right) \chi_{X_{i}}=\sum_{c \in \operatorname{Im} \varphi}\left(c \chi_{\varphi^{-1}(c)} \chi_{X_{i}}\right)=\sum_{c \in \operatorname{Im} \varphi} c \chi_{\varphi^{-1}(c) \cap X_{i}} .
$$

Portanto, para todo $i \in I$,

$$
\int_{X_{i}} \varphi \mathrm{d} \mu=\int_{X} \varphi \chi_{X_{i}} \mathrm{~d} \mu=\sum_{c \in \operatorname{Im} \varphi} c \mu\left(\varphi^{-1}(c) \cap X_{i}\right) .
$$

Assim,

$$
\begin{aligned}
\int_{X} \varphi \mathrm{d} \mu=\sum_{c \in \operatorname{Im} \varphi} c \mu\left(\varphi^{-1}(c)\right) & =\sum_{c \in \operatorname{Im} \varphi}\left(c \sum_{i \in I} \mu_{i}\left(\varphi^{-1}(c) \cap X_{i}\right)\right) \\
& =\sum_{i \in I} \sum_{c \in \operatorname{Im} \varphi} c \mu\left(\varphi^{-1}(c) \cap X_{i}\right)=\sum_{i \in I} \int_{X_{i}} \varphi \mathrm{d} \mu .
\end{aligned}
$$

Proposição 3.1.5. Sejam $\left(X_{i}, \mathcal{A}_{i}, \mu_{i}\right)_{i \in I}$ uma família de espaços de medida e $(X, \mathcal{A}, \mu)=\sum_{i \in I}\left(X_{i}, \mathcal{A}_{i}, \mu_{i}\right)$ a sua soma externa. Se $f: X \rightarrow \mathbb{R} e ́$ uma função mensurável e não negativa então:

$$
\int_{X} f \mathrm{~d} \mu=\sum_{i \in I} \int_{X_{i}} f \mathrm{~d} \mu .
$$

Demonstração. Para cada $F \subset I$ finito temos que

$$
\sum_{i \in F} \int_{X_{i}} f \mathrm{~d} \mu=\int_{\sum_{i \in F} X_{i}} f \mathrm{~d} \mu \leq \int_{\sum_{i \in I} X_{i}} f \mathrm{~d} \mu=\int_{X} f \mathrm{~d} \mu .
$$

Portanto,

$$
\sum_{i \in I} \int_{X_{i}} f \mathrm{~d} \mu=\sup _{\substack{F \subset I \\ \text { F finito }}}\left(\sum_{i \in F} \int_{X_{i}} f \mathrm{~d} \mu\right) \leq \int_{X} f \mathrm{~d} \mu
$$


Por outro lado, pelo Lema 3.1.4, para cada função $\varphi: X \rightarrow \mathbb{R}$ simples, mensurável e não negativa $\operatorname{com} \varphi \leq f$ temos:

$$
\int_{X} \varphi \mathrm{d} \mu=\sum_{i \in I} \int_{X_{i}} \varphi \mathrm{d} \mu \leq \sum_{i \in I} \int_{X_{i}} f \mathrm{~d} \mu .
$$

Assim, $\int_{X} f \mathrm{~d} \mu \leq \sum_{i \in I} \int_{X_{i}} f \mathrm{~d} \mu$, uma vez que

$$
\int_{X} f \mathrm{~d} \mu=\sup \left\{\int_{X} \varphi \mathrm{d} \mu \mid \varphi: X \rightarrow \mathbb{R} \text { simples, mensurável e } 0 \leq \varphi \leq f\right\} \text {. }
$$

\subsection{Norma e Soma direta de tipo $L^{p}$}

DefiniçÃo 3.2.1. Seja $\left(E_{i}\right)_{i \in I}$ uma família de espaços de Banach. Para $v=\left(v_{i}\right)_{i \in I} \in \prod_{i \in I} E_{i}$ e $p \in[1,+\infty]$ a norma de tipo- $L^{p}$ de $v$ é definida por:

$$
\|v\|_{p}=\left(\sum_{i \in I}\left\|v_{i}\right\|^{p}\right)^{\frac{1}{p}}, \quad \text { se } p<+\infty, \quad \text { e } \quad\|v\|_{\infty}=\sup _{i \in I}\left\|v_{i}\right\| .
$$

A soma direta (externa) de tipo- $L^{p}$ da família $\left(E_{i}\right)_{i \in I}$ é definida por:

$$
\bar{\bigoplus}_{i \in I}^{p} E_{i}=\left\{v \in \prod_{i \in I} E_{i}:\|v\|_{p}<+\infty\right\} .
$$

Lema 3.2.2. Seja $\left(E_{i}\right)_{i \in I}$ uma família de espaços de Banach. Para cada $p \in[1,+\infty]$, quaisquer que sejam $u=\left(u_{i}\right)_{i \in I}, v=\left(v_{i}\right)_{i \in I} \in \prod_{i \in I} E_{i}$ temos

$$
\|u+v\|_{p} \leq\|u\|_{p}+\|v\|_{p}
$$

DemonstraÇÃo. Vejamos primeiramente o caso $p=+\infty$. Para todo $i \in I$ temos:

$$
\left\|u_{i}\right\|+\left\|v_{i}\right\| \leq \sup _{j \in I}\left\|u_{j}\right\|+\sup _{j \in I}\left\|v_{j}\right\|
$$

Portanto,

$$
\begin{aligned}
\|u+v\|_{\infty}=\sup _{i \in I}\left\|u_{i}+v_{i}\right\| & \leq \sup _{i \in I}\left(\left\|u_{i}\right\|+\left\|v_{i}\right\|\right) \\
& \stackrel{(*)}{\leq} \sup _{j \in I}\left\|u_{j}\right\|+\sup _{j \in I}\left\|v_{j}\right\|=\|u\|_{\infty}+\|v\|_{\infty} .
\end{aligned}
$$

Seja agora $p<+\infty$ e consideremos o espaço $L^{p}(I, \mathcal{P}(I), \mu)$, no qual $\mu$ é a medida de contagem. Pela Observação 1.4.78 temos

$$
\|u\|_{p}=\left(\sum_{i \in I}\left\|u_{i}\right\|^{p}\right)^{\frac{1}{p}}=\left\|\left(\left\|u_{i}\right\|\right)_{i \in I}\right\|_{p}
$$


em que $\|u\|_{p}$ é a norma de tipo- $L^{p}$ de $u$ em $\prod_{i \in I} E_{i}$, e $\left\|\left(\left\|u_{i}\right\|\right)_{i \in I}\right\|_{p}$ é a norma da família de números reais $\left(\left\|u_{i}\right\|\right)_{i \in I} \operatorname{em~} L^{p}(I, \mathcal{P}(I), \mu)$. Analogamente,

$$
\|v\|_{p}=\left\|\left(\left\|v_{i}\right\|\right)_{i \in I}\right\|_{p} \quad \text { e }\|u+v\|_{p}=\left\|\left(\left\|u_{i}+v_{i}\right\|\right)_{i \in I}\right\|_{p} .
$$

Como $\left\|u_{i}+v_{i}\right\| \leq\left\|u_{i}\right\|+\left\|v_{i}\right\|$ para cada $i \in I$, pela Observação 1.4.78, temos:

$$
\left\|\left(\left\|u_{i}+v_{i}\right\|\right)_{i \in I}\right\|_{p} \leq\left\|\left(\left\|u_{i}\right\|+\left\|v_{i}\right\|\right)_{i \in I}\right\|_{p} .
$$

Assim, desse fato e da desigualdade de Minkowski segue que

$$
\begin{aligned}
\|u+v\|_{p}= & \left\|\left(\left\|u_{i}+v_{i}\right\|\right)_{i \in I}\right\|_{p} \leq\left\|\left(\left\|u_{i}\right\|+\left\|v_{i}\right\|\right)_{i \in I}\right\|_{p} \\
& =\left\|\left(\left\|u_{i}\right\|\right)_{i \in I}+\left(\left\|v_{i}\right\|\right)_{i \in I}\right\|_{p} \leq\left\|\left(\left\|u_{i}\right\|\right)_{i \in I}\right\|_{p}+\left\|\left(\left\|v_{i}\right\|\right)_{i \in I}\right\|_{p},
\end{aligned}
$$

ou seja, $\|u+v\|_{p} \leq\|u\|_{p}+\|v\|_{p}$.

Corolário 3.2.3. Se $\left(E_{i}\right)_{i \in I}$ é uma família de espaços de Banach então, para cada $p \in[1,+\infty], \bar{\bigoplus}_{i \in I}^{p} E_{i}$ é um subespaço de $\prod_{i \in I} E_{i}$ e $\|\cdot\|_{p}$ é uma norma em $\bar{\bigoplus}_{i \in I}^{p} E_{i}$.

Demonstração. Seja $p \in[1,+\infty]$. A família nula em $\prod_{\in I} E_{i}$ tem norma tipo- $L^{p}$ igual a zero e portanto está em $\bar{\bigoplus}_{i \in I}^{p} E_{i}$. Além disso, para todo $u, v \in \bar{\bigoplus}_{i \in I}^{p} E_{i}$ e $\alpha \in \mathbb{R}$ temos trivialmente que $\|\alpha u\|_{p}=|\alpha|\|u\|_{p}<+\infty$ e, pelo Lema 3.2.2, $\|u+v\|_{p} \leq\|u\|_{p}+\|v\|_{p}<+\infty$. Portanto a soma direta de tipo- $L^{p}$ da família $\left(E_{i}\right)_{i \in I}$ é um subespaço vetorial de $\prod_{\in I} E_{i}$. Observe que para todo $u \in \bar{\bigoplus}_{i \in I}^{p} E_{i}$ temos $0 \leq\|u\|_{p}<+\infty$, e se $\|u\|_{p}=0$ então $u=0$. De fato, se $\|u\|_{\infty}=0$ então $\sup _{i \in I}\left\|u_{i}\right\|=0$, e portanto $\left\|u_{i}\right\| \leq 0$ para todo $i \in I$, o que implica $u_{i}=0$ para cada $i \in I$. Se $p<+\infty$ e $\|u\|_{p}=0$ então $\sum_{i \in I}\left\|u_{i}\right\|^{p}=0$, e portanto $\left\|u_{i}\right\|=0$ para todo $i \in I$, ou seja, $u_{i}=0$ para cada $i \in I$. Isso mostra que $\|\cdot\|_{p}$ é uma norma em $\bar{\bigoplus}_{i \in I}^{p} E_{i}$.

Proposição 3.2.4. Se $\left(E_{i}\right)_{i \in I}$ é uma familia de espaços de Banach então, para cada $p \in[1,+\infty]$, o par $\left(\bar{\bigoplus}_{i \in I}^{p} E_{i},\|\cdot\|_{p}\right)$ é um espaço de Banach.

DemonstraçÃo. Faremos a demonstração da proposição dividindo-a nos casos $p=+\infty$ e $p<+\infty$. Seja $\left(x_{n}\right)_{n \geq 1}$ uma seqüência de Cauchy em $\bar{\bigoplus}_{i \in I}^{\infty} E_{i}$, na qual $x_{n}=\left(x_{n}^{i}\right)_{i \in I}$ para cada $n \geq 1$. Dado $\varepsilon>0$, existe um número natural $k \geq 1$ tal que

$$
\left\|x_{n}-x_{m}\right\|_{\infty}=\sup _{i \in I}\left\|x_{n}^{i}-x_{m}^{i}\right\|<\varepsilon
$$


para todo $n, m>k$. Logo, para cada $i \in I$, temos $\left\|x_{n}^{i}-x_{m}^{i}\right\|<\varepsilon$ para todo $n, m>k$. Portanto $\left(x_{n}^{i}\right)_{n \geq 1}$ é uma seqüência de Cauchy em $E_{i}$, e uma vez que $E_{i}$ é um espaço de Banach, existe $x^{i} \in E_{i}$ tal que $x_{n}^{i} \longrightarrow x^{i}$. Seja $x=\left(x^{i}\right)_{i \in I}$. Vamos mostrar que $x \in \bar{\bigoplus}_{i \in I}^{\infty} E_{i}$ e que $x_{n} \longrightarrow x$ na norma $\|\cdot\|_{\infty}$, ou seja, $\left\|x_{n}-x\right\|_{\infty} \longrightarrow 0$. Se $\left\|x_{n}^{i}-x_{m}^{i}\right\|<\varepsilon$ para cada $i \in I$ e para todo $n, m>k$ então, fazendo $m \longrightarrow+\infty$, temos $\left\|x_{n}^{i}-x^{i}\right\| \leq \varepsilon$ para cada $i \in I$ e $n>k$. Por outro lado, $x_{n}=\left(x_{n}^{i}\right)_{i \in I} \in \bar{\bigoplus}_{i \in I}^{\infty} E_{i}$, logo $\sup _{i \in I}\left\|x_{n}^{i}\right\| \stackrel{\text { def }}{=} k_{n}<+\infty$, ou seja, $\left\|x_{n}^{i}\right\| \leq k_{n}$ para todo $i \in I$. Portanto

$$
\left\|x^{i}\right\| \leq\left\|x^{i}-x_{n}^{i}\right\|+\left\|x_{n}^{i}\right\| \leq \varepsilon+k_{n} \text { para todo } i \in I,
$$

e daí $\|x\|_{\infty}=\sup _{i \in I}\left\|x^{i}\right\| \leq \varepsilon+k_{n}<+\infty$, i.e., $x \in \bar{\bigoplus}_{i \in I}^{\infty} E_{i}$. Ainda, como

$$
\left\|x_{n}-x\right\|_{\infty}=\sup _{i \in I}\left\|x_{n}^{i}-x^{i}\right\| \leq \varepsilon \text { para todo } n>k \text { e todo } \varepsilon>0,
$$

segue que $\left\|x_{n}-x\right\|_{\infty} \longrightarrow x$. Portanto $\left(\bar{\bigoplus}_{i \in I}^{\infty} E_{i},\|\cdot\|_{\infty}\right)$ é um espaço normado completo. Sejam agora $p \in\left[1,+\infty\left[\right.\right.$ e $\left(x_{n}\right)_{n \geq 1}$ uma seqüência de Cauchy em $\bar{\bigoplus}_{i \in I}^{p} E_{i}$, em que $x_{n}=\left(x_{n}^{i}\right)_{i \in I}$ para cada $n \geq 1$. Dado $\varepsilon>0$, existe um número natural $k \geq 1$ tal que

$$
\left\|x_{n}-x_{m}\right\|_{p}=\left(\sum_{i \in I}\left\|x_{n}^{i}-x_{m}^{i}\right\|^{p}\right)^{\frac{1}{p}}<\varepsilon,
$$

para todo $n, m>k$. Logo, para cada $i \in I$, temos $\left\|x_{n}^{i}-x_{m}^{i}\right\|<\varepsilon$ para todo $n, m>k$, ou seja, $\left(x_{n}^{i}\right)_{n \geq 1}$ é uma seqüência de Cauchy em $E_{i}$, e portanto existe $x^{i} \in E_{i}$ tal que $x_{n}^{i} \longrightarrow x^{i}$. Seja $x=\left(x^{i}\right)_{i \in I}$. Mostraremos que $x \in \bar{\bigoplus}_{i \in I}^{p} E_{i}$ e que $x_{n} \longrightarrow x$ na norma $\|\cdot\|_{p}$, ou seja, $\left\|x_{n}-x\right\|_{p} \longrightarrow 0$. Para todo $n, m>k$ temos $\sum_{i \in I^{\prime}}\left\|x_{n}^{i}-x_{m}^{i}\right\|^{p} \leq \varepsilon^{p}$, para cada $I^{\prime} \subset I$ finito. Portanto, fazendo $m \longrightarrow+\infty$ concluímos que $\sum_{i \in I^{\prime}}\left\|x_{n}^{i}-x^{i}\right\|^{p} \leq \varepsilon^{p}$ para cada $I^{\prime} \subset I, I^{\prime}$ finito. Assim,

$$
\sum_{i \in I}\left\|x_{n}^{i}-x^{i}\right\|^{p}=\sup _{\substack{I^{\prime} \subset I \\ I^{\prime} \text { finito }}} \sum_{i \in I^{\prime}}\left\|x_{n}^{i}-x^{i}\right\|^{p} \leq \varepsilon^{p},
$$

e isso mostra que $x_{n}-x \in \bar{\bigoplus}_{i \in I}^{p} E_{i}$. Como $x_{n} \in \bar{\bigoplus}_{i \in I}^{p} E_{i}$ e $\bar{\bigoplus}_{i \in I}^{p} E_{i}$ é um subespaço vetorial de $\prod_{i \in I} E_{i}$, segue que $x=\left(x-x_{n}\right)+x_{n} \in \bar{\bigoplus}_{i \in I}^{p} E_{i}$. Além disso, uma vez que

$$
\left\|x_{n}-x\right\|_{p}=\left(\sum_{i \in I}\left\|x_{n}^{i}-x^{i}\right\|^{p}\right)^{\frac{1}{p}} \leq \varepsilon,
$$


para todo $n>k$ e todo $\varepsilon>0$, temos $\left\|x_{n}-x\right\|_{p} \longrightarrow 0$. Portanto, para cada $p \in\left[1,+\infty\left[\right.\right.$, o par $\left(\bar{\bigoplus}_{i \in I}^{p} E_{i},\|\cdot\|_{p}\right)$ é um espaço de Banach.

OBSERVAÇÃo 3.2.5. Se $p<+\infty$ então para $v=\left(v_{i}\right)_{i \in I} \in \bar{\bigoplus}_{i \in I}^{p} E_{i}$ temos $v_{i}=0$ exceto para uma quantidade enumerável de índices $i \in I$. Com efeito, uma vez que $\sum_{i \in I}\left\|v_{i}\right\|^{p}<+\infty$, de acordo com a Observação 1.3.15, o conjunto dos índices $i \in I$ tais que $v_{i} \neq 0$ é enumerável.

ObservaÇÃo 3.2.6. Se $\left(E_{i}\right)_{i \in I}$ é uma família de espaços de Banach, $p \in[1,+\infty]$ e $\alpha \in\left(\bar{\bigoplus}_{i \in I}^{p} E_{i}\right)^{*}$, então $\left(\left.\alpha\right|_{E_{i}}\right)_{i \in I} \in \prod_{i \in I} E_{i}^{*}$. De fato, fixado $i \in I$, para todo $u \in E_{i}$ temos

$$
\left|\alpha_{i}(u)\right|=|\alpha(u)| \leq\|\alpha\|\|u\|_{p}=\|\alpha\|\|u\|, \quad \text { i.e., } \quad \alpha_{i} \in E_{i}^{*} .
$$

Portanto $\left(\left.\alpha\right|_{E_{i}}\right)_{i \in I}$ é uma família em $\prod_{i \in I} E_{i}^{*}$. Note que, para cada $i \in I$, estamos identificando $E_{i}$ com o subespaço $\left\{\left(\delta_{i j} v_{i}\right)_{j \in I} \in \prod_{j \in I} E_{j}: v_{i} \in E_{i}\right\}^{1}$. Assim, se $u_{i} \in E_{i}$ então $\left\|u_{i}\right\|=\left\|\left(\delta_{i j} u_{i}\right)_{j \in I}\right\|_{p}=\left\|u_{i}\right\|_{p}$.

Lema 3.2.7. Seja $\left(E_{i}\right)_{i \in I}$ uma família de espaços de Banach. Dados $p, q \in[1,+\infty]$ com $\frac{1}{p}+\frac{1}{q}=1$, para cada família $\left(\alpha_{i}\right)_{i \in I} \in \prod_{i \in I} E_{i}^{*}$, se $\left(x_{i}\right)_{i \in I} \in \prod_{i \in I} E_{i}$ então:

$$
\sum_{i \in I}\left|\alpha_{i}\left(x_{i}\right)\right| \leq\left\|\left(\alpha_{i}\right)_{i \in I}\right\|_{q}\left\|\left(x_{i}\right)_{i \in I}\right\|_{p}
$$

DemonstraÇÃo. Sejam $\left(\alpha_{i}\right)_{i \in I} \in \prod_{i \in I} E_{i}^{*}$ e $\left(x_{i}\right)_{i \in I} \in \prod_{i \in I} E_{i}$. Como $\left|\alpha_{i}\left(x_{i}\right)\right| \leq\left\|\alpha_{i}\right\|\left\|x_{i}\right\|$ para todo $i \in I$, temos $\sum_{i \in I}\left|\alpha_{i}\left(x_{i}\right)\right| \leq \sum_{i \in I}\left\|\alpha_{i}\right\|\left\|x_{i}\right\|$. Assim, pela desigualdade de Hölder, segue que

$$
\sum_{i \in I}\left|\alpha_{i}\left(x_{i}\right)\right| \leq\left\|\left(\left\|\alpha_{i}\right\|\right)_{i \in I}\right\|_{q}\left\|\left(\left\|x_{i}\right\|\right)_{i \in I}\right\|_{p}=\left\|\left(\alpha_{i}\right)_{i \in I}\right\|_{q}\left\|\left(x_{i}\right)_{i \in I}\right\|_{p} .
$$

Proposição 3.2.8. Seja $\left(E_{i}\right)_{i \in I}$ uma familia de espaços de Banach. Dados $p, q \in[1,+\infty]$ com $\frac{1}{p}+\frac{1}{q}=1$, temos que para cada funcional linear limitado a sobre $\bar{\bigoplus}_{i \in I}^{p} E_{i}$, a família $\left(\left.\alpha\right|_{E_{i}}\right)_{i \in I}$ está em $\bar{\bigoplus}_{i \in I}^{q} E_{i}^{*}$. Além disso, se $p<+\infty$ então $\alpha(v)=\sum_{i \in I} \alpha\left(v_{i}\right)$ para todo $v=\left(v_{i}\right)_{i \in I} \in \bar{\bigoplus}_{i \in I}^{p} E_{i}$ e é uma isometria linear a aplicação

$$
\left(\bar{\bigoplus}_{i \in I}^{p} E_{i}\right)^{*} \ni \alpha \longmapsto\left(\left.\alpha\right|_{E_{i}}\right)_{i \in I} \in \bar{\bigoplus}_{i \in I}^{q} E_{i}^{*}
$$

\footnotetext{
${ }^{1} \delta_{i j}=1$ se $i=j$ e $\delta_{i j}=0$ se $i \neq j$.
} 
Demonstração. Vamos mostrar inicialmente que se $\alpha: \bar{\bigoplus}_{i \in I}^{p} E_{i} \rightarrow \mathbb{R}$ é um funcional linear limitado então $\left(\left.\alpha\right|_{E_{i}}\right)_{i \in I} \in \bar{\bigoplus}_{i \in I}^{q} E_{i}^{*}$. Faremos isso dividindo a prova em três casos:

- $p=1$ e $q=+\infty$.

Para todo $v=\left(v_{i}\right)_{i \in I} \in \bar{\bigoplus}_{i \in I}^{1} E_{i}$ temos que $|\alpha(v)| \leq\|\alpha\|\|v\|_{1}$. Assim, se $u \in E_{i}$ então $|\alpha|_{E_{i}}(u)|=| \alpha(u) \mid \leq\|\alpha\|\|u\|_{1}=\|\alpha\|\|u\| ; \operatorname{logo}$ $\left\|\left.\alpha\right|_{E_{i}}\right\| \leq\|\alpha\|$ para cada $i \in I$. Portanto

$$
\left\|\left(\left.\alpha\right|_{E_{i}}\right)_{i \in I}\right\|_{\infty}=\sup _{i \in I}\left\|\left.\alpha\right|_{E_{i}}\right\| \leq\|\alpha\| \text {, i.e., } \quad\left(\left.\alpha\right|_{E_{i}}\right)_{i \in I} \in \bar{\bigoplus}_{i \in I}^{\infty} E_{i}^{*} .
$$

- $1<p<+\infty$ e $q$ dado por $\frac{1}{p}+\frac{1}{q}=1$.

Seja $F$ um subconjunto finito de $I$. Dado $0<\varepsilon<1$, para cada $i \in F$ escolhemos $v_{i} \in E_{i}$ com $\left\|v_{i}\right\| \leq 1$, e tal que $\left|\alpha\left(v_{i}\right)\right| \geq(1-\varepsilon)\left\|\left.\alpha\right|_{E_{i}}\right\|$. Para cada $i \in F$, seja $x_{i}=\left\|\left.\alpha\right|_{E_{i}}\right\|^{\frac{q}{p}} \operatorname{sgn}\left(\alpha\left(v_{i}\right)\right) v_{i} \in E_{i}$, e tomemos $x=\sum_{i \in F} x_{i} \in \bar{\bigoplus}_{i \in I}^{p} E_{i}$.

$$
\text { Assim, } \begin{aligned}
|\alpha(x)|=\left|\sum_{i \in F} \alpha\left(x_{i}\right)\right| & =\sum_{i \in F}\left\|\left.\alpha\right|_{E_{i}}\right\|^{\frac{q}{p}}\left|\alpha\left(v_{i}\right)\right| \\
& \geq \sum_{i \in F}\left\|\left.\alpha\right|_{E_{i}}\right\|^{\frac{q}{p}}(1-\varepsilon)\left\|\left.\alpha\right|_{E_{i}}\right\| \\
& =(1-\varepsilon) \sum_{i \in F}\left\|\left.\alpha\right|_{E_{i}}\right\|^{q} .
\end{aligned}
$$

Por outro lado, $\quad|\alpha(x)| \leq\|\alpha\|\|x\|_{p}$

$$
\begin{aligned}
& =\|\alpha\|\left(\sum_{i \in I}\left\|x_{i}\right\|^{p}\right)^{\frac{1}{p}} \\
& =\|\alpha\|\left(\left.\sum_{i \in F}\|\| \alpha\right|_{E_{i}}\left\|^{\frac{q}{p}} \operatorname{sgn}\left(\alpha\left(v_{i}\right)\right) v_{i}\right\|^{p}\right)^{\frac{1}{p}} \\
& =\|\alpha\|\left(\sum_{i \in F}\left\|\left.\alpha\right|_{E_{i}}\right\|^{q}\left\|v_{i}\right\|^{p}\right)^{\frac{1}{p}} \\
& \leq\|\alpha\|\left(\sum_{i \in F}\left\|\left.\alpha\right|_{E_{i}}\right\|^{q}\right)^{\frac{1}{p}}
\end{aligned}
$$

uma vez que $\left\|v_{i}\right\| \leq 1$ para cada $i \in F$. Portanto,

$$
(1-\varepsilon) \sum_{i \in F}\left\|\left.\alpha\right|_{E_{i}}\right\|^{q} \leq|\alpha(x)| \leq\|\alpha\|\left(\sum_{i \in F}\left\|\left.\alpha\right|_{E_{i}}\right\|^{q}\right)^{\frac{1}{p}} .
$$


Se $\sum_{i \in F}\left\|\left.\alpha\right|_{E_{i}}\right\|^{q} \neq 0$ então $(1-\varepsilon)\left(\sum_{i \in F}\left\|\left.\alpha\right|_{E_{i}}\right\|^{q}\right)^{1-\frac{1}{p}} \leq\|\alpha\|$, ou seja,

$$
(1-\varepsilon)\left(\sum_{i \in F}\left\|\left.\alpha\right|_{E_{i}}\right\|^{q}\right)^{\frac{1}{q}} \leq\|\alpha\| .
$$

Observe que (3.2.2) também é válida se $\sum_{i \in F}\left\|\left.\alpha\right|_{E_{i}}\right\|^{q}=0$. Assim, uma vez que (3.2.2) é verdadeira para todo $\varepsilon \in] 0,1[$, e para todo $F \subset I$ finito, temos:

$$
\left\|\left(\left.\alpha\right|_{E_{i}}\right)_{i \in I}\right\|_{q}=\left(\sum_{i \in I}\left\|\left.\alpha\right|_{E_{i}}\right\|^{q}\right)^{\frac{1}{q}} \leq\|\alpha\| .
$$

Portanto $\left(\left.\alpha\right|_{E_{i}}\right)_{i \in I} \in \bar{\bigoplus}_{i \in I}^{q} E_{i}^{*}$.

- $p=+\infty$ e $q=1$.

Seja $F \subset I$ finito. Dado $\varepsilon \in] 0,1[$, para cada $i \in F$ escolhemos $v_{i} \in E_{i}$ com $\left\|v_{i}\right\| \leq 1$, e tal que $\left|\alpha\left(v_{i}\right)\right| \geq(1-\varepsilon)\left\|\left.\alpha\right|_{E_{i}}\right\|$. Para cada $i \in F$, seja $x_{i}=\operatorname{sgn}\left(\alpha\left(v_{i}\right)\right) v_{i} \in E_{i}$, e tomemos $x=\sum_{i \in F} x_{i} \in \bar{\bigoplus}_{i \in I}^{\infty} E_{i}$. Assim, temos:

$$
|\alpha(x)|=\left|\sum_{i \in F} \alpha\left(x_{i}\right)\right|=\left|\sum_{i \in F}\right| \alpha\left(v_{i}\right)||=\sum_{i \in F}\left|\alpha\left(v_{i}\right)\right| \geq(1-\varepsilon) \sum_{i \in F}\left\|\left.\alpha\right|_{E_{i}}\right\| .
$$

Por outro lado,

$$
|\alpha(x)| \leq\|\alpha\|\|x\|_{\infty}=\|\alpha\| \sup _{i \in F}\left\|x_{i}\right\|=\|\alpha\| \sup _{i \in F}\left\|v_{i}\right\| \leq\|\alpha\| 1=\|\alpha\| .
$$

Portanto, temos $(1-\varepsilon) \sum_{i \in F}\left\|\left.\alpha\right|_{E_{i}}\right\| \leq\|\alpha\|$ para todo $\left.\varepsilon \in\right] 0,1[$ e para todo $F \subset I$ finito, o que implica em

$$
\left\|\left(\left.\alpha\right|_{E_{i}}\right)_{i \in I}\right\|_{1}=\sum_{i \in I}\left\|\left.\alpha\right|_{E_{i}}\right\| \leq\|\alpha\|
$$

ou seja, $\left(\left.\alpha\right|_{E_{i}}\right)_{i \in I} \in \bar{\bigoplus}_{i \in I}^{1} E_{i}^{*}$.

Vamos mostrar agora que se $p<+\infty$ então $\alpha(v)=\sum_{i \in I} \alpha\left(v_{i}\right)$ para todo $v=\left(v_{i}\right)_{i \in I} \in \bar{\bigoplus}_{i \in I}^{p} E_{i}$. Mostraremos preliminarmente que a família $v=\left(v_{i}\right)_{i \in I}$ é somável e $v=\sum_{i \in I} v_{i}$. Seja $\varepsilon>0$ dado. Se $p<+\infty$ e $v=\left(v_{i}\right)_{i \in I} \in \bar{\bigoplus}_{i \in I}^{p} E_{i}$ então $\sum_{i \in I}\left\|v_{i}\right\|^{p}<+\infty$; logo, existe $F_{\varepsilon} \subset I$ finito tal 
que, para todo $F \supset F_{\varepsilon}$ finito temos $\sum_{i \in I \backslash F}\left\|v_{i}\right\|^{p}<\varepsilon^{p}$. Assim,

$$
\begin{array}{r}
\left\|v-\sum_{i \in F} v_{i}\right\|_{p}=\left\|v-\sum_{i \in I} v_{i} \chi_{F}(i)\right\|_{p}=\left\|\left(v_{i}\right)_{i \in I}-\left(v_{i} \chi_{F}(i)\right)_{i \in I}\right\|_{p} \\
=\left\|\left(v_{i}-v_{i} \chi_{F}(i)\right)_{i \in I}\right\|_{p}=\left(\sum_{i \in I \backslash F}\left\|v_{i}\right\|^{p}\right)^{\frac{1}{p}},
\end{array}
$$

isto é, $\left\|v-\sum_{i \in F} v_{i}\right\|_{p}<\varepsilon$. Portanto $v=\left(v_{i}\right)_{i \in I}$ é somável e $v=\sum_{i \in I} v_{i}$. O resultado segue diretamente da Proposição 1.3.10.

Resta mostrar que se $p<+\infty$ então é uma isometria linear a aplicação

$$
\Phi:\left(\bar{\bigoplus}_{i \in I}^{p} E_{i}\right)^{*} \ni \alpha \longmapsto\left(\left.\alpha\right|_{E_{i}}\right)_{i \in I} \in \bar{\bigoplus}_{i \in I}^{q} E_{i}^{*} .
$$

$\Phi$ é claramente linear, e já vimos que para $p \in\left[1,+\infty\left[\right.\right.$ e $q$ dado por $\frac{1}{p}+\frac{1}{q}=1$, temos que $\|\Phi(\alpha)\|_{q}=\left\|\left(\left.\alpha\right|_{E_{i}}\right)_{i \in I}\right\|_{q} \leq\|\alpha\|$ para todo $\alpha \in\left(\bar{\bigoplus}_{i \in I}^{p} E_{i}\right)^{*}$. Portanto, para mostrar que $\Phi$ é uma imersão isométrica, basta mostrar que $\|\alpha\| \leq\left\|\left(\left.\alpha\right|_{E_{i}}\right)_{i \in I}\right\|_{q}$ para cada funcional linear limitado sobre $\bar{\bigoplus}_{i \in I}^{p} E_{i}$. Seja $v=\left(v_{i}\right)_{i \in I} \in \bar{\bigoplus}_{i \in I}^{p} E_{i}$ arbitrário. Pelo Lema 3.2.7 temos:

$$
|\alpha(v)|=\left|\sum_{i \in I} \alpha\left(v_{i}\right)\right|=\left|\sum_{i \in I} \alpha\right|_{E_{i}}\left(v_{i}\right) \mid \leq\left\|\left(\left.\alpha\right|_{E_{i}}\right)_{i \in I}\right\|_{q}\left\|\left(v_{i}\right)_{i \in I}\right\|_{p} .
$$

ou seja, $|\alpha(v)| \leq\left\|\left(\left.\alpha\right|_{E_{i}}\right)_{i \in I}\right\|_{q}\|v\|_{p}$ para todo $v \in \bar{\bigoplus}_{i \in I}^{p} E_{i}$. Portanto

$$
\|\alpha\| \leq\left\|\left(\left.\alpha\right|_{E_{i}}\right)_{i \in I}\right\|_{q}
$$

uma vez que $\|\alpha\|=\inf \left\{c \geq 0:|\alpha(v)| \leq c\|v\|_{p}\right.$, para todo $\left.v \in \bar{\bigoplus}_{i \in I}^{p} E_{i}\right\}$. Para finalizar, vamos mostrar que $\Phi$ é sobrejetora. Dada uma família arbitrária $\left(\alpha_{i}\right)_{i \in I} \in \bar{\bigoplus}_{i \in I}^{q} E_{i}^{*}$, consideremos a aplicação $\alpha: \bar{\bigoplus}_{i \in I}^{p} E_{i} \rightarrow \mathbb{R}$ dada por $\alpha(v)=\sum_{i \in I} \alpha_{i}\left(v_{i}\right)$, para cada $v=\left(v_{i}\right)_{i \in I} \in \bar{\bigoplus}_{i \in I}^{p} E_{i}$. A aplicação $\alpha$ é claramente linear e está bem definida, uma vez que pelo Lema 3.2.7 temos

$$
\sum_{i \in I}\left|\alpha_{i}\left(v_{i}\right)\right| \leq\left\|\left(\alpha_{i}\right)_{i \in I}\right\|_{q}\|v\|_{p}<+\infty .
$$

Assim, a família $\left(\alpha_{i}\left(v_{i}\right)\right)_{i \in I}$ é absolutamente somável, portanto somável. Além disso, $|\alpha(v)| \leq\left\|\left(\alpha_{i}\right)_{i \in I}\right\|_{q}\|v\|_{p}$ para todo $v=\left(v_{i}\right)_{i \in I} \in \bar{\bigoplus}_{i \in I}^{p} E_{i}$, i.e., $\alpha \in\left(\bar{\bigoplus}_{i \in I}^{p} E_{i}\right)^{*}$. É fácil ver que $\alpha_{i}=\left.\alpha\right|_{E_{i}}$ para cada $i \in I$, logo $\Phi$ leva $\alpha$ em $\left(\alpha_{i}\right)_{i \in I}$. Portanto $\Phi$ é sobrejetora, e isso completa a demonstração. 
Proposição 3.2.9. Se $\left(X_{i}, \mathcal{A}_{i}, \mu_{i}\right)_{i \in I}$ é uma família de espaços de medida então, para todo $p \in[1,+\infty]$, a aplicação

$$
\Phi: L^{p}\left(\sum_{i \in I}\left(X_{i}, \mathcal{A}_{i}, \mu_{i}\right)\right) \ni f \longmapsto\left(\left.f\right|_{X_{i}}\right)_{i \in I} \in \bar{\bigoplus}_{i \in I}^{p} L^{p}\left(X_{i}, \mathcal{A}_{i}, \mu_{i}\right)
$$

é uma isometria linear.

DemonstraÇão. Seja $(X, \mathcal{A}, \mu)=\sum_{i \in I}\left(X_{i}, \mathcal{A}_{i}, \mu_{i}\right)$. Afirmamos que

$$
\|f\|_{p}=\left\|\left(\left\|\left.f\right|_{X_{i}}\right\|_{p}\right)_{i \in I}\right\|_{p}
$$

para todo $p \in[1,+\infty]$ e para cada $f: X \rightarrow \mathbb{R}$ mensurável. Com efeito, se $p \in[1,+\infty[$, pela Proposição 3.1 .5 temos

$$
\begin{aligned}
\|f\|_{p} & =\left(\int_{X}|f|^{p} \mathrm{~d} \mu\right)^{\frac{1}{p}}=\left(\sum_{i \in I} \int_{X_{i}}|f|^{p} \mathrm{~d} \mu\right)^{\frac{1}{p}} \\
& =\left(\left.\sum_{i \in I} \int_{X_{i}}|f|_{X_{i}}\right|^{p} \mathrm{~d} \mu_{i}\right)^{\frac{1}{p}}=\left(\sum_{i \in I}\left\|\left.f\right|_{X_{i}}\right\|_{p}^{p}\right)^{\frac{1}{p}}=\left\|\left(\left\|\left.f\right|_{X_{i}}\right\|_{p}\right)_{i \in I}\right\|_{p} .
\end{aligned}
$$

Seja agora $p=+\infty$. Sejam $i \in I$ fixado e $c$ um número real não negativo. Se $|f| \leq c \mu$-qs então

$$
\begin{aligned}
& \mu_{i}\left(\left\{x \in X_{i}:|f|_{X_{i}}(x) \mid>c\right\}\right)=\mu_{i}\left(\{x \in X:|f(x)|>c\} \cap X_{i}\right) \\
& \quad \leq \sum_{i \in I} \mu_{i}\left(\{x \in X:|f(x)|>c\} \cap X_{i}\right)=\mu(\{x \in X:|f(x)|>c\})=0
\end{aligned}
$$

ou seja, $|f|_{X_{i}} \mid \leq c \mu_{i}$-qs; portanto se $A \stackrel{\text { def }}{=}\{c \geq 0:|f| \leq c \mu$-qs $\}$ então

$$
A_{i} \stackrel{\text { def }}{=}\left\{c \geq 0:|f|_{X_{i}} \mid \leq c \mu_{i} \text {-qs }\right\} \supset A .
$$

Assim, $\left\|\left.f\right|_{X_{i}}\right\|_{\infty}=\inf A_{i} \leq \inf A=\|f\|_{\infty}$ para todo $i \in I$, e daí

$$
\left\|\left(\left\|\left.f\right|_{X_{i}}\right\|_{\infty}\right)_{i \in I}\right\|_{\infty}=\sup _{i \in I}\left\|\left.f\right|_{X_{i}}\right\|_{\infty} \leq\|f\|_{\infty} .
$$

Por outro lado, para cada $i \in I$, temos que $|f|_{X_{i}}\left|\leq\left\|\left.f\right|_{X_{i}}\right\|_{\infty} \mu_{i}\right.$-qs, isto é, $|f| \leq\left\|\left.f\right|_{X_{i}}\right\|_{\infty} \mu_{i}$-qs em $X_{i}$. Logo, existe $A_{i} \in \mathcal{A}_{i}$ com $\mu_{i}\left(A_{i}\right)=0$ tal que $|f| \leq\left\|\left.f\right|_{X_{i}}\right\|_{\infty}$ em $X_{i} \backslash A_{i}$, e portanto $|f| \leq \sup _{i \in I}\left\|\left.f\right|_{X_{i}}\right\|_{\infty} \operatorname{em} \bigcup_{i \in I}\left(X_{i} \backslash A_{i}\right)$. Observe que, uma vez que os conjuntos $X_{i}$ são dois a dois disjuntos, temos

$$
\bigcup_{i \in I}\left(X_{i} \backslash A_{i}\right)=\bigcup_{i \in I} X_{i} \backslash \bigcup_{i \in I} A_{i}
$$


Assim, $|f(x)| \leq \sup _{i \in I}\left\|\left.f\right|_{X_{i}}\right\|_{\infty}$ para todo $x \in X \backslash \bigcup_{i \in I} A_{i}$ e

$$
\mu\left(\bigcup_{j \in I} A_{j}\right)=\sum_{i \in I} \mu_{i}\left(\left(\bigcup_{j \in I} A_{j}\right) \cap X_{i}\right)=\sum_{i \in I} \mu_{i}\left(A_{i}\right)=0,
$$

ou seja,

$$
|f| \leq \sup _{i \in I}\left\|\left.f\right|_{X_{i}}\right\|_{\infty}=\left\|\left(\left\|\left.f\right|_{X_{i}}\right\|_{\infty}\right)_{i \in I}\right\|_{\infty} \mu \text {-qs } .
$$

Como $\|f\|_{\infty}=\inf A$, segue que $\|f\|_{\infty} \leq\left\|\left(\left\|\left.f\right|_{X_{i}}\right\|_{\infty}\right)_{i \in I}\right\|_{\infty}$, e assim

$$
\|f\|_{\infty}=\left\|\left(\left\|\left.f\right|_{X_{i}}\right\|_{\infty}\right)_{i \in I}\right\|_{\infty} .
$$

Vamos mostrar agora que a aplicação (3.2.3) está bem definida. Sejam $f, g \in L^{p}(X, \mathcal{A}, \mu)$ com $f=g \mu$-qs. Para cada $i \in I$ temos que

$$
\begin{aligned}
& \mu_{i}\left(\left\{x \in X_{i}:\left.f\right|_{X_{i}}(x) \neq\left. g\right|_{X_{i}}(x)\right\}\right)=\mu_{i}\left(\{x \in X: f(x) \neq g(x)\} \cap X_{i}\right) \\
& \leq \sum_{i \in I} \mu_{i}\left(\{x \in X: f(x) \neq g(x)\} \cap X_{i}\right)=\mu(\{x \in X: f(x) \neq g(x)\})=0,
\end{aligned}
$$

isto é, $\left.f\right|_{X_{i}}=\left.g\right|_{X_{i}} \mu_{i}$-qs. Isso mostra que a imagem da classe $[f]_{\mu}$ pela aplicação $\Phi$ independe do representante tomado. Sejam $f \in L^{p}(X, \mathcal{A}, \mu)$ e $p \in[1,+\infty]$. Se $f$ é mensurável relativamente a $\mathcal{A}$ então, pela Observação 3.1.2, $\left.f\right|_{X_{i}}$ é $\mathcal{A}_{i}$-mensurável para cada $i \in I$. Além disso,

$$
\left\|\left(\left.f\right|_{X_{i}}\right)_{i \in I}\right\|_{p}=\left\|\left(\left\|\left.f\right|_{X_{i}}\right\|_{p}\right)_{i \in I}\right\|_{p}=\|f\|_{p}<+\infty
$$

logo, $\left.f\right|_{X_{i}} \in L^{p}\left(X_{i}, \mathcal{A}_{i}, \mu_{i}\right)$ para todo $i \in I$ e $\left(\left.f\right|_{X_{i}}\right)_{i \in I} \in \bar{\bigoplus}_{i \in I}^{p} L^{p}\left(X_{i}, \mathcal{A}_{i}, \mu_{i}\right)$, o que mostra que $\Phi$ está bem definida. Observe que $\Phi$ é claramente linear e de $\left\|\left(\left.f\right|_{X_{i}}\right)_{i \in I}\right\|_{p}=\|f\|_{p}$ segue que $\Phi$ é uma imersão isométrica. Resta mostrar a sobrejetividade de (3.2.3). Seja $\left(f_{i}\right)_{i \in I}$ uma família de funções em $\bar{\bigoplus}_{i \in I}^{p} L^{p}\left(X_{i}, \mathcal{A}_{i}, \mu_{i}\right)$, e consideremos a função $f: X \rightarrow \mathbb{R}$ definida por $\left.f\right|_{X_{i}}=f_{i}$ para cada $i \in I$. Uma vez que $\left.f\right|_{X_{i}}$ é $\mathcal{A}_{i}$-mensurável para todo $i \in I$, pela Observação 3.1.2, a função $f$ é mensurável com respeito a $\mathcal{A}$. Ainda, como $\|f\|_{p}=\left\|\left(\left.f\right|_{X_{i}}\right)_{i \in I}\right\|_{p}=\left\|\left(f_{i}\right)_{i \in I}\right\|_{p}<+\infty$, temos $f \in L^{p}(X, \mathcal{A}, \mu)$. Obviamente $\Phi(f)=\left(f_{i}\right)_{i \in I}$, e isso conclui a prova de que $\Phi$ é sobrejetora.

Se $p \in[1,+\infty[, q \in] 1,+\infty]$ e $\frac{1}{p}+\frac{1}{q}=1$ então a composição da transposta da isometria linear (3.2.3) com a inversa da isometria linear (3.2.1) dá-nos a seguinte isometria linear:

$$
\bar{\bigoplus}_{i \in I}^{q} L^{p}\left(X_{i}, \mathcal{A}_{i}, \mu_{i}\right)^{*} \ni\left(\alpha_{i}\right)_{i \in I} \longmapsto \alpha \in L^{p}\left(\sum_{i \in I}\left(X_{i}, \mathcal{A}_{i}, \mu_{i}\right)\right)^{*},
$$


na qual $\left(X_{i}, \mathcal{A}_{i}, \mu_{i}\right)_{i \in I}$ é uma família arbitrária de espaços de medida e $\alpha$ é dado por $\alpha(f)=\sum_{i \in I} \alpha_{i}\left(\left.f\right|_{X_{i}}\right)$, para todo $f \in L^{p}\left(\sum_{i \in I}\left(X_{i}, \mathcal{A}_{i}, \mu_{i}\right)\right)$. Com efeito, para a família de espaços de Banach $\left(L^{p}\left(X_{i}, \mathcal{A}_{i}, \mu_{i}\right)\right)_{i \in I}$ temos o seguinte diagrama

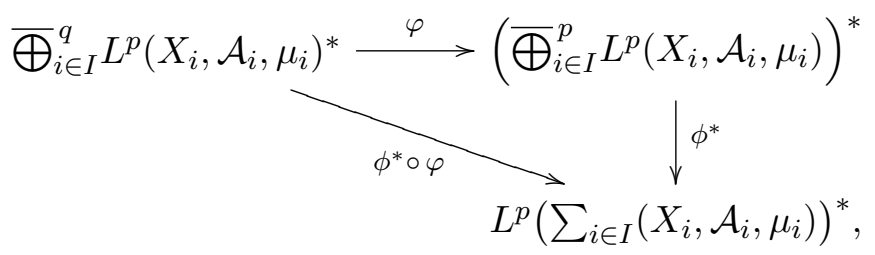

no qual e $\phi^{*}$ é a transposta da aplicação (3.2.3) e $\varphi$ é a inversa da aplicação (3.2.1), dada por $\varphi\left(\left(\alpha_{i}\right)_{i \in I}\right)=\beta$, tal que $\left.\beta\right|_{L^{p}\left(X_{i}, \mathcal{A}_{i}, \mu_{i}\right)}=\alpha_{i}$ para cada $i \in I$, e $\beta(h)=\sum_{i \in I} \alpha_{i}\left(h_{i}\right)$ para todo $h=\left(h_{i}\right)_{i \in I} \in \bar{\bigoplus}_{i \in I}^{p} L^{p}\left(X_{i}, \mathcal{A}_{i}, \mu_{i}\right)$. Para cada família $\left(\alpha_{i}\right)_{i \in I}$ em $\bar{\bigoplus}_{i \in I}^{q} L^{p}\left(X_{i}, \mathcal{A}_{i}, \mu_{i}\right)^{*}$ e $f \in L^{p}\left(\sum_{i \in I}\left(X_{i}, \mathcal{A}_{i}, \mu_{i}\right)\right)$ temos:

$$
\begin{aligned}
&\left(\phi^{*} \circ \varphi\right)\left(\left(\alpha_{i}\right)_{i \in I}\right)(f)=\left(\phi^{*}\left(\varphi\left(\left(\alpha_{i}\right)_{i \in I}\right)\right)\right)(f)=\left(\phi^{*}(\beta)\right)(f) \\
&=(\beta \circ \phi)(f)=\beta(\phi(f))=\beta\left(\left(\left.f\right|_{X_{i}}\right)_{i \in I}\right)=\sum_{i \in I} \alpha_{i}\left(\left.f\right|_{X_{i}}\right)=\alpha(f) .
\end{aligned}
$$

Portanto a isometria $\phi^{*} \circ \varphi$ leva a família $\left(\alpha_{i}\right)_{i \in I}$ no funcional linear $\alpha$. Como conseqüência, é comutativo o diagrama

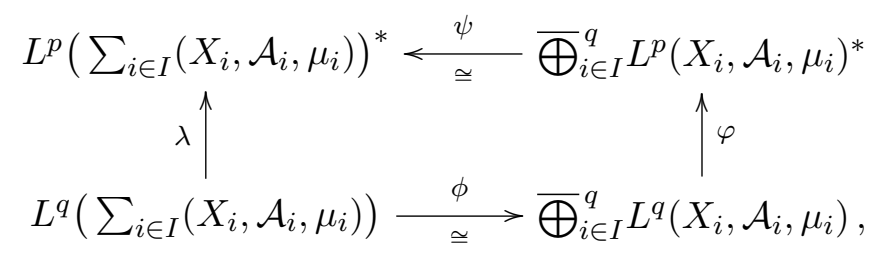

no qual $\psi$ é a aplicação (3.2.4), $\phi$ é a versão da aplicação (3.2.3) para $L^{q}, \lambda$ é a $(q, p)$-aplicação de Riesz (1.4.2) para o espaço $\sum_{i \in I}\left(X_{i}, \mathcal{A}_{i}, \mu_{i}\right)$ e $\varphi$ é a aplicação dada pela $(q, p)$-aplicação de Riesz (1.4.2) para o espaço $\left(X_{i}, \mathcal{A}_{i}, \mu_{i}\right)$ em cada coordenada. De fato, seja $g \in L^{q}\left(\sum_{i \in I}\left(X_{i}, \mathcal{A}_{i}, \mu_{i}\right)\right)$ fixada. Então $\lambda(g)=\lambda_{g} \in L^{p}\left(\sum_{i \in I}\left(X_{i}, \mathcal{A}_{i}, \mu_{i}\right)\right)^{*}$ é tal que

$$
\lambda_{g}(f)=\int_{\sum_{i \in I} X_{i}} f g \mathrm{~d} \mu
$$

para toda $f \in L^{p}\left(\sum_{i \in I}\left(X_{i}, \mathcal{A}_{i}, \mu_{i}\right)\right)$. Por outro lado, a aplicação $\phi$ leva $g$ na família $\left(\left.g\right|_{X_{i}}\right)_{i \in I} \in \bar{\bigoplus}_{i \in I}^{q} L^{q}\left(X_{i}, \mathcal{A}_{i}, \mu_{i}\right)$, que por sua vez é levada por $\varphi$ 
em $\left(\beta_{\left.g\right|_{X_{i}}}\right)_{i \in I} \in \bar{\bigoplus}_{i \in I}^{q} L^{p}\left(X_{i}, \mathcal{A}_{i}, \mu_{i}\right)^{*}$, na qual

$$
\beta_{\left.g\right|_{X_{i}}}\left(f_{i}\right)=\left.\int_{X_{i}} f_{i} g\right|_{X_{i}} \mathrm{~d} \mu_{i}
$$

para cada $i \in I$ e para toda $f_{i} \in L^{p}\left(X_{i}, \mathcal{A}_{i}, \mu_{i}\right)$. Por fim, aplicando $\psi$ na família $\left(\beta_{\left.g\right|_{X_{i}}}\right)_{i \in I}$ obtemos $\beta \in L^{p}\left(\sum_{i \in I}\left(X_{i}, \mathcal{A}_{i}, \mu_{i}\right)\right)^{*}$ dado por

$$
\beta(f)=\sum_{i \in I} \beta_{\left.g\right|_{X_{i}}}\left(\left.f\right|_{X_{i}}\right),
$$

para toda $f \in L^{p}\left(\sum_{i \in I}\left(X_{i}, \mathcal{A}_{i}, \mu_{i}\right)\right)$. Portanto, para cada função $f \mathrm{em}$ $L^{p}\left(\sum_{i \in I}\left(X_{i}, \mathcal{A}_{i}, \mu_{i}\right)\right)$ temos ( pela Proposição 3.1.5):

$$
\beta(f)=\sum_{i \in I} \int_{X_{i}}\left(\left.\left.f\right|_{X_{i}} \cdot g\right|_{X_{i}}\right) \mathrm{d} \mu_{i}=\sum_{i \in I} \int_{X_{i}} f g \mathrm{~d} \mu=\int_{\sum_{i \in I} X_{i}} f g \mathrm{~d} \mu=\lambda_{g}(f),
$$

ou seja, $\beta=\lambda_{g}$. Portanto, $(\psi \circ \varphi \circ \phi)(g)=\beta=\lambda_{g}=\lambda(g)$ para cada $g \in L^{q}\left(\sum_{i \in I}\left(X_{i}, \mathcal{A}_{i}, \mu_{i}\right)\right)$, o que mostra que o diagrama (3.2.5) comuta. 


\section{CAPíTULO 4}

\section{Decomposição de um espaço de medida e o Teorema de Riesz}

\subsection{Decomposições essenciais e decomposições}

Definição 4.1.1. Seja $(X, \mathcal{A}, \mu)$ um espaço de medida. Uma família $\left(X_{i}\right)_{i \in I}$ de subconjuntos mensuráveis de $X$ é chamada de essencialmente disjunta se $\mu\left(X_{i} \cap X_{j}\right)=0$, para todo $i, j \in I$ com $i \neq j$. Uma decomposição essencial para $(X, \mathcal{A}, \mu)$ é uma família essencialmente disjunta $\left(X_{i}\right)_{i \in I}$ de subconjuntos mensuráveis de $X$ que satisfaz as seguintes propriedades:

- $0<\mu\left(X_{i}\right)<+\infty$, para todo $i \in I$;

- se $A \in \mathcal{A}, \mu(A)<+\infty$ e $\mu\left(A \cap X_{i}\right)=0$ para todo $i \in I$ então $\mu(A)=0$.

Uma decomposição essencial $\left(X_{i}\right)_{i \in I}$ para $(X, \mathcal{A}, \mu)$, na qual os conjuntos $X_{i}$ são dois a dois disjuntos, é chamada de uma decomposição para $(X, \mathcal{A}, \mu)$.

Lema 4.1.2. $S e(X, \mathcal{A}, \mu)$ é um espaço de medida e $\left(A_{i}\right)_{i \in I}$ é uma família essencialmente disjunta enumerável de elementos de $\mathcal{A}$ então:

$$
\mu\left(\bigcup_{i \in I} A_{i}\right)=\sum_{i \in I} \mu\left(A_{i}\right)
$$

Demonstração. Podemos supor $I=\omega \backslash\{0\}$. Seja $\left(B_{i}\right)_{i \in I}$ tal que $B_{1}=A_{1}$ e $B_{i}=A_{i} \backslash \bigcup_{k=1}^{i-1} A_{k}$ para todo $i \geq 2$. Pela Proposição 1.4.12, $B_{i} \in \mathcal{A}$ para todo $i \in I, B_{i} \cap B_{j}=\emptyset$ se $i \neq j$ e $\bigcup_{i \in I} B_{i}=\bigcup_{i \in I} A_{i}$. Afirmamos que $\mu\left(A_{i}\right)=\mu\left(B_{i}\right)$ para todo $i \in I$. Obviamente $\mu\left(A_{1}\right)=\mu\left(B_{1}\right)$, e

$\mu\left(B_{i}\right)=\mu\left(A_{i} \backslash \bigcup_{k=1}^{i-1} A_{k}\right)=\mu\left(A_{i} \backslash\left(\left(\bigcup_{k=1}^{i-1} A_{k}\right) \cap A_{i}\right)\right)=\mu\left(A_{i} \backslash \bigcup_{k=1}^{i-1}\left(A_{k} \cap A_{i}\right)\right)$,

para $i \geq 2$. Como $\left(A_{i}\right)_{i \in I}$ é uma família essencialmente disjunta, temos

$$
\mu\left(\bigcup_{k=1}^{i-1}\left(A_{k} \cap A_{i}\right)\right) \leq \sum_{k=1}^{i-1} \mu\left(A_{k} \cap A_{i}\right)=0<+\infty .
$$


Logo $\mu\left(B_{i}\right)=\mu\left(A_{i}\right)-\mu\left(\bigcup_{k=1}^{i-1}\left(A_{k} \cap A_{i}\right)\right)=\mu\left(A_{i}\right)$. Portanto,

$$
\mu\left(\bigcup_{i \in I} A_{i}\right)=\mu\left(\bigcup_{i \in I} B_{i}\right)=\sum_{i \in I} \mu\left(B_{i}\right)=\sum_{i \in I} \mu\left(A_{i}\right)
$$

Exemplo 4.1.3. Seja $(X, \mathcal{A}, \mu)$ um espaço de medida. É claro que toda família $\left(X_{i}\right)_{i \in I}$ de subconjuntos mensuráveis de $X$, dois a dois disjuntos, é uma família essencialmente disjunta. Se $\left(X_{i}\right)_{i \in I}$ é uma família enumerável essencialmente disjunta de subconjuntos mensuráveis de $X$, de medida finita positiva, tal que $X=\bigcup_{i \in I} X_{i}$, então $\left(X_{i}\right)_{i \in I}$ é uma decomposição essencial para $X$. Com efeito, seja $A \in \mathcal{A} \operatorname{com} \mu(A)<+\infty$ e $\mu\left(A \cap X_{i}\right)=0$ para todo $i \in I$. Observe que para $i, j \in I$ com $i \neq j$ temos

$$
\mu\left(\left(A \cap X_{i}\right) \cap\left(A \cap X_{j}\right)\right)=\mu\left(A \cap\left(X_{i} \cap X_{j}\right)\right) \leq \mu\left(X_{i} \cap X_{j}\right)=0,
$$

ou seja, $\left(A \cap X_{i}\right)_{i \in I}$ é uma família essencialmente disjunta de subconjuntos mensuráveis de $X$. Então

$\mu(A)=\mu(A \cap X)=\mu\left(A \cap\left(\bigcup_{i \in I} X_{i}\right)\right)=\mu\left(\bigcup_{i \in I}\left(A \cap X_{i}\right)\right)=\sum_{i \in I} \mu\left(A \cap X_{i}\right)=0$.

Em particular, se $(X, \mathcal{A}, \mu)$ é $\sigma$-finito então existe uma família $\left(X_{i}\right)_{i \in I}$ de subconjuntos mensuráveis de $X$ de medida positiva finita, dois a dois disjuntos, com $|I| \leq \aleph_{0}$ e $X=\bigcup_{i \in I} X_{i}$; logo $\left(X_{i}\right)_{i \in I}$ é uma decomposição para o espaço $(X, \mathcal{A}, \mu)$.

Exemplo 4.1.4. Se $\left(X_{i}, \mathcal{A}_{i}, \mu_{i}\right)_{i \in I}$ é uma família de espaços de medida, com $0<\mu_{i}\left(X_{i}\right)<+\infty$ para todo $i \in I$, então a família $\left(X_{i}\right)_{i \in I}$ é uma decomposição para a soma externa $(X, \mathcal{A}, \mu)=\sum_{i \in I}\left(X_{i}, \mathcal{A}_{i}, \mu_{i}\right)$. De fato, se $A \in \mathcal{A} \operatorname{com} \mu(A)<+\infty$ e $\mu\left(A \cap X_{i}\right)=0$ para todo $i \in I$, então $A \cap X_{i} \in \mathcal{A}_{i}$ e $\mu\left(A \cap X_{i}\right)=\mu_{i}\left(A \cap X_{i}\right)$ para cada $i \in I, \operatorname{logo} \mu(A)=\sum_{i \in I} \mu_{i}\left(A \cap X_{i}\right)=0$.

Lema 4.1.5. Sejam $(X, \mathcal{A}, \mu)$ um espaço de medida e $\left(X_{i}\right)_{i \in I}$ uma família essencialmente disjunta de subconjuntos mensuráves de $X$. Se $A \in \mathcal{A}$ é $\sigma$-finito para $\mu$ então o conjunto $J=\left\{i \in I: \mu\left(A \cap X_{i}\right)>0\right\}$ é enumerável.

Demonstração. Consideremos a princípio o caso em que $\mu(A)<+\infty$. Se $\mu(A)<+\infty$ então, para todo $\varepsilon>0$, o conjunto $\left\{i \in I: \mu\left(A \cap X_{i}\right) \geq \varepsilon\right\}$ é 
finito, do contrário admitiria um subconjunto infinito enumerável $I^{\prime}$ tal que

$$
\begin{aligned}
\mu(A) \geq \mu\left(A \cap\left(\bigcup_{i \in I} X_{i}\right)\right)=\mu\left(\bigcup_{i \in I}\left(A \cap X_{i}\right)\right) & \geq \mu\left(\bigcup_{i \in I^{\prime}}\left(A \cap X_{i}\right)\right) \\
& =\sum_{i \in I^{\prime}} \mu\left(A \cap X_{i}\right)=+\infty .
\end{aligned}
$$

Observe que a penúltima igualdade acima é garantida pelo Lema 4.1.2, uma vez que $\left(A \cap X_{i}\right)_{i \in I^{\prime}}$ é uma família essencialmente disjunta enumerável. Como

$$
J=\bigcup_{k \geq 1}\left\{i \in I: \mu\left(A \cap X_{i}\right) \geq \frac{1}{k}\right\}
$$

segue que $J$ é enumerável. Suponhamos agora que $A$ é $\sigma$-finito para $\mu$. Então existe uma seqüência $\left(A_{k}\right)_{k \geq 1}$ de subconjuntos mensuráveis de $X$, com $\mu\left(A_{k}\right)<+\infty$ para todo $k \geq 1$, tal que $A=\bigcup_{k \geq 1} A_{k}$. Assim,

$$
J=\left\{i \in I: \mu\left(\left(\bigcup_{k \geq 1} A_{k}\right) \cap X_{i}\right)>0\right\}=\left\{i \in I: \mu\left(\bigcup_{k \geq 1}\left(A_{k} \cap X_{i}\right)\right)>0\right\} .
$$

Além disso, se $j \in J$ então $\mu\left(\bigcup_{k \geq 1}\left(A_{k} \cap X_{j}\right)\right)>0$, e portanto existe $k_{o} \geq 1$ tal que $\mu\left(A_{k_{o}} \cap X_{j}\right)>0$. Logo,

$$
j \in\left\{i \in I: \mu\left(A_{k_{o}} \cap X_{i}\right)>0\right\} \subset L \stackrel{\text { def }}{=} \bigcup_{k \geq 1}\left\{i \in I: \mu\left(A_{k} \cap X_{i}\right)>0\right\},
$$

ou seja, $J \subset L$. Portanto, como $L$ é enumerável, $J$ é enumerável.

Corolário 4.1.6. Seja $\left(X_{i}\right)_{i \in I}$ uma decomposição essencial para o espaço de medida $(X, \mathcal{A}, \mu)$. Se $A \in \mathcal{A}$ é $\sigma$-finito para $\mu$ então:

(a) podemos escrever $A=A_{1} \cup A_{0}$, com $A_{1}, A_{0} \in \mathcal{A}, A_{1} \cap A_{0}=\emptyset$, $A_{1}$ contido na união de uma subfamília enumerável de $\left(X_{i}\right)_{i \in I}$ e $\mu\left(A_{0}\right)=0$;

(b) $\mu(A)=\sum_{i \in I} \mu\left(A \cap X_{i}\right)$.

DemonstraçÃo. Seja $I^{\prime}=\left\{i \in I: \mu\left(A \cap X_{i}\right)>0\right\}$. Sejam

$$
A_{1}=\bigcup_{i \in I^{\prime}}\left(A \cap X_{i}\right) \quad \text { e } \quad A_{0}=A \backslash A_{1} .
$$

Claramente temos $A=A_{1} \cup A_{0}$ e $A_{1} \cap A_{0}=\emptyset$. Pelo Lema 4.1.5 I' é enumerável, logo $A_{1}, A_{0} \in \mathcal{A}$ e $\left(X_{i}\right)_{i \in I^{\prime}}$ é uma subfamília enumerável de $\left(X_{i}\right)_{i \in I}$ cuja união contém $A_{1}$. Para finalizar a prova do item (a) resta mostrar que 
$\mu\left(A_{0}\right)=0$. Afirmamos que $\mu\left(A_{0} \cap X_{i}\right)=0$ para todo $i \in I$. Com efeito, para $i \in I^{\prime}$ o conjunto $A_{0} \cap X_{i}$ é vazio, pois

$$
A_{0}=A \backslash A_{1}=A \backslash\left(\bigcup_{i \in I^{\prime}}\left(A \cap X_{i}\right)\right)=A \backslash\left(\left(\bigcup_{i \in I^{\prime}} X_{i}\right) \cap A\right)=A \backslash \bigcup_{i \in I^{\prime}} X_{i} ;
$$

para $i \in I \backslash I^{\prime}$ o conjunto $A_{0} \cap X_{i}$ está contido em $A \cap X_{i}$, e portanto tem medida nula. Seja $B$ um subconjunto mensurável de $A_{0}$ de medida finita. Como $\mu\left(B \cap X_{i}\right) \leq \mu\left(A_{0} \cap X_{i}\right)=0$ para todo $i \in I$, e $\left(X_{i}\right)_{i \in I}$ é uma decomposição essencial para $(X, \mathcal{A}, \mu)$, temos $\mu(B)=0$. Uma vez que $A$ é $\sigma$-finito para $\mu, A_{0}$ também é, logo existe uma seqüência $\left(B_{n}\right)_{n \geq 1}$ de subconjuntos mensuráveis de $X \operatorname{com} \mu\left(B_{n}\right)<+\infty$ tal que $A_{0}=\bigcup_{n \geq 1} B_{n}$. Assim, $\mu\left(A_{0}\right)=\mu\left(\bigcup_{n \geq 1} B_{n}\right) \leq \sum_{n \geq 1} \mu\left(B_{n}\right)=0$, i.e., $\mu\left(A_{0}\right)=0$. Para provar o item (b), observamos que $A$ é a união disjunta de $A_{0}$ e $A_{1}$, e que $\left(A \cap X_{i}\right)_{i \in I^{\prime}}$ é uma família enumerável de subconjuntos mensuráveis de $X$ essencialmente disjunta. Então, pelo Lema 4.1.2, temos:

$$
\mu(A)=\mu\left(A_{1}\right)=\mu\left(\bigcup_{i \in I^{\prime}}\left(A \cap X_{i}\right)\right)=\sum_{i \in I^{\prime}} \mu\left(A \cap X_{i}\right)=\sum_{i \in I} \mu\left(A \cap X_{i}\right) .
$$

Proposição 4.1.7. Se $\left(X_{i}\right)_{i \in I}$ é uma decomposição essencial para o espaço de medida $(X, \mathcal{A}, \mu)$, então:

(a) $\mu_{\mathrm{lb}}(A)=\sum_{i \in I} \mu\left(A \cap X_{i}\right)$, para todo $A \in \mathcal{A}$;

(b) se $\mu$ é cheia e completa então $A \subset X$ é mensurável se e somente se $A \cap X_{i}$ é mensurável para todo $i \in I$.

\section{DEMONSTRAÇÃO.}

- Prova de (a).

Se $A \in \mathcal{A}$ então, pelo item (a) da Proposição 2.1.7, $A$ admite um subconjunto $E, \sigma$-finito para $\mu$, tal que $\mu_{\mathrm{lb}}(A)=\mu(E)$. Portanto, pelo item (b) do Corolário 4.1.6 temos que $\mu_{\mathrm{lb}}(A)=\mu(E)=\sum_{i \in I} \mu\left(E \cap X_{i}\right)$. Se $\mu_{\mathrm{lb}}(A)=+\infty$ então

$$
\sum_{i \in I} \mu\left(A \cap X_{i}\right) \geq \sum_{i \in I} \mu\left(E \cap X_{i}\right)=+\infty
$$

i.e., $\mu_{\mathrm{lb}}(A)=+\infty=\sum_{i \in I} \mu\left(A \cap X_{i}\right)$. Suponhamos agora $\mu_{\mathrm{lb}}(A)<+\infty$. De $A=E \cup(A \backslash E)$ segue que $\mu_{\mathrm{lb}}(A)=\mu_{\mathrm{lb}}(E)+\mu_{\mathrm{lb}}(A \backslash E)$. Uma vez que $\mu_{\mathrm{lb}}(A)<+\infty$ e $\mu_{\mathrm{lb}}(E)=\mu(E)$ (item (b) da Proposição 2.1.7), 
temos $\mu_{\mathrm{lb}}(A \backslash E)=0$. Observe que, para cada $i \in I$,

$$
\mu\left((A \backslash E) \cap X_{i}\right) \leq \mu\left(X_{i}\right)<+\infty,
$$

e conseqüentemente $(A \backslash E) \cap X_{i}$ é $\sigma$-finito para $\mu$; portanto

$$
\mu\left((A \backslash E) \cap X_{i}\right)=\mu_{\mathrm{lb}}\left((A \backslash E) \cap X_{i}\right) \leq \mu_{\mathrm{lb}}(A \backslash E)=0,
$$

i.e., $\mu\left((A \backslash E) \cap X_{i}\right)=0$. Assim, para cada $i \in I$,

$$
\begin{array}{r}
\mu\left(A \cap X_{i}\right)=\mu\left((E \cup(A \backslash E)) \cap X_{i}\right)=\mu\left(\left(E \cap X_{i}\right) \cup\left((A \backslash E) \cap X_{i}\right)\right) \\
=\mu\left(E \cap X_{i}\right)+\mu\left((A \backslash E) \cap X_{i}\right)=\mu\left(E \cap X_{i}\right) .
\end{array}
$$

E portanto, $\mu_{\mathrm{lb}}(A)=\sum_{i \in I} \mu\left(E \cap X_{i}\right)=\sum_{i \in I} \mu\left(A \cap X_{i}\right)$.

- Prova de (b).

Se $A \subset X$ é mensurável então é claro que $A \cap X_{i}$ é mensurável para todo $i \in I$. Reciprocamente, suponhamos que $A \cap X_{i}$ seja mensurável para todo $i \in I$. Uma vez que $\mu$ é cheia, afim de provar a mensurabilidade de $A$ é suficiente mostrar que $A \cap E$ é mensurável para cada $E \in \mathcal{A} \operatorname{com} \mu(E)<+\infty$. Se $E \in \mathcal{A}$ e $\mu(E)<+\infty$ então $E$ é $\sigma$-finito para $\mu$ e assim, pelo item (a) do Corolário 4.1.6, existem $E_{1}, E_{0} \in \mathcal{A}$ com $E=E_{1} \cup E_{0}, E_{1} \cap E_{0}=\emptyset, E_{1} \subset \bigcup_{i \in I^{\prime}} X_{i}$ para algum subconjunto enumerável $I^{\prime} \subset I$ e $\mu\left(E_{0}\right)=0$. Desse modo,

$$
A \cap E_{1}=\left(A \cap E_{1}\right) \cap\left(\bigcup_{i \in I^{\prime}} X_{i}\right)=\bigcup_{i \in I^{\prime}}\left(\left(A \cap X_{i}\right) \cap E_{1}\right),
$$

o que mostra que $A \cap E_{1}$ é mensurável (pois $E_{1} \in \mathcal{A}$ e $A \cap X_{i} \in \mathcal{A}$ para todo $i \in I$ ). Além disso, uma vez que $\mu$ é completa e $\mu\left(E_{0}\right)=0$, o conjunto $A \cap E_{0}$ também é $\mathcal{A}$-mensurável. Portanto,

$$
A \cap E=A \cap\left(E_{1} \cup E_{0}\right)=\left(A \cap E_{1}\right) \cup\left(A \cap E_{0}\right) \in \mathcal{A} .
$$

Proposição 4.1.8. Todo espaço de medida $(X, \mathcal{A}, \mu)$ admite uma decomposição essencial. Além disso, qualquer família $\left(X_{i}\right)_{i \in I}$ essencialmente disjunta, de subconjuntos mensuráveis de $X$ de medida positiva e finita pode ser estendida para uma decomposição essencial para $(X, \mathcal{A}, \mu)$.

Demonstração. Denotemos por $\Omega$ a coleção de todos os subconjuntos $\mathcal{C}$ de $\mathcal{A}$ tais que: 
106 4. DECOMPOSIÇÃO DE UM ESPAÇO DE MEDIDA E O TEOREMA DE RIESZ

- $0<\mu(A)<+\infty$, para todo $A \in \mathcal{C}$;

- $\mu\left(A_{1} \cap A_{2}\right)=0$, para todo $A_{1}, A_{2} \in \mathcal{C} \operatorname{com} A_{1} \neq A_{2}$.

Se $\Omega$ é parcialmente ordenado pela relação de inclusão então claramente toda cadeia em $\Omega$ tem um limite superior (se $\mathcal{B} \in \Omega$ é uma cadeia então $\bigcup_{B \in \mathcal{B}} B$ é um limite superior para $\mathcal{B}$ e pertence a $\Omega$ ). Portanto, pelo Lema de Zorn, $\Omega$ admite um elemento maximal $\mathcal{C}$. Observe que se $A \in \mathcal{A}, \mu(A)<+\infty$ e $\mu\left(A \cap A^{\prime}\right)=0$ para todo $A^{\prime} \in \mathcal{C}$ então $\mu(A)=0$, do contrário $\mathcal{C} \cup\{A\}$ seria um elemento de $\Omega$ contendo propriamente $\mathcal{C}$, contradizendo a maximalidade de $\mathcal{C}$. Segue que os elementos de $\mathcal{C}$ constituem uma decomposição essencial para $(X, \mathcal{A}, \mu)$. Para finalizar, observamos que se $\left(X_{i}\right)_{i \in I}$ é uma família essencialmente disjunta de subconjuntos mensuráveis de $X$ de medida positiva finita, então o conjunto $\mathcal{C}_{0}=\left\{X_{i}: i \in I\right\}$ está em $\Omega$. Logo, considerando a coleção de todos os elementos de $\Omega$ que contêm $\mathcal{C}_{0}$, e usando novamente o Lema de Zorn, podemos obter um elemento maximal $\mathcal{C}$ de $\Omega$ que contém o conjunto $\mathcal{C}_{0}$.

LEMA 4.1.9. Um espaço medida $(X, \mathcal{A}, \mu)$ admite uma decomposição essencial $\left(X_{i}\right)_{i \in I}$ finita (i.e., I é finito) se e somente se $\mu_{\mathrm{lb}}(X)<+\infty$.

DemonstraçÃo. Se $\left(X_{i}\right)_{i \in I}$ é uma decomposição essencial para o espaço $(X, \mathcal{A}, \mu)$ com $I$ finito então, pelo item (a) do Lema 4.1.7, temos

$$
\mu_{\mathrm{lb}}(X)=\sum_{i \in I} \mu\left(X \cap X_{i}\right)=\sum_{i \in I} \mu\left(X_{i}\right)<+\infty,
$$

pois $\mu\left(X_{i}\right)<+\infty$ para cada $i \in I$. Reciprocamente, se $\mu_{\mathrm{lb}}(X)<+\infty$ então $X$ é $\sigma$-finito para $\mu_{\mathrm{lb}}$, e pelo item (d) da Proposição 2.1.7, podemos escrever $X=X_{0} \cup X_{\infty}$, com $X_{0}, X_{\infty} \in \mathcal{A}, X_{0} \cap X_{\infty}=\emptyset, X_{0} \sigma$-finito para $\mu$ e $\mu_{\mathrm{lb}}\left(X_{\infty}\right)=0$. Observe que $\mu\left(X_{0}\right)=\mu_{\mathrm{lb}}\left(X_{0}\right)=\mu_{\mathrm{lb}}(X)-\mu_{\mathrm{lb}}\left(X_{\infty}\right)=\mu_{\mathrm{lb}}(X)$. Se $\mu_{\mathrm{lb}}(X)=0$ então $\mu(X)=0$ ou $X$ é um bloco infinito para $\mu$; logo, cada subconjunto mensurável de $X$ de medida $\mu$ finita tem medida nula, e portanto a família vazia é uma decomposição essencial para $(X, \mathcal{A}, \mu)$. Por outro lado, se $\mu_{\mathrm{lb}}(X)>0$ então a família unitária consistindo apenas do conjunto $X_{0}$ é uma decomposição essencial para o espaço $(X, \mathcal{A}, \mu)$. Com efeito, seja $A \in \mathcal{A} \operatorname{com} \mu(A)<+\infty$ e $\mu\left(A \cap X_{0}\right)=0 ; \operatorname{como} \mu_{\mathrm{lb}}\left(X_{\infty}\right)=0$ temos que $\mu\left(X_{\infty}\right)=0$ ou $X_{\infty}$ é um bloco infinito para $\mu$. Se $\mu\left(X_{\infty}\right)=0$ então obviamente $\mu\left(A \cap X_{\infty}\right)=0$, e se $X_{\infty}$ é um bloco infinito para $\mu$ 
então também temos $\mu\left(A \cap X_{\infty}\right)=0$, pois $\mu\left(A \cap X_{\infty}\right) \leq \mu(A)<+\infty$. Assim, uma vez que $A=\left(A \cap X_{0}\right) \cup\left(A \cap X_{\infty}\right)$ e $X_{0} \cap X_{\infty}=\emptyset$, segue que $\mu(A)=\mu\left(A \cap X_{0}\right)+\mu\left(A \cap X_{\infty}\right)=0$.

Proposição 4.1.10. Se $\mu_{\mathrm{lb}}(X)=+\infty$ então, para quaisquer duas decomposições essenciais $\left(X_{i}\right)_{i \in I}$ e $\left(Y_{j}\right)_{j \in J}$ para o espaço de medida $(X, \mathcal{A}, \mu)$, temos $|I|=|J|$ (i.e., os conjuntos $I$ e $J$ têm a mesma cardinalidade).

Demonstração. Observe que, pelo Lema 4.1.9, os conjuntos $I$ e $J$ são ambos infinitos. Para cada $j \in J$ seja $I_{j}$ o conjunto

$$
I_{j}=\left\{i \in I: \mu\left(Y_{j} \cap X_{i}\right)>0\right\} .
$$

Pelo Lema 4.1 .5 o conjunto $I_{j}$ é enumerável. Sendo $\left(X_{i}\right)_{i \in I}$ uma decomposição essencial para $(X, \mathcal{A}, \mu)$, para cada $i \in I$ temos $0<\mu\left(X_{i}\right)<+\infty$, e portanto $X_{i}$ é $\sigma$-finito para $\mu$. Daí, pelo item (b) do Corolário 4.1.6, temos que $0<\mu\left(X_{i}\right)=\sum_{j \in J} \mu\left(X_{i} \cap Y_{j}\right)$ para cada $i \in I$; isso significa que para todo $i \in I$ existe $j \in J$ com $\mu\left(Y_{j} \cap X_{i}\right)>0$, i.e., $i \in I_{j}$. Assim, temos $I=\bigcup_{j \in J} I_{j}$, o que implica $|I| \leq|J| \cdot \aleph_{0}=|J|$. Analogamente concluímos que $|J| \leq|I|$, e conseqüentemente $|I|=|J|$.

DefiniçÃo 4.1.11. Sejam $(X, \mathcal{A}, \mu)$ um espaço de medida e $\left(X_{i}\right)_{i \in I}$ uma decomposição essencial arbitrária para $(X, \mathcal{A}, \mu)$. A dimensão $\operatorname{dim}(X, \mathcal{A}, \mu)$ do espaço $(X, \mathcal{A}, \mu)$ é definida por:

$$
\operatorname{dim}(X, \mathcal{A}, \mu)= \begin{cases}0 & \text { se } \mu_{\mathrm{lb}}(X)=0 \\ 1 & \text { se } 0<\mu_{\mathrm{lb}}(X)<+\infty \\ |I| & \text { se } \mu_{\mathrm{lb}}(X)=+\infty\end{cases}
$$

Lema 4.1.12. Para qualquer espaço de medida $(X, \mathcal{A}, \mu)$, temos que $\operatorname{dim}(X, \mathcal{A}, \mu) \leq \aleph_{0}$ se e somente se $X$ é $\sigma$-finito para $\mu_{\mathrm{lb}}$.

DemonstraÇão. Suponhamos que $\operatorname{dim}(X, \mathcal{A}, \mu) \leq \aleph_{0} . \operatorname{Se} \operatorname{dim}(X, \mathcal{A}, \mu)$ é menor do que $\aleph_{0}$ então $\mu_{\mathrm{lb}}(X)<+\infty$, e portanto $X$ é $\sigma$-finito para $\mu_{\mathrm{lb}}$. $\operatorname{Se} \operatorname{dim}(X, \mathcal{A}, \mu)=\aleph_{0}$ então existe uma decomposição essencial $\left(X_{i}\right)_{i \in I}$ para o espaço $(X, \mathcal{A}, \mu)$ com $I$ infinito e enumerável. Para cada $i \in I$ temos $\mu_{\mathrm{lb}}\left(X_{i}\right)=\mu\left(X_{i}\right)<+\infty$, e pelo item (a) da Proposição 4.1.7 nós obtemos 
108 4. DECOMPOSIÇÃO DE UM ESPAÇO DE MEDIDA E O TEOREMA DE RIESZ

$\mu_{\mathrm{lb}}\left(X \backslash \bigcup_{i \in I} X_{i}\right)=0$. De fato, sendo $I$ enumerável temos $X \backslash \bigcup_{i \in I} X_{i} \in \mathcal{A}$ e $\mu_{\mathrm{lb}}\left(X \backslash \bigcup_{i \in I} X_{i}\right)=\sum_{j \in I} \mu\left(\left(X \backslash \bigcup_{i \in I} X_{i}\right) \cap X_{j}\right)=\sum_{j \in I} \mu\left(X_{j} \backslash \bigcup_{i \in I} X_{i}\right)=\sum_{j \in I} \mu(\emptyset)=0$.

Assim, como $X=\left(\bigcup_{i \in I} X_{i}\right) \cup\left(X \backslash \bigcup_{i \in I} X_{i}\right)$, segue que $X$ é $\sigma$-finito para $\mu_{\mathrm{lb}}$. Reciprocamente, suponhamos que $X$ seja $\sigma$-finito para $\mu_{\mathrm{lb}}$. Se $\mu_{\mathrm{lb}}<+\infty$ então a dimensão de $(X, \mathcal{A}, \mu)$ é finita. Suponhamos que $\mu_{\mathrm{lb}}=+\infty$. Sendo $X \sigma$-finito para $\mu_{\mathrm{lb}}$, pelo item (d) da Proposição 2.1.7, existem $X_{0}, X_{\infty} \in \mathcal{A}$ disjuntos, com $X_{0} \sigma$-finito para $\mu, \mu_{\mathrm{lb}}\left(X_{\infty}\right)=0$ e $X=X_{0} \cup X_{\infty}$. Uma vez que $X_{0}$ é $\sigma$-finito para $\mu$ e $\mu\left(X_{0}\right)=\mu_{\mathrm{lb}}\left(X_{0}\right)=\mu_{\mathrm{lb}}(X)-\mu_{\mathrm{lb}}\left(X_{\infty}\right)=+\infty$, podemos escrever $X_{0}=\bigcup_{i \in I} X_{i}$, em que $\left(X_{i}\right)_{i \in I}$ é uma família enumerável de subconjuntos mensuráveis de medida positiva finita, dois a dois disjuntos. Afirmamos que $\left(X_{i}\right)_{i \in I}$ é uma decomposição essencial para $(X, \mathcal{A}, \mu)$. Com efeito, seja $A \in \mathcal{A}$ com $\mu(A)<+\infty$ e $\mu\left(A \cap X_{i}\right)=0$ para todo $i \in I$. Como $\mu\left(A \cap X_{\infty}\right) \leq \mu(A)<+\infty$, o conjunto $A \cap X_{\infty}$ é $\sigma$-finito para $\mu$, e então $\mu\left(A \cap X_{\infty}\right)=\mu_{\mathrm{lb}}\left(A \cap X_{\infty}\right) \leq \mu_{\mathrm{lb}}\left(X_{\infty}\right)=0$, i.e., $\mu\left(A \cap X_{\infty}\right)=0$. Assim,

$$
\begin{aligned}
& \mu(A)=\mu(A\left.\cap\left(X_{0} \cup X_{\infty}\right)\right)=\mu\left(A \cap X_{0}\right)+\mu\left(A \cap X_{\infty}\right) \\
&=\mu\left(A \cap\left(\bigcup_{i \in I} X_{i}\right)\right)=\mu\left(\bigcup_{i \in I}\left(A \cap X_{i}\right)\right)=\sum_{i \in I} \mu\left(A \cap X_{i}\right)=0 .
\end{aligned}
$$

Portanto $\operatorname{dim}(X, \mathcal{A}, \mu)=\aleph_{0}$.

DefiniÇÃo 4.1.13. Seja $\left(X_{i}\right)_{i \in I}$ uma decomposição essencial para o espaço de medida $(X, \mathcal{A}, \mu)$. Uma família $\left(X_{i}^{\prime}\right)_{i \in I}$ de subconjuntos mensuráveis de $X, \operatorname{com} X_{i}^{\prime} \subset X_{i}$ e $\mu\left(X_{i} \backslash X_{i}^{\prime}\right)=0$ para todo $i \in I$, é chamada de um refinamento da decomposição essencial $\left(X_{i}\right)_{i \in I}$.

OBSERVAÇÃo 4.1.14. Um refinamento $\left(X_{i}^{\prime}\right)_{i \in I}$ de uma decomposição essencial $\left(X_{i}\right)_{i \in I}$ para um espaço de medida $(X, \mathcal{A}, \mu)$ também é uma decomposição essencial para $(X, \mathcal{A}, \mu)$. Além disso, se $\left(X_{i}^{\prime \prime}\right)_{i \in I}$ é um refinamento de $\left(X_{i}^{\prime}\right)_{i \in I}$ então é um refinamento de $\left(X_{i}\right)_{i \in I}$. De fato, se $i, j \in I$ com $i \neq j$, então $\mu\left(X_{i}^{\prime} \cap X_{j}^{\prime}\right) \leq \mu\left(X_{i} \cap X_{j}\right)=0$, i.e., $\mu\left(X_{i}^{\prime} \cap X_{j}^{\prime}\right)=0$. Note que, para cada $i \in I, X_{i}$ é a união disjunta de $X_{i}^{\prime}$ e $X_{i} \backslash X_{i}^{\prime}$, logo $\mu\left(X_{i}\right)=\mu\left(X_{i}^{\prime}\right)+\mu\left(X_{i} \backslash X_{i}^{\prime}\right)=\mu\left(X_{i}^{\prime}\right)$. Portanto $0<\mu\left(X_{i}^{\prime}\right)<+\infty$ para cada $i \in I$. Agora, seja $A \in \mathcal{A} \operatorname{com} \mu(A)<+\infty$ e $\mu\left(A \cap X_{i}^{\prime}\right)=0$ para todo $i \in I$. Como $\mu\left(A \cap X_{i}\right)=\mu\left(A \cap X_{i}^{\prime}\right)+\mu\left(A \cap\left(X_{i} \backslash X_{i}^{\prime}\right)\right)$ e 
$\mu\left(A \cap\left(X_{i} \backslash X_{i}^{\prime}\right)\right) \leq \mu\left(X_{i} \backslash X_{i}^{\prime}\right)=0$, segue que $\mu\left(A \cap X_{i}\right)=\mu\left(A \cap X_{i}^{\prime}\right)$ para cada $i \in I$. Assim, uma vez que $\left(X_{i}\right)_{i \in I}$ é uma decomposição essencial para $(X, \mathcal{A}, \mu)$, temos $\mu(A)=0$, o que mostra que $\left(X_{i}^{\prime}\right)_{i \in I}$ também é uma decomposição essencial para $(X, \mathcal{A}, \mu)$. Por fim, para cada $i \in I$, temos $X_{i}^{\prime \prime} \subset X_{i}^{\prime} \subset X_{i}$ e $\mu\left(X_{i} \backslash X_{i}^{\prime \prime}\right)=\mu\left(X_{i} \backslash X_{i}^{\prime}\right)+\mu\left(X_{i}^{\prime} \backslash X_{i}^{\prime \prime}\right)=0$; portanto $\left(X_{i}^{\prime \prime}\right)_{i \in I}$ é um refinamento de $\left(X_{i}\right)_{i \in I}$.

LEMA 4.1.15. Se um espaço de medida $(X, \mathcal{A}, \mu)$ admite uma decomposição $\left(X_{i}\right)_{i \in I}$ então toda decomposição essencial $\left(Y_{j}\right)_{j \in J}$ para $(X, \mathcal{A}, \mu)$ pode ser refinada para uma decomposição para $(X, \mathcal{A}, \mu)$.

Demonstração. Para cada $j \in J$, seja $I_{j}$ o conjunto

$$
I_{j}=\left\{i \in I: \mu\left(Y_{j} \cap X_{i}\right)>0\right\}
$$

e para cada $i \in I$, seja $J_{i}$ o conjunto

$$
J_{i}=\left\{j \in J: \mu\left(Y_{j} \cap X_{i}\right)>0\right\} .
$$

Pelo Lema 4.1.5 os conjuntos $I_{j}$ e $J_{i}$ são enumeráveis para todo $j \in J$ e todo $i \in I$. Fixemos $j \in J$ e consideremos o conjunto:

$$
Y_{j}^{1}=\bigcup_{i \in I_{j}}\left(Y_{j} \cap X_{i}\right) \subset Y_{j}
$$

Argumentando como na demonstração do Corolário 4.1.6, concluímos que $\mu\left(Y_{j} \backslash Y_{j}^{1}\right)=0$ (com as notações do Corolário 4.1.6, para $A=Y_{j}, A_{1}=Y_{j}^{1} \mathrm{e}$ $A_{0}=Y_{j} \backslash Y_{j}^{1}$ temos $\left.\mu\left(A_{0}\right)=0\right)$. Portanto a família $\left(Y_{j}^{1}\right)_{j \in J}$ é um refinamento de $\left(Y_{j}\right)_{j \in J}$. Nosso objetivo agora é, a partir de $\left(Y_{j}^{1}\right)_{j \in J}$, obter um novo refinamento que seja uma decomposição para o espaço $(X, \mathcal{A}, \mu)$. Afirmamos que, para cada $j \in J$, temos

$$
\Omega_{j} \stackrel{\text { def }}{=}\left\{k \in J: Y_{k}^{1} \cap Y_{j}^{1} \neq \emptyset\right\} \subset \bigcup_{i \in I_{j}} J_{i}
$$

Com efeito, fixando $j \in J$, seja $k \in \Omega_{j}$. Então $Y_{k}^{1} \cap Y_{j}^{1} \neq \emptyset$. Uma vez que $Y_{k}^{1} \subset \bigcup_{i \in I_{k}} X_{i}, Y_{j}^{1} \subset \bigcup_{i \in I_{j}} X_{i}$ e os conjuntos $X_{i}$ são dois a dois disjuntos, existe $i \in I_{j} \cap I_{k}$. Portanto $i \in I_{j}$ e $k \in J_{i}$, ou seja, $k \in \bigcup_{i \in I_{j}} J_{i}$, e conseqüentemente $\Omega_{j} \subset \bigcup_{i \in I_{j}} J_{i}$. Como $J_{i}$ e $I_{j}$ são enumeráveis segue que 
110 4. DECOMPOSIÇÃO DE UM ESPAÇO DE MEDIDA E O TEOREMA DE RIESZ

$\Omega_{j}$ é enumerável. Se $Z_{j} \subset Y_{j}^{1}$ é o conjunto

$$
Z_{j}=\bigcup_{\substack{k \in J \\ k \neq j}}\left(Y_{k}^{1} \cap Y_{j}^{1}\right)
$$

então, para todo $j \in J$, temos:

$$
\mu\left(Z_{j}\right)=\mu\left(\bigcup_{\substack{k \in J \\ k \neq j}}\left(Y_{k}^{1} \cap Y_{j}^{1}\right)\right)=\mu\left(\bigcup_{\substack{k \in \Omega_{j} \\ k \neq j}}\left(Y_{k}^{1} \cap Y_{j}^{1}\right)\right)=\sum_{\substack{k \in \Omega_{j} \\ k \neq j}} \mu\left(Y_{k}^{1} \cap Y_{j}^{1}\right)=0
$$

uma vez que $\mu\left(Y_{k}^{1} \cap Y_{j}^{1}\right) \leq \mu\left(Y_{k} \cap Y_{j}\right)=0$ para todo $k \in \Omega_{j} \backslash\{j\}$. Fazendo $Y_{j}^{\prime}=Y_{j}^{1} \backslash Z_{j}$ temos $Y_{j}^{\prime} \subset Y_{j}^{1}$ e $\mu\left(Y_{j}^{1} \backslash Y_{j}^{\prime}\right)=\mu\left(Z_{j}\right)=0$, para cada $j \in J$. Assim, $\left(Y_{j}^{\prime}\right)_{j \in J}$ é um refinamento de $\left(Y_{j}^{1}\right)_{j \in J}$, e portanto um refinamento de $\left(Y_{j}\right)_{j \in J}$. Como $\left(Y_{j}\right)_{j \in J}$ é uma decomposição essencial para $(X, \mathcal{A}, \mu)$, $\left(Y_{j}^{\prime}\right)_{j \in J}$ também é uma decomposição essencial para $(X, \mathcal{A}, \mu)$. Observe que se $k, p \in J$ com $k \neq p$, então $Y_{k}^{1} \cap Y_{p}^{1} \subset Z_{p} ; \operatorname{logo}$

$$
Y_{p}^{\prime}=Y_{p}^{1} \backslash Z_{p} \subset Y_{p}^{1} \backslash\left(Y_{k}^{1} \cap Y_{p}^{1}\right)=Y_{p}^{1} \backslash Y_{k}^{1}
$$

Como $Y_{k}^{\prime} \subset Y_{k}^{1}$ temos $Y_{k}^{\prime} \cap Y_{p}^{\prime}=\emptyset$, ou seja, os conjuntos $Y_{j}^{\prime}$ são dois a dois disjuntos. Portanto a família $\left(Y_{j}^{\prime}\right)_{j \in J}$ é uma decomposição para $(X, \mathcal{A}, \mu)$ que refina a decomposição essencial $\left(Y_{j}\right)_{j \in J}$.

Proposição 4.1.16. Se $(X, \mathcal{A}, \mu)$ é um espaço de medida tal que $\operatorname{dim}(X, \mathcal{A}, \mu) \leq \aleph_{1}$ então $(X, \mathcal{A}, \mu)$ admite uma decomposição .

DemonstraÇão. Seja $\left(X_{i}\right)_{i \in I}$ uma decomposição essencial para o espaço $(X, \mathcal{A}, \mu)$. Uma vez que $|I| \leq \aleph_{1}$, podemos bem ordenar o conjunto de índices $I$ de modo que, para todo $i \in I$, o segmento inicial $\{j \in I: j<i\}$ seja enumerável. Para cada $i \in I$, seja

$$
Y_{i}=X_{i} \backslash \bigcup_{j<i}\left(X_{i} \cap X_{j}\right)
$$

Como $\bigcup_{j<i}\left(X_{i} \cap X_{j}\right) \subset X_{i}$, segue que

$$
\mu\left(X_{i} \backslash Y_{i}\right)=\mu\left(\bigcup_{j<i}\left(X_{i} \cap X_{j}\right)\right) \leq \sum_{j<i} \mu\left(X_{i} \cap X_{j}\right)=0 .
$$

Portanto $\left(Y_{i}\right)_{i \in I}$ é um refinamento de $\left(X_{i}\right)_{i \in I}$. Observe que os conjuntos $Y_{i}$ são dois a dois disjuntos. Com efeito, se $i, j$ são elementos de $I$ com $i \neq j$, digamos $i<j$, temos $Y_{i}=X_{i} \backslash \bigcup_{k<i}\left(X_{i} \cap X_{k}\right) \subset X_{i}$ e 


$$
Y_{j}=X_{j} \backslash \bigcup_{k<j}\left(X_{j} \cap X_{k}\right) \subset X_{j} \backslash\left(X_{j} \cap X_{i}\right)=X_{j} \backslash X_{i},
$$

o que mostra que $Y_{i} \cap Y_{j}=\emptyset$. Assim, pela Observação 4.1.14, a família $\left(Y_{i}\right)_{i \in I}$ é uma decomposição para o espaço $(X, \mathcal{A}, \mu)$.

Exemplo 4.1.17. Seja $(X, \mathcal{A}, \mu)$ o espaço de medida perfeita contruído no Exemplo 2.4.1. Seja $\mathcal{F}$ a família formada por todas as linhas e todas as colunas de $X$, ou seja,

$$
\mathcal{F}:\left(\{x\} \times C_{2}\right)_{x \in C_{1}},\left(C_{1} \times\{y\}\right)_{y \in C_{2}} .
$$

Observe que duas linhas distintas de $X$ são disjuntas e duas colunas distintas de $X$ também são disjuntas; além disso, para cada $x \in C_{1}$ e $y \in C_{2}$ temos

$$
\left(\{x\} \times C_{2}\right) \cap\left(C_{1} \times\{y\}\right)=\{(x, y)\} \stackrel{\text { def }}{=} B .
$$

Como a $x$-ésima coluna de $B$ igual a $\{y\}$, a $y$-ésima linha de $B$ é igual a $\{x\}$, e as demais linhas e colunas de $B$ são vazias, segue que $\mu(B)=0$, e portanto $\mathcal{F}$ é uma família essencialmente disjunta de $X$. Afirmamos que $\mathcal{F}$ é uma decomposição essencial para $X$. De fato, para cada $x \in C_{1}$ temos

$$
\left(\{x\} \times C_{2}\right)_{x^{\prime}}= \begin{cases}C_{2} & \text { se } x^{\prime}=x \\ \emptyset & \text { se } x^{\prime} \neq x\end{cases}
$$

e portanto $\mu\left(\{x\} \times C_{2}\right)=1$. Analogamente temos $\mu\left(C_{1} \times\{y\}\right)=1$ para cada $y \in C_{2}$. Assim, $0<\mu(A)<+\infty$ para todo $A \in \mathcal{F}$. Seja agora $E \in \mathcal{A}$, $\mu(E)<+\infty$, tal que $\mu(E \cap A)=0$ para todo $A \in \mathcal{F}$. Fixemos $x \in C_{1}$. Se $\mu\left(E \cap\left(\{x\} \times C_{2}\right)\right)=0$ então $\mu_{x}\left(E \cap\left(\{x\} \times C_{2}\right)\right)=0$; logo a $x$-ésima coluna de $E \cap\left(\{x\} \times C_{2}\right)$ é enumerável. Como a $x$-ésima coluna de $E \cap\left(\{x\} \times C_{2}\right)$ e a $x$-ésima coluna de $E$ são iguais, segue que $E_{x}$ é enumerável, e portanto $\mu_{x}(E)=0$. De modo análogo temos $\mu^{y}(E)=0$ para cada $y \in C_{2}$. Assim,

$$
\mu(E)=\sum_{x \in C_{1}} \mu_{x}(E)+\sum_{y \in C_{2}} \mu^{y}(E)=0,
$$

o que mostra que $\mathcal{F}$ é uma decomposição essencial para $(X, \mathcal{A}, \mu)$. Desse modo, concluímos que $\operatorname{dim}(X, \mathcal{A}, \mu)=\left|C_{1}\right|+\left|C_{2}\right|=\max \left\{\left|C_{1}\right|,\left|C_{2}\right|\right\}$. Suponhamos que $\left|C_{1}\right|=\left|C_{2}\right|=\aleph_{1}$. Então $\operatorname{dim}(X, \mathcal{A}, \mu)=\aleph_{1}$, e portanto, pela Proposição 4.1.16, o espaço $(X, \mathcal{A}, \mu)$ admite uma decomposição. Vamos exibir uma tal decomposição. Se $\left|C_{1}\right|=\left|C_{2}\right|=\aleph_{1}$ então, pela Proposição 2.4.4, 
existe um subconjunto $R \subset X$ tal que $C_{2} \backslash R_{x}$ e $R^{y}$ são enumeráveis para todo $x \in C_{1}$ e todo $y \in C_{2}$. Afirmamos que a família

$$
\mathcal{G}:\left(\{x\} \times R_{x}\right)_{x \in C_{1}},\left(\left(C_{1} \backslash R^{y}\right) \times\{y\}\right)_{y \in C_{2}}
$$

é uma decomposição para $X$. Observe que para cada $x \in C_{1}$ e $y \in C_{2}$ temos

$$
\{x\} \times R_{x} \subset\{x\} \times C_{2} \quad \text { e } \quad\left(C_{1} \backslash R^{y}\right) \times\{y\} \subset C_{1} \times\{y\} .
$$

Além disso, $\mu\left(\left(\{x\} \times C_{2}\right) \backslash\left(\{x\} \times R_{x}\right)\right)=\mu\left(\{x\} \times\left(C_{2} \backslash R_{x}\right)\right)=0$, pois $\{x\} \times\left(C_{2} \backslash R_{x}\right)$ é enumerável, e conseqüentemente são enumeráveis todas as suas linhas e colunas. Do mesmo modo $\left(C_{1} \times\{y\}\right) \backslash\left(\left(C_{1} \backslash R^{y}\right) \times\{y\}\right)$ tem medida nula, e isso mostra que $\mathcal{G}$ é um refinamento de $\mathcal{F}$. Assim, pela Observação 4.1.14, $\mathcal{G}$ é uma decomposição essencial para $X$. Por fim, se $(x, y) \in\{x\} \times R_{x}$ então $y \in R_{x}$, o que implica $x \in R^{y} ; \operatorname{logo}(x, y)$ não está em $\left(C_{1} \backslash R^{y}\right) \times\{y\}$, mostrando que os elementos de $\mathcal{G}$ são dois a dois disjuntos. Portanto $\mathcal{G}$ é, de fato, uma decomposição para $(X, \mathcal{A}, \mu)$. Veremos na próxima seção resultados (Teorema 4.2.7 e Corolário 4.2.8) que nos permitirão concluir que a aplicação de Riesz (1.4.4) de $(X, \mathcal{A}, \mu)$ é uma isometria linear.

\subsection{Generalizando o Teorema de Representação de Riesz}

Proposição 4.2.1. Sejam $\left(X_{i}, \mathcal{A}_{i}, \mu_{i}\right)_{i \in I}$ uma família de espaços de medida completa tal que $0<\mu_{i}\left(X_{i}\right)<+\infty$ para todo $i \in I$, e $\left(X^{\prime}, \mathcal{A}^{\prime}, \mu^{\prime}\right)$ a sua soma externa $\sum_{i \in I}\left(X_{i}, \mathcal{A}_{i}, \mu_{i}\right)$. Seja $\sim$ uma relação de equivalência em $X^{\prime}=\sum_{i \in I} X_{i}$ satisfazendo a seguinte propriedade:

(*) o conjunto $\left\{x \in X_{i}: x \sim y\right.$, para algum $\left.y \in X_{j}\right\}$ tem medida nula para todo $i, j \in I$ com $i \neq j$; e para todo $i \in I$ e todo $x, y \in X_{i}$ temos que $x \sim y$ se e somente se $x=y$.

Seja $X=\left(\sum_{i \in I} X_{i}\right) / \sim$ e sejam $\mathcal{A}$ e $\mu$ respectivamente a $\sigma$-álgebra e a medida co-induzidas em $X$ pela aplicação canônica $\mathfrak{q}: \sum_{i \in I} X_{i} \rightarrow X$. Então $(X, \mathcal{A}, \mu)$ é um espaço de medida perfeita, $\left(\mathfrak{q}\left(X_{i}\right)\right)_{i \in I}$ é uma decomposição essencial para $X$ e $\left.\mathfrak{q}\right|_{X_{i}}: X_{i} \rightarrow \mathfrak{q}\left(X_{i}\right)$ é um isomorfismo para todo $i \in I$. Além disso, para $p<+\infty$, a aplicação

$$
\Phi: L^{p}(X, \mathcal{A}, \mu) \ni f \longmapsto f \circ \mathfrak{q} \in L^{p}\left(\sum_{i \in I}\left(X_{i}, \mathcal{A}_{i}, \mu_{i}\right)\right),
$$

induzida por $\mathfrak{q}$ em $L^{p}$, é uma isometria linear. 
DemonstraÇÃo. Vamos dividir a demonstração em vários passos.

Passo 1. Para cada subconjunto $A \subset X$ temos que $A \in \mathcal{A}$ se e somente se $\mathfrak{q}^{-1}(A) \cap X_{i} \in \mathcal{A}_{i}$ para cada $i \in I$; nesse caso,

$$
\mu(A)=\sum_{i \in I} \mu_{i}\left(\mathfrak{q}^{-1}(A) \cap X_{i}\right) .
$$

Para cada $A \subset X, A \in \mathcal{A}=\mathfrak{q}_{*} \mathcal{A}^{\prime}$ se e somente se $\mathfrak{q}^{-1}(A) \in \mathcal{A}^{\prime}$, ou seja, se e somente se $\mathfrak{q}^{-1}(A) \cap X_{i} \in \mathcal{A}_{i}$ para todo $i \in I$. Além disso,

$$
\mu(A)=\left(\mathfrak{q}_{*} \mu^{\prime}\right)(A)=\mu^{\prime}\left(\mathfrak{q}^{-1}(A)\right)=\sum_{i \in I} \mu_{i}\left(\mathfrak{q}^{-1}(A) \cap X_{i}\right) .
$$

Passo 2. A medida $\mu$ é completa.

Sejam $A \in \mathcal{A}$ com $\mu(A)=0$ arbitrário e $B$ um subconjunto de $A$. Então, para cada $i \in I$ temos, pelo passo $1, \mathfrak{q}^{-1}(A) \cap X_{i} \in \mathcal{A}_{i}$ e

$$
\mu_{i}\left(\mathfrak{q}^{-1}(A) \cap X_{i}\right) \leq \sum_{i \in I} \mu_{i}\left(\mathfrak{q}^{-1}(A) \cap X_{i}\right)=\mu(A)=0,
$$

ou seja, $\mu_{i}\left(\mathfrak{q}^{-1}(A) \cap X_{i}\right)=0$. Assim, uma vez que para todo $i \in I$ $\mathfrak{q}^{-1}(B) \cap X_{i}$ é subconjunto de $\mathfrak{q}^{-1}(A) \cap X_{i}$ e $\mu_{i}$ é completa, segue que $\mathfrak{q}^{-1}(B) \cap X_{i} \in \mathcal{A}_{i}$ para todo $i \in I$; portanto $\mathfrak{q}^{-1}(B) \in \mathcal{A}^{\prime}$, i.e., $B \in \mathcal{A}$.

Passo 3. Para todo $i \in I$, um subconjunto $E \subset \mathfrak{q}\left(X_{i}\right)$ está em $\mathcal{A}$ se e somente se $\mathfrak{q}^{-1}(E) \cap X_{i} \in \mathcal{A}_{i}$; nesse caso $\mu(E)=\mu_{i}\left(\mathfrak{q}^{-1}(E) \cap X_{i}\right)$.

Pelo passo 1 , basta mostrar que para $j \neq i$ temos $\mathfrak{q}^{-1}(E) \cap X_{j} \in \mathcal{A}_{j}$ e $\mu_{j}\left(\mathfrak{q}^{-1}(E) \cap X_{j}\right)=0$. Observe que para $j \neq i$,

$$
\mathfrak{q}^{-1}(E) \cap X_{j} \subset \mathfrak{q}^{-1}\left(\mathfrak{q}\left(X_{i}\right)\right) \cap X_{j}=\mathcal{B}_{i},
$$

em que $\mathcal{B}_{i} \stackrel{\text { def }}{=}\left\{x \in X_{j}: x \sim y\right.$ para algum $\left.y \in X_{i}\right\}$. Pela propriedade (*) da relação de equivalência $\sim$, o conjunto $\mathcal{B}_{i}$ tem medida nula, e uma vez que $\mu_{j}$ é completa, temos $\mathfrak{q}^{-1}(E) \cap X_{j} \in \mathcal{A}_{j}$. Além disso,

$$
\mu_{j}\left(\mathfrak{q}^{-1}(E) \cap X_{j}\right) \leq \mu_{j}\left(\mathcal{B}_{i}\right)=0 \text {, i.e., } \mu_{j}\left(\mathfrak{q}^{-1}(E) \cap X_{j}\right)=0 .
$$

Passo 4. Para todo $i \in I, \mathfrak{q}\left(X_{i}\right) \in \mathcal{A}$ e $\left.\mathfrak{q}\right|_{X_{i}}: X_{i} \rightarrow \mathfrak{q}\left(X_{i}\right)$ é um isomorfismo.

Fixemos $i \in I$. Se $x, y \in X_{i}$ são tais que $\left.\mathfrak{q}\right|_{X_{i}}(x)=\left.\mathfrak{q}\right|_{X_{i}}(y)$ então $\mathfrak{q}(x)=\mathfrak{q}(y)$, ou seja $[x]=[y]$; portanto $x \sim y$, e pela propriedade $(*)$ 
temos $x=y$. Logo $\left.\mathfrak{q}\right|_{X_{i}}$ é injetora, e assim bijetora sobre sua imagem $\mathfrak{q}\left(X_{i}\right)$. Como $\mathfrak{q}^{-1}\left(\mathfrak{q}\left(X_{i}\right)\right) \supset X_{i}$, temos que $\mathfrak{q}^{-1}\left(\mathfrak{q}\left(X_{i}\right)\right) \cap X_{i}=X_{i} \subset \mathcal{A}_{i} ;$ logo, pelo passo $3, \mathfrak{q}\left(X_{i}\right) \in \mathcal{A}$. Para mostrar que $\left.\mathfrak{q}\right|_{X_{i}}: X_{i} \rightarrow \mathfrak{q}\left(X_{i}\right)$ é um isomorfismo, resta mostrar que $\left.\mathfrak{q}\right|_{X_{i}}$ é uma aplicação quociente. Observe que $\left.\mathfrak{q}\right|_{X_{i}}$ é a aplicação

$$
\left(X_{i}, \mathcal{A}_{i}, \mu_{i}\right) \ni x_{i} \longmapsto\left[x_{i}\right] \in\left(\mathfrak{q}\left(X_{i}\right), \mathcal{A}^{\prime \prime}, \mu^{\prime \prime}\right)
$$

em que $\mathcal{A}^{\prime \prime}=\left.\mathcal{A}\right|_{\mathfrak{q}\left(X_{i}\right)}$ e $\mu^{\prime \prime}=\left.\mu\right|_{\mathcal{A}^{\prime \prime}}$. A aplicação $\left.\mathfrak{q}\right|_{X_{i}}$ é uma aplicação quociente se $\left(\left.\mathfrak{q}\right|_{X_{i}}\right)_{*} \mathcal{A}_{i}=\mathcal{A}^{\prime \prime}$ e $\left(\left.\mathfrak{q}\right|_{X_{i}}\right)_{*} \mu_{i}=\mu^{\prime \prime}$. Com efeito,

$$
\begin{aligned}
& \left(\left.\mathfrak{q}\right|_{X_{i}}\right)_{*} \mathcal{A}_{i}=\left\{A \subset \mathfrak{q}\left(X_{i}\right):\left(\left.\mathfrak{q}\right|_{X_{i}}\right)^{-1}(A) \in \mathcal{A}_{i}\right\} \\
& \quad=\left\{A \subset \mathfrak{q}\left(X_{i}\right): \mathfrak{q}^{-1}(A) \cap X_{i} \in \mathcal{A}_{i}\right\}=\left\{A \subset \mathfrak{q}\left(X_{i}\right): A \in \mathcal{A}\right\}=\mathcal{A}^{\prime \prime}
\end{aligned}
$$

Se $E \subset \mathfrak{q}\left(X_{i}\right)$ e $E \in \mathcal{A}^{\prime \prime}=\mathcal{P}\left(\mathfrak{q}\left(X_{i}\right)\right) \cap \mathcal{A}$ então $E \in \mathcal{A}$ e portanto, pelo passo $3, \mathfrak{q}^{-1}(E) \cap X_{i} \in \mathcal{A}_{i}$. Assim,

$\mu^{\prime \prime}(E)=\mu(E)=\mu_{i}\left(\mathfrak{q}^{-1}(E) \cap X_{i}\right)=\mu_{i}\left(\left(\left.\mathfrak{q}\right|_{X_{i}}\right)^{-1}(E)\right)=\left(\left(\left.\mathfrak{q}\right|_{X_{i}}\right)_{*} \mu_{i}\right)(E)$,

o que mostra que $\mu^{\prime \prime}=\left(\left.\mathfrak{q}\right|_{X_{i}}\right)_{*} \mu_{i}$.

Passo 5. Para $A \subset X$, temos que $A \in \mathcal{A}$ se e somente se $A \cap \mathfrak{q}\left(X_{i}\right) \in \mathcal{A}$ para todo $i \in I$; nesse caso $\mu(A)=\sum_{i \in I} \mu\left(A \cap \mathfrak{q}\left(X_{i}\right)\right)$.

Seja $A$ um subconjunto de $X$. Pelo passo $1, A \in \mathcal{A}$ se e somente se $\mathfrak{q}^{-1}(A) \cap X_{i} \in \mathcal{A}_{i}$ para todo $i \in I$. Mas, para cada $i \in I$,

$$
\mathfrak{q}^{-1}\left(A \cap \mathfrak{q}\left(X_{i}\right)\right) \cap X_{i}=\mathfrak{q}^{-1}(A) \cap \mathfrak{q}^{-1}\left(\mathfrak{q}\left(X_{i}\right)\right) \cap X_{i}=\mathfrak{q}^{-1}(A) \cap X_{i},
$$

uma vez que $X_{i} \subset \mathfrak{q}^{-1}\left(\mathfrak{q}\left(X_{i}\right)\right)$. Assim, para cada $i \in I, \mathfrak{q}^{-1}(A) \cap X_{i} \in \mathcal{A}_{i}$ se e somente se $A \cap \mathfrak{q}\left(X_{i}\right) \in \mathcal{A}$ (passo 3 ), e portanto $A \in \mathcal{A}$ se e somente se $A \cap \mathfrak{q}\left(X_{i}\right) \in \mathcal{A}$ para todo $i \in I$. Ainda, pelo passo 3,

$$
\mu_{i}\left(\mathfrak{q}^{-1}(A) \cap X_{i}\right)=\mu_{i}\left(\mathfrak{q}^{-1}\left(A \cap \mathfrak{q}\left(X_{i}\right)\right) \cap X_{i}\right)=\mu\left(A \cap \mathfrak{q}\left(X_{i}\right)\right),
$$

para todo $i \in I$. Portanto (pelo passo 1),

$$
\mu(A)=\sum_{i \in I} \mu_{i}\left(\mathfrak{q}^{-1}(A) \cap X_{i}\right)=\sum_{i \in I} \mu\left(A \cap \mathfrak{q}\left(X_{i}\right)\right) .
$$

Passo 6. A família $\left(\mathfrak{q}\left(X_{i}\right)\right)_{i \in I}$ é uma decomposição essencial para $X$. 
Pelo passo $4, \mathfrak{q}\left(X_{i}\right) \in \mathcal{A}$ para todo $i \in I$. Sejam $i, j \in I$ com $i \neq j$. Como $\mathfrak{q}\left(X_{i}\right) \cap \mathfrak{q}\left(X_{j}\right) \subset \mathfrak{q}\left(X_{j}\right)$ e $\mathfrak{q}\left(X_{i}\right) \cap \mathfrak{q}\left(X_{j}\right) \in \mathcal{A}$, então, pelo passo 3,

$$
\begin{gathered}
\mathfrak{q}^{-1}\left(\mathfrak{q}\left(X_{i}\right)\right) \cap X_{j}=\mathfrak{q}^{-1}\left(\mathfrak{q}\left(X_{i}\right) \cap \mathfrak{q}\left(X_{j}\right)\right) \cap X_{j} \in \mathcal{A}_{j}, \quad \text { e } \\
\mu\left(\mathfrak{q}\left(X_{i}\right) \cap \mathfrak{q}\left(X_{j}\right)\right)=\mu_{j}\left(\mathfrak{q}^{-1}\left(\mathfrak{q}\left(X_{i}\right)\right) \cap X_{j}\right) .
\end{gathered}
$$

Portanto, pela propriedade $(*)$ da relação de equivalência $\sim$, temos que $\mu\left(\mathfrak{q}\left(X_{i}\right) \cap \mathfrak{q}\left(X_{j}\right)\right)=0$, o que mostra que $\left(\mathfrak{q}\left(X_{i}\right)\right)_{i \in I}$ é uma família essencialmente disjunta de $X$. Ainda pelo passo 3 ,

$$
\mu\left(\mathfrak{q}\left(X_{i}\right)\right)=\mu_{i}\left(\mathfrak{q}^{-1}\left(\mathfrak{q}\left(X_{i}\right)\right) \cap X_{i}\right)=\mu_{i}\left(X_{i}\right),
$$

e portanto $0<\mu\left(\mathfrak{q}\left(X_{i}\right)\right)<+\infty$ para todo $i \in I$. Além disso, se $A \in \mathcal{A}$ e $\mu\left(A \cap \mathfrak{q}\left(X_{i}\right)\right)=0$ para todo $i \in I$ então, pelo passo 5 ,

$$
\mu(A)=\sum_{i \in I} \mu\left(A \cap \mathfrak{q}\left(X_{i}\right)\right)=0
$$

e isso finaliza a prova de que a família $\left(\mathfrak{q}\left(X_{i}\right)\right)_{i \in I}$ é uma decomposição essencial para $X$.

Passo 7. A medida $\mu$ é cheia.

Seja $A \subset X$ tal que $A \cap E \in \mathcal{A}$ para todo $E \in \mathcal{A} \operatorname{com} \mu(E)<+\infty$. Uma vez que $\mathfrak{q}\left(X_{i}\right) \in \mathcal{A}$ e $\left.\mu\left(\mathfrak{q}\left(X_{i}\right)\right)\right)<+\infty$ para todo $i \in I$, segue que $A \cap \mathfrak{q}\left(X_{i}\right) \in \mathcal{A}$ para todo $i \in I$. Portanto, pelo passo $5, A \in \mathcal{A}$.

Passo 8. A medida $\mu$ é livre de blocos.

Seja $A \in \mathcal{A} \operatorname{com} \mu(A)=+\infty$. Pelo passo 5 temos que

$$
\mu(A)=\sum_{i \in I} \mu\left(A \cap \mathfrak{q}\left(X_{i}\right)\right)
$$

e portanto $\mu\left(A \cap \mathfrak{q}\left(X_{i}\right)\right)>0$ para algum $i \in I$. Por outro lado, pelo passo $6, \mu\left(A \cap \mathfrak{q}\left(X_{i}\right)\right) \leq \mu\left(\mathfrak{q}\left(X_{i}\right)\right)<+\infty$, provando que $A$ não é um bloco infinito para $\mu$.

Passo 9. Para $p<+\infty$, a aplicação $\Phi$ é uma isometria linear.

Pela Proposição 3.1.3, a soma externa $\left(X^{\prime}, \mathcal{A}^{\prime}, \mu^{\prime}\right)=\sum_{i \in I}\left(X_{i}, \mathcal{A}_{i}, \mu_{i}\right)$ é um espaço de medida completa. Como q é uma aplicação quociente, 
pelo Lema 1.4.86, a aplicação $\Phi$ é uma imersão isométrica e sua imagem é o conjunto

$\operatorname{Im}(\Phi)=\left\{g \in L^{p}\left(X^{\prime}, \mathcal{A}^{\prime}, \mu^{\prime}\right):\right.$ existe $f: X \rightarrow \mathbb{R}$ tal que $f \circ \mathfrak{q}=g \mu^{\prime}$-qs $\}$.

Logo, para provar que $\Phi$ é uma isometria linear é suficiente mostrar que para cada aplicação $g \in L^{p}\left(X^{\prime}, \mathcal{A}^{\prime}, \mu^{\prime}\right)$ existe uma aplicação $f: X \rightarrow \mathbb{R}$ tal que $f \circ \mathfrak{q}=g \mu^{\prime}$-qs. Se $g \in L^{p}\left(X^{\prime}, \mathcal{A}^{\prime}, \mu^{\prime}\right)$ então, pela Proposição 3.2.9, $\left(\left.g\right|_{X_{i}}\right)_{i \in I} \in \bar{\bigoplus}_{i \in I}^{p} L^{p}\left(X_{i}, \mathcal{A}_{i}, \mu_{i}\right)$. Visto que $p<+\infty$, a Observação 3.2 .5 garante que o conjunto $I^{\prime}=I \backslash\left\{i \in I:\left.g\right|_{X_{i}}=0 \mu_{i}\right.$-qs $\}$ é enumerável. Para cada $i \in I^{\prime}$, seja $Y_{i}$ o conjunto

$$
Y_{i}=X_{i} \backslash \bigcup_{\substack{j \in I^{\prime} \\ j \neq i}} \mathcal{B}_{j}
$$

no qual $\mathcal{B}_{j}=\left\{x \in X_{i}: x \sim y\right.$ para algum $\left.y \in X_{j}\right\}$. Para cada $j \in I^{\prime}$ com $j \neq i$ temos, pela propriedade $(*)$ da relação de equivalência $\sim$, que $\mu_{i}\left(\mathcal{B}_{j}\right)=0$. Assim,

$$
\mu_{i}\left(\bigcup_{\substack{j \in I^{\prime} \\ j \neq i}} \mathcal{B}_{j}\right) \leq \sum_{\substack{j \in I^{\prime} \\ j \neq i}} \mu_{i}\left(\mathcal{B}_{j}\right)=0
$$

e portanto $\mu_{i}\left(X_{i} \backslash Y_{i}\right)=0$ para todo $i \in I^{\prime}$. Além disso, os conjuntos $\mathfrak{q}\left(Y_{i}\right), i \in I^{\prime}$, são dois a dois disjuntos. Ora, se $i, j \in I^{\prime}$ e $i \neq j$ então

$$
Y_{i}=X_{i} \backslash \bigcup_{\substack{j \in I^{\prime} \\ j \neq i}} \mathcal{B}_{j} \subset X_{i} \backslash \mathcal{B}_{j} \quad \text { e } \quad \mathcal{B}_{j}=\left\{x \in X_{i}:[x] \in \mathfrak{q}\left(X_{j}\right)\right\} ;
$$

portanto $\mathfrak{q}\left(Y_{i}\right) \subset \mathfrak{q}\left(X_{i} \backslash \mathcal{B}_{j}\right)$ e $\mathfrak{q}\left(X_{i} \backslash \mathcal{B}_{j}\right) \cap \mathfrak{q}\left(X_{j}\right)=\emptyset ; \operatorname{como} \mathfrak{q}\left(Y_{j}\right) \subset \mathfrak{q}\left(X_{j}\right)$ segue que $\mathfrak{q}\left(Y_{i}\right) \cap \mathfrak{q}\left(Y_{j}\right)=\emptyset$. Considere a função $f: X \rightarrow \mathbb{R}$ tal que $\left.f \circ \mathfrak{q}\right|_{Y_{i}}=\left.g\right|_{Y_{i}}$ para todo $i \in I^{\prime}$ e tal que $f$ se anula fora do conjunto $\bigcup_{i \in I^{\prime}} \mathfrak{q}\left(Y_{i}\right)$. Vamos mostrar que $\left.f \circ \mathfrak{q}\right|_{X_{i}}=\left.g\right|_{X_{i}} \mu_{i}$-qs para todo $i \in I$, e daí seguirá que $f \circ \mathfrak{q}=g \mu^{\prime}$-qs. Com efeito, se $i \in I^{\prime}$ então $\left.f \circ \mathfrak{q}\right|_{X_{i}}=\left.g\right|_{X_{i}}$ em $Y_{i} \subset X_{i}$ e $\mu_{i}\left(X_{i} \backslash Y_{i}\right)=0$, logo $\left.f \circ \mathfrak{q}\right|_{X_{i}}=\left.g\right|_{X_{i}} \mu_{i}$-qs. Sejam $i \in I \backslash I^{\prime}$ fixado e $x \in X_{i} \backslash \bigcup_{j \in I^{\prime}} \mathcal{B}_{j}$ arbitrário. Então $x \notin \mathcal{B}_{j}$ para todo $j \in I^{\prime}$, ou seja, $\mathfrak{q}(x)=[x] \notin \mathfrak{q}\left(X_{j}\right)$ para todo $j \in I^{\prime}$; portanto $\mathfrak{q}(x) \notin \bigcup_{j \in I^{\prime}} \mathfrak{q}\left(X_{j}\right)$, e uma vez que $\bigcup_{j \in I^{\prime}} \mathfrak{q}\left(Y_{j}\right) \subset \bigcup_{j \in I^{\prime}} \mathfrak{q}\left(X_{j}\right)$ e $f$ se anula fora de $\bigcup_{j \in I^{\prime}} \mathfrak{q}\left(Y_{j}\right)$, temos $\left(\left.f \circ \mathfrak{q}\right|_{X_{i}}\right)(x)=(f \circ \mathfrak{q})(x)=f(\mathfrak{q}(x))=0$ para todo $x \in X_{i} \backslash \bigcup_{j \in I^{\prime}} \mathcal{B}_{j}$. Note que $\mu_{i}\left(\mathcal{B}_{j}\right)=0$ para cada $i \in I \backslash I^{\prime}$ 
e para todo $j \in I^{\prime}$, o que implica $\mu_{i}\left(\bigcup_{j \in I^{\prime}} \mathcal{B}_{j}\right) \leq \sum_{j \in I^{\prime}} \mu_{i}\left(\mathcal{B}_{j}\right)=0$, e conseqüentemente $\left.f \circ \mathfrak{q}\right|_{X_{i}}=0 \mu_{i}$-qs. Por outro lado, para cada $i \in I \backslash I^{\prime}$ temos $\left.g\right|_{X_{i}}=0 \mu_{i}$-qs, e assim $\left.f \circ \mathfrak{q}\right|_{X_{i}}=\left.g\right|_{X_{i}} \mu_{i^{-}}$-qs para todo $i \in I \backslash I^{\prime}$.

OBSERVAÇÃo 4.2.2. Se $\left(X_{i}\right)_{i \in I}$ é uma decomposição essencial para um espaço de medida completa $(X, \mathcal{A}, \mu)$, e para cada $i \in I, \mathcal{A}_{i}=\left.\mathcal{A}\right|_{X_{i}}$ e $\mu_{i}=\left.\mu\right|_{\mathcal{A}_{i}}$, então $\mu_{i}$ é completa e $0<\mu_{i}\left(X_{i}\right)<+\infty$ para todo $i \in I$. De fato, sejam $i \in I$ fixado, $A \in \mathcal{A}_{i} \operatorname{com} \mu_{i}(A)=0$ e $B$ um subconjunto arbitrário de $A$. Se $A \in \mathcal{A}_{i}$ então $A \subset X_{i}, A \in \mathcal{A}$ e $\mu(A)=\mu_{i}(A)=0$. Como $\mu$ é uma medida completa, temos que $B \in \mathcal{A}$ e conseqüentemente $B \in \mathcal{A}_{i}$ (pois $B \subset A)$; portanto $\mu_{i}$ é completa. Além disso, uma vez que $\left(X_{i}\right)_{i \in I}$ é uma decomposição essencial para $(X, \mathcal{A}, \mu)$, temos $0<\mu\left(X_{i}\right)=\mu_{i}\left(X_{i}\right)<+\infty$.

Proposição 4.2.3. Sejam $(X, \mathcal{A}, \mu)$ um espaço de medida perfeita e $\left(X_{i}\right)_{i \in I}$ uma decomposição essencial para $X$. Para cada $i \in I$, denotamos por $\mathcal{A}_{i}$ a $\sigma$-álgebra de todos os elementos de $\mathcal{A}$ contidos em $X_{i}$ e por $\mu_{i}$ a medida em $\mathcal{A}_{i}$ obtida pela restrição de $\mu$ (i.e., $\mathcal{A}_{i}=\left.\mathcal{A}\right|_{X_{i}}$ e $\mu_{i}=\left.\mu\right|_{\mathcal{A}_{i}}$ ), e assim $\left(X_{i}, \mathcal{A}_{i}, \mu_{i}\right)$ é um espaço de medida completa com $0<\mu_{i}\left(X_{i}\right)<+\infty$; considere a soma externa $\sum_{i \in I}\left(X_{i}, \mathcal{A}_{i}, \mu_{i}\right)$. Seja $\phi: \sum_{i \in I} X_{i} \rightarrow X$ a aplicação canônica cuja restrição a cada $X_{i}$ é igual à aplicação inclusão $X_{i} \rightarrow X$. Então $\phi$ é uma aplicação quociente e a relação de equivalência $\sim$ induzida em $\sum_{i \in I} X_{i}$ por $\phi$ satisfaz a propriedade (*) do enunciado da Proposição 4.2.1.

Demonstração. Seja $\left(X^{\prime}, \mathcal{A}^{\prime}, \mu^{\prime}\right)=\sum_{i \in I}\left(X_{i}, \mathcal{A}_{i}, \mu_{i}\right)$. Vamos mostrar que $\phi$ é uma aplicação quociente, ou seja, que $\phi_{*} \mathcal{A}^{\prime}=\mathcal{A}$ e $\phi_{*} \mu^{\prime}=\mu$. Se $\left(X_{i}\right)_{i \in I}$ é uma decomposição essencial para $X$ e $\mu$ é uma medida perfeita então, pela Proposição 4.1.7, $A \subset X$ é mensurável se e somente se $A \cap X_{i}$ é mensurável para todo $i \in I$, e para cada $A \in \mathcal{A}$,

$$
\mu(A)=\mu_{\mathrm{lb}}(A)=\sum_{i \in I} \mu\left(A \cap X_{i}\right) .
$$

Assim, $\phi_{*} \mathcal{A}^{\prime}=\left\{A \subset X: \phi^{-1}(A) \in \mathcal{A}^{\prime}\right\}$

$$
\begin{aligned}
& =\left\{A \subset X: \phi^{-1}(A) \cap X_{i} \in \mathcal{A}_{i} \text { para todo } i \in I\right\} \\
& =\left\{A \subset X: A \cap X_{i} \in \mathcal{A}_{i} \text { para todo } i \in I\right\}=\mathcal{A} ;
\end{aligned}
$$

e $\mu(A)=\sum_{i \in I} \mu\left(A \cap X_{i}\right)=\sum_{i \in I} \mu_{i}\left(\phi^{-1}(A) \cap X_{i}\right)=\mu^{\prime}\left(\phi^{-1}(A)\right)=\left(\phi_{*} \mu^{\prime}\right)(A)$, 
para cada $A \in \mathcal{A}$, i.e., $\phi_{*} \mu^{\prime}=\mu$. Mostraremos agora que a relação $\sim$ induzida em $X^{\prime}=\sum_{i \in I} X_{i}$ por $\phi$ satisfaz a propriedade $(*)$ do enunciado da Proposição 4.2.1. Lembramos que se $x, y \in X^{\prime}$ então $x \sim y$ se e somente se $\phi(x)=\phi(y)$. Para cada $i \in I$ e para todo $x, y \in X_{i}$, temos $x \sim y$ se e somente $\phi(x)=\phi(y)$, i.e., se e somente se $x=y$. Por outro lado, para todo $i, j \in I$ com $i \neq j$, temos:

$$
\begin{aligned}
\mathcal{B}_{j} & =\left\{x \in X_{i}: x \sim y \text { para algum } y \in X_{j}\right\} \\
& =\left\{x \in X_{i}: \phi(x)=\phi(y) \text { para algum } y \in X_{j}\right\} \\
& =\left\{x \in X_{i}: x=y \text { para algum } y \in X_{j}\right\} \\
& =\left\{x \in X_{i}: x \in X_{j}\right\} \\
& =X_{i} \cap X_{j} .
\end{aligned}
$$

Como $\left(X_{i}\right)_{i \in I}$ é uma decomposição essencial para $X$, segue que o conjunto $\mathcal{B}_{j}$ tem medida nula. Portanto a relação $\sim$ verifica a propriedade $(*)$.

Corolário 4.2.4. Sob as condições e notações do enunciado da Proposição 4.2.3, suponhamos que o conjunto quociente $\left(\sum_{i \in I} X_{i}\right) / \sim$ esteja munido com a $\sigma$-álgebra e medida co-induzidas pela aplicação canônica

$$
\mathfrak{q}: \sum_{i \in I} X_{i} \longrightarrow\left(\sum_{i \in I} X_{i}\right) / \sim
$$

Então a aplicação $\phi$ induz um isomorfismo $\bar{\phi}:\left(\sum_{i \in I} X_{i}\right) / \sim \rightarrow \phi\left(\sum_{i \in I} X_{i}\right)$ tal que $\phi\left(\sum_{i \in I} X_{i}\right)$ é um subconjunto mensurável de $X$ cujo complemento tem medida nula e é comutativo o diagrama

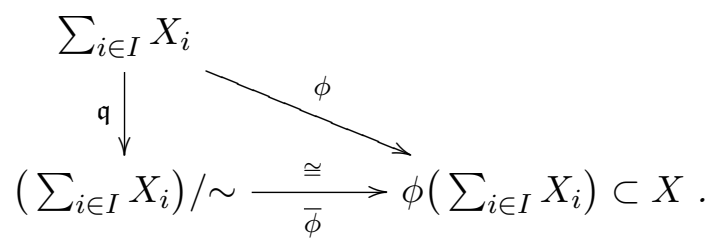

DemonstraÇÃo. Segue imediatamente do Lema 1.4.88.

Corolário 4.2.5. Sob as condições e notações do enunciado da Proposição 4.2.3, para todo $p \in[1,+\infty[$ a aplicação

$$
L^{p}(X, \mathcal{A}, \mu) \ni f \longmapsto f \circ \phi \in L^{p}\left(\sum_{i \in I}\left(X_{i}, \mathcal{A}_{i}, \mu_{i}\right)\right),
$$

induzida por $\phi$ em $L^{p}$, é uma isometria linear. 
Demonstração. Consideremos as aplicações q e $\bar{\phi}$ como no Corolário 4.2.4. Seja $\left(X^{\prime}, \mathcal{A}^{\prime}, \mu^{\prime}\right)=\sum_{i \in I}\left(X_{i}, \mathcal{A}_{i}, \mu_{i}\right)$. Então:

$\left(\sum_{i \in I} X_{i}, \mathcal{A}^{\prime}, \mu^{\prime}\right) \stackrel{\mathfrak{q}}{\longrightarrow}\left(\left(\sum_{i \in I} X_{i}\right) / \sim, \mathfrak{q}_{*} \mathcal{A}^{\prime}, \mathfrak{q}_{*} \mu^{\prime}\right) \stackrel{\bar{\phi}}{\longrightarrow}\left(\phi\left(\sum_{i \in I} X_{i}\right), \mathcal{A}^{\prime \prime}, \mu^{\prime \prime}\right)$,

em que $\mathcal{A}^{\prime \prime}=\left.\mathcal{A}\right|_{\phi\left(\sum_{i \in I} X_{i}\right)}$ e $\mu^{\prime \prime}=\left.\mu\right|_{\mathcal{A}^{\prime \prime}}$. Seja $p \in[1,+\infty[$. Sendo $\bar{\phi}$ um isomorfismo temos, pelo Corolário 1.4.87, que a aplicação induzida por $\bar{\phi}$ em $L^{p}$

$$
L^{p}\left(\phi\left(\sum_{i \in I} X_{i}\right)\right) \ni f \longmapsto f \circ \bar{\phi} \in L^{p}\left(\left(\sum_{i \in I} X_{i}\right) / \sim\right),
$$

é uma isometria linear. Por outro lado, pela Proposição 4.2.1, a aplicação induzida por $\mathfrak{q}$ em $L^{p}$,

$$
L^{p}\left(\left(\sum_{i \in I} X_{i}\right) / \sim\right) \ni f \longmapsto f \circ \mathfrak{q} \in L^{p}\left(\sum_{i \in I}\left(X_{i}, \mathcal{A}_{i}, \mu_{i}\right)\right),
$$

também é uma isometria linear. Uma vez que o complemento de $\phi\left(\sum_{i \in I} X_{i}\right)$ em $X$ tem medida nula, segue pela Observação 1.4 .82 que, a menos de identificação isométrica, $L^{p}\left(\phi\left(\sum_{i \in I} X_{i}\right), \mathcal{A}^{\prime \prime}, \mu^{\prime \prime}\right)=L^{p}(X, \mathcal{A}, \mu)$. Assim, para cada $f \in L^{p}(X, \mathcal{A}, \mu)$ temos que $f \circ \phi=f \circ(\bar{\phi} \circ \mathfrak{q})=(f \circ \bar{\phi}) \circ \mathfrak{q}$ está em $L^{p}\left(\phi\left(\sum_{i \in I} X_{i}\right), \mathcal{A}^{\prime \prime}, \mu^{\prime \prime}\right)$, o que mostra que a aplicação induzida por $\phi$ em $L^{p}$ é uma isometria linear.

A composição da aplicação (4.2.1) com a isometria (3.2.3) é dada por:

$$
L^{p}(X, \mathcal{A}, \mu) \ni f \longmapsto\left(\left.f\right|_{X_{i}}\right)_{i \in I} \in \bar{\bigoplus}_{i \in I}^{p} L^{p}\left(X_{i}, \mathcal{A}_{i}, \mu_{i}\right) .
$$

Com efeito,

$$
\begin{gathered}
L^{p}(X, \mathcal{A}, \mu) \ni f \stackrel{(4.2 .1)}{\longmapsto} f \circ \phi \in L^{p}\left(\sum_{i \in I}\left(X_{i}, \mathcal{A}_{i}, \mu_{i}\right)\right), \\
L^{p}\left(\sum_{i \in I}\left(X_{i}, \mathcal{A}_{i}, \mu_{i}\right)\right) \ni f \circ \phi \stackrel{(3.2 .3)}{\longmapsto}\left(\left.(f \circ \phi)\right|_{X_{i}}\right)_{i \in I} \in \bar{\bigoplus}_{i \in I}^{p} L^{p}\left(X_{i}, \mathcal{A}_{i}, \mu_{i}\right),
\end{gathered}
$$

e $\left(\left.(f \circ \phi)\right|_{X_{i}}\right)_{i \in I}=\left(\left.f\right|_{X_{i}}\right)_{i \in I}$, uma vez que $\left.\phi\right|_{X_{i}}: X_{i} \rightarrow X$ é a inclusão.

Para $p<+\infty$, a aplicação (4.2.2) é uma isometria linear e para $p=+\infty$, (4.2.2) é, em geral, apenas uma imersão isométrica. Da comutatividade dos diagramas (3.2.5) e (1.4.6) temos, para todo $p \in[1,+\infty[, q \in] 1,+\infty]$ com 
$\frac{1}{p}+\frac{1}{q}=1$, um novo diagrama comutativo:

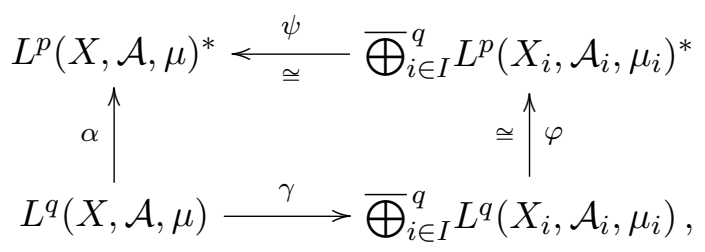

no qual $\psi$ é a composição da transposta da aplicação (4.2.1) com a aplicação (3.2.4) , $\gamma$ é a versão da aplicação (4.2.2) para $L^{q}, \alpha$ é a $(q, p)$-aplicação de Riesz (1.4.2) para o espaço $(X, \mathcal{A}, \mu)$ e $\varphi$ é dada pela $(q, p)$-aplicação de Riesz (1.4.2) para o espaço $\left(X_{i}, \mathcal{A}_{i}, \mu_{i}\right)$ em cada coordenada. Com efeito, considerando os espaços de medida $\sum_{i \in I}\left(X_{i} \mathcal{A}_{i}, \mu_{i}\right)$ e $(X, \mathcal{A}, \mu)$, e a aplicação quociente $\phi: \sum_{i \in I} X_{i} \rightarrow X$, a combinação dos diagramas (3.2.5) e (1.4.6) resulta no seguinte diagrama:

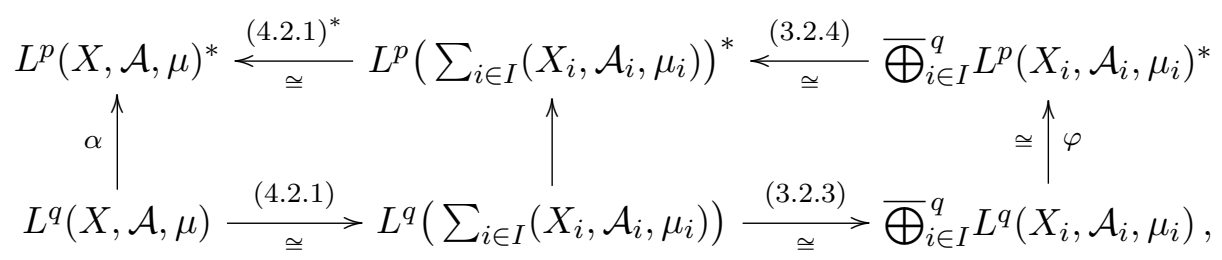

Observe ainda que, como $\mu_{i}\left(X_{i}\right)<+\infty$ para todo $i \in I$, a aplicação $\varphi$ do diagrama (4.2.3) é uma isometria, pelo Teorema de Representação de Riesz para espaços de medida finita. De fato, dada $g \in L^{q}(X, \mathcal{A}, \mu)$, aplicando (4.2.2) obtemos $\left(\left.g\right|_{X_{i}}\right)_{i \in I} \in \bar{\bigoplus}_{i \in I}^{q} L^{q}\left(X_{i}, \mathcal{A}_{i}, \mu_{i}\right)$. Para cada $i \in I$, a $(q, p)$-aplicação de Riesz (1.4.2) associa $g \in L^{q}\left(X_{i}, \mathcal{A}_{i}, \mu_{i}\right)$ ao funcional $\alpha_{i} \in L^{p}\left(X_{i}, \mathcal{A}_{i}, \mu_{i}\right)^{*}$ tal que $\left\|\alpha_{i}\right\|=\left\|\left.g\right|_{X_{i}}\right\|_{q}$. Então

$$
\begin{gathered}
\left\|\left(\alpha_{i}\right)_{i \in I}\right\|_{q}=\left(\sum_{i \in I}\left\|\alpha_{i}\right\|^{q}\right)^{\frac{1}{q}}=\left(\sum_{i \in I}\left\|\left.g\right|_{X_{i}}\right\|_{q}^{q}\right)^{\frac{1}{q}}=\left\|\left(\left.g\right|_{X_{i}}\right)_{i \in I}\right\|_{q}, \text { se } q<+\infty \\
\text { e }\left\|\left(\alpha_{i}\right)_{i \in I}\right\|_{\infty}=\sup _{i \in I}\left\|\alpha_{i}\right\|=\sup _{i \in I}\left\|\left.g\right|_{X_{i}}\right\|=\left\|\left(\left.g\right|_{X_{i}}\right)_{i \in I}\right\|_{\infty} .
\end{gathered}
$$

Por outro lado, dada $\left(\alpha_{i}\right)_{i \in I}$ em $\bar{\bigoplus}_{i \in I}^{q} L^{q}\left(X_{i}, \mathcal{A}_{i}, \mu_{i}\right)$, para cada $i \in I$ existe $g_{i} \in L^{q}\left(X_{i}, \mathcal{A}_{i}, \mu_{i}\right)$ tal que $\alpha_{i}$ é a imagem de $g_{i}$ pela aplicação de Riesz. Assim, a família $\left(g_{i}\right)_{i \in I} \in \prod_{i \in I} L^{q}\left(X_{i}, \mathcal{A}_{i}, \mu_{i}\right)$ é tal que

$$
\left\|\left(\left.g\right|_{X_{i}}\right)_{i \in I}\right\|_{q}=\left\|\left(\alpha_{i}\right)_{i \in I}\right\|_{q}<+\infty,
$$

e portanto $\left(g_{i}\right)_{i \in I} \in \bar{\bigoplus}_{i \in I}^{q} L^{q}\left(X_{i}, \mathcal{A}_{i}, \mu_{i}\right)$. Claramente $\varphi\left(\left(\left.g\right|_{X_{i}}\right)_{i \in I}\right)=\left(\alpha_{i}\right)_{i \in I}$, o que mostra que $\varphi$ é sobrejetora. 
O diagrama (4.2.4) a seguir, no qual $X^{\prime}=\sum_{i \in I} X_{i}$, é o diagrama (4.2.3) expandido, e será útil para uma melhor compreensão da demonstração do Teorema 4.2.7.

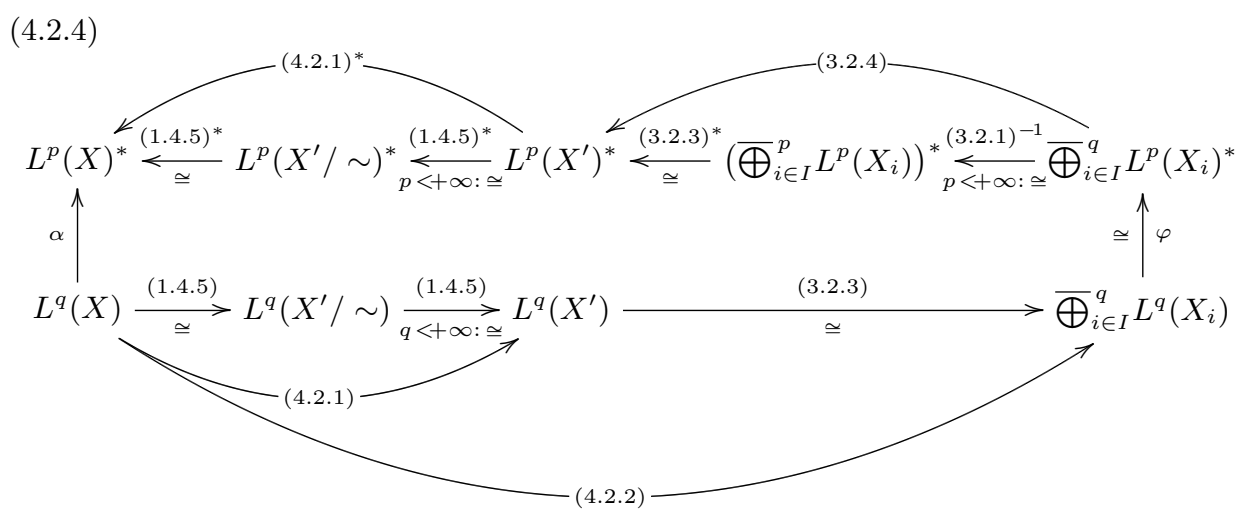

Teorema 4.2.6. Seja $(X, \mathcal{A}, \mu)$ um espaço de medida arbitrário. Se $p, q \in] 1,+\infty\left[e^{\frac{1}{p}}+\frac{1}{q}=1\right.$ então a $(q, p)$-aplicação de Riesz (1.4.2) para $(X, \mathcal{A}, \mu)$ é uma isometria linear.

Demonstração. Pela Proposição 2.3.6 e pela comutatividade do diagrama (2.3.2) podemos substituir $\mu$ por sua versão perfeita $\mu_{\mathfrak{p}}$. Portanto, podemos assumir que $\mu$ é uma medida perteita. Uma vez que $p, q \in] 1,+\infty[$, o Corolário 4.2.5 implica que as aplicações $\psi$ e $\gamma$ do diagrama (4.2.3) são isometrias lineares. Além disso, como já vimos acima, a aplicação $\varphi$ desse mesmo diagrama também é uma isometria linear. Da comutatividade do diagrama (4.2.3) segue que a $(q, p)$-aplicação de Riesz (1.4.2) para o espaço $(X, \mathcal{A}, \mu)$ é uma isometria linear.

TeOrema 4.2.7. Se um espaço de medida perfeita $(X, \mathcal{A}, \mu)$ admite uma decomposição $\left(X_{i}\right)_{i \in I}$ então a sua aplicação de Riesz (1.4.4) é uma isometria linear.

Demonstração. Se $\left(X_{i}\right)_{i \in I}$ é uma decomposição para $(X, \mathcal{A}, \mu)$ então a aplicação $\phi: \sum_{i \in I} X_{i} \rightarrow X$ que aparece no enunciado da Proposição 4.2.3 é injetora, pois sua restrição a cada $X_{i}$ é a inclusão e os conjuntos $X_{i}$ são dois a dois disjuntos. Portanto, a aplicação $\mathfrak{q}: \sum_{i \in I} X_{i} \longrightarrow\left(\sum_{i \in I} X_{i}\right) / \sim$, do enunciado do Corolário 4.2.4, é simplesmente a função identidade de $\sum_{i \in I} X_{i}$. Assim, com a notação desse corolário, $\phi=\bar{\phi} \circ \mathfrak{q}=\bar{\phi}$, e portanto $\phi$ 
é um isomorfismo de $\sum_{i \in I} X_{i}$ sobre o conjunto mensurável $\phi\left(\sum_{i \in I} X_{i}\right)$, cujo complemento em $X$ tem medida nula. Isso prova que a aplicação induzida por $\phi$ em $L^{\infty}$,

$$
L^{\infty}\left(\phi\left(\sum_{i \in I} X_{i}\right)\right)=L^{\infty}(X, \mathcal{A}, \mu) \ni f \longmapsto f \circ \phi \in L^{\infty}\left(\sum_{i \in I}\left(X_{i}, \mathcal{A}_{i}, \mu_{i}\right)\right),
$$

é uma isometria linear, e portanto as aplicações $\psi$ e $\gamma$ do diagrama (4.2.3) são isometrias lineares para $p=1$ e $q=+\infty$. Assim, da comutatividade do diagrama (4.2.3) concluímos que a aplicação de Riesz (1.4.4) para o espaço $(X, \mathcal{A}, \mu)$ é uma isometria linear.

Corolário 4.2.8. Seja $(X, \mathcal{A}, \mu)$ um espaço de medida perfeita. Se $\operatorname{dim}(X, \mathcal{A}, \mu) \leq \aleph_{1}$ então a aplicação de Riesz (1.4.4) de $(X, \mathcal{A}, \mu)$ é uma isometria linear.

Demonstração. Segue da Proposição 4.1.16 e do Teorema 4.2.7

Corolário 4.2.9. A aplicação de Riesz (1.4.4) do espaço de medida $(X, \mathcal{A}, \mu)$ contruído no Exemplo 2.4.1 é uma isometria linear se e somente se $\left|C_{1}\right|=\left|C_{2}\right|=\aleph_{1}$.

DemonstraÇÃo. Segue imediatamente dos Corolários 2.4.5 e 4.2.8.

O diagrama comutativo (4.2.3) permite-nos ver qual é exatamente o obstáculo para a bijetividade da aplicação de Riesz (1.4.4) de um espaço de medida perfeita $(X, \mathcal{A}, \mu)$. A saber, a aplicação de Riesz (1.4.4) do espaço $(X, \mathcal{A}, \mu)$ é uma isometria linear se e somente se é sobrejetora a aplicação $\gamma$ do diagrama (4.2.3).

Lema 4.2.10. Sejam $(X, \mathcal{A}, \mu)$ um espaço de medida perfeita e $\left(X_{i}\right)_{i \in I}$ uma decomposição essencial para $X$. Para $q=+\infty$, a imagem da aplicação $\gamma$ do diagrama (4.2.3) consiste das famílias $\left(f_{i}\right)_{i \in I} \in \bar{\bigoplus}_{i \in I}^{\infty} L^{\infty}\left(X_{i}, \mathcal{A}_{i}, \mu_{i}\right)$ para as quais existe uma aplicação $f: X \rightarrow \mathbb{R}$ tal que $\left.f\right|_{X_{i}}=f_{i} \mu_{i}$-qs, para todo $i \in I$. Em particular, a aplicação de Riesz (1.4.4) de $(X, \mathcal{A}, \mu)$ é uma isometria linear se e somente se vale a seguinte condição: dada uma família $\left(f_{i}\right)_{i \in I} \in \prod_{i \in I} L^{\infty}\left(X_{i}, \mathcal{A}_{i}, \mu_{i}\right)$ com $\sup _{i \in I}\left\|f_{i}\right\|_{\infty}<+\infty$ então existe uma aplicação $f: X \rightarrow \mathbb{R}$ com $\left.f\right|_{X_{i}}=f_{i} \mu_{i}$-qs, para todo $i \in I$.

Demonstração. Lembramos que a aplicação $\gamma$ do diagrama (4.2.3) é a composição das aplicações (3.2.3) e (4.2.1), o que pode ser visto abaixo na 
sua versão para $p=+\infty$ :

$$
\begin{gathered}
L^{\infty}(X) \ni f \stackrel{(4.2 .1)}{\longmapsto} f \circ \phi \in L^{\infty}\left(\sum_{i \in I} X_{i}\right) \text { e } \\
L^{\infty}\left(\sum_{i \in I} X_{i}\right) \ni f \circ \phi \stackrel{(3.2 .3)}{\longmapsto}\left(\left.(f \circ \phi)\right|_{X_{i}}\right)_{i \in I}=\left(\left.f\right|_{X_{i}}\right)_{i \in I} \in \bar{\bigoplus}_{i \in I}^{\infty} L^{\infty}\left(X_{i}\right),
\end{gathered}
$$

na qual $\phi$ é aplicação que aparece no enunciado da Proposição 4.2.3. Pelo Lema 1.4.86, a imagem da aplicação (4.2.1) é o conjunto

$$
\left\{g \in L^{\infty}\left(\sum_{i \in I}\left(X_{i}, \mathcal{A}_{i}, \mu_{i}\right)\right) \text { : existe } f: X \rightarrow \mathbb{R} \text { tal que } f \circ \phi=g \mu \text {-qs }\right\} .
$$

Seja $\mathfrak{B}=\bar{\bigoplus}_{i \in I}^{\infty} L^{\infty}\left(X_{i}, \mathcal{A}_{i}, \mu_{i}\right)$. Uma vez que a aplicação (3.2.3) é uma isometria, temos:

$$
\begin{aligned}
\operatorname{Im}(\gamma) & =\left\{\left(\left.g\right|_{X_{i}}\right)_{i \in I} \in \mathfrak{B}: \text { existe } f: X \rightarrow \mathbb{R} \text { tal que } f \circ \phi=g \mu \text {-qs }\right\} \\
& =\left\{\left(\left.g\right|_{X_{i}}\right)_{i \in I} \in \mathfrak{B}: \text { existe } f: X \rightarrow \mathbb{R} \text { tal que }\left.f \circ \phi\right|_{X_{i}}=\left.g\right|_{X_{i}} \mu_{i^{-}} \text {qs }\right\} .
\end{aligned}
$$

Como $\phi$ restrita a cada $X_{i}$ é a aplicação inclusão segue que

$$
\operatorname{Im}(\gamma)=\left\{\left(f_{i}\right)_{i \in I} \in \mathfrak{B}: \text { existe } f: X \rightarrow \mathbb{R} \text { tal que }\left.f\right|_{X_{i}}=f_{i} \mu_{i} \text {-qs }\right\} .
$$

Assim, a aplicação de Riesz (1.4.4) para o espaço $(X, \mathcal{A}, \mu)$ é uma isometria se e somente $\operatorname{se} \operatorname{Im}(\gamma)=\mathfrak{B}$, ou seja, se e somente se $\mathfrak{B} \subset \operatorname{Im}(\gamma)$. Em outras palavras, se e somente se para cada família $\left(f_{i}\right)_{i \in I} \in \prod_{i \in I} L^{\infty}\left(X_{i}, \mathcal{A}_{i}, \mu_{i}\right)$ com $\sup _{i \in I}\left\|f_{i}\right\|_{\infty}<+\infty$ existe uma função $f: X \rightarrow \mathbb{R}$ com $\left.f\right|_{X_{i}}=f_{i} \mu_{i}$-qs, para todo $i \in I$.

Teorema 4.2.11. Seja $(X, \mathcal{A}, \mu)$ um espaço de medida perfeita com

$$
\operatorname{dim}(X, \mathcal{A}, \mu) \leq 2^{\aleph_{0}}
$$

Então a aplicação de Riesz (1.4.4) de $(X, \mathcal{A}, \mu)$ é uma isometria linear se e somente se $X$ admite uma decomposição.

Demonstração. Se $X$ admite uma decomposição então sua aplicação de Riesz (1.4.4) é uma isometria linear, pelo Teorema 4.2.7. Reciprocamente, suponhamos que a aplicação de Riesz (1.4.4) de $X$ seja uma isometria linear. Seja $\left(X_{i}\right)_{i \in I}$ uma decomposição essencial para $X$. Uma vez que a cardinalidade de $I$ é menor ou igual a $2^{\aleph_{0}}$, podemos encontrar uma aplicação injetora $c: I \rightarrow[0,1]$. Para cada $i \in I$, seja $f_{i} \in L^{\infty}\left(X_{i}, \mathcal{A}_{i}, \mu_{i}\right)$ a aplicação constante $f_{i}(x)=c(i)$, para todo $x \in X_{i}$. Assim, $\left(f_{i}\right)_{i \in I}$ é uma família em $\prod_{i \in I} L^{\infty}\left(X_{i}, \mathcal{A}_{i}, \mu_{i}\right)$ com $\sup _{i \in I}\left\|f_{i}\right\|_{\infty}=\sup _{i \in I} c(i)=1$, e portanto, 
pelo Lema 4.2.10, existe uma função $f: X \rightarrow \mathbb{R}$ tal que $\left.f\right|_{X_{i}}=c(i) \mu_{i}$-qs, para todo $i \in I$. Seja $Y_{i}=X_{i} \cap f^{-1}(c(i))$. Como $\left.f\right|_{X_{i}}=c(i) \mu_{i}$-qs temos $\mu\left(X_{i} \backslash Y_{i}\right)=0$ para todo $i \in I$. Portanto $\left(Y_{i}\right)_{i \in I}$ é um refinamento de $\left(X_{i}\right)_{i \in I}$. Além disso, para $i, j \in I, i \neq j$, temos $c(i) \neq c(j)$, o que implica $Y_{i} \cap Y_{j}=\emptyset$. Portanto $\left(Y_{i}\right)_{i \in I}$ é uma decomposição para $(X, \mathcal{A}, \mu)$.

O teorema 4.2.11 nos diz que para espaços de medida perfeita com dimensão menor ou igual à cardinalidade do continuum, admitir decomposição é condição necessária e suficiente para que a aplicação de Riesz (1.4.4) desse espaço seja uma isometria linear. Uma questão natural é o que ocorre se a dimensão do espaço de medida (perfeita) for maior do que $2^{\aleph_{0}}$. Em $[\mathbf{1 0}]$, Fremlin [Exemplo 11, pág. 165] mostra que é consistente com ZFC a existência de um exemplo de um espaço de medida perfeita ${ }^{1}$ para o qual sua aplicação de Riesz (1.4.4) é uma isometria², mas não há decomposição.

${ }^{1}$ c.l.d. na terminologia do autor.

${ }^{2} \mathrm{Na}$ verdade, o autor apresenta um exemplo de um espaço de Maharan (localizável) que, pelo teorema de Segal $([\mathbf{2 0}],[\mathbf{2 3}])$ é um espaço de medida cuja aplicação de Riesz (1.4.4) é uma isometria linear. 


\section{Referências Bibliográficas}

[1] C. D. Aliprantis, K. C. Border, Infinite Dimensional Analysis, Springer-Verlag, 2006.

[2] C. D. Aliprantis, O. Burkinshaw, Principles of Real Analysis, Academic Press, 1998.

[3] R. G. Bartle, The Elements of Integration and Lebesgue Measure, John Wiley \& Sons, Inc., 1995.

[4] F. U. Coelho, M. L. Lourenço, Um Curso de Álgebra Linear, Editora da Universidade de São Paulo, 2001.

[5] Y. Eidelman, V. Milman, A. Tsolomitis, Functional Analysis - An Introduction, Graduate Studies in Mathematics v.66, AMS, 2004.

[6] H. B. Enderton, Elements of Set Theory, Academic Press, 1977.

[7] R. Engelking, General Topology, Sigma Series in Pure Mathematics v.6, Heldermann Verlag, 1989.

[8] P. J. Fernandez, Medida e Integração, Instituto de Matemática Pura e Aplicada (projeto Euclides), 1976.

[9] G. B. Folland, Real Analysis: Modern Techniques and Their Application, John Wiley \& Sons, Inc., 1999.

[10] D. H. Fremlin, Decomposable Measure Spaces, Z. Wahrscheinlichkeitstheorie verw. Gebiete 45, 159-167 (1978).

[11] P. R. Halmos, Measure Theory, Springer-Verlag, 1974.

[12] P. R. Halmos, Naive Set Theory, Litton Educational Publishing,Inc., 1960.

[13] K. Hoffman, R. Kunze Linear Algebra, Prentice-Hall,Inc., 1971.

[14] C. S. Hönig, Análise Funcional e Aplicações, vol.1, Publicações do Instituto de Matemática e Estatística da USP, 1970.

[15] T. Jech, Set Theory, 3rd Millennium ed., rev. and expanded, Springer, 2006.

[16] T. Jech, K. Hrbacek, Introduction to Set Theory, Marcel Dekker,Inc., 1999.

[17] E. Kreyszig, Introductory Functional Analysis with applications, John Wiley \& Sons,Inc., 1978.

[18] E. L. Lima, Espaços Métricos, Instituto de Matemática Pura e Aplicada (projeto Euclides), 1981.

[19] E. J. McShane, Families of Measures and Representations of Algebras of Operators, Transactions of the AMS, vol. 102, 328-345 (1962).

[20] M. M. Rao, Measure Theory and Integration, John Wiley \& Sons, Inc., 1987.

[21] H. L. Royden, Real Analysis, Macmillan Publishing Company, 1963.

[22] H. L. Royden, Real Analysis, Third edition, Macmillan Publishing Company, 1968. 
[23] I. E. Segal, Equivalence of measure spaces, American Journal of Mathematics, vol. 73, 275-313 (1951).

[24] E. M. Stein, R. Shakarchi, Real Analysis: Measure Theory, Integration, \& Hilbert Spaces, Princeton Lectures in Analysis III, Princeton University Press, 2005.

[25] A. C. Zaanen, Integration, North-Holland Publishing Company - John Wiley \& Sons, Inc., 1967. 